Nevada

Environmental

Restoration

Project

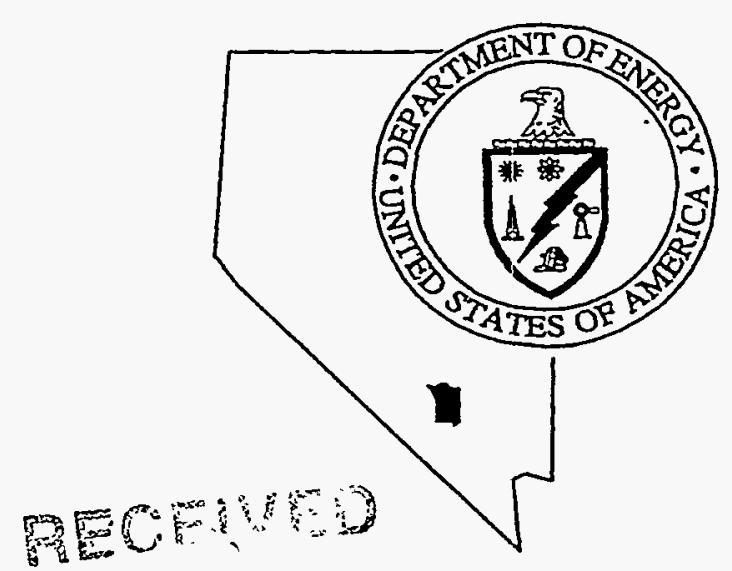

APR O 1999

OSTI

\title{
Closure Report
}

\section{Nevada Test Site Underground Storage Tank Number 12-13-1: Nevada Division of Emergency Management Number H931130E Corrective Action Unit 450}

January 1997

Environmental Restoration Division

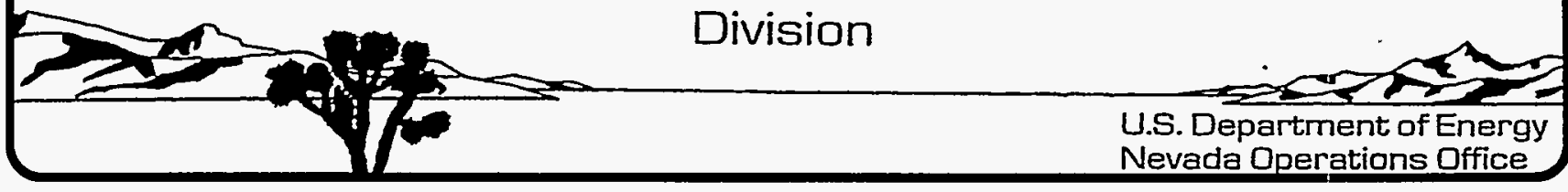


DOE/NV/11718-060

UC-902

\section{CLOSURE REPORT}

NEVADA TEST SITE UNDERGROUND STORAGE

TANK NUMBER 12-13-1:

NEVADA DIVISION OF EMERGENCY MANAGEMENT

CASE NUMBER H931130E

CORRECTIVE ACTION UNIT 450

January 1997

Prepared for

U.S. Department of Energy

Environmental Restoration Division

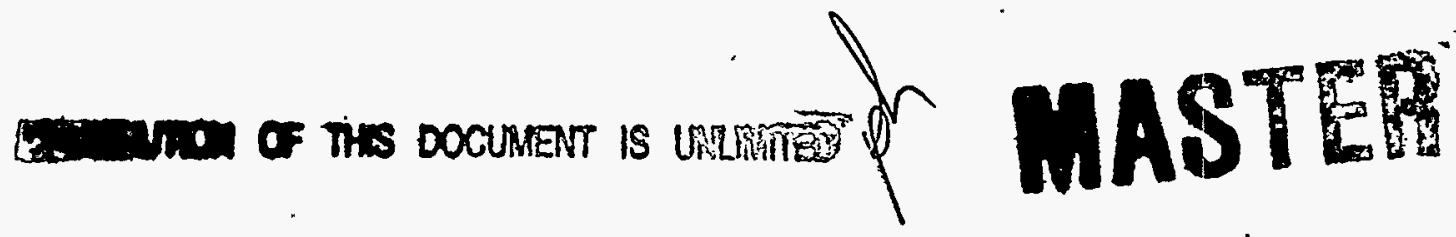

Prepared by

Bechtel Nevada

Remediation Projects 
This report has been reproduced from the best available copy.

DOE and DOE contractors can obtain copies of this report from the Office of Scientific and Technical Information, P.O. Box 62, Oak Ridge, TN 37831, (615) 576-8401.

This report is publicly available from the National Technical Information Service, U.S. Department of Commerce, 5285 Port Royal Road, Springfield, VA 22161, (703) 487-4650. 


\section{DISCLAIMERR}

Portions of this document may be illegible in electronic image products. Images are produced from the best available original document. 


\section{DISCLAIMER}

This report was prepared as an account of work sponsored by an agency of the United States Government. Neither the United States Government nor any agency thereof, nor any of their employees, make any warranty, express or implied, or assumes any legal liability or responsibility for the accuracy, completeness, or usefulness of any information, apparatus, product, or process disclosed, or represents that its use would not infringe privately owned rights. Reference herein to any specific commercial product, process, or service by trade name, trademark, manufacturer, or otherwise does not necessarily constitute or imply its endorsement, recommendation, or favoring by the United States Government or any agency thereof. The views and opinions of authors expressed herein do not necessar. ily state or reflect those of the United States Government or any agency thereof. 


\section{TABLE OF CONTENTS}

SECTION

$\underline{P A G E}$

Figures $\ldots \ldots \ldots \ldots \ldots \ldots \ldots \ldots \ldots \ldots \ldots \ldots \ldots \ldots \ldots$ ii

Table ..........................

Appendices $\ldots \ldots \ldots \ldots \ldots \ldots \ldots \ldots \ldots \ldots \ldots \ldots \ldots \ldots$ ii

Abbreviations and Acronyms $\ldots \ldots \ldots \ldots \ldots \ldots \ldots \ldots \ldots \ldots \ldots \ldots \ldots \ldots \ldots \ldots$ iii

1.0 INTRODUCTION $\ldots \ldots \ldots \ldots \ldots \ldots \ldots \ldots \ldots \ldots \ldots \ldots \ldots \ldots \ldots \ldots \ldots$

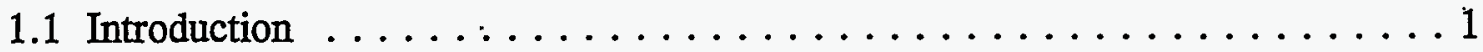

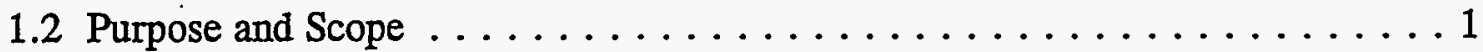

1.3 Site Description . . . . . . . . . . . . . . . . . 2

2.0 TANK CLOSURE ACTIVITIES $\ldots \ldots \ldots \ldots \ldots \ldots \ldots \ldots \ldots \ldots \ldots \ldots \ldots$

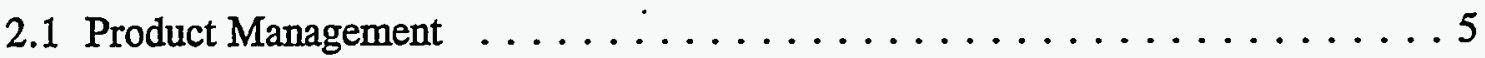

2.1.1 Tank Sampling and Results $\ldots \ldots \ldots \ldots \ldots \ldots \ldots \ldots$

2.1.2 Product Removal and Disposal . . . . . . . . . . . . 6

2.2 Soil Excavation and Tank Removal $\ldots \ldots \ldots \ldots \ldots \ldots \ldots \ldots$

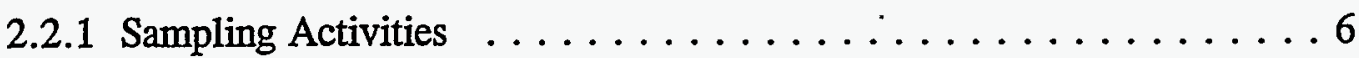

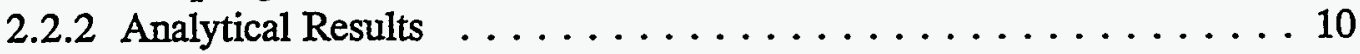

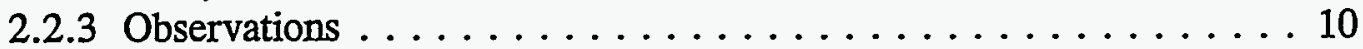

2.2 .4 Backfill ........................ 10

2.3 Spill Notification Report $\ldots \ldots \ldots \ldots \ldots \ldots \ldots \ldots \ldots \ldots \ldots \ldots$

3.0 REMEDIAL ACTIVITIES $\ldots \ldots \ldots \ldots \ldots \ldots \ldots \ldots \ldots \ldots \ldots \ldots \ldots \ldots$

3.1 Excavation and Sampling Activities $\ldots \ldots \ldots \ldots \ldots \ldots \ldots \ldots \ldots \ldots$

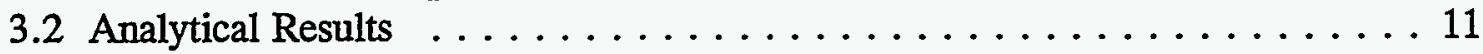

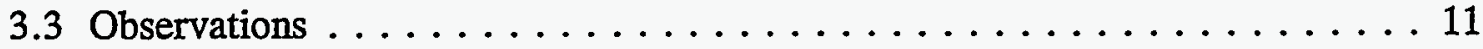

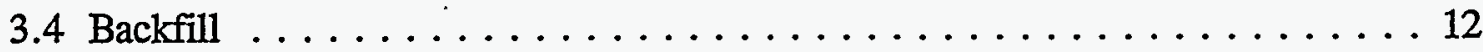

4.0 RESIDUALS MANAGEMENT $\ldots \ldots \ldots \ldots \ldots \ldots \ldots \ldots \ldots \ldots \ldots \ldots$

4.1 Soil Disposal . . . . . . . . . . . . . . . . . . 13

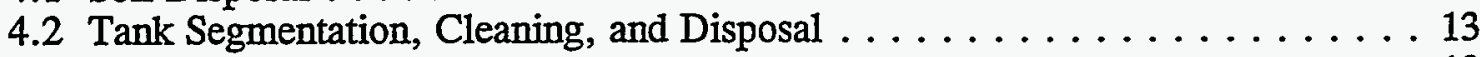

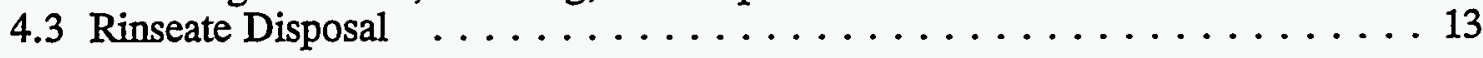

5.0 SUMMARY; CONCLUSIONS, and RECOMMENDATIONS $\ldots \ldots \ldots \ldots \ldots$

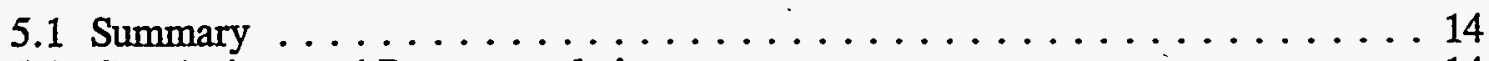

5.2 Conclusions and Recommendations $\ldots \ldots \ldots \ldots \ldots \ldots \ldots \ldots \ldots$

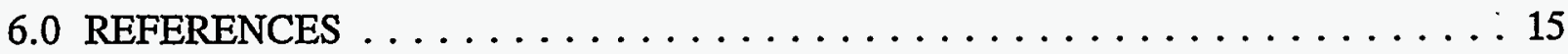




\section{FIGURES}

Figure

Page

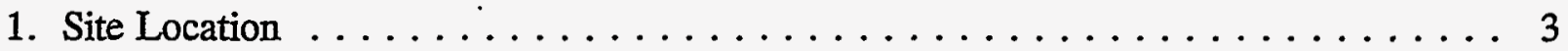

2. Tank Excavation and Sample Location $\ldots \ldots \ldots \ldots \ldots \ldots \ldots \ldots \ldots$

\section{TABLE}

Table

Page

1. Summary of Analytical Results For Tank Closure Activities $\ldots \ldots \ldots \ldots \ldots$

\section{APPENDICES}

\section{Appendix}

A. Notification of Closure - Environmental Protection Agency Form 7530-1

B. Laboratory Analytical Data Packets

C. Waste Manifests

D. Field Notes

E. Spill Notification Report

F. Landfill Disposal Documentation 


\section{ABBREVIATIONS AND ACRONYMS}

\begin{tabular}{ll} 
ASL & Analytical Services Laboratory \\
BN RP & Bechtel Nevada Remediation Projects \\
CFR & Code of Federal Regulations \\
CGA & Combustible Gas Analyzer \\
DOE/NV & U. S. Department of Energy/Nevada Operations Office \\
EPA & U. S. Environmental Protection Agency \\
ERD & Environmental Restoration Division \\
HPT & Health Protection Technician \\
LEL & Lower Explosive Limit \\
mg/kg & milligrams per kilogram \\
mg/l & milligrams per liter \\
NAC & Nevada Administrative Code \\
NDEP & Nevada Division of Environmental Protection \\
NTS & Nevada Test Site \\
OVM & Organic Vapor Monitor \\
PCB & Polychlorinated Biphenyl \\
ppb & parts per billion \\
ppm & part per million \\
RCRA & Resource Conservation and Recovery Act \\
REECo & Reynolds Electrical \& Engineering Co., Inc. \\
semi-VOCs & semivolatile organic compounds \\
TPH & Total Petroleum Hydrocarbons \\
UST & Underground Storage Tank \\
VOCs & Volatile Organic Compounds \\
& \\
\hline
\end{tabular}




\subsection{INTRODUCTION}

\subsection{INTRODUCTION}

The project site was identified as an abandoned Underground Storage Tank (UST) to be closed under the Department of Energy/Nevada Operations Office (DOE/NV) Environmental Restoration Division (ERD) Program during Fiscal Year 1993. The United States Environmental Protection Agency (EPA) requires that before permanent closure is completed an assessment of the site must take place (U.S. EPA 1994b). The Nevada Division of Environmental Protection (NDEP) requires assessment and corrective actions for a petroleum substance in the soil which exceeds 100 milligrams per kilogram (mg/kg)(NAC 1992b).

Subsequent to the tank removal, a hydrocarbon release was identified at the site. The release was reported to the NDEP by DOE/NV on November 30, 1993. Nevada Division of Environmental Management (NDEM) Case Number H931130E was assigned. This final closure report documents the assessment and corrective actions taken for the hydrocarbon release identified at the site. The Notification of Closure, EPA Form 7530-1 dated March 22, 1994, is provided in Appendix A. A 45-day report documenting the notification for a hydrocarbon release was submitted to NDEP on April 6, 1994 (REECo, 1994).

\subsection{PURPOSE AND SCOPE}

The purpose and scope of work for tank closure activities includes evaluating site conditions, closing the tank in accordance with all applicable regulations, and preparation of this report of findings. Closure of the UST site 12-13-1 was accomplished by completing the following activities:

- collection of a representative sample of the tank contents for laboratory analyses;

- removal and disposal of tank contents according to applicable regulations;

- excavation and removal of the tank and associated pipes;

- collection of soil samples at the bottom ends of the tanks for laboratory analyses to evaluate site conditions;

- segmentation and cleaning of the tank interior;

- collection and disposal of the rinseate generated from equipment and tank cleaning activities according to applicable regulations;

- crushing and disposal of the cleaned tank in a Class II Sanitary Landfill at the Nevada Test Site (NTS); 
- evaluation of the laboratory analytical results; and

- preparation of closure documentation for submission by DOE/NV to the NDEP.

\subsection{SITE DESCRIPTION}

The abandoned tank was located at the Area 12 Boiler House (Building 12-13) at the NTS, Nevada (Figure 1). The 5,680 liter (1,500 gallons) steel tank provided diesel fuel for a boiler located in Building 12-13. The tank was situated along the north side of the building (Figure 2). Approximate Nevada Coordinates for the site are N890,000 and E650,000 (from NTS Road and Facility Map, Holmes and Narver, 1986 update). Records indicate the tank was installed in February, 1962. No records were located identifying when the tank was no longer in service. The nearest water well (Well No. 8) is located approximately 12 kilometers (7.5 miles) southeast of the site (RSN, 1986). Depth to water in Well No. 8 is approximately 327 meters $(1,073$ feet $)$ below the land surface. 

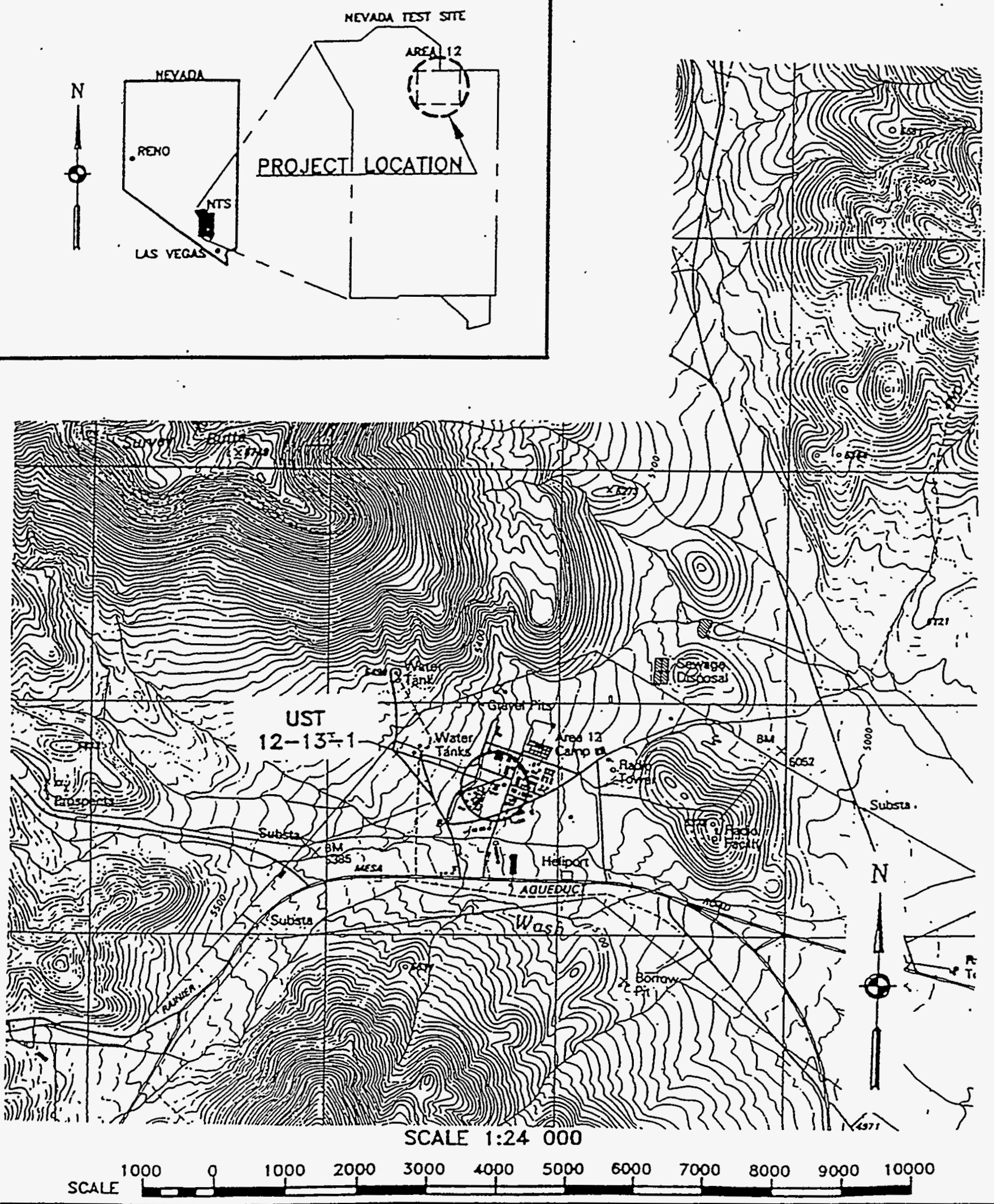

Bechtel Nevada

FIGURE 1 - SITE LOCATION

SOURCE: USGS RAINER MESA, NEVADA 7.5 MINUTE QUADRANGLE, 1986 


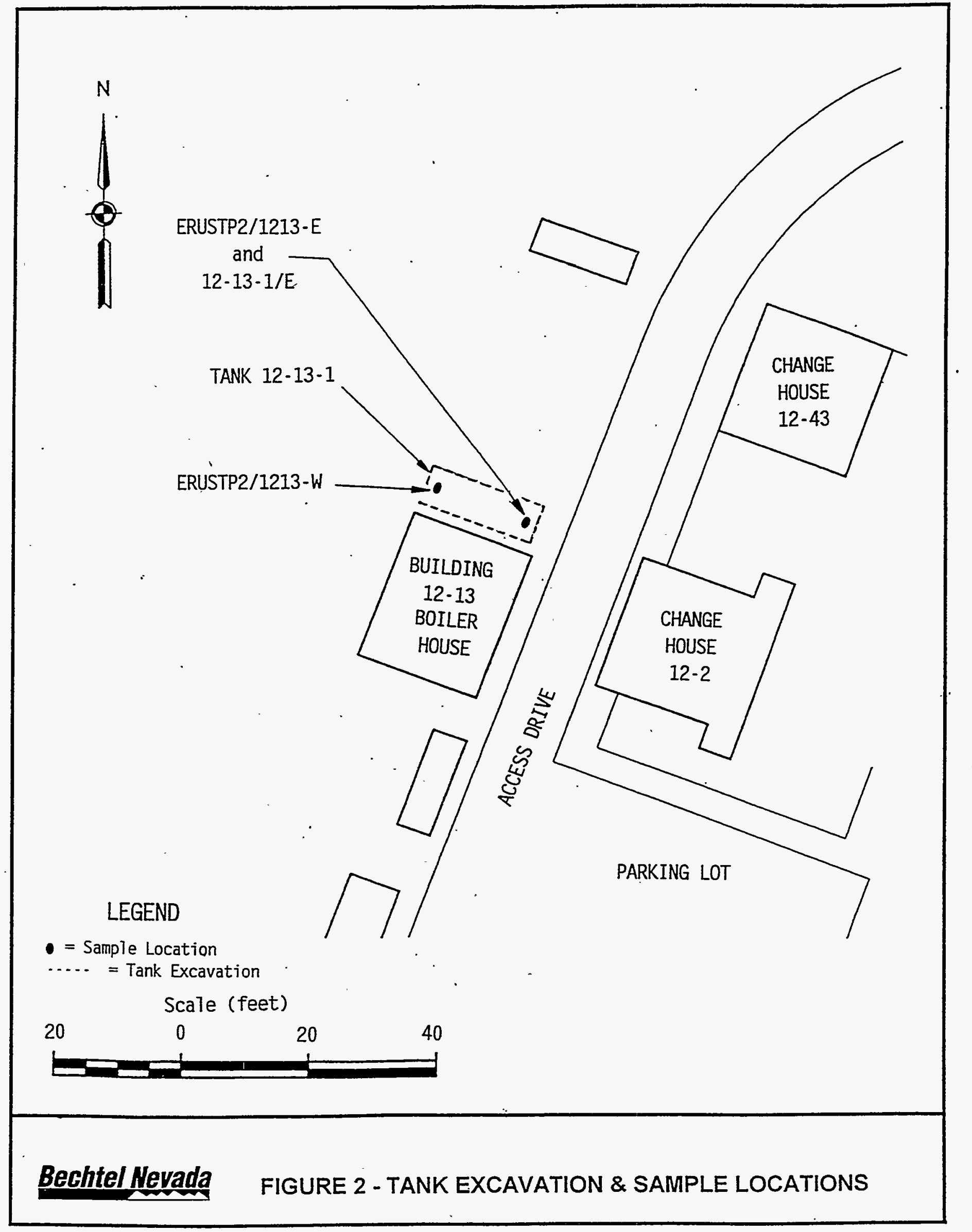




\subsection{TANK CLOSURE ACTIVITIES}

\subsection{PRODUCT MANAGEMENT}

\subsubsection{TANK SAMPLING AND RESULTS}

The tank was inspected and sampled on September 16, 1992. It was found to contain $71 \mathrm{~cm}$ (28 inches) of fluid which consisted of $63.5 \mathrm{~cm}$ ( 25 inches) of fuel product and $7.62 \mathrm{~cm}$ (three inches) of water. Since no documentation or physical controls for management of the tank contents (locks or security seals) were observed, process knowledge could not be used to characterize the tank contents. Samples were obtained using a new Coliwasa Sampler for both the organic and aqueous phase and analyzed for:

- Volatile Organic Compounds (VOCs), U.S. EPA Method 8240;

- Semi-VOCs, U.S. EPA Method 8270;

- Total Metals, U.S. EPA Method 6010

- Polychlorinated Biphenyls (PCBs), U.S. Method 8080;

- Total Petroleum Hydrocarbons (TPH), U.S. EPA Method 8015, Modified;

- $\mathrm{pH}$, U.S. EPA Method 9040/9045;

- Flashpoint, U.S. EPA Method 1010; and

- Gamma Activity, Reynolds Electrical and Engineering Company (REECo) Counting and Instrumentation Procedure.

The samples were placed into clean, laboratory-supplied jars with Teflon lids, sealed and labeled prior to cooling to approximately four degrees centigrade in an ice chest. The samples were transported to the REECo Analytical Services Laboratory (ASL) in Mercury, Nevada, following standard chain-of-custody procedures. The REECo Laboratory was the predecessor to Bechtel Nevada (BN) Analytical Services. 


\subsubsection{PRODUCT REMOVAL AND DISPOSAL}

Laboratory analytical results (Table 1 and Appendix B) indicated that the product within the tank was primarily diesel fuel $(913,000$ milligrams per liter [mg/l]). An estimated 3,785 liters (1,000 gallons) of product was removed from the tank on September 22, 1993. It was transported as a combustible liquid waste by Rollins Custom Environmental Transport to Los Angeles, California for recycling. Laboratory data packages are provided in Appendix B. Laboratory analytical results are summarized in Table 1. A copy of Waste Manifest Number 93221 is included in Appendix C.

Approximately 94 liters ( 25 gallons) of product remained in the tank and was pumped into a 208 liter (55 gallon) drum on October 20, 1993. The drum contents contained low concentrations of gasoline; therefore, it could not be disposed of in the Area 6 Hydrocarbon Landfill. The drum was removed on April 25, 1995 by the REECo Waste Management Department as hazardous waste. A copy of Waste Manifest Number 95086 is included in Appendix C.

\subsection{SOIL EXCAVATION AND TANK REMOVAL}

Prior to the start of tank excavation activities, the site was cleared for subsurface utilities by . reviewing engineering drawings, when available, and the use of Goldak metal detection equipment. Hand excavation was used to confirm the location of suspected utilities prior to excavating with a backhoe. Hand excavation was done using non-sparking shovels, picks, etc.

Before excavation began, the interior atmosphere of the tank was made inert using nitrogen gas. A Combustible Gas Analyzer (CGA) was used to confirm that a lower explosive limit (LEL) of less than 20 percent and an oxygen $\left(\mathrm{O}_{2}\right)$ content of less than seven percent was achieved: The backhoe and tank were electrically grounded during all excavation activities.

The tank excavation and removal occurred on October 20, 1993. A backhoe was used to excavate sufficient soil above and around the tank to allow for removal with a crane. The tank was marked with spray paint to indicate the NTS Tank Number. A radiologic "Green Tag" was issued to indicate that the tank was screened and found to be below radiological release limits prior to transporting to the NTS U10c Landfill Segmentation Pad. Soils that were removed from the excavation were placed on plastic. The pile was covered with plastic and secured with sandbags at the end of each work day.

\subsubsection{SAMPLING ACTIVITIES}

Prior to sample collection, the backhoe bucket was steam cleaned over a 208 liter (55 gallon) drum to contain the rinseate. The backhoe bucket was also cleaned between each sample point to reduce the potential of cross-contamination. A sample of the rinseate was collected on October 20, 1993. 
Soil samples were collected on October 20, 1993 from beneath the tank at each end at a depth of 2.7 meters (nine feet). The samples were collected approximately 0.3 meters (one foot) below the tank bottom. Samples were analyzed for TPH using U.S. EPA Method 8015, Modified. A headspace sample was also collected at each sample location. Soil samples were collected from the clean backhoe bucket using a stainless steel scoop.

The soil pile that was generated during the excavation was sampled on November 9, 1993. One composite soil sample from three locations within the pile was collected using a stainless steel hand auger at an approximate depth of 0.9 meters (three feet) into the pile. The sample was analyzed for TPH using U.S. EPA Method 8015, Modified.

The samples were placed into clean, laboratory-supplied jars with Teflon lids, sealed and labeled prior to cooling to approximately four degrees centigrade in an ice chest. The samples were subsequently transported to the REECo ASL, Mercury, Nevada, following standard chain-of-custody procedures. The REECo Laboratory was the predecessor to the (BN) Analytical Services. 
TABLE 1 - SUMMARY OF ANALYTICAL RESULTS FOR TANK CLOSURE ACTIVITIES

\begin{tabular}{|c|c|c|c|c|c|c|c|c|c|c|}
\hline 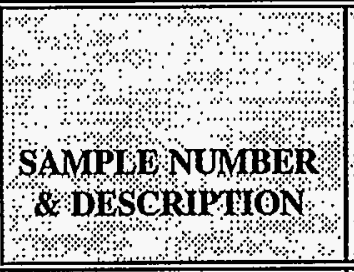 & & 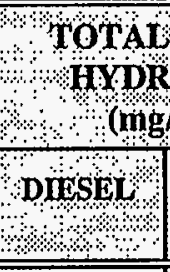 & 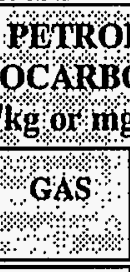 & ONSW & 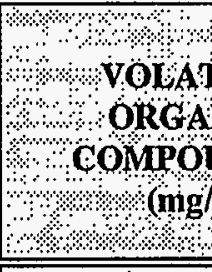 & & 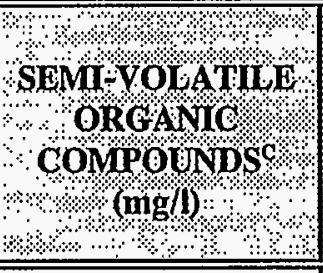 & 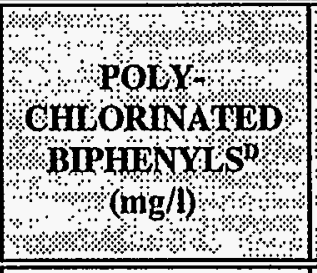 & 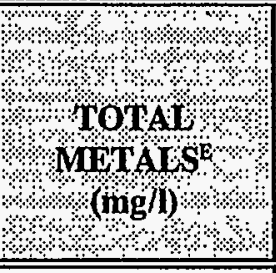 & 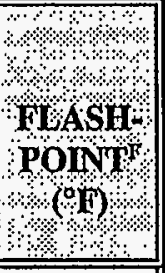 \\
\hline $\begin{array}{l}12-13-1-\mathrm{TK} \\
\text { (tank contents -organic } \\
\text { phase) }\end{array}$ & $9 / 16 / 92$ & 913,000 & 167 & $<10.0$ & $\begin{array}{l}\text { Benzene } \\
\text { Toluene } \\
\text { Ethylbenzene } \\
\text { Total Xylene }\end{array}$ & $\begin{array}{l}110 \\
690 \\
410 \\
1,690\end{array}$ & $\begin{array}{l}\text { 2-Methyl- } \quad 1.0 \\
\text { naphthalene. }\end{array}$ & $<2.5$ & Mercury $\quad 0.02$ & 189 \\
\hline $\begin{array}{l}12-13-1-\mathrm{TK} \\
\text { (tank contents - } \\
\text { aqueous phase) }\end{array}$ & $9 / 16 / 92$ & 14,200 & 24 & $<0.5$ & $\begin{array}{l}\text { Acetone } \\
\text { 2-Butanone } \\
\text { Benzene } \\
\text { Toluene } \\
\text { Ethylbenzene } \\
\text { Total Xylene }\end{array}$ & $\begin{array}{l}0.310 \\
0.094 \\
0.180 \\
0.340 \\
0.076 \\
0.340\end{array}$ & ND & NA & ND & 164 \\
\hline $\begin{array}{l}\text { ERUSTP2/ } \\
\text { 1213-W } \\
\text { (west bottom) }\end{array}$ & $10 / 20 / 93$ & $<10.0$ & $<5.0$ & $<10.0$ & $\mathrm{~N}$ & & $\mathrm{NA}$ & NA & NA & NA \\
\hline $\begin{array}{l}\text { ERUSTP2 } f \\
\text { 1213-E (east bottom) }\end{array}$ & $10 / 20 / 93$ & 116 & $<5.0$ & $<10.0$ & $\mathrm{~N}$ & & $\mathrm{NA}$ & NA & NA & $\mathrm{NA}$ \\
\hline $\begin{array}{l}\text { 12-13/PILE. } \\
\text { (soil pile sample) }\end{array}$ & $11 / 9 / 93$ & 671 & $<5.0$ & $<10.0$ & $\mathrm{~N}$ & & NA & $\mathrm{NA}$ & NA & NA \\
\hline $\begin{array}{l}\text { ERUSTP2/1213- } \\
\text { RINSE } \\
\text { (rinseate sample) }\end{array}$ & $10 / 20 / 93$ & 0.730 & $<5.0$ & $<0.5$ & $\mathrm{~N}$ & & NA & NA & NA & $\mathrm{NA}$ \\
\hline
\end{tabular}


Notes:

${ }^{A}$ United States Environmental Protection Agency (US EPA) Method SW-846, 8015 (Modified)

B US EPA Method 8240

C US EPA Method 8270

D US EPA Method 8080

E US EPA Method 6010

F Pensky Martin Method

NA-Not analyzed

ND - Not detected

${ }^{\circ} \mathrm{F}$ - degrees Fahrenheit

$\mathrm{mg} / \mathrm{kg}$ - milligrams per kilogram

mg/l - milligrams per liter

Action Levels:

Total Petroleum Hydrocarbons: Nevada Administrative Code 459.9973 TPH Action Level of 100 parts per million. 


\subsubsection{ANALYTICAL RESULTS}

The rinseate generated from decontaminating the backhoe bucket contained TPH as diesel at a concentration of $0.730 \mathrm{mg} / 1$ (Sample ERUSTP2/1213-RINSE). TPH as diesel were detected in the soil sample from the east end of the excavation bottom at a concentration of $116 \mathrm{mg} / \mathrm{kg}$ (Sample ERUSTP2/1213-E). TPH as diesel were not detected $(<10 \mathrm{mg} / \mathrm{kg}$ ) in the sample collected from the west excavation bottom (Sample ERUSTP2/1213-W). Laboratory analytical results are summarized in Table 1.

The soil pile sample had a TPH concentration as diesel of $671 \mathrm{mg} / \mathrm{kg}$ (12-13/PILE). TPH as gasoline and oil were not detected in the soil samples collected below the tank bottom or stockpile (Table 1).

\subsubsection{OBSERVATIONS}

The overall integrity of the tank appeared good. No dents, holes or pits were observed. Hydrocarbon odors were not observed. Soils removed from the excavation were slightly moist and well graded. Field notes are provided in Appendix D.

Headspace readings were collected using a Thermo Environmental Instruments, Inc., organic vapor monitor (OVM) Model 580B using an 11.8 electron volt lamp. The OVM was calibrated to 100 parts per million (ppm) Isobutylene at the beginning of each field day. The readings were collected by placing approximately 100 grams of soil into an airtight bag. After approximately 30 minutes the OVM tip was inserted into the bag to measure the volatile headspace. The highest reading was then documented in the field notebook. The sample taken at the west end of the excavation was less than six ppm and the sample taken at the east end of the excavation was less than five ppm. The headspace reading from the soil pile was 17.1 ppm. Field notes are provided in Appendix D.

\subsubsection{BACKFILL}

The excavation was backfilled on October 20, 1993 to preserve the structural integrity of Building 12-13 which was slightly undermined during the excavation and tank removal. Pea gravel was used to fill the excavation.

\subsection{SPILL NOTIFICATION REPORT}

The sample from beneath the east end of the tank exceeded the NAC 459.9973 Action Level of $100 \mathrm{mg} / \mathrm{kg}$ TPH. Notification of the release was reported to the NDEP by DOE/NV on November 30, 1993 and NDEM Case Number H931130E was assigned. The Spill . Notification Report is provided in Appendix E. 


\subsection{REMEDIAL ACTIVITIES}

\subsection{EXCAVATION AND SAMPLING ACTIVITIES}

The pea gravel that was used to backfill the excavation on October 20, 1993 was removed from above and around the tank and piping on December 7 and 8, 1994. Approximately 5.4 cubic meters (seven cubic yards) of impacted soil were removed from the east end of the excavation on December 8, 1994. The impacted soils were stockpiled on plastic adjacent to the tank excavation and kept separate from the pea gravel. Soil was covered with plastic and secured with sandbags at the end of each day.

One soil sample was collected from below the east end of the tank at a depth of approximately three meters (ten feet) below ground surface. This sample was taken to verify the removal of all impacted soils. One composite sample from two random locations was collected from the soil stock pile for characterization. Both samples were collected on December 8, 1994. Samples were analyzed for TPH as diesel using EPA Method 8015 Modified.

Samples were collected using a clean stainless steel scoop. They were placed into clean laboratory-supplied jars with Teflon lids, sealed and labeled prior to cooling to approximately four degrees centigrade in an ice chest. The samples were transported to the REECo Laboratory in Mercury, Nevada, following standard chain-of-custody procedures.

\subsection{ANALYTICAL RESULTS}

TPH as diesel were not detected above the laboratory detection limit $(<10 \mathrm{mg} / \mathrm{kg})$ for soil sample 12-13-1/E collected from the east excavation bottom. The sample taken from the soil pile (12-13-1/SP1) had a diesel concentration of $20 \mathrm{mg} / \mathrm{kg}$. The laboratory analytical data is provided in Appendix B (ACS Packet No. FY94-12-021).

\subsection{OBSERVATIONS}

Excavated soil consisted of damp, gravelly sand to sandy gravel. No hydrocarbon odors or soil staining was observed. Field notes documenting remedial activities are found in Appendix D. 


\subsection{BACKFILL}

The tank excavation was backfilled for the second time on March 21, 1995 with the pea gravel that was removed from the excavation on December 7 and 8,1994 . The excavation was capped with nearby clean native soils. Associated tank piping was cut and sealed with grout. 


\subsection{RESIDUALS MANAGEMENT}

\subsection{SOIL DISPOSAL}

The soil pile generated during the tank removal excavation on October 20,1993, consisted of approximately 30.6 cubic meters ( 40 cubic yards). It was removed from the site on January 31, 1994, and transported to the Area 6 Hydrocarbon Landfill for disposal. The soil pile generated during the remedial activities on December 8,1994 , consisted of approximately 5.4 cubic meters (7 cubic yards). It was removed from the site May 4, 1995, and transported to the Area 6 Hydrocarbon Landfill for disposal. Soil disposal documentation is provided in Appendix F.

\subsection{TANK SEGMENTATION, CLEANING, AND DISPOSAL}

Tank 12-13-1 was segmented using explosive cutting tape on February 22, 1994. Prior to segmentation, the tank was confirmed to be inert using a CGA. After the tank was opened, it was cleaned using high pressure hot water. The rinseate was placed in new or reconditioned 208 liter (55-gallon) drums. Once clean, the tank was subsequently crushed and buried in the NTS U10c Class II sanitary landfill.

\subsection{RINSEATE DISPOSAL}

The rinseate from the sampling activities conducted on October 20,1993, was removed from the site on December 1, 1994 and pumped into the Area 23 Sewage Lagoon System. Rinseate generated from the cleaning of the interior of the tank was solidified and transported to the Area 6 Hydrocarbon Landfill for disposal on April 3, 1995. 


\subsection{SUMMARY, CONCLUSIONS, AND RECOMMENDATIONS}

\subsection{SUMMARY}

Analytical results from the soil sample collected below the tank on the east end on October 20 , 1993, exceeded the NAC Action Level of $100 \mathrm{mg} / \mathrm{kg}$. A diesel concentration of $116 \mathrm{mg} / \mathrm{kg}$ was detected at a depth of 2.7 meters (nine feet) below ground surface.

Additional excavation and characterization was conducted on December 7 and 8, 1994. Approximately 3.8 cubic meters (five cubic yards) of hydrocarbon impacted soil was removed from the eastern portion of the excavation. TPH as diesel concentrations from the remedial excavation was not detected in the bottom sample collected.

An approximate total of 36 cubic meters ( 47 cubic years) of soil generated from the tank removal and remediation activities were disposed of into the Area 6 Hydrocarbon Landfill.

\subsection{CONCLUSIONS AND RECOMMENDATIONS}

Based on analytical results, soils with elevated hydrocarbon concentrations detected in the vicinity of Tank 12-13-1 were excavated during the remedial activities. The site is recommended to be closed without further action based upon the site observations and analytical data collected from the additional soil excavation and characterization. 


\subsection{REFERENCES}

Holmes and Narver, 1986, NTS Road and Facility Map. 1986 Update

NAC 1992a, Nevada Administrative Code, Chapter 459, Hazardous Materials, Storage Tanks, 459.9972(4)(a).

NAC 1992b, Nevada Administrative Code, Chapter 459, Hazardous Materials, Storage Tanks, 459.9973.

NAC 1994, Nevada Administrative Code, Chapter 459, Water Controls, Notification of Release of Pollutant, 459A.347.

REECo, 1994, 45-Day Report For Nevada Division of Emergency Management (NDEM) Case Number H931130E, Area 12 Boiler House, Underground Storage Tank Number 12-13-1. Reynolds Electrical \& Engineering, Co., March 31, 1994.

RSN, 1986, Nevada Site Development Plan, Part I, May 1986.

U.S. EPA, 1994a, Code of Federal Regulations, 40 CFR 280.71, Technical Standards and Corrective Action Requirements for Owners and Operators of Underground Storage Tanks. Permanent Closure and Changes-in-Service.

U.S. EPA, 1994b, Code of Federal Regulations 40 CFR 280.72, Technical Standards and . Corrective Action Requirements for Owners and Operators of Underground Storage Tanks. Assessing the Site at Closure or Change-in-Service.

U.S. EPA, 1994c, Code of Federal Regulations 40 CFR 280.50 and 280.53, Technical Standards and Corrective Action Requirements for Owners and Operators of Underground Storage Tanks, Subpart E - Release Reporting. Investigation, and Confirmation. 



\section{APPENDIX A \\ NOTIFICATION OF CLOSURE ENVIRONMENTAL PROTECTION AGENCY \\ FORM 7530-1}





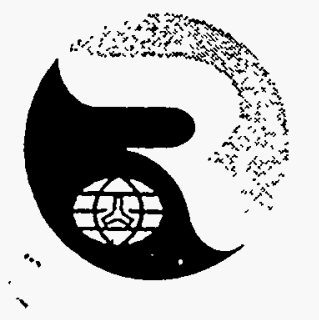

\title{
Reynolds Electrical \& Engineering Co., Inc.
}

\author{
Post Office Box 98521 - Las Vegas, NV 89193-8521
}

IN AEPLY REFER TO:

592-07-19

March 22, 1994

Donald R. Elle, Director

Environmental Protection Division

DOE Nevada Operations Office

Post Office Box 98518

Las Vegas, NV 89193-8518

NOTIFICATION OF CLOSURE FOR TWELVE ENVIRONMENTAL RESTORATION

PROGRAM ABANDONED UNDERGROUND STORAGE TANKS (USTS)

Enclosed is the Environmental Protection Agency Form 7530-1, Notification for Underground Storage Tanks, which identifies twelve abandoned USTs that were permanently closed by removal prior to March 24, 1994. The tanks were required to be removed within one year of the March 24, 1993, notification to the Nevada Division of Environmental Protection (NDEP). The permanent7y closed tanks are:

$$
\begin{array}{llll}
11-1-1 & 12-8-1 & 12-8-2 & 12-9-1 \\
12-9-2 & 12-13-1 & 23-110-1 & 23-112-1 \\
25-3102-3 & 25-3205-1 & 27-5170-1 & 27-5310-1
\end{array}
$$

Copies of the laboratory analytical results of soil samples collected below the bottom end of the tanks are enclosed and summarized in Table 1. Analytes for the soil samples were determined from evaluation of the analytical results from the contents of each of the tanks (example: Tank 11-1-1 contained diesel fuel with volatile organic compounds and metals not normaily associated with diesel fuels [1,1,1-Trichloroethane and Mercury]; therefore the soil was analyzed for volatile organic compounds and Toxicity Characteristic Leaching Procedure metals). For tanks $12-8-1,12-8-2,12-9-1$, and 12-9-2, additiona 1 analytes were requested at the time of the sampling of the soil because characterization of the tanks' content were not completed at the time of the soil sampling.

NDEP has been notified that 12-13-1, 25-3102-3, 27-5310-1, and 27-5170-1 tank sites have Total Petroleum Hydrocarbon concentrations in soil, which exceed the $100 \mathrm{mg} / \mathrm{kg}$ Action Level. Additional soil sampling at the indicated sites is required to evaluate site conditions. Work Plans are being developed for these sites. 
Donald R. Elle

592-07-19

Page 2

March 22, 1994

If you have any questions, please contact Dave Madsen at 295-7211.

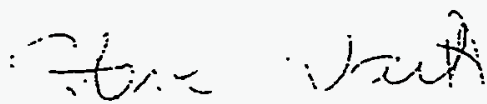

Robert L. Dodge, Manager

Environmental Restoration \&

Technology Development Department

RLD:DDM:ap

Enclosure

As stated

cy $w / e n c l$.

Central Files, M/S 530 THRU

Executive Office, M/S 555

J. L. Appenzeller-Wing, DOE/NV, M/S 505

S. A. Mellington, DOE/NV, M/S 505

J. D. Stewart, DOE/NTSO, M/S 701

B. Yoerg, DOE/NTSO, M/S 701

cy $w / 0$ encl.

H. W. Dickson, REECo, M/S 612

J. H. Wuellner, REECo, M/S 175 
ENVIRONMENTAL RESTORATION PROGRAM

ABANDONED UNDERGROUND STORAGE TANKS (PHASE 2 - FY93) NOTIFICATION FOR UNDERGROUND STORAGE TANKS 

Page 1 of 11

Attention: UST Coordinator

Division of Environmental $P$ ection

Dept. of Conservation \& Natural Resources

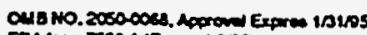

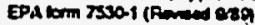

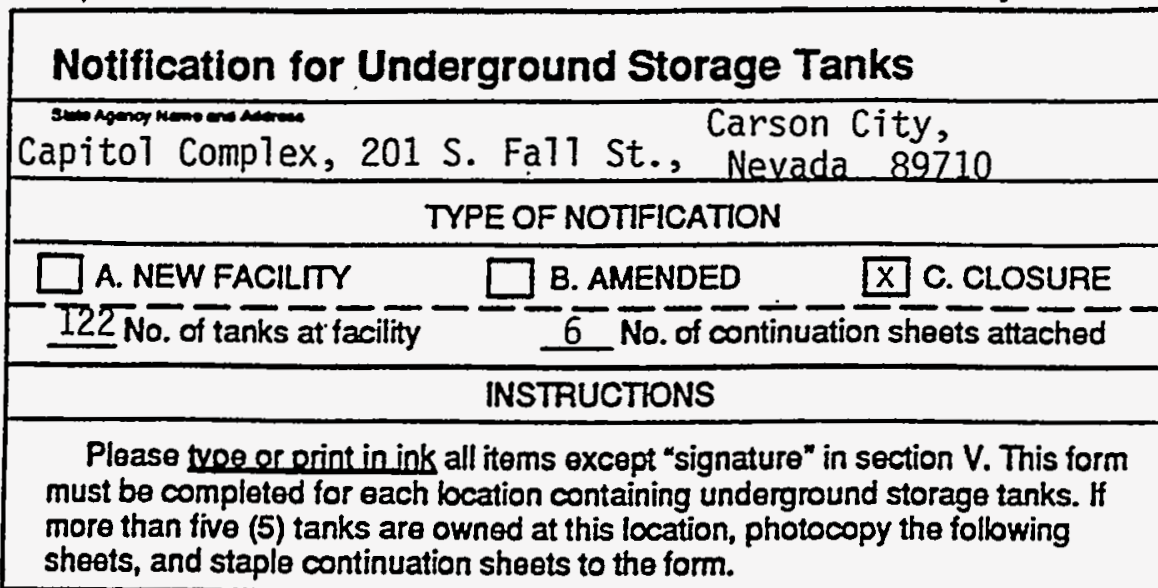

STATE USE ONLY

ID NUMBER

DATE RECEIVED

A. Date Entered into Computer

B. Data Entry Clerk Initials

C. Owner Was Contacted to

Clarity Responses. Comments

\section{GENERAL INFORMATION}

1

Hotification is required by Fedarel inw for all underground tanke that have been used to edore regulated subetances elnce January 1, 1974, that are in the ground as of May B, 1986, or that are brought Into use aftor Way B, 1986. The Informatlon requeted is tequired by Section 9002 of the Resource Consarvation and Recovery Act, (RCRA), as amended.

The primary purpose of this notification program is to beate and evaluate underground tanks that store or have stored peteleten of.hazadous substances. It is expected that the intomation you provide will be based on reasonably available records, or in the absence of such records, your knowledge, belief, of recollection.

Who Uust Mollty? Section 9002 of RCRA, as amended, requires that, unless oxempted, owners of underground tanks that store regulated substances must notity designated State or local apencies of the existence of their tanks. Owmer moans-

a) in the case of an underground storage tank in use on November B. 1984, or brought into use atter that date, amy person who owns an underground storage tank used for the storage, use, or dispensing of regulated substances, and

b) in the case of any underground storage tank in use bofore November 8 . 1984, but no longer is use on that date, any person who owned such tank immediately before the discontinuation of its use.

c) if the State agency so requires, any facilin thar has undergone any changes to facility information or ank system stanus (only amended tank information needs to be inciuded).

Whal Tanks Are included? Underground storage tank is defined as any one or combination of tanks that (1) is used to contain an acamulation of "regulated substances," and (2) whose volume (including connected underground piping) is

$10 \%$ or more beneath the ground. Some examples are underground tanks storing:

1. Gasoline, used oil, or diesel fuel, and 2 industrial solvents, pesticides.

horbicides or fumigants.

What Tanks Are Excluded? Tanks removed irom the ground are not subject to notification. Other tanks exduded from notification are:

1. farm or residential tanks of 1,100 gallons or less capacity used for storing motor fuel for noncommercial purposes:

2 tanks used tor storing heating oil for consumpove use on the premises where stored:
3. soptic taniks:

4. pipoline facitities (inctuding gathering lines) regutated under the Natural Gas Pipoline Safety Act of 1968, or the Hazardous Liquid Pipeline Salety Act of 1979, or which is an intrastate pipoline facility regulated uxder State laus:

3. surtace impoundiments, pirs, ponds, or lagoons:

6. storm water or waste wator colloction systoms:

T. föin-throught procisis tainks:

8: liquid-traps or associated gahtering lines dirocty related to oil or gas producion and gathering operations;

9. storage tanks sinuated in an underoround area (such as a basement, collar, mineworking. drith. shat, or tunnel) if the storage tank is siubato upon or above the surtace of the floor.

What Substances Are Covered? The notification requirements apply to under ground storage tanks that contain regulatod substancos. This inctudos any substance defined as hazardous in secion 101 (14) of the Comprehonsive Environmental Response, Compensation and Liability Act of 1980 (CERCLA), with the excoption of those substances regulated as hazardous waste under Subtido C o RCRA. It also includes petroleum, e.g., crude od or any traction thereof which is Fquid at standard conditions of temperature and pressure (60 dogrees Fahrenheit and 14.7 pounds por square inch absolute).

Whar To Hatity? Send completed forms to:

Whan To Notlfy? 1. Owners of underground sworage tanks in use or that have been taken out of operation after tanuary 1, 1974, but still in the ground, must notify by May 8. 1986. 2 Owners who bring underground storage taniks into use after May 8,1986 , must notify within 30 days of bringing the tanks into use. 3. If the State requires notitication of any amendments to facitity send intomation to State agency immodiately.

Penalties: Any owner who knowingty fails to notify or submits fales intormation shall be subject to a civil penalty not 20 axceed $\$ 10,000$ for each Iank for.which rexifieation is not given of for which faise information is cubmitted.

\section{OWNERSHIP OF TANK(S)}

Owner Namp peorporation. Individual, Public Agency, or Oher Entity DOE Nevada Operations Office simet Addos

\section{Post Office Box 98518}

\begin{tabular}{|c|c|}
\hline $\begin{array}{ll}\text { Lay } & \\
\text { Las Vegas } & \mathrm{NV}\end{array}$ & $\begin{array}{c}\text { 21PCode } \\
89193-8518\end{array}$ \\
\hline Clark & \\
\hline $\begin{array}{l}\text { (702) 295-1860 } \\
\text { (7) }\end{array}$ & \\
\hline
\end{tabular}

\section{LOCATION OF TANK(S)}

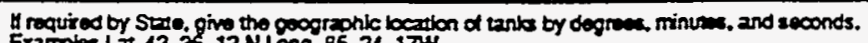
Examplex LII 42, 36. $12 \mathrm{NLOng}$. 25, 24. $17 \mathrm{~W}$

$$
\text { Latitude Not applicable Longitude Not Applic: }
$$

$$
\text { (H sam as Section 1, matk bex here } \square \text { ) }
$$

Faciry Nam or Company She identiflex, as apolibable

Nevada Test Site

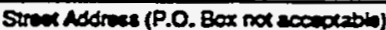

Highway 95

\begin{tabular}{|c|c|c|}
\hline Cy & Sino & $20 \times 000$ \\
\hline Mercury & Nv & 89093 \\
\hline $\begin{array}{l}\text { Nouny } \\
\text { Nye }\end{array}$ & & \\
\hline
\end{tabular}




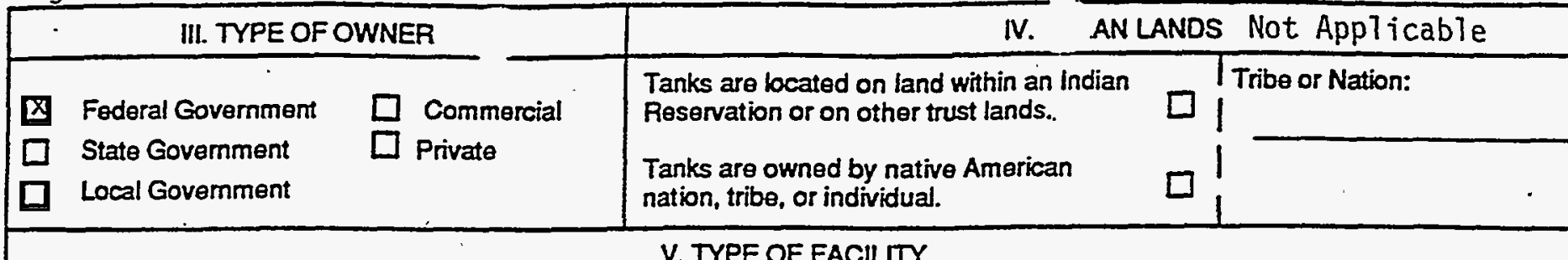

\section{TYPE OF FACILITY}

Select the Appropriate Facility Description

Gas Station

Petroleum Distributor

Air Taxi (Airline)

Aircraft Owner

Auto Dealership
Railroad

XX Federal - Non-Military

Foderal - Military

Industrial

Contractor
Trucking/Transport

Utilities

Residential

Farm

Other (Explain)

\section{CONTACT PERSON IN CHARGE OF TANKS}

\begin{tabular}{|ccc} 
Name & Job Title & $\begin{array}{c}\text { Address } \\
\text { D.R. ElTe }\end{array}$ \\
& Director & $\begin{array}{l}\text { Env. Protection Division } \\
\text { Post Office Box } 98518 \\
\text { Las Vegas, NV 89193-8518 }\end{array}$ \\
\hline & VII. FINANCIALRESPONSIBILITY
\end{tabular}

I have met the financial responsibility requirements in accordance with 40 CFR Subpart $H$

Phone Number (Include Area Code)

(702) 295-1433

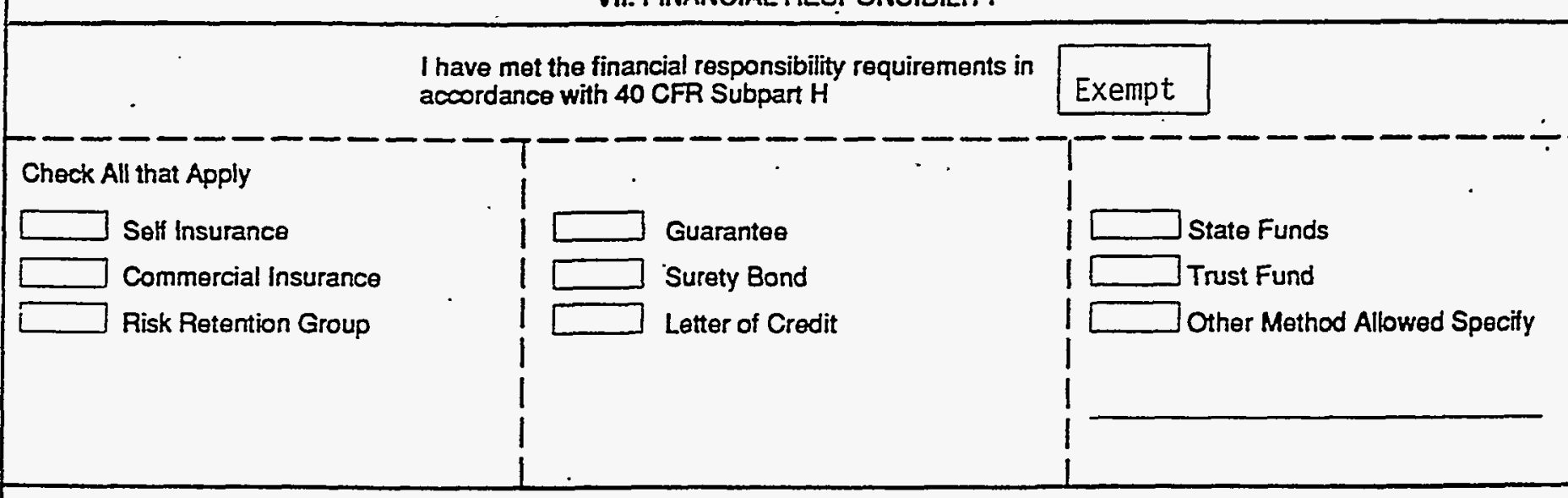

VIII. CERTIFICATION (Read and sign after completing all sections)

I certify under penaity of law that I have personally examined and am familiar with the information submitted in this and all attached documents, and that based on my inquiry of those individuals immediately responsible for obtaining the information, I believe that the submitted information is true, accurate, and complete.

Name and official title of owner

or owner's authorized representative (Print)

Donald R. Elle, Director
Signature

Date Signed

EPA estimates public reporting burden for this form to average 30 minutes per response including time for reviewing instructions, gathering and maintaining the data needed and completing and reviewing the form. Send comments regarding this burden estimate to Chief, Information Policy Branch PM-223, U.S. Environmental Protection Agency, 401 M Street, Washington D.C. 20460, marked "Attention Desk Officer for EPA." This form amends the previous notification form as printed in 40 CFR Part 280, Appendix I. Previous editions of this notification form may be used while supplies last. 
IX. DESCRIPTION OF L. .UERGROUND STORAGE TANKS (Complete fur each tank at this location.)

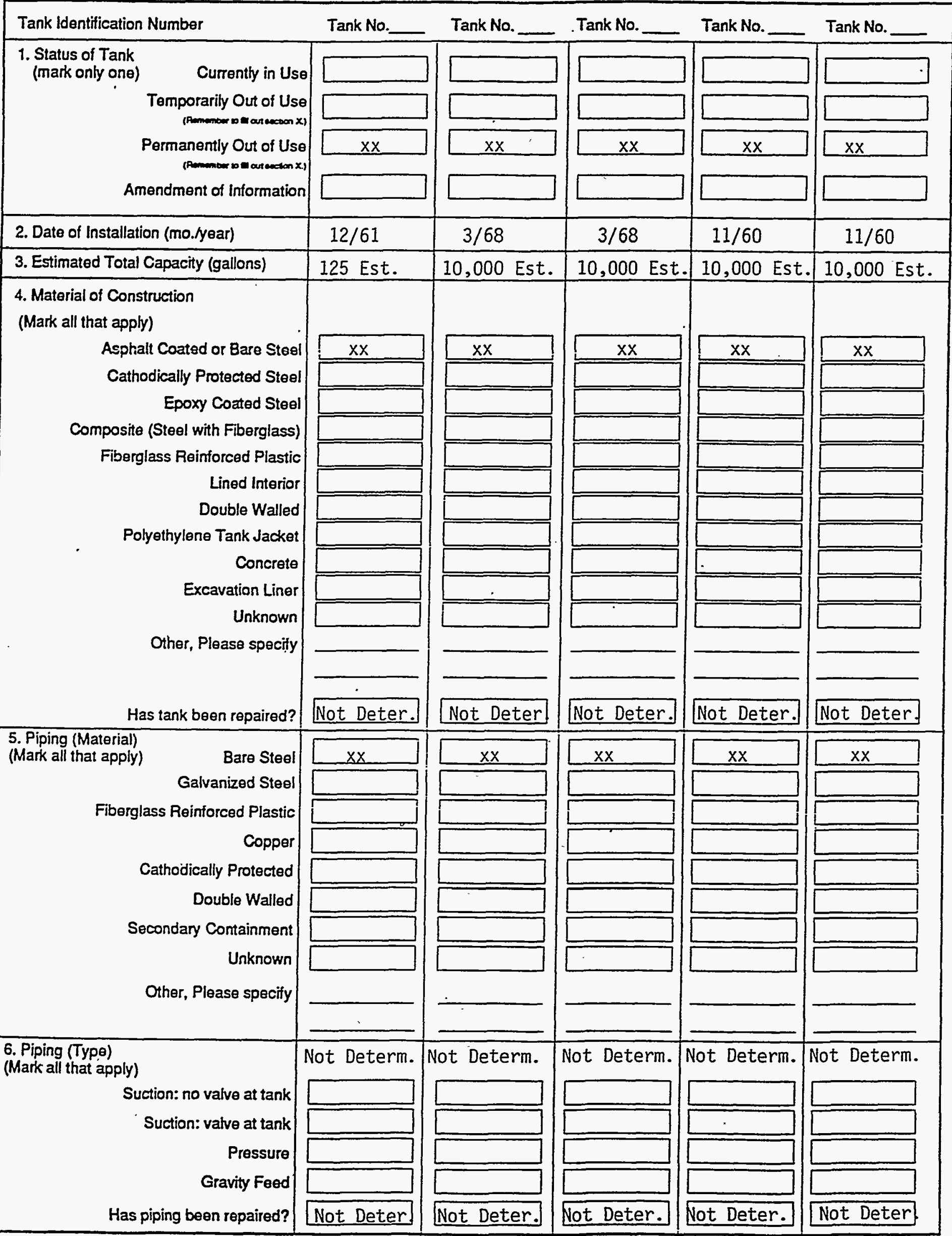


Page 4 of 11

12-13-1

23-110-1

$23-1^{\text {in }}-1$

25-3102-3

25-3205-1

IX. DESCRIPTION OF w.NDERGROUND STORAGE TANKS (Complate iur each tank at this tocation.)

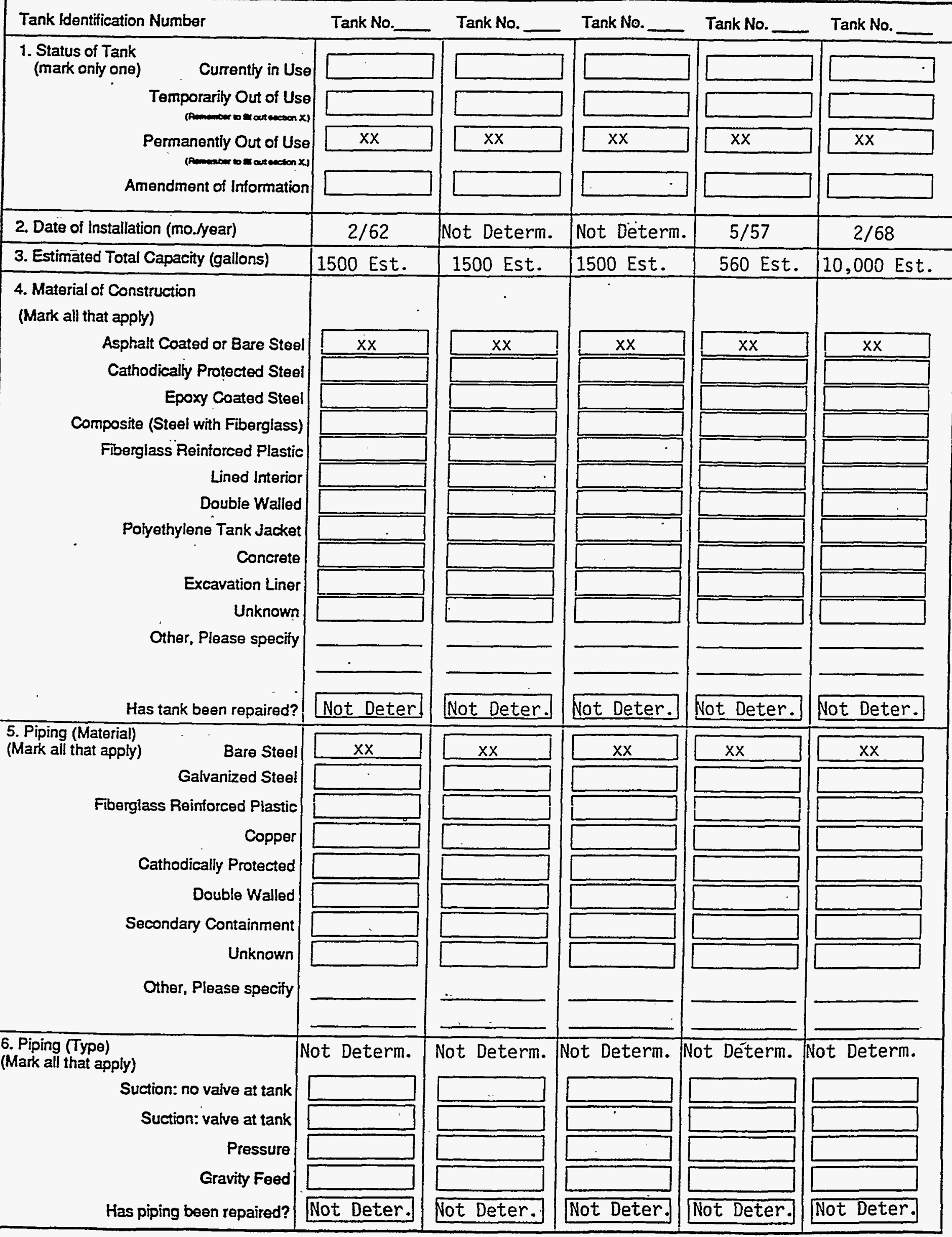


IX. DESCRIPTION OF C. .OERGROUND STORAGE TANKS (Complete tor each tank at this location.)

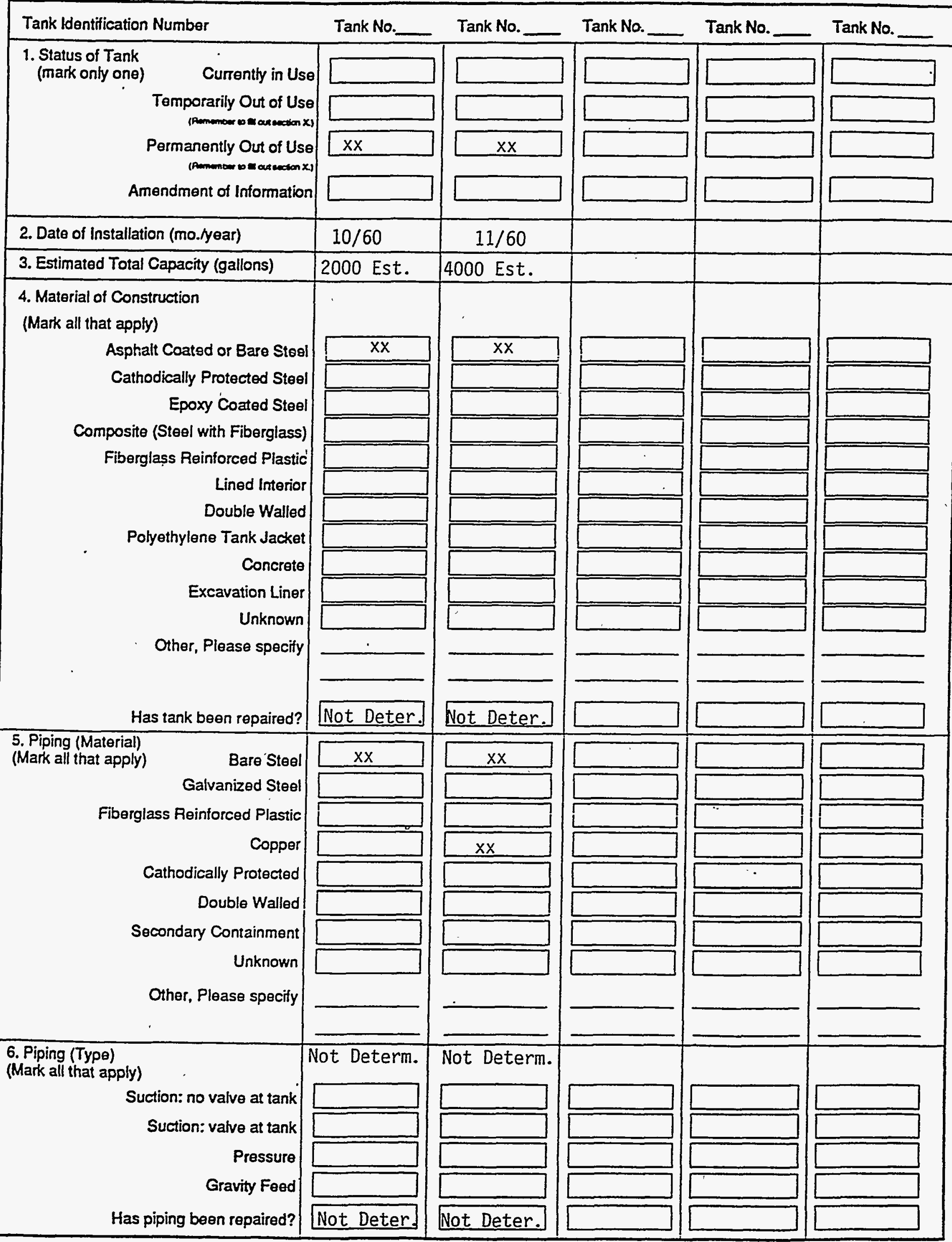


rage 0 or 11

$11-1-1$

$12-8-1$

$12-8-2$

$12-y-1$ $12-9-2$

Tank Identification Numoer

7. Substance Currently or Last Stored In Greatest Quantity by Volume Gasoline

Diesel

Gasohol

Kerosene

Heating Oil

Used Oil

Other, Please specify

Tank No

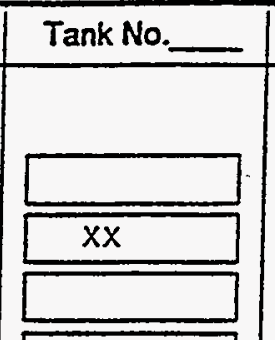

Tank Nr $=$ Tank No.

Tank No.

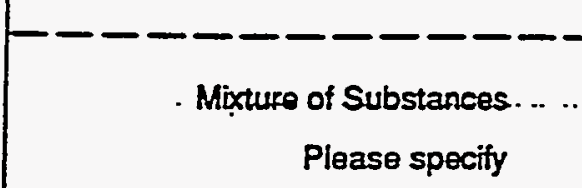

$---\infty-\infty-1-1$
Mixture of Substances
Please specify

Hazardous Substance

CERCLA name and/or,

CAS number

$X$ TANKS OUT OF USE, OR CHANGE IN SERVICE

1. Closing of Tank

A. Estimated date last used

Not Determ. Not Determ. Not Determ. Not Determ. Not Determ. (mo.ddayhyear)

B. Estimate date tank closed (mo.Jday/year)

C. Tank was removed from ground

D. Tank was closed in ground

E Tank filled with inert material

Describe

F. Change in service

2. Site Assessment Completed

Evidence of a leak detected

No

No

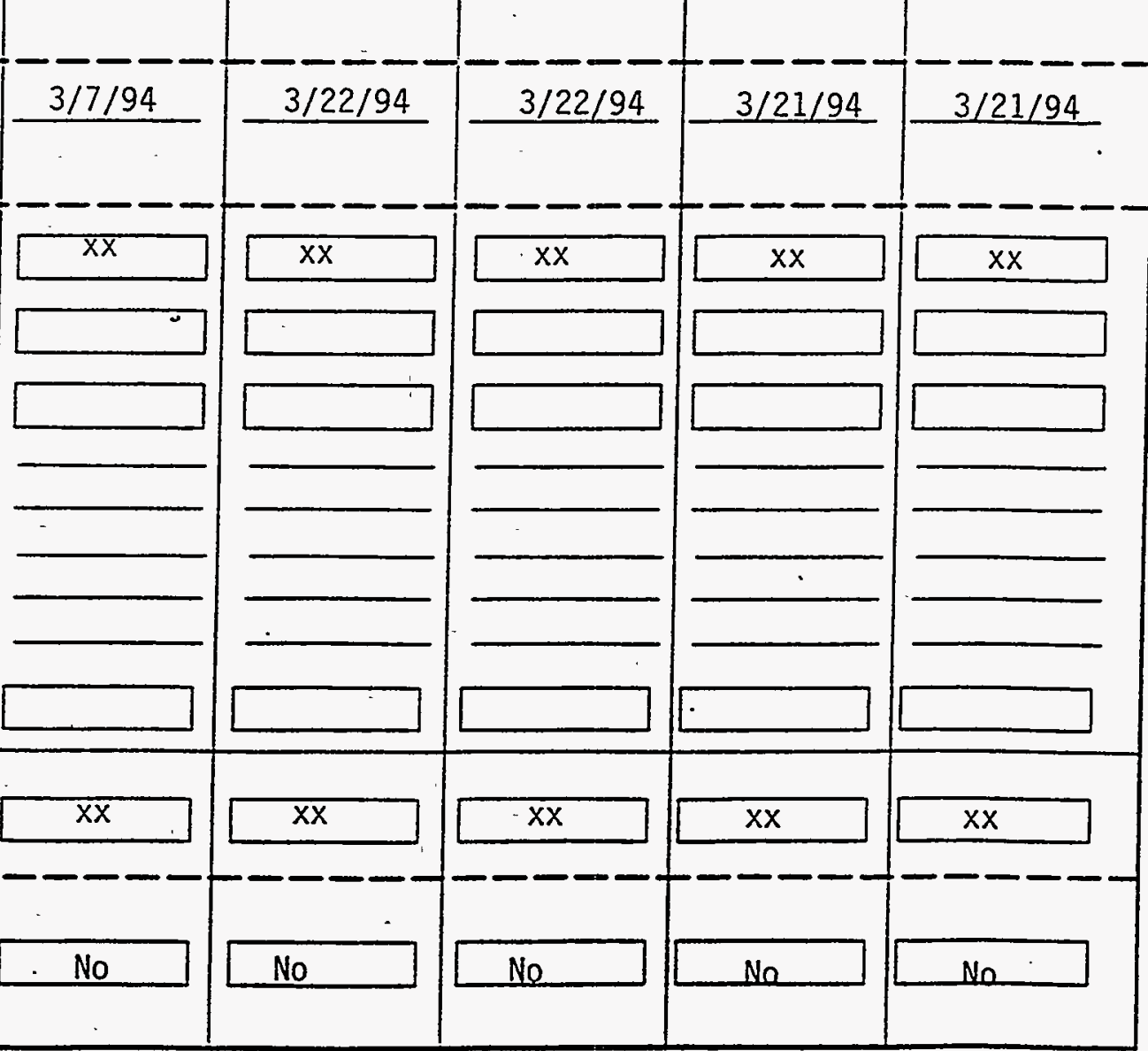

$3 / 21 / 94$ 
rage 7 of 11

$12-13-1$

$23-110-1$

$23-112-1$ $25-3102-3 \quad 25-3205-1$

Tank lentification Number

7. Substance Currently or Last Stored In Greatest Quantity by Volume Gasoline

Diesel

Gasohol

Kerosene

Heating Oil

Used Oil

Other, Please specify

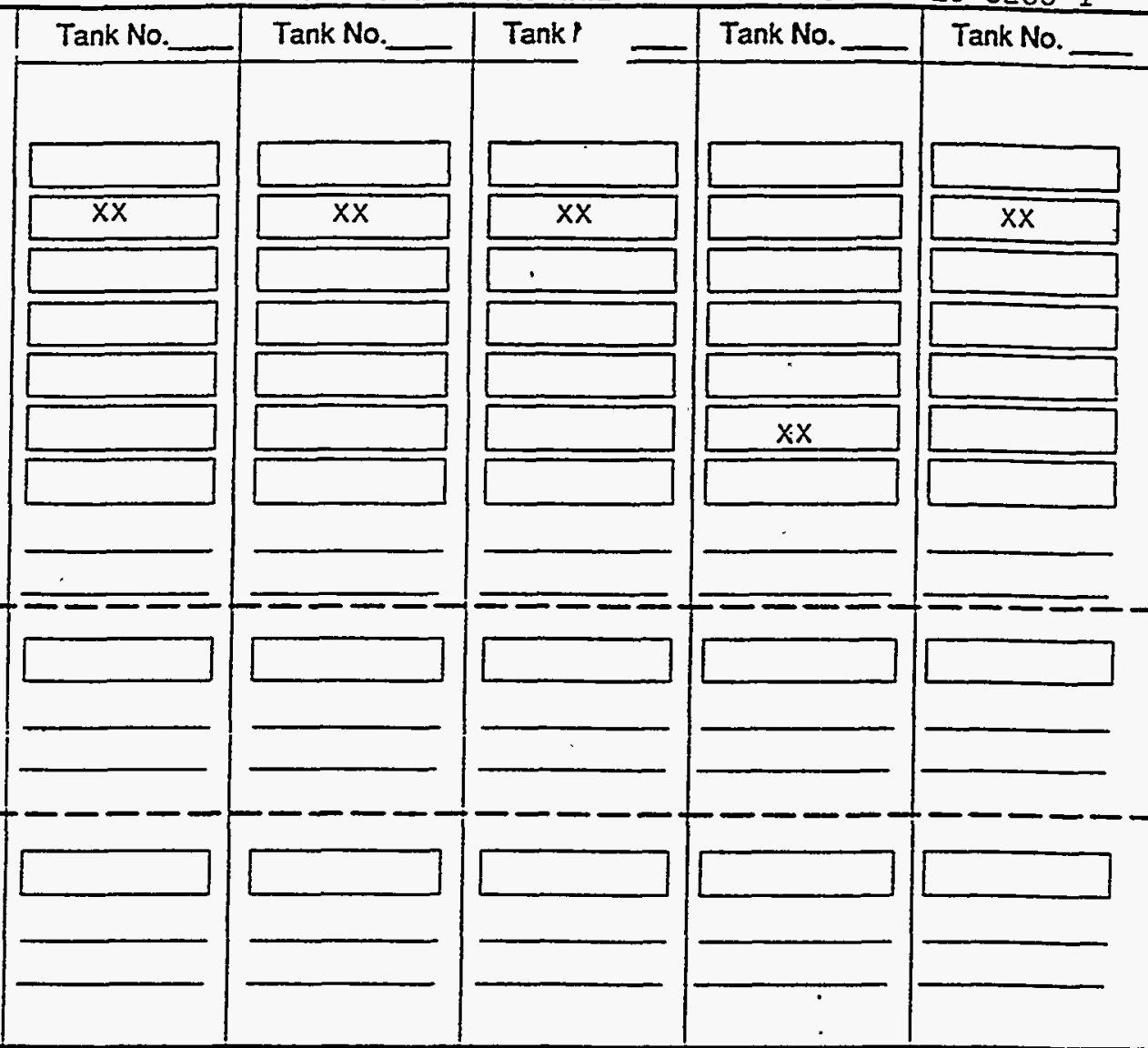

\section{$X$. TANKS OUTT OF USE, OR CHANGE IN SERVICE}

1. Closing of Tank

A. Estimated date last used

Not Determ Not Determ. Not Determ. Not:Determ Not Determ. (mo./day/year)

B. Estimate date tank closed (mo./day/year)

C. Tank was removed from ground

D. Tank was closed in ground

E. Tank filled with inert material Describe

F. Change in service

2. Site Assessment Completed

$\mathrm{xx}$

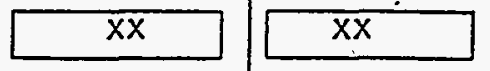

$3 / 9 / 94$ $10 / 7 / 93$

$10 / 20 / 93$

$10 / 6 / 93$
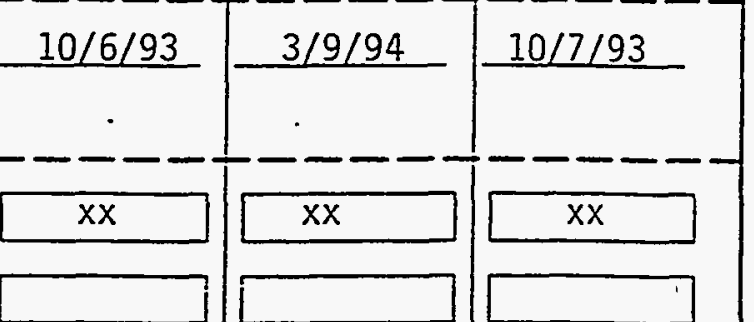

Evidence of a leak detected

Yes No

No

Yes

No 
Page 8 of 11

Tank Identification Number

7. Substance Currently or Last Stored In Greatest Quantity by Volume Gasoline

Diesel

Gasohol

Kerosene

Heating Oil

Used Oil

Other, Please specify

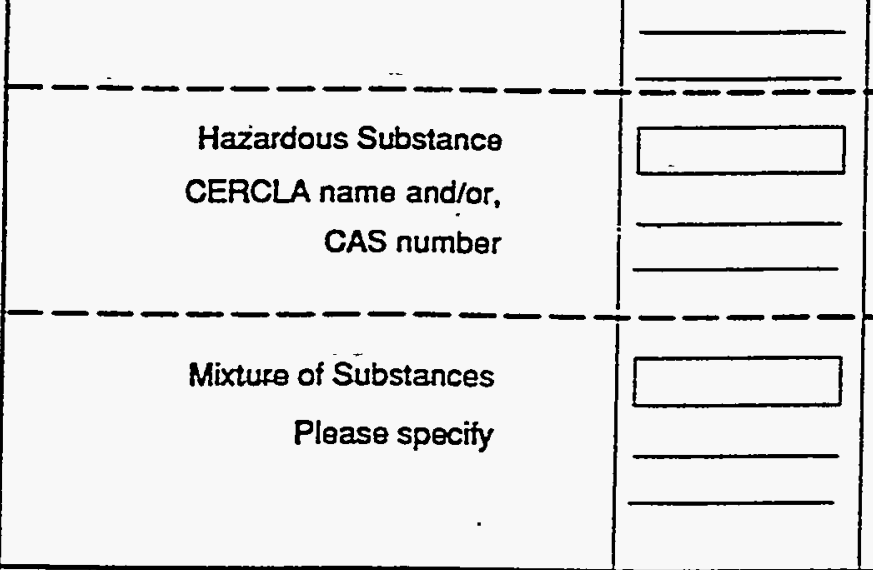

1. Closing of Tank

A. Estimated date last used (mo/dayryear)

B. Estimate date tank closed (mo./day'year)

- - - - - - - - - - - -

C. Tank was removed from ground

D. Tank was closed in ground

E. Tank filled with inert material Describe

F. Change in service

\section{Site Assessment Completed}

Evidence of a leak detected

27-5170-1 27-5310-1

Not Determ. Not. Determ. $\frac{1}{1}$$$
10
$$

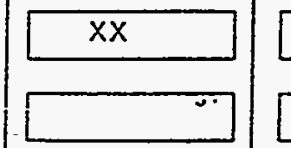

$3 / 17 / 94$
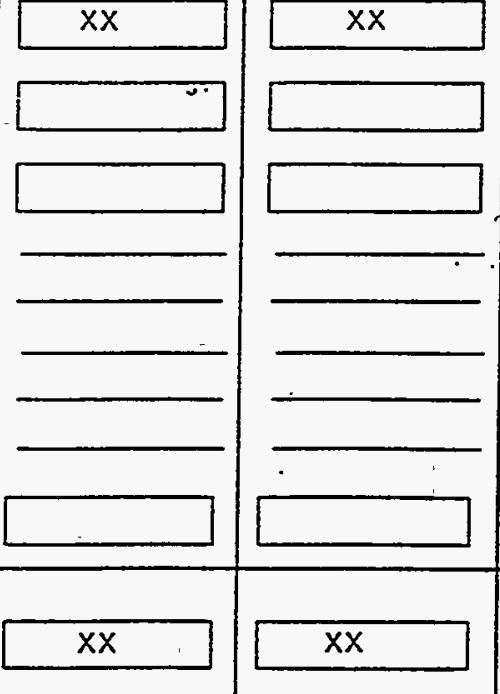

$X$. TANKS OUT OF USE, OR CHANGE IN SERVICE

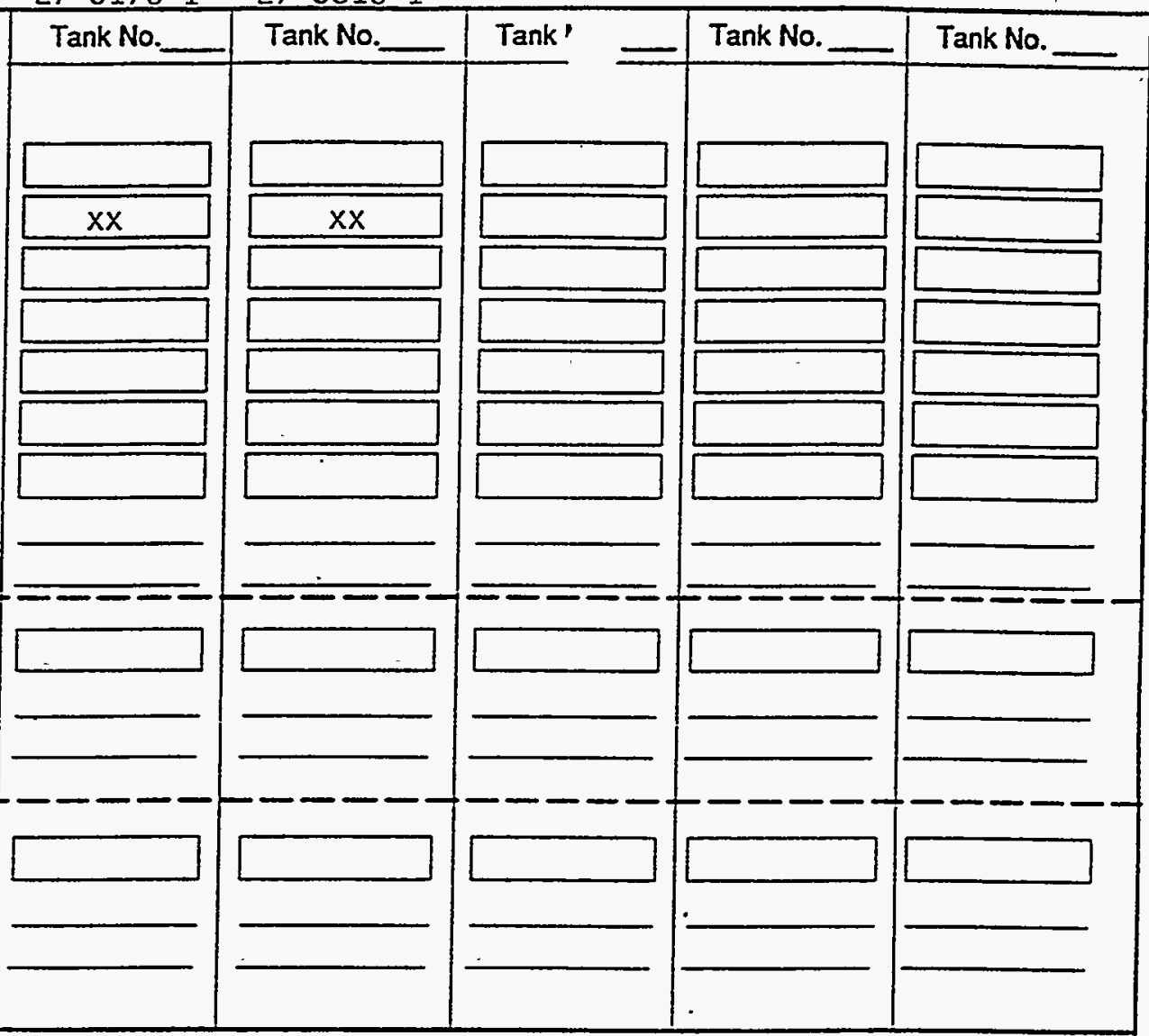

Yes

Yes

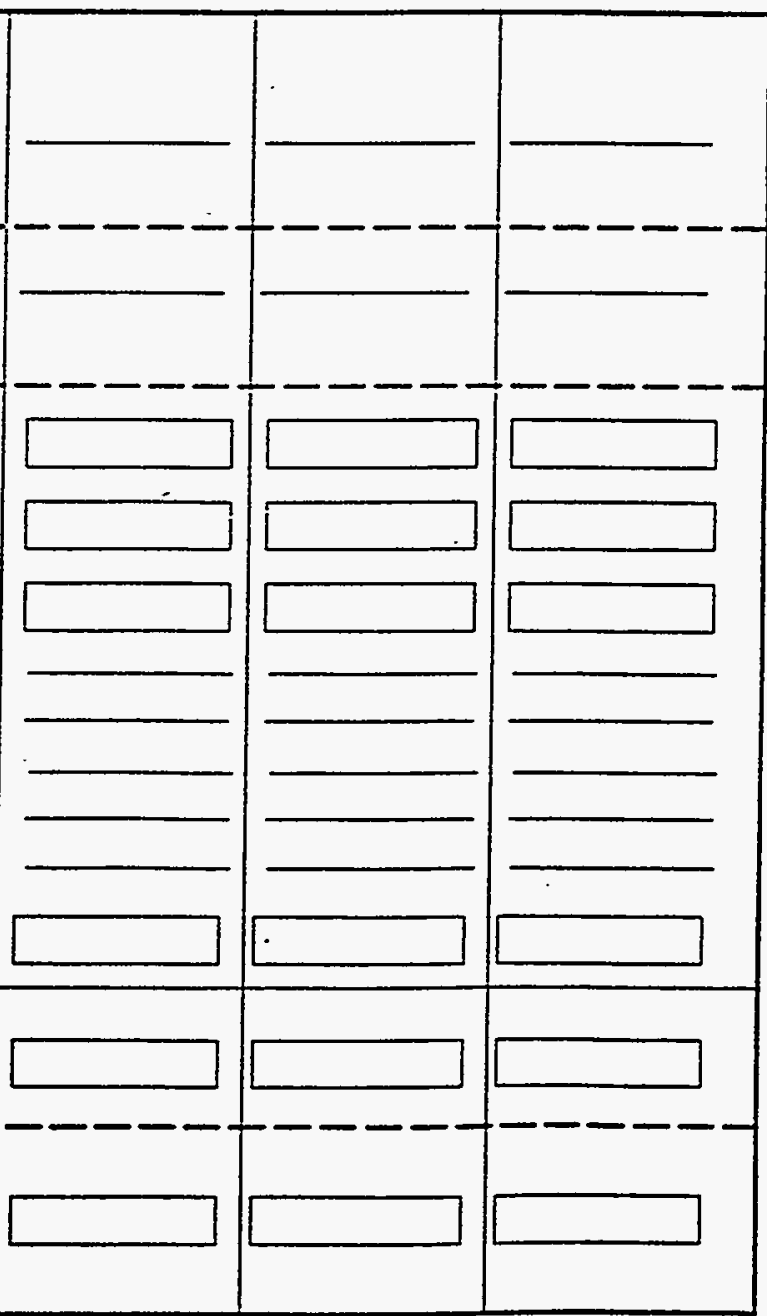

Page 4 
Page 9 of 11

12-8-1 $\quad 12-8-2$ $12-9-1$ 12-9-2

\begin{tabular}{|c|c|c|c|c|c|c|c|c|c|c|}
\hline XI. CEATIFICATION OF COM & NCE (CC & DMPLET & FORA & LNEW/ & ND UPC & JED & & ATTHISL & OCATIO & \\
\hline Tank Identification Number & Tank N & 10. __ & Tank & vo.___ & Tank N & o. - _ & Tank & o. _ - & Tank No & - \\
\hline 1. Installation & $\begin{array}{l}\text { Not } \\
\text { Applic }\end{array}$ & cable & $\begin{array}{l}\text { Not } \\
\text { Appl }\end{array}$ & icable & $\begin{array}{l}\text { Not } \\
\text { App } 1\end{array}$ & icable & $\begin{array}{l}\text { Not } \\
\text { Appl }\end{array}$ & icable & $\begin{array}{l}\text { Not } \\
\text { Appli }\end{array}$ & able \\
\hline $\begin{array}{l}\text { A. Installer cortified by tank and } \\
\text { piping manufacturers }\end{array}$ & & & & & & & & & & \\
\hline $\begin{array}{l}\text { B. Installer certified or licensed by the } \\
\text { implementing agency }\end{array}$ & & & & & & & & & & \\
\hline $\begin{array}{l}\text { C. Installation inspected by a } \\
\text { registered engineer }\end{array}$ & & & & & & & & & & \\
\hline $\begin{array}{l}\text { D. Installation inspected and } \\
\text { approved by implementing agency }\end{array}$ & & & & & & & & & & \\
\hline $\begin{array}{l}\text { E. Manufacturer's installation } \\
\text { chocklists have been completed }\end{array}$ & & & & & & & & & & \\
\hline F. Anotiner method allowec by State & & & & & & & & & & \\
\hline 2. Release Detection (Mark all that apply) & TANK & PIPING & TANK & PIPING & TANK & PIPING & TANK & PIPING & TANK & PIPING \\
\hline A. Manual tank gauging & & & $L$ & & & & & & & \\
\hline B. Tank tightness testing & & & L & & L & & & & & \\
\hline C. Inventory controls & & & L & & & & & & & \\
\hline D. Automatic tank gauging & & & $=$ & & $L$ & & $L$ & & & \\
\hline E. Vapor monitoring & & & L & & L & & L & & & \\
\hline F. Groundwater monitoring & & & L & & L & & L & & & \\
\hline $\begin{array}{l}\text { G. Interstitial monitoring double walled } \\
\text { tank/piping }\end{array}$ & & & L & & & & L & & & \\
\hline $\begin{array}{l}\text { H. Interstitial monitoring/secondary } \\
\text { containment }\end{array}$ & & & L & & & & $\square$ & & & \\
\hline 1. Automatic line leak detectors & 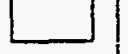 & & L & L & L: & 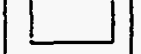 & $\square$ & & L & \\
\hline J. Line tigntness testing & & & & & & & & & & \\
\hline K. Other method allowed by & $\square$ & & L & & L & 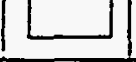 & L & & & \\
\hline & 工 & & & & & & & & & \\
\hline 3. Spill and Overfill Protection & & & & & & & & & & \\
\hline A. Overfill device installed & & & & & & & & & \begin{tabular}{|c} 
\\
\end{tabular} & \\
\hline B. Spill device installied & & & & & & & & & & \\
\hline
\end{tabular}

OATH: I certify the information concerning installation that is provided in section XI is true to the best of my belief and knowledge. Installer: NOT APPLICABLE 
Page 10 of 11

\begin{tabular}{|l}
\hline XI. CERTIFICATION OF COI \\
\hline Tank Identification Number \\
\hline 1. Installation \\
A. Installer certified by tank and \\
piping manufacturers \\
B. Installer certified or licensed by the \\
implementing agency \\
C. Installation inspected by a \\
registered engineer \\
D. Installation inspected and \\
approved by implementing agency \\
E. Manufacturer's installation \\
checklists have been completed \\
F. Anotiner method allowed by State \\
agency. Please specify.
\end{tabular}

2. Release Detection (Mark all that apply)

A. Manual tank gauging

B. Tank tightness testing

C. Inventory controls

D. Automatic tank gauging

E. Vapor monitoring

F. Groundwater monitoring

G. Interstitial monitoring double walled tank/piping

H. Interstitial monitoring/secondary containment

I. Automatic line leak detectors

j. Line tignitness testing

K. Other method allowed by Implementing Agency. Please specify.

\section{Spill and Overfill Protection}

A. Overifl device installed

B. Spill device installed
12-13-1

23-110-1

$23-112-1 \quad 25-3102-3$

25-3205-1

ANCE (COMPLETE FOR ALL NEW AND UPi DED TANKS AT THIS LOCATION)

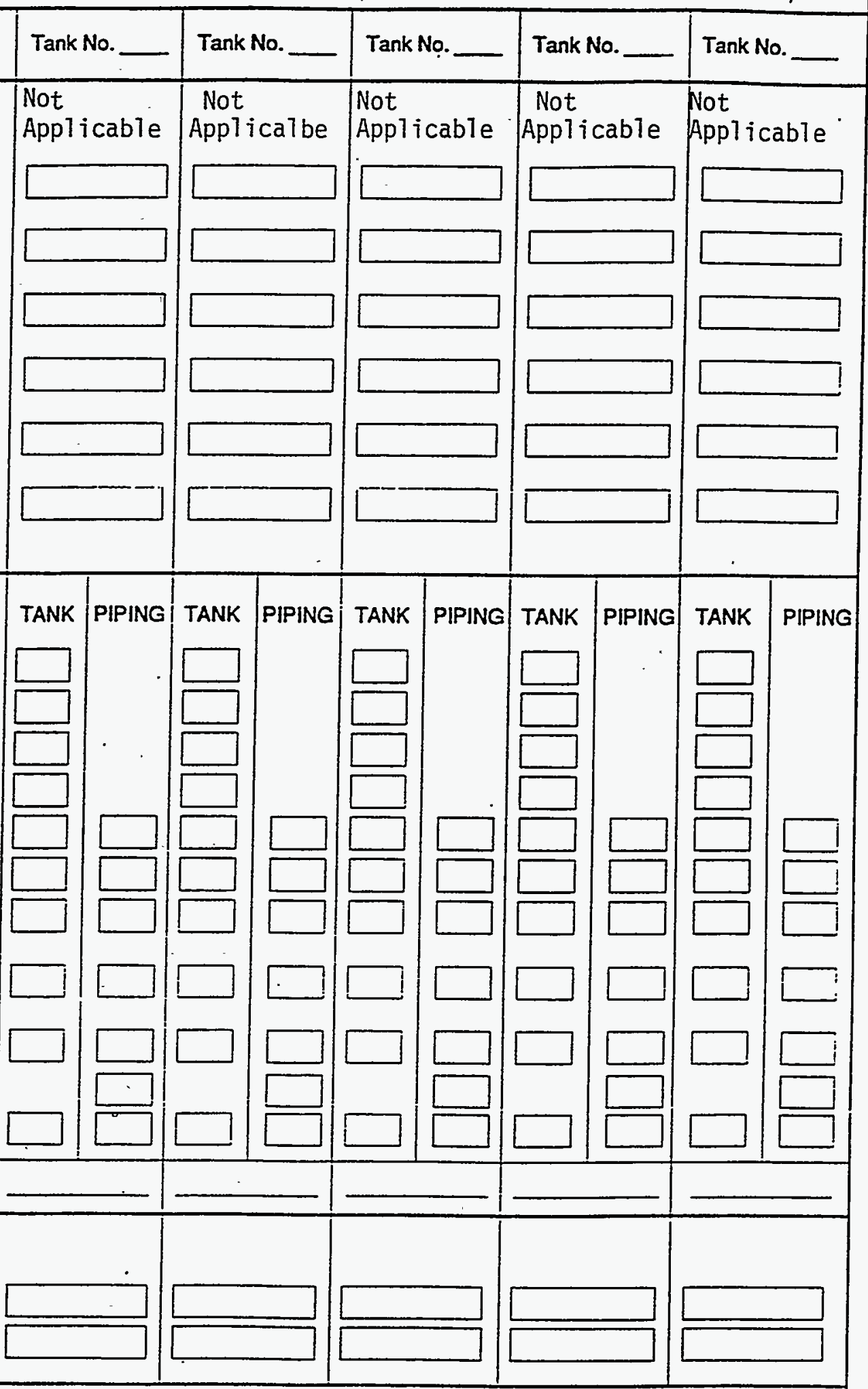

OATH: I certify the information concerning installation that is provided in section XI is true to the best of my belief and knowledge. Installer: $\frac{\text { NOT APPLICABLE }}{\text { Name }}$ 


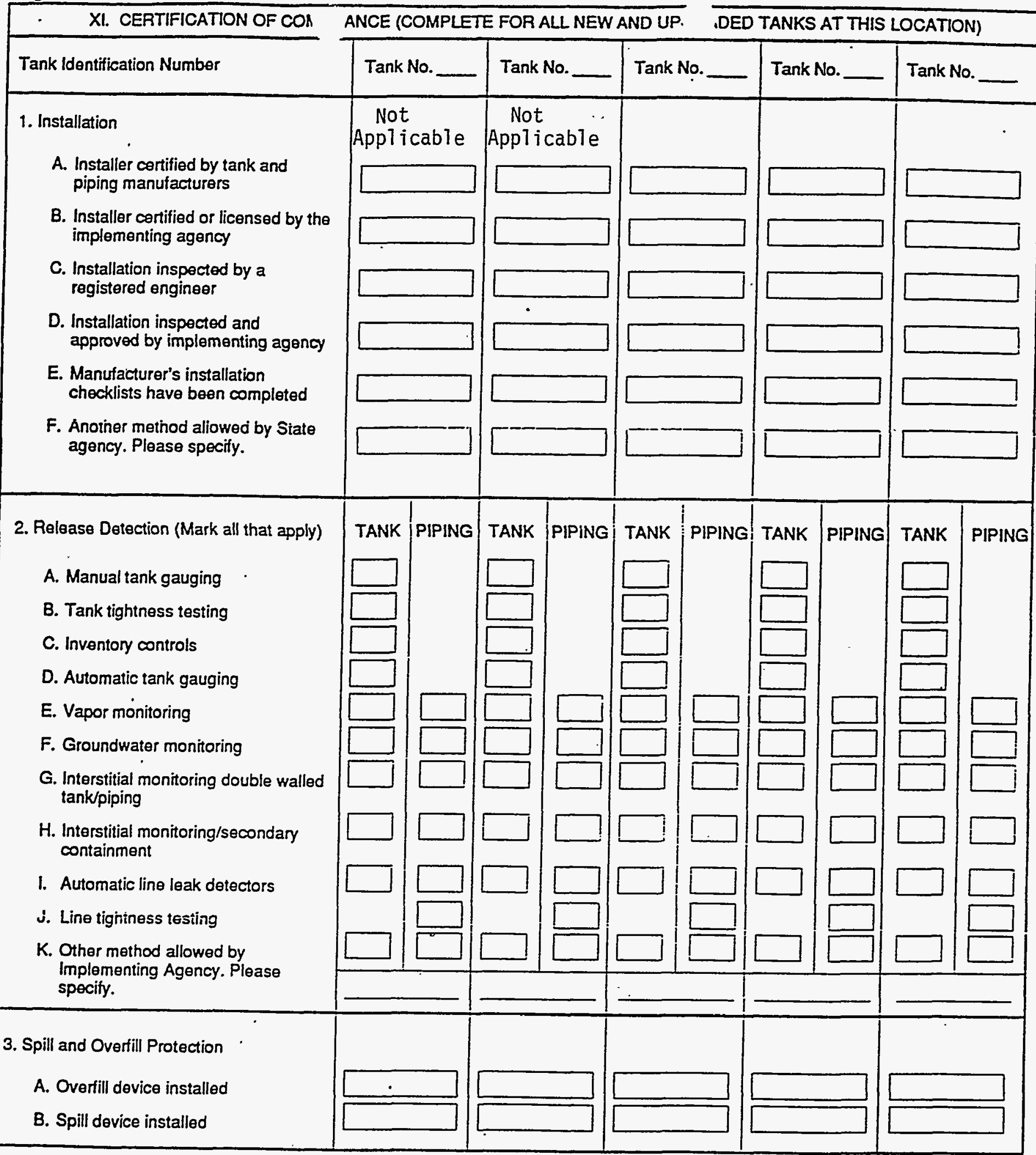

OATH: I certify the information conceming installation that is provided in section XI is true to the best of my belief and knowledge. Installer: NOT APPLICABLE

$\begin{array}{lllll}\text { Name } & \text { Signature } & \text { Date } \\ & \text { Position } & \text { Company }\end{array}$



ENVIRONMENTAL RESTORATION PROGRAM

ABANDONED UNDERGROUND STORAGE TANKS (PHASE 2 - FY93)

SUMMARY OF ANALYTICAL RESULTS FOR SOIL SAMPLES

COLLECTED AT THE BOTTOM ENDS OF

UNDERGROUND STORAGE TANKS 

ENVIRONMENTAL RESTORATION PROGRAM ABANDONED UNDERGROUND STORAGE TANKS (PHASE 2 - FY93):

SUMMARY OF ANALYTICAL RESULTS FOR SOIL SAMPLES COLLECTED AT THE BOTTOM ENDS OF UNDERGROUND STORAGE TANKS

\begin{tabular}{|c|c|c|c|c|c|c|c|c|}
\hline $\begin{array}{l}\text { TANK AND SAMPIE } \\
\text { NUMBER } \\
\qquad y\end{array}$ & \multicolumn{2}{|c|}{$\begin{array}{c}\mathrm{TPH}^{1} \\
(\mathrm{mg} / \mathrm{kg})\end{array}$} & \multirow{2}{*}{$\begin{array}{c}\begin{array}{c}\mathrm{PCBs}^{2} \\
(\mathrm{mg} / \mathrm{kg}= \\
\mathrm{ppm})\end{array} \\
N^{*}\end{array}$} & \multicolumn{2}{|l|}{$\begin{array}{l}\operatorname{vocs^{3}}: \\
(u g / \mathrm{kg})\end{array}$} & \multirow{2}{*}{$\frac{\begin{array}{c}\text { Semi -VOCs } \\
(u g / k g)\end{array}}{N A}$} & \multicolumn{2}{|c|}{$\underset{(\mathrm{mg} / \mathrm{T})}{\text { TCLP METALS }}$} \\
\hline $\begin{array}{l}\text { Tank: } \\
\text { Sample: } 1111-1-1\end{array}$ & & $\begin{array}{r}<10.0 \\
<5.0 \\
<10.0 \\
\end{array}$ & & $\begin{array}{l}\text { Methylene Chloride } \\
\text { Xylenes }\end{array}$ & $\frac{79 *}{7}$ & & $\begin{array}{l}\text { Barium. } \\
\text { Silver }\end{array}$ & $\begin{array}{l}0.49 \\
0.05\end{array}$ \\
\hline $\begin{array}{l}\text { Tank: } 11-1-1 \\
\text { Sampie: } 1111 W\end{array}$ & & $\begin{array}{r}<10.0 \\
<5.0 \\
13.4\end{array}$ & NA & $\begin{array}{l}\text { Methylene, Chloride } \\
\text { Toluene } \\
\text { Ethylbenzene } \\
\text { Xylenes } \\
\end{array}$ & $\begin{array}{l}52^{*} \\
25 \\
5 \\
41 \\
\end{array}$ & NA & $\begin{array}{l}\text { Barium } \\
\text { Chromium } \\
\text { Silver }\end{array}$ & $\begin{aligned} & 0.44 \\
< & 0.016 \\
< & 0.033\end{aligned}$ \\
\hline $\begin{array}{ll}\text { Tank: } & 12-8-1 \\
\text { Sample: } & 12-8-1-11.8 S\end{array}$ & $\begin{array}{l}D \\
G \\
0 \\
\end{array}$ & $\begin{array}{l}<10.0 \\
<5.0 \\
<10.0 \\
\end{array}$ & $<0.05$ & Methylene Chloride & $2.8^{*}$ & $N D^{10}$ & Barium & 0.12 \\
\hline $\begin{array}{ll}\text { Tank: } & 12-8-1 \\
\text { Sample: } & 12-8-1-11.6 \mathrm{~N}\end{array}$ & $\begin{array}{l}D \\
G \\
0 \\
\end{array}$ & $\begin{array}{l}<10.0 \\
<5.0 \\
<10.0 \\
\end{array}$ & $<0.05$ & $\begin{array}{l}\text { Methylene Chloride } \\
\text { Acetone }\end{array}$ & $\frac{2.9 *}{15^{* *}}$ & ND & $\begin{array}{l}\text { Barium } \\
\text { Chromium }\end{array}$ & $\begin{array}{r}0.039 \\
<0.031\end{array}$ \\
\hline $\begin{array}{ll}\text { Tank: } & 12-8-2 \\
\text { Sample: } & 12-8-2-12.0 \mathrm{~N}\end{array}$ & $\begin{array}{l}D \\
G \\
0 \\
\end{array}$ & $\begin{array}{r}<10.0 \\
<5.0 \\
<10.0 \\
\end{array}$ & $<0.05$ & Methylene Chloride & $3.1^{*}$ & ND & Barium & 0.14 \\
\hline $\begin{array}{ll}\text { Tank: } & 12-8-2 \\
\text { Sample: } & 12-8-2-13.0 \mathrm{~S}\end{array}$ & $\begin{array}{l}D \\
G \\
0 \\
\end{array}$ & $\begin{array}{r}96 \\
<5.0 \\
<10.0 \\
\end{array}$ & $<0.05$ & $\begin{array}{l}\text { Methylene Chloride } \\
\text { Acetone }\end{array}$ & $\frac{2.5^{*}}{16^{* *}}$ & ND & $\begin{array}{l}\text { Barium } \\
\text { Chromium } \\
\text { Lead }\end{array}$ & $\begin{array}{ll} & 0.11 \\
< & 0.031 \\
< & 0.21 \\
\end{array}$ \\
\hline $\begin{aligned} & \text { Tank: } 12-9-1 \\
& \text { Sample: } 12-9-1-9.2 E \\
& \text { and } 12-9-1-0.2 E \\
&\end{aligned}$ & $\begin{array}{l}D \\
G \\
0 \\
\end{array}$ & $\begin{array}{r}<10.0 \\
<5.0 \\
<10.0 \\
\end{array}$ & $<0.167$ & Acetone & $13 *$ & ND. & Barium & 0.097 \\
\hline $\begin{array}{ll}\text { Tank: } & 12-9-1 \\
\text { Sample: } & 12-9-1-13 \mathrm{~W}\end{array}$ & & $\begin{array}{l}<10.0 \\
<5.0 \\
<10.0 \\
\end{array}$ & $<0.00167$ & Acetone & $28 *$ & ND & Barium & 0.079 \\
\hline $\begin{array}{ll}\text { Tank: } & 12-9-2 \\
\text { Sample: } & 12-9-2-12 E\end{array}$ & & $\begin{array}{l}<10.0 \\
<5.0 \\
<10.0 \\
\end{array}$ & $<0.167$ & $\begin{array}{l}\text { Methylene Chloride } \\
\text { Acetone }\end{array}$ & $\begin{aligned} 5 * \\
13^{*}\end{aligned}$ & ND & $\begin{array}{l}\text { Barium } \\
\text { Chromium }\end{array}$ & $\begin{array}{r}0.090 \\
<0.031\end{array}$ \\
\hline
\end{tabular}




\begin{tabular}{|c|c|c|c|c|c|c|c|}
\hline $\begin{array}{l}\text { TANK AND SAMPLE } \\
\text { NUMBEER }\end{array}$ & $\begin{array}{c}\mathrm{TPH}^{1} \\
(\mathrm{mg} / \mathrm{kg})\end{array}$ & $\begin{array}{l}\mathrm{PCBs}^{2} \\
(\mathrm{mg} / \mathrm{kg}= \\
\mathrm{ppm})\end{array}$ & $\begin{array}{l}\text { VOCs }{ }^{3} \\
(u g / k g\end{array}$ & & $\begin{array}{c}\text { Semi - VOCs }{ }^{4} \\
(u g / k g)\end{array}$ & \multicolumn{2}{|c|}{$\underset{(\mathrm{mg} / 1)}{\text { TCLP METALS }}$} \\
\hline $\begin{array}{ll}\text { Tank: } & 12-9-2 \\
\text { Sample: } & 12-9-2-14 \mathrm{~W}\end{array}$ & 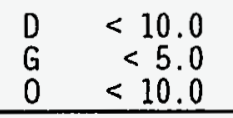 & $\therefore 0.00166$ & Acetone & $21^{*}$ & ND & $\begin{array}{l}\text { Barium } \\
\text { Chromium }\end{array}$ & $\begin{array}{r}0.081 \\
<0.031\end{array}$ \\
\hline $\begin{array}{ll}\text { Tank: } & 12-13-1 \\
\text { Sample: } & \text { ERUSTP2/ } \\
& 1213 \mathrm{~W} \\
\end{array}$ & $D<10.0$ & NA & NA & & $N A$ & \multicolumn{2}{|c|}{ NA } \\
\hline $\begin{array}{ll}\text { Tank: } & 12-13-1 \\
\text { Sample: } & \begin{array}{l}\text { ERUSTP2 I } \\
\text { 1213E }\end{array} \\
\end{array}$ & $0 \quad 116$ & NA & NA & & NA & \multicolumn{2}{|c|}{ NA } \\
\hline $\begin{array}{ll}\text { Tank: } & 23-110-1 \\
\text { Sampie: } & \text { ER-USTPH2/ } \\
& 110 S \\
\end{array}$ & $\begin{array}{l}0 \\
\text { (see analysts } \\
\text { note) }\end{array}$ & NA & NA & & $N A$ & \multicolumn{2}{|c|}{ NA } \\
\hline $\begin{array}{ll}\text { Tank: } & 23-110-1 \\
\text { Sample: } & \text { ER-USTPH2/ } \\
& 110 N \\
\end{array}$ & $\begin{array}{l}0 \\
\text { (see analysts } \\
\text { note) }\end{array}$ & NA & NA & & NA & \multicolumn{2}{|c|}{ NA } \\
\hline $\begin{array}{ll}\text { Tank: } & 23-112-1 \\
\text { Sample: } & \text { ER-USTPH2I } \\
& 112-N\end{array}$ & $\begin{array}{l}0 \\
\text { (see anaiysts } \\
\text { note) }\end{array}$ & NA & NA & & $N A$ & \multicolumn{2}{|c|}{$N A$} \\
\hline $\begin{array}{ll}\text { Tank: } & 23-112-1 \\
\text { Sample: } & \text { ER-USTPH2I } \\
& 112-S \\
\end{array}$ & $\begin{array}{l}0 \quad 45.5 \\
\text { (see analysts } \\
\text { note) }\end{array}$ & NA & NA & & NA & \multicolumn{2}{|c|}{ NA } \\
\hline $\begin{array}{ll}\text { Tank: } & 25-3102-3 \\
\text { Sampie: } & 253102 / \mathrm{S}\end{array}$ & $\begin{array}{l}D \\
D\end{array} \quad \begin{array}{r}22.8 \\
0\end{array}$ & $\therefore 0.167$ & NA & & NA & $\begin{array}{l}\text { Barrum } \\
\text { Chromium } \\
\text { Silver } \\
\end{array}$ & $\begin{array}{ll} & 0.81 \\
< & 0.016 \\
< & 0.033 \\
\end{array}$ \\
\hline $\begin{array}{ll}\text { Tank: } & 25-3102-3 \\
\text { Sample: } & 253102 / N\end{array}$ & $\begin{array}{ll}D & 2090 \\
0 & <10.0\end{array}$ & $<0.167$ & NA & & NA & $\begin{array}{l}\text { Barium } \\
\text { Chromium } \\
\text { Silver } \\
\end{array}$ & $\begin{array}{ll} & 1.0 \\
< & 0.016 \\
< & 0.033 \\
\end{array}$ \\
\hline $\begin{array}{ll}\text { Tank: } & 25-3205-1 \\
\text { Sample: } & \text { ER-USTPH2I } \\
& 3205-S-R\end{array}$ & $D<10.0$ & NA & NA & & NA & \multicolumn{2}{|c|}{ NA } \\
\hline
\end{tabular}




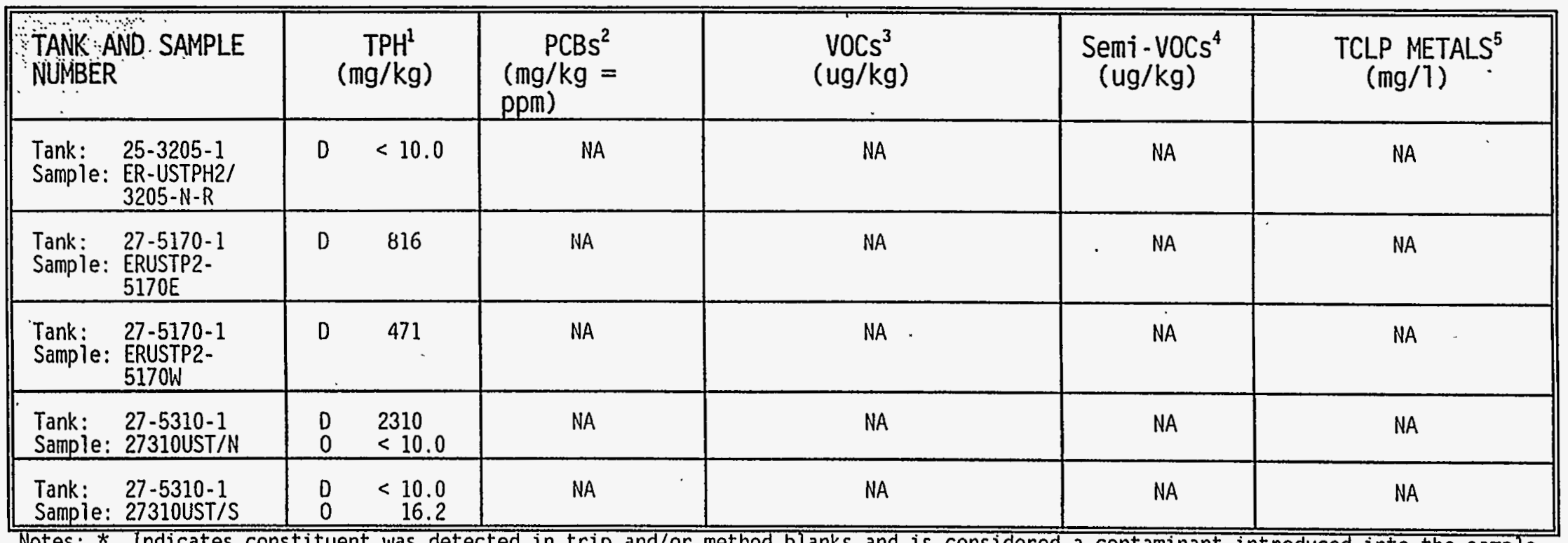

Notes: * Indicates constituent was detected in trip and/or method blanks and is considered a contaminant introduced into the sample either during sampling, transport. storage. and/or analysis

** Indicates acetone is a suspected contaminant introduced into the sample during sampling, transport. and/or analysis since acetone was not detected in the analyses of the tank contents (was not detected in the trip or method blanks either)

1 Total Petroleum Hydrocarbons (TPH): EPA Method 8015. Modified

2 Polychlorinated Biphenyis (PCB's): EPA Method 8080

3 Volatile Organic Compounds (VOC's): EPA Method 8240 or 8260

4 Semi-Volatile Organic Compounds (Semi-VOC's): EPA Method 8270

5 Toxicity Characteristic Leaching Procedure (TCLP) Metals: EPA Extraction Method 1311 and Method 6010

6 TPH as Diese? (D)

7 TPH as Gasoline (G)

8 TPH as Oil (0)

9 Not Analyzed (NA)

10 Not detected above anaiytical method/laboratory detection level 

ANALYTICAL RESULTS FOR TANK NUMBERS

12-13-1 AND 27-5310-1 



\section{MEMORANDUM}

To

S. J. Nacht

From

G. A. Clark

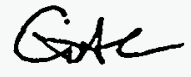

Date

November 17, 1993

Subject

SAMPLE ANALYTICAL RESULTS

Enclosed are Analytical Services Department's results, requested by T. A. Wilczek, for the total petroleum hydrocarbons (gasoline/diesel/0il) analyses of four soil and two water samples collected on October 20, 1993, in Areas 12 and 27. Also enclosed are the results for the gross gamma analysis of these samples.

Please direct any questions you may have about the nonradioanalytical results to Roger Mitchell (295-7220) or Jerry Dugas (295-7997), and questions about the radioanalytical results to Al Latham (295-7472).

GAC:RNL1277:rn

Enclosures

As stated

cy: Central Files, w/o encls.

A. R. Latham, w/o encls.

L. S. Sygitowicz, w/o encls.

T. A. Hilczek, w/encls:

ACS Packet No. 93-10-101, w/o encls. 
REECO

BOB DODGE

ENVIRONMENTAL RESTORATIOH

TECHMOLOGY DEVELOPHENT

AtTn: TOH HILCZEK

Purchase Order: $3754-022$

Invoice Number:

REPORT TO TOH WILCZEK, ERTD

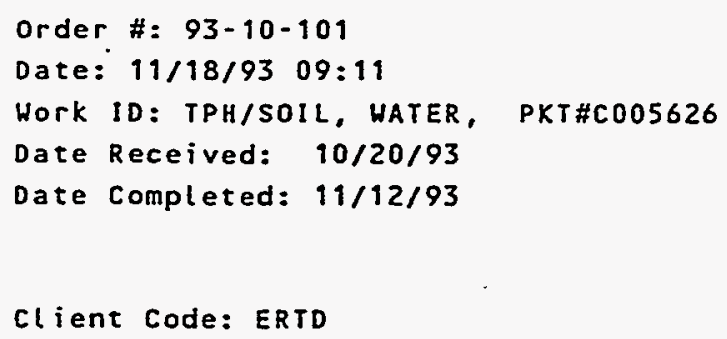

\section{SAMPLE IDENT IFICATIOH}

\begin{tabular}{|c|c|}
\hline $\begin{array}{l}\text { Sample } \\
\text { Number }\end{array}$ & $\begin{array}{c}\text { Sample } \\
\text { Description }\end{array}$ \\
\hline 01 & ERUSTP2/5170-E \\
\hline 02 & ERUSTP2/5170-H \\
\hline 03 & ERUSTP $2 / 5170-81 \mathrm{HSE}$ \\
\hline 04 & ERUSTP2/1213-H \\
\hline 05 & ERUSTP2/1213-E \\
\hline 06 & ERUSTP2/1213-RINSE \\
\hline 07 & $93-10-101-0 C 1$ \\
\hline
\end{tabular}
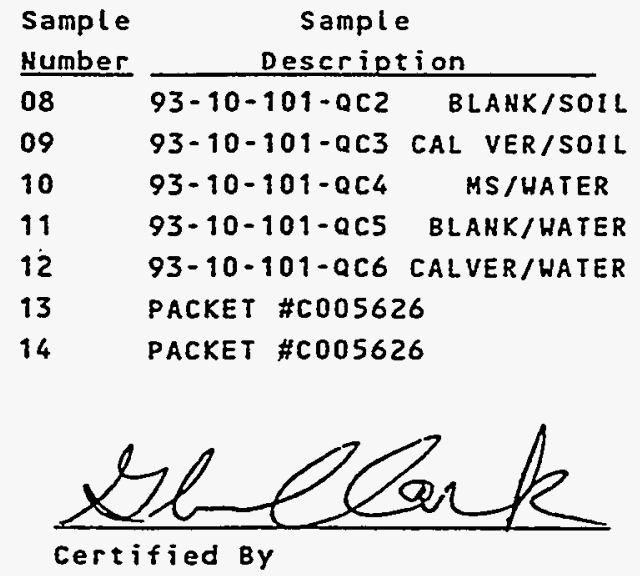
RADIOISOTOPE SCREEH

Method:

Samp Sample Description

13A PACKET \#C005626

RADIOKUCLIDE FIHAL REPORT

Hethod:

Samp Sample Description

14 A PACKET \#C005626

TPH - DIESEL

Hethod: GC

\begin{tabular}{|c|c|}
\hline $01 \mathrm{~A}$ & ERUSTP2/5170-E \\
\hline $02 A$ & ERUSTP2/5170-H \\
\hline $03 \mathrm{~A}$ & ERUSTP2/5170-RINSE் \\
\hline $04 \mathrm{~A}$ & ERUSTP2/1213-H \\
\hline 05A & ERUSTP2/1213-E \\
\hline D6A & ERUSTP2/1213-RIHSE \\
\hline $07 A$ & $93-10-101-0 C 1$ \\
\hline OBA & $93-10-101-0<2$ \\
\hline 09A & 93-10-101-QC3 CAL VER/SOIL \\
\hline $10 \mathrm{~A}$ & $93-10-101-0.44$ \\
\hline $11 \mathrm{~A}$ & 93-10-101-QC5 BLAHK/WATER \\
\hline $12 A$ & 93-10-101-QC6 CALVER/HATER \\
\hline
\end{tabular}

TPH - GASOLIHE

Method: GC

\begin{tabular}{|c|c|c|}
\hline $01 A$ & \multicolumn{2}{|c|}{ ERUSTP2/5170-E } \\
\hline $02 A$ & \multicolumn{2}{|l|}{ ERUSTP2/5170-W } \\
\hline $03 A$ & \multicolumn{2}{|c|}{ ERUSTP $2 / 5170-R I N S E$} \\
\hline $04 \mathrm{~A}$ & \multicolumn{2}{|l|}{ ERUSTP2/1213-H } \\
\hline $05 A$ & \multicolumn{2}{|l|}{ ERUSTP2/1213-E } \\
\hline $06 A$ & \multicolumn{2}{|c|}{ ERUSTP2/1213-RINSE } \\
\hline $07 \mathrm{~A}$ & $93-10-101-\mathrm{QCl}$ & MS/SOIL \\
\hline $08 \mathrm{~A}$ & $93-10-101-0<2$ & BLANK/SOIL \\
\hline 09A & $93-10-101-0<3$ & CAL VER/SOIL \\
\hline $10 \mathrm{~A}$ & $93-10-101-0,4$ & MS/WATER \\
\hline $11 \mathrm{~A}$ & $93-10-101-0,5$ & BLANK/WATER \\
\hline $12 A$ & $93-10-101-0,6$ & CALVER/WATER \\
\hline
\end{tabular}

Minimum: Haximum:

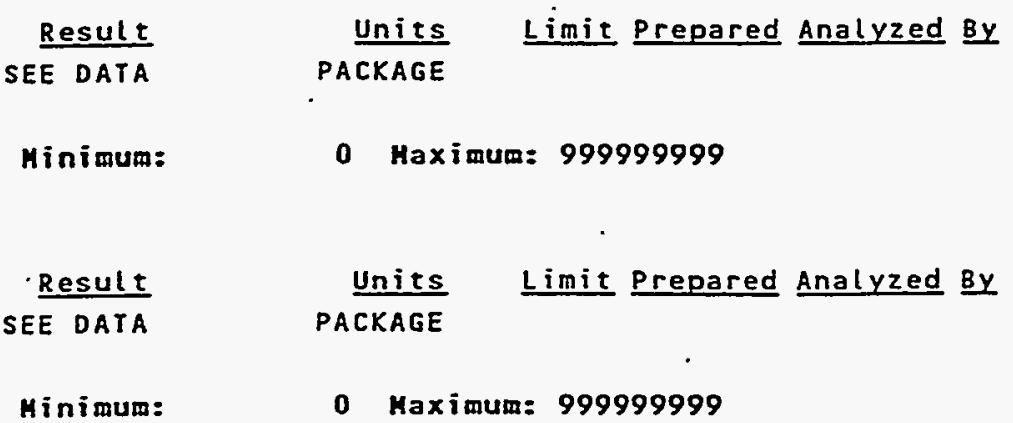

$\begin{array}{rrrr}\frac{\text { Result }}{816} & \frac{\text { Units }}{\mathrm{mg} / \mathrm{Kg}} & \text { Limit Prepared } & \frac{\text { Analyzed }}{11 / 04 / 93} \frac{\mathrm{BY}}{\mathrm{TJR}} \\ 471 & \mathrm{mg} / \mathrm{Kg} & 11 / 04 / 93 \mathrm{TJR} \\ 2.20 & \mathrm{mg} / \mathrm{L} & 11 / 03 / 93 \mathrm{TJR} \\ <10.0 & \mathrm{mg} / \mathrm{Kg} & 11 / 04 / 93 \mathrm{TJR} \\ 116 & \mathrm{mg} / \mathrm{Kg} & 11 / 04 / 93 \mathrm{TJR} \\ 0.730 & \mathrm{mg} / \mathrm{L} & 11 / 03 / 93 \mathrm{TJR} \\ 92 & \% \text { RECOVERY } & 11 / 04 / 93 \mathrm{TJR} \\ <10.0 & \text { mg/Kg } & 11 / 04 / 93 \mathrm{TJR} \\ 110 & \text { \% RECOVERY } & 11 / 04 / 93 \mathrm{TJR} \\ \text { NA } & \text { \% RECOVERY } & & \mathrm{TJR} \\ 0.5 & \text { mg/L } & 11 / 03 / 93 \mathrm{TJR} \\ 76 & \text { \% RECOVERY } & 11 / 03 / 93 \mathrm{TJR}\end{array}$

Hinimum:

O Maximum: 999999999

\begin{tabular}{|c|c|c|c|}
\hline Result & Units & Limit Prepared Analyzed & $\underline{B y}$ \\
\hline$<5.0$ & $\mathrm{mg} / \mathrm{kg}$ & $11 / 02 / 93$ & $T J R$ \\
\hline$<5.0$ & $\mathrm{mg} / \mathrm{Kg}$ & $11 / 02 / 93$ & $T J R$ \\
\hline$<5.0$ & $\mathrm{mg} / \mathrm{l}$ & $11 / 02 / 93$ & T J R \\
\hline$<5.0$ & $\mathrm{mg} / \mathrm{kg}$ & $11 / 02 / 93$ & T JR \\
\hline$<5.0$ & $\mathrm{mg} / \mathrm{kg}$ & $11 / 02 / 93$ & TJR \\
\hline$<5.0$ & $\mathrm{mg} / \mathrm{L}$ & $11 / 02 / 93$ & $T J R$ \\
\hline 171 & \% RECOVERY & $11 / 02 / 93$ & T JR \\
\hline$<5.0$ & $\mathrm{mg} / \mathrm{kg}$ & $11 / 02 / 93$ & T JR \\
\hline 101 & \% RECOVERY & $11 / 02 / 93$ & $T J R$ \\
\hline 119 & \% RECOVERY & $11 / 02 / 93$ & T J R \\
\hline$<5.0$ & $\mathrm{mg} / \mathrm{L}$ & $11 / 02 / 93$ & T JR \\
\hline 101 & \% RECOVERY & $11 / 02 / 93$ & T J R \\
\hline
\end{tabular}


Order 93-10-109

11/18/93 09:11
REECO ASD/ACS

REGULAR TEST RESULTS BY TEST
TPH - OIL

Method: GC

Samp Sample Description

01A ERUSTP2/5170-E

O2A ERUSTP2/5170-H

O3A ERUSTP2/5170-RINSE

04A ERUSTP2/1213-H

O5A ERUSTP2/1213-E

O6A ERUSTP2/1213-RINSE

07A 93-10-101-QC1 MS/SOIL

08A 93-10-101-OC2 BLAHK/SOIL

O9A 93-10-101-QC3 CAL VER/SOIL

10A 93-10-101-QC4 . HS/WATER

11A 93-10-101-0C5 BLANK/HATER

$12 A$ 93-10-101-QC6 CALVER/HATER

TPH - TOTAL

Hethod: GC

Samp Sample Description

OIA ÉRUSTP2/5170-E.

02A ERUSTP2/5170-H

03A ERUSTP2/5170-RINSE

04A ERUSTP2/1213-W

05A ERUSTP2/1213-E

06A ERUSTP2/1213-RINSE

08A 93-10-101-QC2 BLANK/SOIL

11A 93-10-101-QC5 BLANK/WATER
Hinimum:

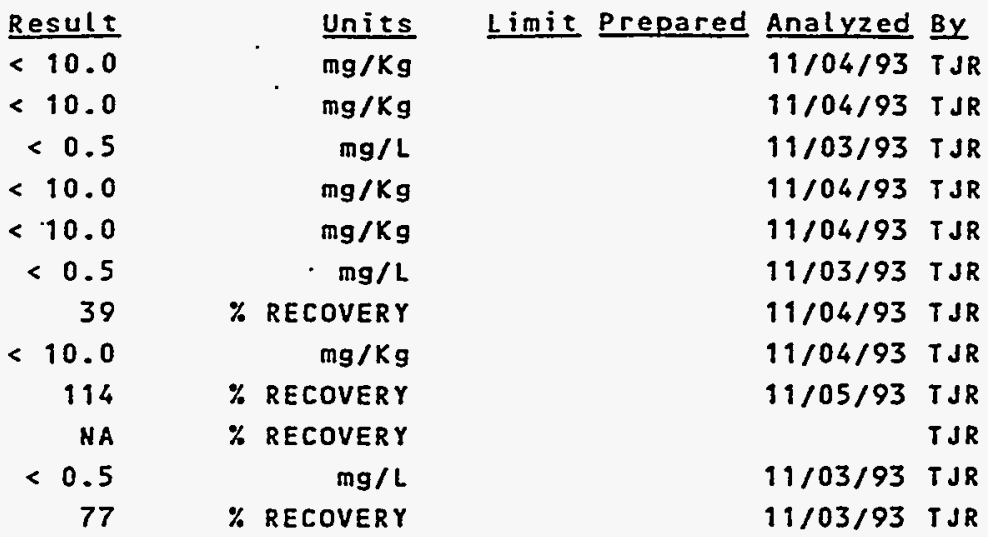

Minimum:

- Maximum: 999999999

Result

816

471

2.20

$<25.0$

116

0.730

$<25.0$

$<6.0$

O Maximum: 999999999

$11 / 03 / 93$ TJR

Units Limit Prepared Analyzed By $\mathrm{mg} / \mathrm{Kg}$

$\mathrm{mg} / \mathrm{Kg}$

$\mathrm{mg} / \mathrm{L}$

$\mathrm{mg} / \mathrm{Kg}$

$\mathrm{mg} / \mathrm{Kg}$

$\mathrm{mg} / \mathrm{L}$

$\mathrm{mg} / \mathrm{Kg}$

$\mathrm{mg} / \mathrm{L}$ 
TPH Analyst's Notes:

All analyses of the sample extracts, with the exception of ERUSTP2/1213-W, produced chromatograms indicating the presence of a component whose reponse maximum $l i e s$ between that seen for the $D i e s e l$ and $0 i l$ standards. However, as the geometry of the chromatogram is similar to that of Diesel, the Diesel calibration was used to quantitate the component. The reported values should be viewed as rough estimates of the contaminant present.

Analysis of the aqueous extracts was performed on the 3rd of November. The analyses of the Diesel and Dil Calibration Verification standards on that column returned at approximately $75 \%$ of their expected values. The results for those samples were not adjusted.

The aqueous biesel and 0 il matrix spikes were extracted from samples of other work orders which were highly contaminated and therefore did not allow for the recovery of the spikes.

The soil oil matrix spike was prepared from a sample of another set which was highly contaminated with Diesel and $0 i l$. The spike could not be seen due to these levels. 


\section{Reynolds Electrical \& Engineering Co., Inc.}

SAMPLE CHAIN-OF-CUSTODY RECORD

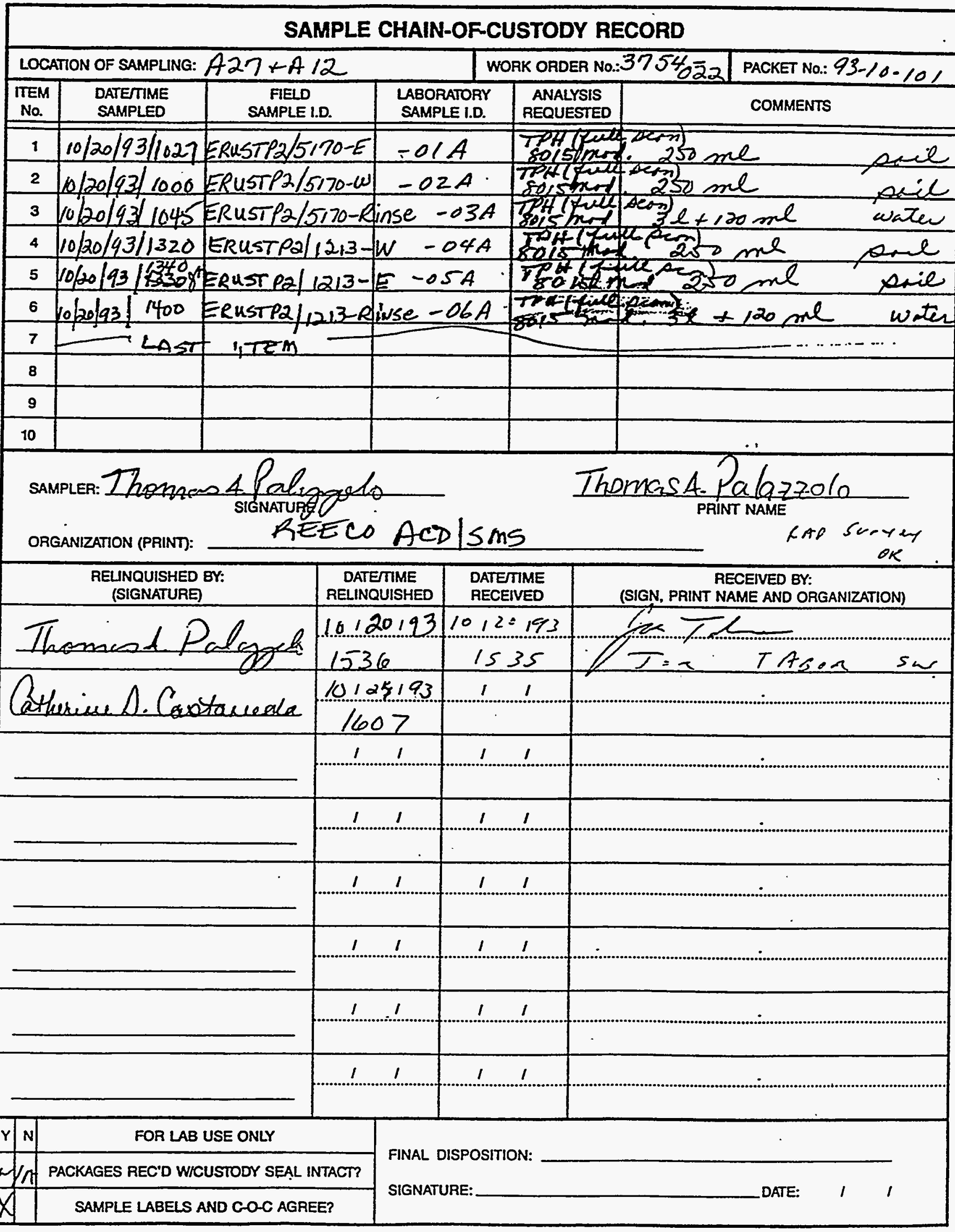




\section{APPENDIX B}

\section{LABORATORY ANALYTICAL DATA PACKETS}





\section{Reynolds Electrical \& Engineering Co., Ine.}

\section{MEMORANDUM}

To F. E. Bingham THRU L. S. Sygitowicz

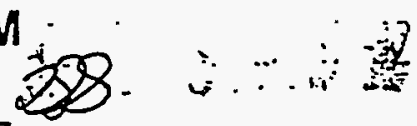

From G. A. Clark GAte

Date October 15, 1992

Subject

SAMPLE ANALYTICAL RESULTS

Enclosed are Analytical Services Department's results for the volatile organics, semivolatile organics, PCB organics, total petroleum hydrocarbons, total metals, pH, and flashpoint analyses of five water/oil samples collected on September 16, 1992, in Areas 11 and 12. These analyses were requested by

D. Madsen.

Please direct any questions you may have about these analyses to Sample Management (Roger Mitche11, 295-7220 or Jerry Dugas, 295-7997).

GAC:RNL663:rn

Enclosures

As stated

cy: Central Files, w/o encls.

H. A. Hawthorne, w/o encls.

D. Madsen, $w /$ encls. $x=$

ACS Packet No. 92-09-048, w/o encls. 


\section{REECO}

FRANK BIHGHAH

EHVIROHHENTAL COMPLIANCE

H/S 791

Attn: DAVE HADSEN

Purchase Order: 3754-009

Invoice Number: 911762
Order \#: 92-09-048

Date: 10/14/92 14:07

Hork ID: BHA, PCB, VOA,TPH, FLSHP, PH; A19

Date Received: $09 / 97 / 92$

Date Completed: $10 / 93 / 92$

Client Code: ENV_COHPLY

REPORT TO DAVE MADSEH, ENV_COMPLY.

SAMPLE IDENTIFICATION

\begin{tabular}{|c|c|c|c|}
\hline $\begin{array}{l}\text { Sample } \\
\text { Number }\end{array}$ & \multicolumn{3}{|c|}{ Sample } \\
\hline 01 & $11-1-1-T K$ & & \\
\hline 03 & $19-1-1-T K$ & & \\
\hline 04 & $11-1-1-T K$ & & JRGANICC \\
\hline 05 & $19-1-1-T K$ & & JRGANIC \\
\hline 06 & $11-1-1-T K$ & & \\
\hline 07 & $11-1-1-T K$ & & \\
\hline 08 & $11-2-2-D R U M$ & & \\
\hline 09 & $11-2-2-D R U M$ & & \\
\hline 10 & $11-2-2-D R U H$ & & \\
\hline 11 & $11-2-1-D R U H$ & & $01 \mathrm{~L}$ \\
\hline 12 & $11-2-1-D R U M$ & & JRGAH IC \\
\hline 13 & $11-2-1-D R U M$ & & 011 \\
\hline 14 & $25-0 G S / 6-Y A$ & RINSATE & WATER \\
\hline 15 & $25-0 G S / 6-Y A$ & RINSATE & HATER \\
\hline 23 & $12-13-1-T K$ & , & \\
\hline
\end{tabular}

\begin{tabular}{|c|c|c|c|}
\hline $\begin{array}{l}\text { Sample } \\
\text { Number }\end{array}$ & $\begin{array}{r}\text { Sampl } \\
\text { Descrip }\end{array}$ & $\begin{array}{l}e \\
\text { tion }\end{array}$ & \\
\hline 24 & $12-13-1-T K$ & & \\
\hline 25 & 92SAB0916-1 & TRIP & BLAHK \\
\hline 26 & $92-09-048-0 c 1$ & HETHOD & BLAHK \\
\hline 27 & $92-09-048-0 C 2$ & & \\
\hline 28 & $92-09-048-0 \mathrm{c} 3$ & HSD & \\
\hline 33 & $92-09-048-0 c 7$ & HS & \\
\hline 34 & $92-09-048-0<8$ & HSO & \\
\hline 35 & $92-09-048-0<9$ & HETHOD & BLAKK \\
\hline 36 & $92-09-048-a c 10$ & $O$ PCB C & OHTROL \\
\hline 37 & $92-09-048-0<1$ & DUP & LICATE \\
\hline 38 & $92-09-048-0 C 12$ & PREP & BLAKK \\
\hline 39 & $92-09-048-0<13$ & CAL & VER I F \\
\hline 40 & $92-09-048-0<14$ & 4 HS & \\
\hline
\end{tabular}

ANIC

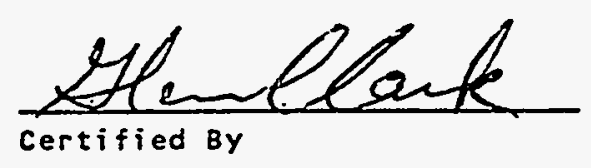


Sample Description: 25-OGS/6-YA RIHSATE MATER Lab Ho: 15A

Test Description: TOTAL METALS SCREEN, TCLP Method: ICP/COLD VAP Tes.t Code: TOTHTL Collected: $09 / 96 / 92$ Category: TOTAL_METALS

\begin{tabular}{|c|c|c|c|}
\hline PARAMETER & METHOD & RESULT & DET LIMIT \\
\hline ARSEHIC (AS) & 6010 & 0.12 & 0.028 \\
\hline BARIUM (Ba) & 6010 & 0.20 & 0.0009 \\
\hline CADHIUM (Cd) & 6010 & 0.060 & 0.0043 \\
\hline CHROMIUM (Cr) & 6010 & $<0.020$ & 0.0061 \\
\hline LEAD (Pb) & 6010 & 44 & 0.031 \\
\hline HERCURY (Hg) & $7470-1$ & 0.00072 & 0.00013 \\
\hline SELEHIUM (Se) & 6010 & MD & 0.066 \\
\hline SILVER (Ag) & 6010 & ND & 0.0048 \\
\hline
\end{tabular}

Hotes and Definitions for this Report:

EXTRACTED $09 / 22 / 92$

DATE RUN $09 / 29 / 92$

ANALYST AJK

UHITS MG/L 
Order \# 92-09-048

$10 / 14 / 92 \quad 14: 07$
REECO ASD/ACS

TEST RESULTS BY SAMPLE
Page 3

Sample Description: 92-09-048-0c19 HS

Lab Ho: $45 \mathrm{~A}$

Test Description: TOTAL METALS SCREEN, TCLP Category: TOT_MTLS_QCP Test COde: TOTHTL

\begin{tabular}{|c|c|c|c|}
\hline PARAMETER & METHOO & RESULT & DET LIMIT \\
\hline ARSENIC (AS) & 6010 & 89 & - \\
\hline BARIUM (Ba) & 6010 & 91 & 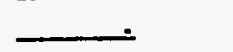 \\
\hline CADMIUM (Cd) & 6010 & 90 & 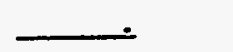 \\
\hline CHROMIUH (Cr) & 6010 & 91 & $=$ \\
\hline LEAD (Pb) & 6010 & 52 & - \\
\hline MERCURY (Hg) & $7470-1$ & 97 & \\
\hline SELENIUH (Se) & 6010 & . 98 & \\
\hline \multirow[t]{6}{*}{ SILVER (Ag) } & 6010 & 90 & 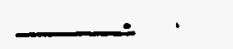 \\
\hline & \multicolumn{3}{|c|}{ Hotes and Definitions for this Report: } \\
\hline & EXTRACTED & \multicolumn{2}{|c|}{$09 / 22 / 92$} \\
\hline & DATE RUH & \multicolumn{2}{|c|}{$09 / 29 / 92$} \\
\hline & ANALYST & \multicolumn{2}{|c|}{ JKK } \\
\hline & UNITS & \multicolumn{2}{|l|}{ \% RECOVERY } \\
\hline
\end{tabular}


Sample Description: 92-09-048-0C20 METHDO BLHK Lab No: 46A

Test Description: TOTAL METALS SCREEN, TCLP Category: TOT_HTLS_QCP Test Code: TOTHTL

\begin{tabular}{|c|c|c|c|}
\hline PARAMETER & METHOO & RESULT & DET LIHIT \\
\hline ARSENIC (AS) & 6010 & ND & 0.028 \\
\hline BARIUH (Ba) & 6010 & 0.0065 & 30.0009 \\
\hline CADHIUH (Cd) & 6010 & ND & 0.0043 \\
\hline CHROMIUM (Cr) & 6010 & ND & 0.0061 \\
\hline LEAD (Pb) & 6010 & ND & 0.031 \\
\hline MERCURY (Hg) & $7470-1$ & ND & 0.00013 \\
\hline SELENIUM (Se) & 6010 & ND & 0.066 \\
\hline SILVER (Ag) & 6010 & HD & 0.0048 \\
\hline
\end{tabular}

Notes and Definitions for this Report:

EXTRACTED

DATE RUN $\quad 09 / 29 / 92$

ANALYST A.JK

UNITS

$M G / L$. 
Sample Description: 92-09-048-CV INST CHK SPK Lab Ho: 47A

Test Description: TOTAL METALS SCREEN, TCLP Category: TOT_MTLS_aCP Tesț Code: TOTMTL

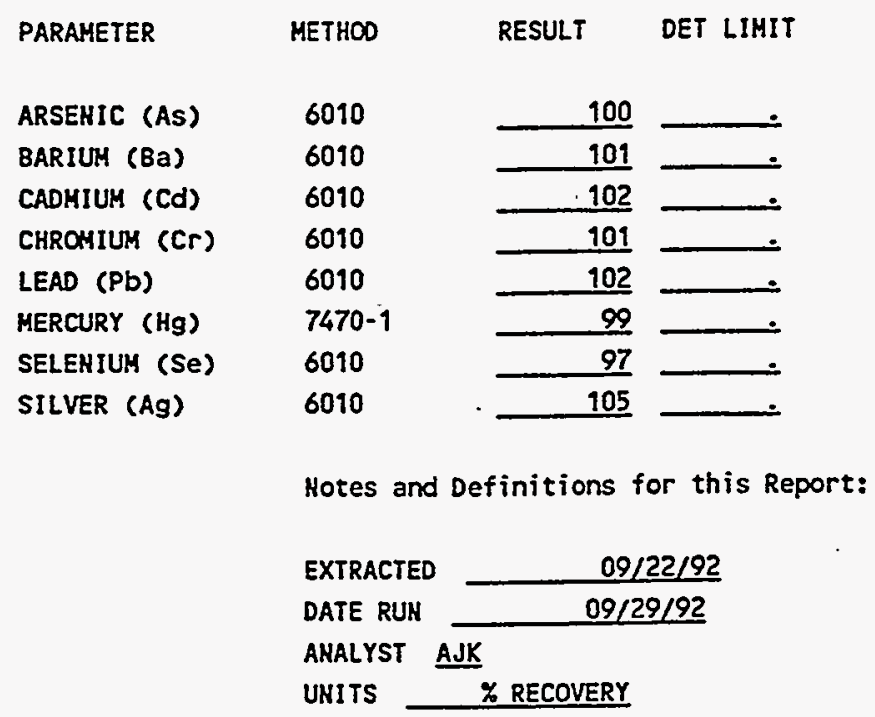


BASE/KEUTRAL/ACID ORGAHICS

Hethod: GC_MS

\begin{tabular}{|c|c|c|}
\hline $01 A$ & $11-1-1-T K$ & \\
\hline 018 & $11-1-1-T K$ & \\
\hline $18 \mathrm{~A}$ & $12-13-1-T K$ & \\
\hline $18 B$ & $12-13-1-T K$ & \\
\hline $26 A$ & $92-09-048-0 C 1$ & HETHOD \\
\hline $27 B$ & $92-09-048-0,2$ & MS \\
\hline 28B & $92-09-048 \cdot 0 \mathrm{cc} 3$ & MSO \\
\hline
\end{tabular}

FLASH POIHT

Hethod: PEHSKYHART IN

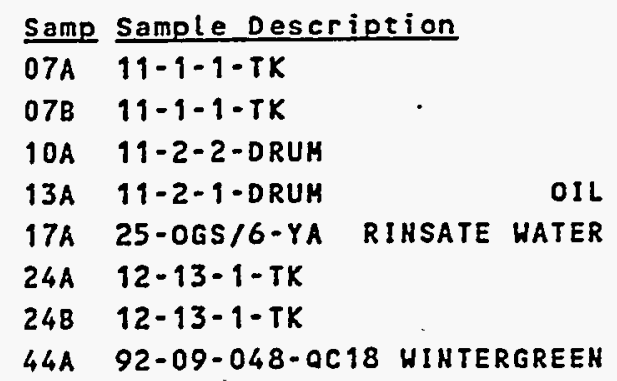

PCB 1242

Hethiod: GC_ECD

$\begin{array}{llr}\frac{\text { Samp }}{\text { 04A }} & \text { Sample Description } & \\ 11-1-1-T K & \text { ORGAHIC } \\ \text { 04B } & 11-1-1-T K & \text { AQUEOUS } \\ 21 A & 12-13-1-T K & \text { ORGAKIC } \\ 21 B & 12-13-1-T K & \text { AQUEOUS } \\ 36 A & 92-09-048-\text { QC10 PCB CONTROL } \\ 37 A & 92-09-048-Q C 11 & \text { DUPLICATE } \\ 38 A & 92-09-048-Q C 12 & \text { PREP BLANK }\end{array}$

PCB 1254

Hethod: GC_ECD

\begin{tabular}{|c|c|c|c|}
\hline $04 A$ & $11-1-1-T K$ & & ORGAH IC \\
\hline 04 & $11-1-1-T K$ & & AQUEOUS \\
\hline $21 \mathrm{~A}$ & $12-13-1-T K$ & & ORGAH IC \\
\hline 218 & $12-13-1-T K$ & & AQUEOUS \\
\hline $36 A$ & $92-09-048-0<10$ & PCB & CONTROL \\
\hline $7 A$ & $92-09-048-0,11$ & & UPLICATE \\
\hline $38 A$ & $92-09-048-0 C 12$ & PRE & EP BLANK \\
\hline
\end{tabular}

$\begin{array}{ll}\text { Minimum: } & \text { Maximum: } \\ & \\ \text { ReSult } & \text { Units Limit Prepared Analyzed By } \\ \text { SEE DATA } & \text { PACKAGE } \\ \text { SEE DATA } & \text { PACKAGE } \\ \text { SEE DATA } & \text { PACKAGE } \\ \text { SEE DATA } & \text { PACKAGE } \\ \text { SEE DATA } & \text { PACKAGE } \\ \text { SEE DATA } & \text { PACKAGE } \\ \text { SEE DATA } & \text { PACKAGE }\end{array}$

Minimua: Maximun:

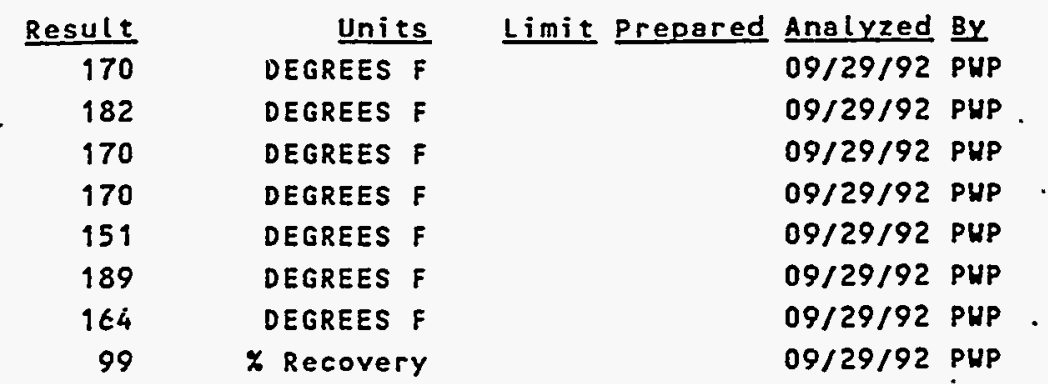

Hinimum: $\quad 0.0$ Maximum: 999999.0

\begin{tabular}{rrr} 
Result & Units & Limit Prepared Analyzed BY \\
\hline$<2.5$ & ppm & \\
TEXT & Ppm \\
2.5 & Ppm \\
$T E X T$ & Ppm \\
111 & \% RECOVERY \\
$<2.5$ & Ppm \\
$<2.5$ & Ppm
\end{tabular}

Kinimun: $\quad 0.0$ Maximua: 999999.0

\begin{tabular}{rrr} 
Result & Units & Limit Prepared Analyzed By \\
\hline 22.5 & Ppm & \\
TEXT & PPm \\
2.5 & Ppm \\
TEXT & Ppm \\
102 & K RECOVERY \\
$<2.5$ & PPm \\
$<2.5$ & Ppm
\end{tabular}


order $92-09-048$

10/14/92 14:07
REECO ASD/ACS

REGULAR TEST RESULTS.BY TEST
Page 7
PCB 1260

Hethod: GC_ECD

Samp Sample Description

04A $11-1-1-T K$

$04 B \quad 11-1-1-T K$

ORGANIC AQUEOUS

ORGAKIC

AQUEOUS

$21 B \quad 12-13-1-T K$

36A 92-09-048-0C10 PCB CONTROL

37A 92-09-048-0C11 DUPLICATE

38A 92-09-048-QC12 PREP BLAHK

TPH - DIESEL

Method: GC

Samp Sample Description

05A $11-1-1 \cdot$ TK

O5B $11-1-1-T K$

ORGANIC

AQUEOUS

O9A 11-2-2-DRUH

22A $12-13-1-T K$

22B $12-13-1-T K$

ORGANIC

AQUEOUS

39A 92-09-048-0C13 CAL VERIF

40A 92-09-048-0C14 HS

41A 92-09-048-QC15 DUPLICATE

42A 92-09-048-QC16 METHOD BLNK

TPH - GASOLIHE

Hethod: GC

Samp Sample Description

05A 11-1-1-TK

05B $11-1-1-T K$

ORGAMIC

AQUEOUS

O9A 11-2-2-DRUH

12A $11-2-1-D R U M$

ORGANIC

16A 25-OGS/6-YA

RINSATE WATER

22A 12-13-1-TK

ORGANIC

22B 12-13-1-TK

AQUEOUS

39A 92-09-048-0C13 CAL VERIF

40A 92-09-048-QC14 HS

$41 A$ 92-09-048-0C15 DUPLICATE

$42 A$ 92-09-048-QC16 HETHOD BLNK

TPH - OIL

Method: GC

Samp Sample Description

05A 11-1-1-TK

05B 11-1-1-TK

O9A $11-2-2-D R U M$

22A $12-13-1-T K$
Minimum:

Result

$<2.5$

TEXT

$<2.5$

TEXT

88

$<2.5$

$<2.5$

Minimum:

Result

854,000

11,000

807,000

913,000

14,200

98

12,400

794,000

$<10.0$

Mininum:

Result

59.4

$<5.0$

98.8

137

$<5.0$

167

24.0

105

120

55.9

$<5.0$

Minimua:

Result

$<10.0$

$<0.5$

$<10.0$

$<10.0$
0.0 Maxinua: $\$ 999999.0$

Units Limit Prepared Analyzed By

ppm

ppm

ppm

ppm

* RECOVERY

ppm.

ppm

o Maxinum: 999999999

Units Limit Prepared Anslyzed By $\mathrm{mg} / \mathrm{kg}$

$\mathrm{mg} / \mathrm{l}$

$\mathrm{mg} / \mathrm{kg}$

$\mathrm{mg} / \mathrm{kg}$

$\mathrm{mg} / \mathrm{l}$

* recovery

$\mathrm{mg} / \mathrm{l}$

$\mathrm{mg} / \mathrm{kg}$

$\mathrm{mg} / \mathrm{kg}$

o Maximum: 999999999

Units Limit Prepared Analyzed By

mg/kg

$\mathrm{mg} / \mathrm{kg}$

$\mathrm{mg} / \mathrm{kg}$

$\mathrm{mg} / \mathrm{kg}$

$\mathrm{mg} / \mathrm{kg}$

$\mathrm{mg} / \mathrm{kg}$

$\mathrm{mg} / \mathrm{kg}$

* RECOVERY

X Recovery

$\mathrm{mg} / \mathrm{kg}$

$\mathrm{mg} / \mathrm{kg}$

o Haxinun: 999999999

Units Limit Prepared Analyzed By $\mathrm{mg} / \mathrm{kg}$

$\mathrm{mg} / \mathrm{l}$

$\mathrm{mg} / \mathrm{kg}$

$\mathrm{mg} / \mathrm{kg}$ 
Order $\$ 92-09-048$

$10 / 14 / 92 \quad 14: 07$
REECO ASD/ACS

REGULAR TEST RESULTS. BY TEST
Page 8
Samp Sample Description

22B 12-13-1-TK AQUEOUS

39A 92-09-048-0C13 CAL VERIF

40A 92-09-048-0C14 HS

41A 92-09-048-QC15 DUPLICATE

42A 92-09-048-QC16 METHOD BLNK

TPH - TOTAL.

Hethod: GC

Samp Sample Description

05A 11-1-1-TK

OSB 11-1-1-TK

O9A $11-2-2-D R U A$

22A 12-13-1-TK

$22 B \quad 12-13-1-T K$

41A 92-09-048-0C15 DUPLICATE

42A 92-09-048-0C16 METHOD BLHK

VOLATILE ORGAKIC AHALYSIS

Method: GC_MS

\section{Samp Sample Description}

03A 11-1-1-TK

O3B, 11-1-1-TK

O8A 11-2-2-DRUH

$11 A$ 11-2-1-DRUM

$14 A$ 25-OGSI6-YA RINSATE HATER

20A $12-13-1-T K$

20B $12-13-1-T K$

25A 925AB0916-1

27A 92-09-048-0C2

28A 92-09-048-0C3 HSD

$33 A$ 92-09-048-0C7 HS

34A 92-09-048-QC8 MSD

35A 92-09-048-0C9 METHOD BLANK

pH

Method: METER

Samp Sample Description

06A 11-1-1-TK

O6B 11-1-1-TK

23A 12-13-1-TK

$23 B \quad 12-13-1-T K$

43A $92-09 \cdot 048-0 C 17$

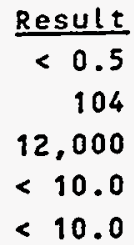

Minimua:

Result
854,000
11,000
807,000
913,000
14,200
794,000
$<25.0$

Minimun:

Result

SEE DATA

SEE DATA

SEE DATA

SEE DATA

SEE DATA

SEE DATA

SEE

SEE DATA

SEE DATA

SEE DATA

SEE DATA

SEE DATA

SEE DATA

Minimum:

Result NONE HOME HOHE HONE BUFFERS

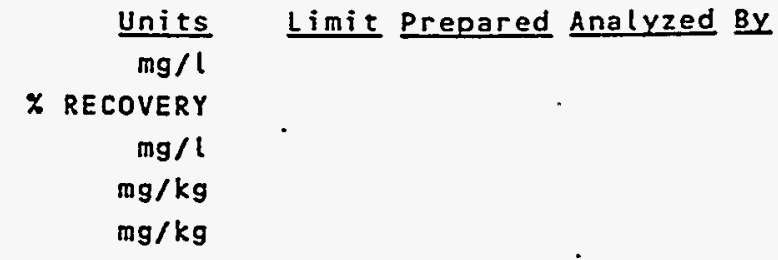

O Maximum: 999999999

Units Limit Prepared Anslyzed By $\mathrm{mg} / \mathrm{kg}$

$\mathrm{mg} / \mathrm{l}$

$\mathrm{ing} / \mathrm{kg}$

$\mathrm{mg} / \mathrm{kg}$

$\mathrm{mg} / \mathrm{l}$

$\mathrm{mg} / \mathrm{kg}$

$\mathrm{mg} / \mathrm{kg}$

Maximug:

Units Limit Prepared Analyzed By

PACKAGE

PACKAGE

PACKAGE

PACKAGE

PACKAGE

PACKAGE

DATA PACKAGE

PACKAGE

PACKAGE

PACKAGE

PACKAGE

PACKAGE

PACKAGE

o Maximun:

14

Units Limit Prepared Analyzed By SEE COMHEHTS

09/17/92 LDR $09 / 17 / 92$ LDR $09 / 17 / 92$ LDR $09 / 17 / 92$ LDR $09 / 17 / 92$ LDR 
WET CHEMISTRY ANALYST'S COMMENTS

SAMPLE 11-1-1-TK AND 12-13-1-TK HERE ORGANIC AND THE PH COULO NOT BE MEASURED.

TPH AHALYST'S NOTES:

ALL SAMPLES ANALYSED EXHIBITED A GENERAL CHROMATOGRAM IHDICATIHG THE PRESENCE OF DIESEL. MOST. ANALYSES HERE DOHE ON ORGAHIC LIOUIDS, HHICH HERE HEIGHED INTO VOLUMETRIC FLASKS AND DILUTED TO VOLUME HITH THE SOLVENT. FOR THIS REASOH, THE ORGANIC LIQUID RESULTS ARE REPORTED HITH THE UMITS "HG/KG".

SAMPLES 11-2-1-DRUH AND 25-OGS/6-YA WERE ANALYSED ONLY FOR GASOLIHE, BUT ALSO EXHIBIT PEAKS OF HEAVIER HYDROCARBONS.

HATRIX SPIKE SAMPLES HERE PREPARED FOR THE AQUEOUS SAMPLES AMD DUPLICATE ANALYSES WERE DONE FOR THE ORGANIC LIOUIDS. DUE TO THE LEVEL OF DIESEL IN THE AQUEOUES SAMPLES, THE MATRIX SPIKE RESULTS SHOULD BE CONSIDERED DUPLICATE ANALYSES. THE GASOLINE MATRIX SPIKE HAS AFFECTED BY THE AMOUNT OF DIESEL IN THE LIQUID AHD RETURNED SLIGHTLY HIGHER THAN USUAL. FOR THIS REASOH, CALIBRATION VERIFICATION STAHDARDS HERE RUH AHO THE RESULTS REPORTED IH ORDER TO VALIDATE THE AHALYSES. ALL DUPLICATE ORGAHIC SAHPLES MERE PREPARED FROM SAHPLE $11-1-1-T K$.

PCB ANALYST'S MOTES:

SAMPLES 19-1-1-TK AND 12-13-1-TK CONTAINED BOTH AN AQUEOUS AND ORGAKIC PHASE. AN ALIQUOT OF THE ORGANIC PORTION OF BOTH SAMPLES WAS PREPARED FOR PCB ANALYSIS AS WELL AS THE AQUEOUS FRACTION OF 11-1-1-TK. INSUFFICIENT SAMPLE WAS SUBMITTED FOR EXTRACTION OF THE AQUEOUS PHASE OF SAMPLE 12-13-1-TK.

AHALYSIS OF THE AQUEOUS EXTRACT PRODUCED A LARGE RESPONSE, BUT HO IDENTIFIABLE AROCLOR PATTERNS ARE OBSERVED. THE CHROHATOGRAHS OF THE ORGAHIC EXTRACTS ALSO EXHIBIT SIGHIFICANT RESPONSES, BUT NO AROCLOR PEAKS ARE PRESENT.

HO VALUES ARE REPORTED FOR THE AQUEOUS EXTRACT OF SAMPLE 11-1-1-TK AS HO METHOD DETECTION LIMITS HAVE BEEN DETERMINED ACCORDIHG TO METHOD 8080. 
VOLATILES DATA 
$\rightarrow$ 
ANALYST NOTES: VOAS

- Date: September 30, 1992

Lab Work Order No.:92-09-048

lient sample Id.: 11-1-1-TR (03A, 03B), 11-2-2 DRUM, 11-2-1

DRUM, 25-OGS/6-YA, 12-13-1-TR (20A,20B), 92SAB0916-1 (TB). Analyst: Philip Briggs

The samples were analyzed on a Hewlett Packard 5890 GC/5970 MSD, with a Tekmar purge and trap connected for direct injection on column. Instrumental parameters used are those specified in SW-846 method 8240. The target analytes are listed with their CAS numbers, and the amount of the target compound found in ug/L or ug/Kg, depending on the matrix. If the analyte was not found in the sample, the quantitation limit is reported followed by an $n . d$. qualifier. The qualifiers used in this reporting format are:

n.d.-Indicates compound was analyzed for but not detected.

$J$ - Indicates an estimated value. Indicates the presence of a compound that meets the identification criteria, but the result is less than the sample quantitation limit but greater than zero.

$B$ - This flag is used when the analyte is found in the associated blank as well as in the sample. It indicates possible/probable blank contamination.

E- This flag identifies compounds whose concentrations exceed the calibration range of the GC/MS instrument for that specific analysis.

D- This flag identifies all compounds identified in an analysis at a secondary dilution factor.

The volatile compounds methylene chloride, acetone, and 2butanone are considered to be common laboratory contaminants by the U.S. EPA. The U.S. EPA Contract Laboratory Program (CLP) Statement of Work for Organic Analysis, OLMO1.1-.6, dated June 1991, Exhibit D, Section IV, paragraph 10.10 allows concentrations up to five times (5X) the contract Required Quantitation Limit (CRQL) of $10 \mathrm{ppb}$ (ie $50 \mathrm{ppb}$ is allowed) of the common laboratory contaminants methylene chloride, acetone and 2-butanone to be present in the method blank before reanalysis is required. If these contaminants are found in samples as well as the method blank, they are to be qualified by a " $B$ " to show they are possible laboratory contamination.

The matrix spike runs used 11-1-1-TK for the oils and 25OGS/6-YA for the water analyses. 
REECO ANALYTICAL CHEM DOTK LAB'S VOLATILE ORGANICS TेंLUT RESULTS

Name Field: $11-1-1-T K$ W

Blank:>VD784:PASS QaQC File: QVOLWM Misc Field: 92-09-048-03A 5.0 ML WATER SAMPLE

Instrument: VOAI Datafile: >VD787

Idfile: ID_VCl

Quantfile:

^VD787

Injection time: $16: 43$ on 09-23-92

Quant Time: $17: 16$ on 09-23-92

Continuing calibration time : 08:19 on 09-23-92 using the file: AVW318::DI

User dilfac: 1 Quant dilfac: 1 Multi-calibration: 14:49 on 05-12-92

Compound Name

$==========$
Chloromethane

Bromomethane

Vinyl Chloride

Chloroethane

Methylene Chloride

Acetone

Carbon Disulfide

1,1-Dichloroethene

1,1-Dichloroethane

1,2-Dichloroethene (total)

trans-1,2-Dichloroethene

Chloroform

1,2-Dichloroethane

2-Butanone

$1,1,1$-Trichloroethane

Carbon Tetrachloride

Vinyl Acetate

Bromodichloromethane

1,2-Dichloropropane

cis-1, 3-Dichloropropene

Trichloroethene

Dibromochloromethane

1,1,2-Trichloroethane

Benzene

trans-1,3-Dichloropropene

Bromoform

4-Methy 1-2-Pentanone

2-Hexanone

Tetrachloroethene

$1,1,2,2$-Tetrachloroethane

Toluene

Chlorobenzene

Ethylbenzene

styrene

In, $\mathrm{p}$-XYlene

xylene (total)

n.d. - not detected

Approved by:

\section{CAS Number}

Result

$74-87-3$

$74-83-9$

$75-01-4$

$75-00-3$

$75-09-2$

67-64-1

75-15-0

75-35-4

75-34-3

540-59-0

540-59-0

67-66-3

$107-02-2$

78-93-3

$71-55-6$

56-23-5

108-05-4

$75-27-4$

78-87-5

10061-01-5

79-01-6

$124-48-1$

79-00-5

$71-43-2$

10061-02-6

75-25-2

108-10-1

591-78-6

127-18-4

$79-34-5$

108-88-3

108-90-7

$100-41-4$

100-42-5

133-02-7

$133-02-7$ n.d.

n.d.

n.d.

n.d.

n.d.

$200 . B$

n.d.

n.d.

n.a.

n.d.

n.d.

n.d.

6.

37.

35 .

n.d.

n.d.

n.d.

n.d.

n.d.

n.d.

n.d.

n.d.

67 .

n.d.

n.d.

n.d.

n.d.

n.d.

n.d.

200 .

n.d.

58.

n.d.

240 .

150 .

Detection

Units Limit

UG/I $\quad 10$.

UG/L 10 .

UG/I 10 .

UG/L 20.

UG/L

UG/L

UG/I

UG/L

UG/L

UG/I

UG/I

$U G / I$

UG/I

$U G / I$

UG/I

UG/I

$U G / I$

$U G / L$

UG/I

UG/I

$U G / L$

UG/I

UG/L.

UG/L

UG/I

$U G / I$

$U G / L$

$U G / L$

UG/L

UG/I

UG/I

UG/L

UG/I

$U G / I$

$U G / I$

UG/I

5.

10.

5.

5.

5.

5.

5.

5.

5.

10.

5.

5.

10.

5.

5.

5.

5.

5.

5.

5.

5.

5.

10.

10.

5.

5.

5.

5.

5.

5.

5.

5. 
Name Field: $11-1-1-\mathrm{TK}$ p

Blank:>VD784:PASS QaQC File: QVOLSM.

Misc Field: 92-09-048-03B 0.2GR/10CCMEOH//50UL ANALYZED

Instrument: VOAl Datafile: >VD786 Idfile: ID_VCI

Quantfile: ^VD786

Injection time: 16:03 on 09-23-92

Quant Time: $16: 35$ on $09-23-92$

continuing calibration time : 08:19 on 09-23-92 using the file: AVW318::DI User dilfac:5000 Quant dilfac: 5000. Multi-calibration: 14:49 on 05-12-92

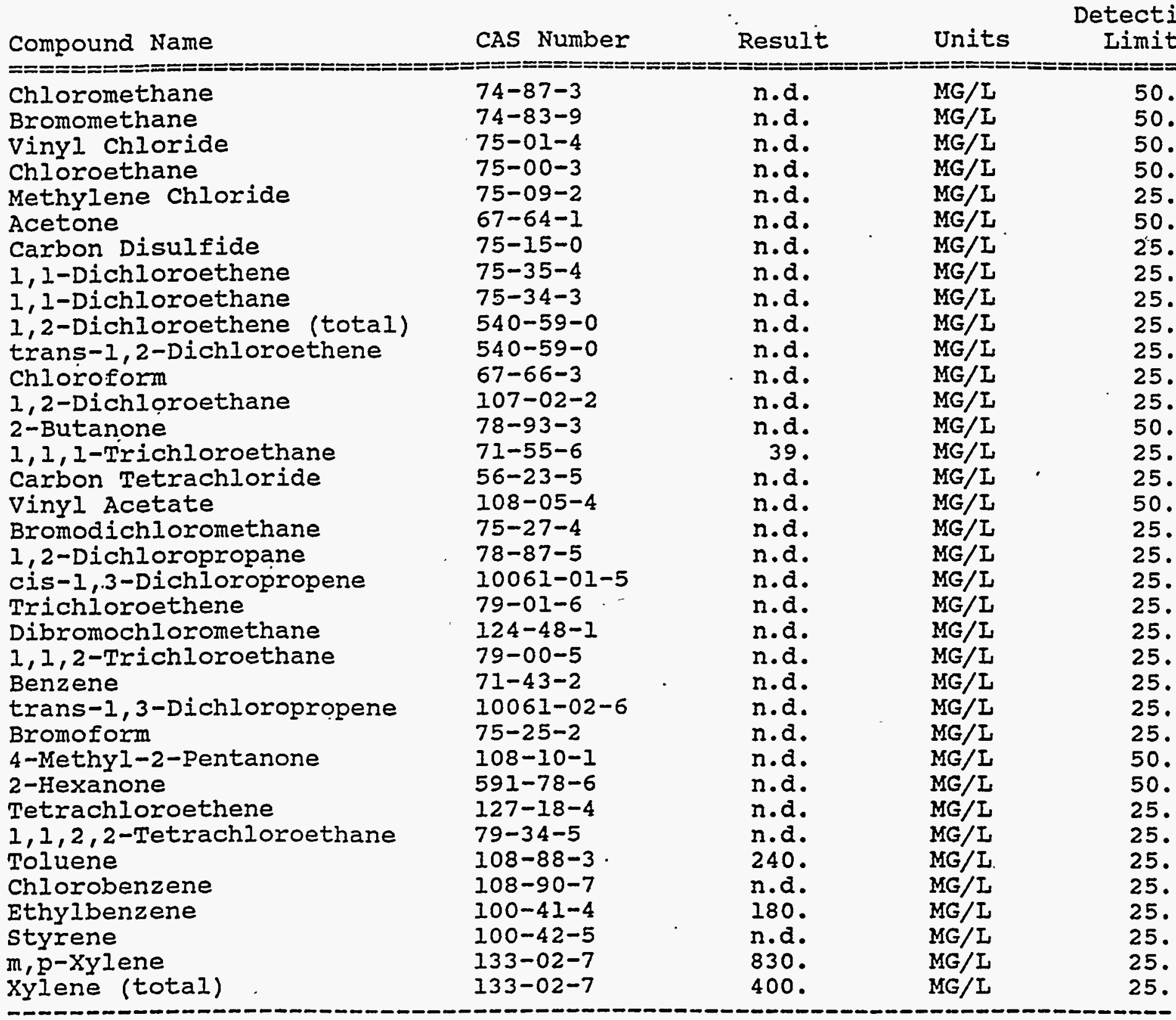

n.d. - not detected

Approved by: 
REECO ANALYTICAL CHEN._STRY LAB'S VOLATILE ORGANLL TË¿̈: RESULTS

Name Field: $11-1-1-T K$ P MS Misc Field: Instrument:
$92-09-048-33 A$

VOA1

Datafile: >AD799

Blank: >AD790: PASS

GR/IOCC//5OUL

Idfile: ID VCl
QaQC File: QVOLSM

Quantfile: ^AD799

Injection time: 19:12 on 09-24-92

Continuing calibration time : $10: 16$ on $09-24-92$

Quant Time: 11:08 on 09-25-92 User dilfac:5000 Quant dilfac: 5000. Multi-calibration: 14:49 on 05-12-92

Compound Name

Chloromethane

Bromomethane

vinyl Chloride

Chloroethane

Methylene Chloride

Acetone

Carbon Disulfide

1,I-Dichloroethene

I, 1-Dichloroethane

$1,2-$ Dichloroethene (total)

trans-1,2-Dichloroethene

Chloroform

1,2-Dichloroethane

2-Butanone

$1,1,1$-Trichloroethane

Carbon Tetrachloride

Vinyl Acetate

Bromodichloromethane

1,2-Dichloropropane

cis-1,3-Dichloropropene

Trichloroethene

Dibromochloromethane

1,1,2-Trichloroethane

Benzene

trans-1,3-Dichloropropene

Bromoform

4-Methyl-2-Pentanone

2-Hexanone

Tetrachloroethene

$1,1,2,2$-Tetrachloroethane

Toluene

Chlorobenzene

Ethylbenzene

styrene

$\mathrm{m}, \mathrm{p}$-Xylene

xylene (total)
CAS Number

$74-87-3$

$74-83-9$

$75-01-4$

$75-00-3$

$75-09-2$

$67-64-1$

75-15-0

$75-35-4$

$75-34-3$

540-59-0

540-59-0

67-66-3

107-02-2

$78-93-3$

$71-55-6$

$56-23-5$

$108-05-4$

$75-27-4$

$78-87-5$

$10061-01-5$

79-01-6

$124-48-1$

$79-00-5$

71-43-2

10061-02-6

$75-25-2$

108-10-1

$591-78-6$

127-18-4

$79-34-5$

108-88-3

108-90-7

$100-41-4$

$100-42-5$

$133-02-7$

133-02-7
Result

n.d.

n.d.

n.d.

n.d.

n.d.

n.d.

n.d.

210 .

n.d.

46.

n.d.

n.d.

$n \cdot d$.

n.d.

37.

n. d.

n.a.

n.d.

n.d.

n.d.

260 .

n.d.

n.d.

240 .

n. $a$.

n.d.

n.d.

n.d.

31 .

n.d.

450 .

250 .

160 .

n.a.

660 .

340 .

Detectio

Iimit

Units

MG/I

$M G / I$

$\mathrm{MG} / \mathrm{I}$

$M G / L$

MG/L

$M G / I$

$M G / L$

MG/L

MG/I

MG/L

$\mathrm{MG} / \mathrm{I}$

$\mathrm{MG} / \mathrm{I}$

$M G / L$

$M G / I$

MG/L

$M G / L$

$M G / I$

$M G / I$

$\mathrm{MG} / \mathrm{I}$

MG/I

$\mathrm{MG} / \mathrm{I}$

$M G / L$

MG/I

$M G / I$

$M G / I$

$\mathrm{MG} / \mathrm{I}$

MG/I

MG/L

$M G / I$

$M G / L$

$M G / I$

MG/I

$M G / I$

$M G / I$

MG/L

$M G / I$
50.

50.

50 .

50 .

25.

50 .

25.

25 .

25 .

25 .

25 .

25.

25 .

50 .

25 .

25 .

50 .

25.

25 .

25.

25.

25.

25.

25.

25.

25.

50.

50.

25.

25.

25 .

25.

25.

25 .

25 .

25.

n.d. - not detected

Approved by: 


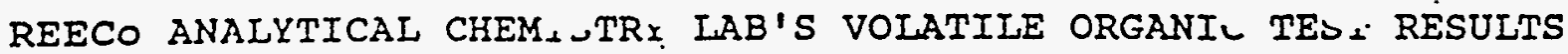

Name Fleld: 11-1-1-TK P MSD Blank: >VD790: PASS

Misc Field: 92-09-048-34A MTRX SPK DUPL .2 GR/I0CC//5OUL

Instrument: VOAl Datafile: >AD800 Idfile: ID_VCl Quantfile: AAD800

Injection time: $19: 50$ on 09-24-92

Quant Time: $11: 11$ on $09-25-92$

Continuing calibration time : $10: 16$ on 09-24-92 using the file: AVW320::DI

User dilfac:5000 Quant dilfac: 5000. Multi-calibration: 14:49 on 05-12-92

Compound Name

$==========$

Bromomethane

vinyl Chloride

Chloroethane

Methylene Chloride

Acetone

Carbon Disulfide

1,1-Dichloroethene

I, 1-Dichloroethane

1,2-Dichloroethene (total)

trans-1,2-Dichloroethene

Chloroform

1,2-Dichloroethane

2-Butanone

1, 1, 1-Trichloroethane

Carbon Tetrachloride

Vinyl Acetate

Bromodichloromethane

1,2-Dichloropropane

cis-1,3-Dichloropropene

Trichloroethene

Dibromochloromethane

1,1,2-Trichloroethane

Benzene

trans-1, 3-Dichloropropene

Bromoform

4-Methyl-2-Pentanone

2-Hexanone

Tetrachloroethene

$1,1,2,2$-Tetrachloroethane

Toluene

Chlorobenzene

Ethylbenzene

styrene

m, p-xylene

xylene (total)
CAS Number

$74-87-3$

$74-83-9$

$75-01-4$

$75-00-3$

$75-09-2$

$67-64-1$

$75-15-0$

$75-35-4$

$75-34-3$

540-59-0

540-59-0

$67-66-3$

107-02-2

$78-93-3$

$71-55-6$

$56-23-5$

108-05-4

$75-27-4$

78-87-5

$10061-01-5$

79-01-6

124-48-1

79-00-5

71-43-2

10061-02-6

75-25-2

108-10-1

591-78-6

$127-18-4$

$79-34-5$

$108-88-3$

108-90-7

$100-4 I-4$

$100-42-5$

133-02-7

133-02-7
Resuit

n. $\alpha$.

n.d.

n.d.

n.d.

n.d.

n.d.

n.d.

210 .

n.d.

43.

n.d.

n.d.

n.d.

n.d.

37.

n.d.

$n: d$.

n.d.

n.d.

n.d.

250 .

n.d.

n.d.

240 .

n.d.

n.d.

n.d.

n.d.

31.

n.d.

450 .

240 .

150 .

n.d.

660 .

320 .

Detectior.

Iimit

Units

$======$

$M G / L \quad 50$.

$M G / L$

50 .

$M G / L$

50 .

$M G / L$

50.

$M G / L$

25.

$M G / L$

50.

$M G / L$

25.

MG/L

25.

$M G / L$

25 .

$M G / L$

$M G / I$

25 .

25 .

25.

$M G / L$

$M G / I$

MG/I

$M G / I$

$M G / I$

$M G / L$

MG/I

MG/L

$\mathrm{MG} / \mathrm{L}$

$M G / L$

$M G / L$

MG/I

MG/L

$M G / I$

MG/I

$M G / I$

$M G / L$

$M G / I$

MG/I

$M G / I$

MG/I

MG/L

$M G / I$

25.

50 .

25.

25.

50 .

25.

25.

25.

25 .

25.

25 .

25.

25 .

25.

50 .

50.

25.

25 .

25.

25.

25.

25.

25.

25.

n.d. - not detected

Approved by: 
REECO ANALYTICAL CHEMISTRY LAB'S VOLATILE ORGANIC TES $\perp$ RESUITS

Name Field: $11-2-1$ DRUM

Blank: >AD790:PASS QAQC File: QVOLFM

Misc Fleld: 92-09-048-IIA $0.2 \mathrm{GR} / 10 \mathrm{CC} / / 50$ UL ANALYZED

Instrument: VOAI Datafile: >AD794 Idfile: ID VCI

Quantfile: ^AD794

Injection time: 16:03 on 09-24-92

Continuing calibration time : 10:16 on 09-24-92 using the file: AVW320::D1 User dilfac:5000 Quant dilfac: 5000. Multi-calibration: $14: 49$ on 05-12-92

Compound Name

$=========$
Chloromethane

Bromomethane

Vinyl chloride

Chloroethane

Methylene Chloride

Acetone

Carbon Disulfide

1,1-Dichloroethene

1,1-Dichloroethane

1,2-Dichloroethene (total)

trans-1,2-Dichloroethene

Chloroform

1,2-Dichloroethane

2-Butanone

$1,1,1$-Trichloroethane

Carbon Tetrachloride

Vinyl Acetate

Bromodichloromethane

1,2-Dichloropropane

cis-1,3-Dichloropropene

Trichloroethene

Dibromochloromethane

1, 1,2-Trichloroethane

Benzene

trans-1, 3-Dichloropropene

Bromoform

4-Methyl-2-Pentanone

2-Hexanone

Tetrachloroethene

$1,1,2,2$-Tetrachloroethane

Toluene

Chlorobenzene

Ethylbenzene

styrene

$\mathrm{m}, \mathrm{p}$-Xylene

xylene (total)
CAS Number

$74-87-3$

$74-83-9$

$75-01-4$

75-00-3

$75-09-2$

$67-64-1$

$75-15-0$

$75-35-4$

$75-34-3$

540-59-0

540-59-0

$67-66-3$

$107-02-2$

$78-93-3$

$71-55-6$

56-23-5

108-05-4

$75-27-4$

$78-87-5$

10061-01-5

$79-01-6$

$124-48-1$

79-00-5

$71-43-2$

10061-02-6

75-25-2

108-10-1

591-78-6

127-18-4

$79-34-5$

108-88-3

108-90-7

$100-41-4$

$100-42-5$

133-02-7

$133-02-7$
Result

n.d.

n.d.

n.d.

n.d.

n.d.

n.d.

n.d.

n.d.

n.d.

n.d.

n.d.

n.d.

n.d.

n.d.

n.d.

n.d.

n.d.

n.d.

n.d.

n.d.

n.d.

n.d.

n.a.

110 .

n.d.

n.d.

n.d.

n.d.

n.d.

n.d.

930.

n.d.

520.

n.d.

1400 .

860 .

Detection

Units Iimit

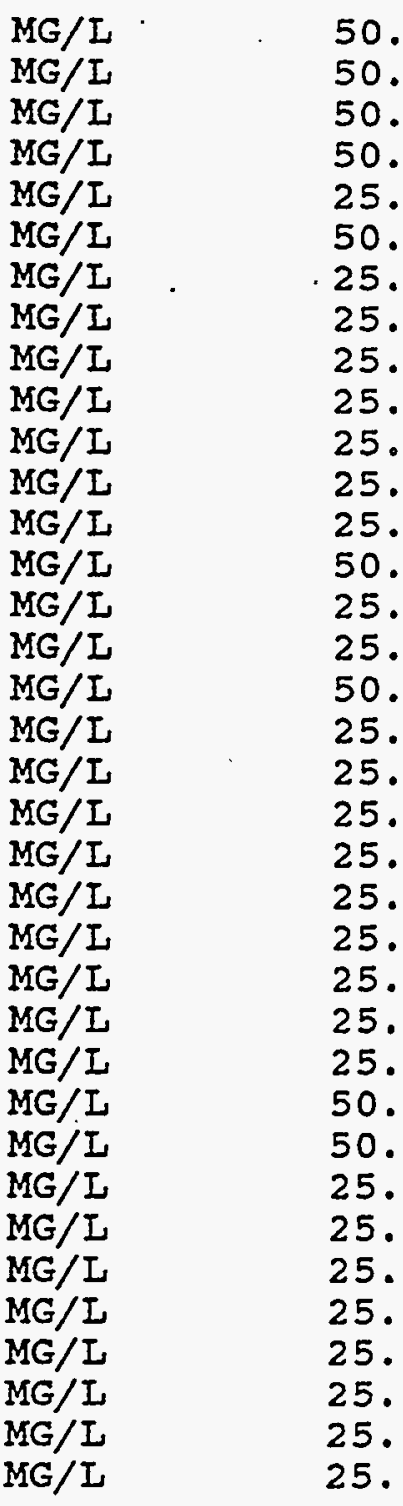

n.d. - not detected

Approved by: 
Name Field: $11-2-2$ DRUM

BIank: >AD790: PASS

Misc Fleld: 92-09-048-08A $0.2 \mathrm{GR} / 10 \mathrm{CC} / / 50$ UI ANALYZED

Instrument: VOAI Datafile: >AD793 Idfile: ID_VCI

QaQc. File: QVOLWM.

Instrument: VOAl

Injection time: $15: 25$ on 09-24-92

continuing calibration time : $10: 16$ on $09-24-92$

Quant Time: $15: 57$ on 09-24-92

using the file: ^VW320::DI

User dilfac:5000 Quant dilfac: 5000. Multi-calibration: 14:49 on 05-12-92

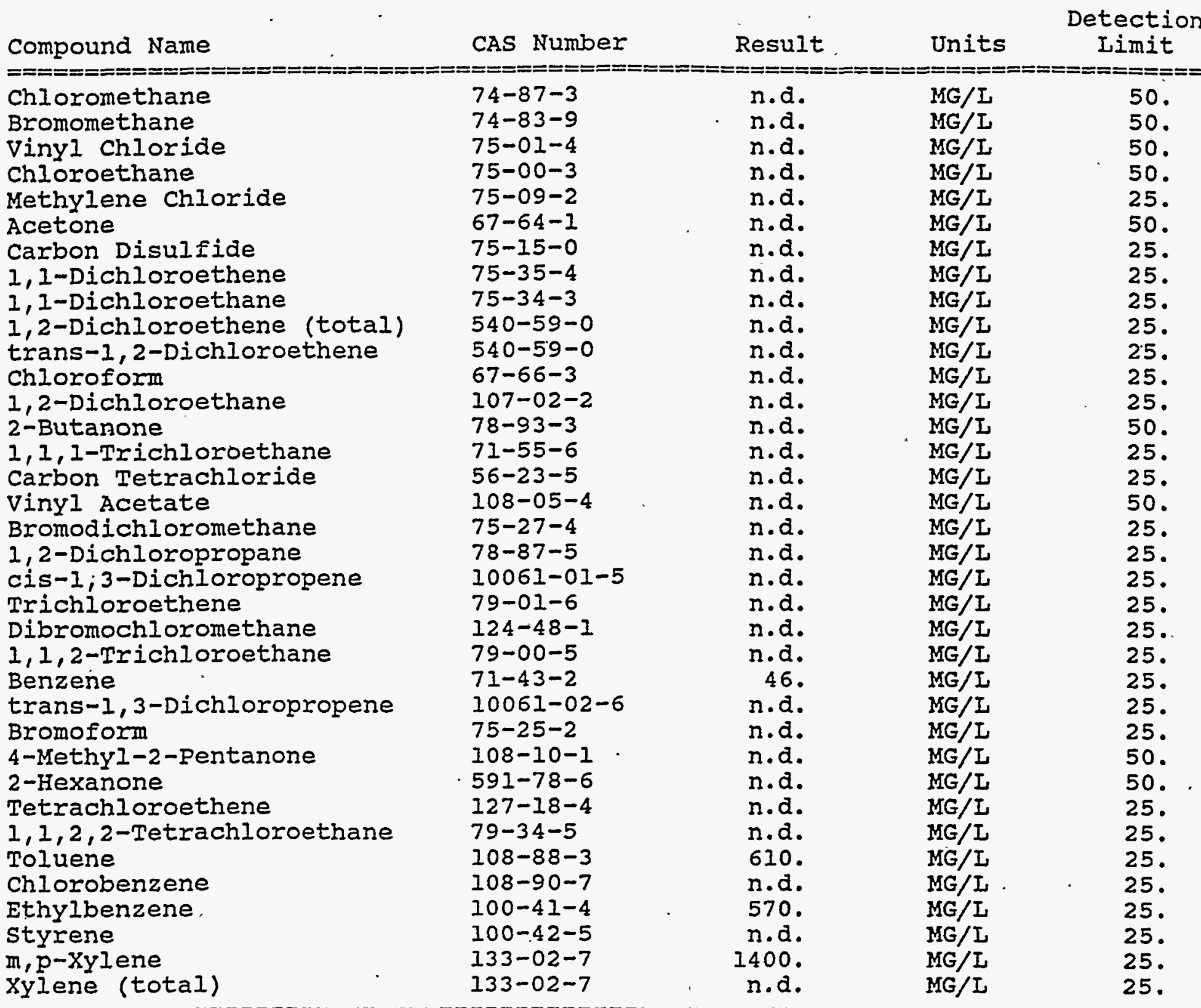

n.a. - not detected

Approved by: 
REECO ANALYTICAL CHEMISTR, LAB'S VOLATILE ORGANICS TLOT RESULTS

Name Field: 25-0GS/6-YA

Misc Field: 92-09-048-14A 5 MLS

Instrument: VOAl Datafile: $>$ VD788
Blank:>VD784:PASS QaQC File: QVOLWM Idfile: ID_VCI Quantfile: ^VDi88
Injection time: $17: 25$ on $09-23-92$

Continuing calibration time : 08:19

User dilfac: I

Quant dilfac:
Quant Time: $17: 57$ on 09-23-92 on 09-23-92 using the file: $\wedge$ VW318::DI
1. Nulti-calibration: $14: 49$ on $05-12-92$
Compound Name

Chloromethane

Bromomethane

vinyl Chloride

Chloroethane

Methylene Chloride

Acetone

Carbon Disulfide

1,I-Dichloroethene

1,I-Dichloroethane

1,2-Dichloroethene (total)

trans-1,2-Dichloroethene

Chloroform

1,2-Dichloroethane

2-Butanone

1,1,1-Trichloroethane

Ca'rbon Tetrachloride

Vinyl Acetate

Bromodichloromethane

1,2-Dichloropropane

cis-1, 3-Dichloropropene

Trichloroethene

Dibromochloromethane

I, 1,2-Trichloroethane

Benzene

trans-1,3-Dichloropropene

Bromoform

4-Methyl-2-Pentanone

2-Hexanone

Tetrachloroethene

$1,1,2,2$-Tetrachloroethane

Toluene

Chlorobenzene

Ethylbenzene

styrene

In, $p$-xylene

xylene (total)
CAS Number

$74-87-3$

$74-83-9$

$75-01-4$

$75-00-3$

$75-09-2$

$67-64-1$

$75-15-0$

$75-35-4$

$75-34-3$

540-59-0

540-59-0

$67-66-3$

107-02-2

$78-93-3$

$71-55-6$

56-23-5

108-05-4

$75-27-4$

$78-87-5$

1006I-01-5

79-0I-6

124-48-1

$79-00-5$

$71-43-2$

10061-02-6

75-25-2

108-10-1

591-78-6

127-18-4

79-34-5

108-88-3

108-90-7

$100-41-4$

$100-42-5$

$133-02-7$

$133-02-7$
Result

n.a.

n.d.

n.d.

n.d.

n.d.

64.8

n.d.

n.d.

n.d.

n.d.

n.d.

n.d.

n.d.

54 .

n.d.

n.d.

n.d.

n. a.

n.d.

n.d.

n.d.

n.a.

n.d.

n.d.

n.d.

n.d.

n.d.

n.d.

n.d.

n.d.

75.

n.d.

n.d.

n.d.

n.d.

n.d.

Detection

Units Limit

UG/L 10 .

UG/I 10 .

UG/I 20 .

UG/I 20.

UG/L

UG/L

UG/I

$U G / I$

UG/I

UG/I

UG/I

UG/I

$U G / I$

UG/I

$U G / I$

$U G / L$

$U G / I$

UG/L

UG/I

UG/I

UG/L

UG/L

UG/L

UG/I

UG/I

UG/L

UG/L

UG/I

UG/I

UG/L

UG/I

UG/I

UG/I

UG/L

UG/L

UG/L
5.

10.

5.

5.

5.

5.

5.

5.

5.

10 .

5.

5.

10.

5.

5.

5.

5.

5.

5.

5.

5.

5.

10.

10.

5.

5.

5.

5.

5.

5.

5.

5.

n.d. - not detected

Approved by: 
REECO ANALYTICAL CHEMISTRY LAB'S VOLATILE ORGANICS TËT RESUITS

Name Fleld: 250GS/6-YA W MS

Misc Field: $92-09-048-27 \mathrm{~A}$ MTRX SPK 5.0 MLS

Instrument: VOAI Datafile: >AD797 Idfile: ID_VCl Quantfile: AADT97

Injection time: $17: 56$ on 09-24-92

continuing calibration time : 10:16 on 09-24-92 using the file: AVW320::DI

User dilfac: 1 Quant dilfac: $\quad$ l. Multi-calibration: $14: 49$ on 05-12-92

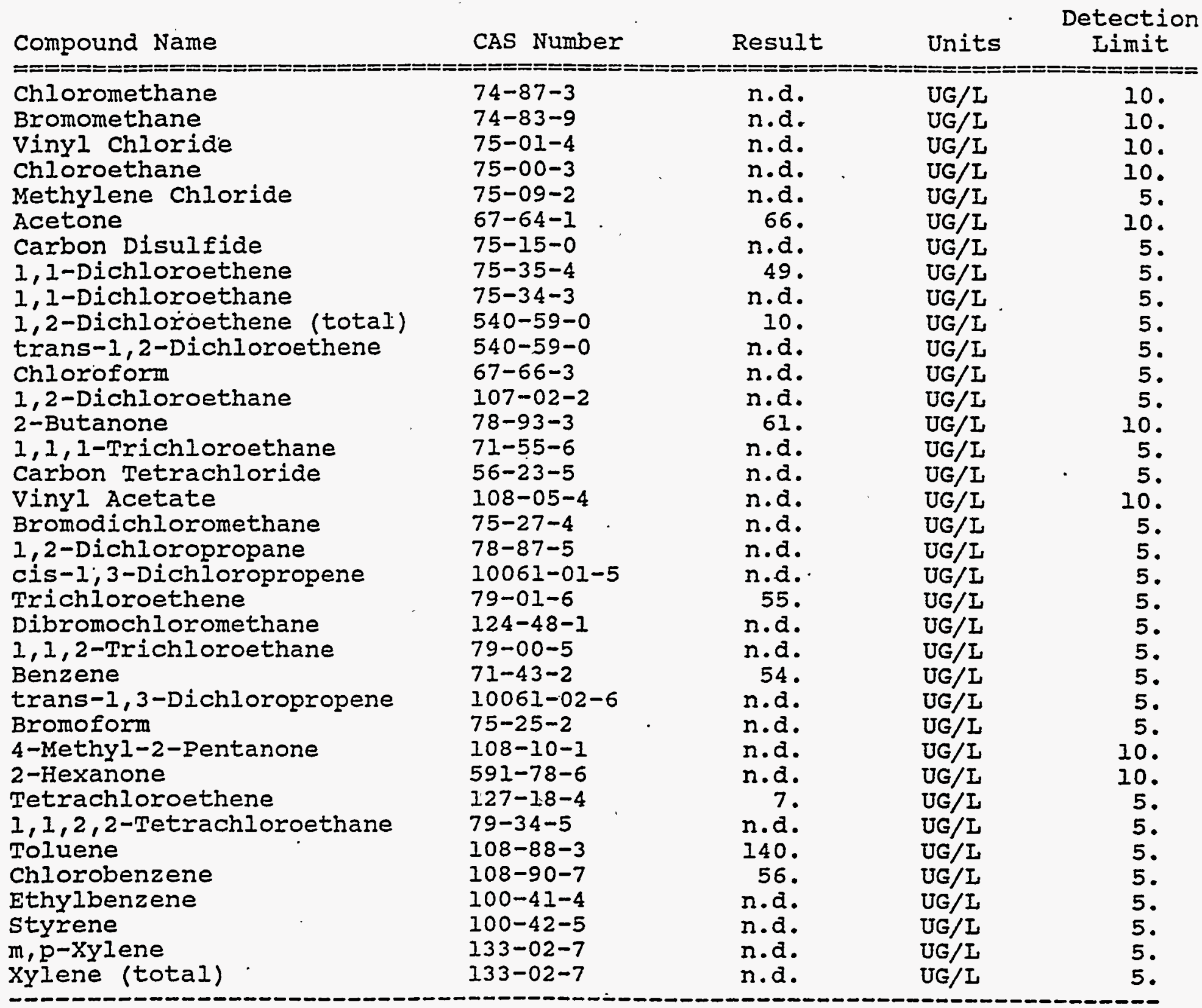

n.a. - not detected

Approved by: 
REECO ANALYTICAL CHEMISTR: LAB'S VOLATILE ORGANICS TEOT RESUITS

Name Field: 250GS/6-YA W MSD

Blank:>VD790:ZERO QAQC File: QVOLWM

Misc Fleld: 92-09-048-28A MTRX SPK DUPL 5.0 MIS

Instrument: VOAI Datafile: >AD798 Idfile:

ID_VCl Quantfile: ^AD798

Injection time: 18:34 on 09-24-92

Continuing calibration time : $10: 16$

User dilfac: 1 Quant dilfac:

on 09-24-92

Quant. Time: 11:04 on 09-25-92 I. Multi-calibration: $14: 49$ on $05-12-92$

Compound Name

CAS Number

Result

Detection

Chloromethane

Bromomethane

Vinyl Chloride

Chloroethane

Methylene Chloride

Acetone

Carbon Disulfide

1,1-Dichloroethene

I, I-Dichloroethane

1,2-Dichloroethene (total)

trans-1,2-Dichloroethene

Chloroform

1,2-Dichloroethane

2-Butanone

1, 1,1-TrichIoroethane

Carbon Tetrachloride

Vinyl Acetate

Bromodichloromethane

1,2-Dichloropropane

cis-1,3-Dichloropropene

Trichloroethene

Dibromochloromethane

1, 1,2-Trichloroethane

Benzene

trans-1,3-Dichloropropene

Bromoform

4-Methy1-2-Pentanone

2-Hexanone

Tetrachloroethene

$1,1,2,2$-Tetrachloroethane

Toluene

Chlorobenzene

Ethylbenzene

styrene

$\mathrm{m}, \mathrm{p}-\mathrm{XY} \mathrm{lene}$

xylene (total)
$74-87-3$

$74-83-9$

$75-01-4$

$75-00-3$

$75-09-2$

$67-64-1$

$75-15-0$

$75-35-4$

$75-34-3$

540-59-0

540-59-0

$67-66-3$

107-02-2

$78-93-3$

$71-55-6$

56-23-5

108-05-4

$75-27-4$

$78-87-5$

$10061-01-5$

79-01-6

124-48-1

$79-00-5$

71-43-2

10061-02-6

75-25-2

108-10-1

.591-78-6

$127-18-4$

$79-34-5$

$108-88-3$

108-90-7

$100-41-4$

$100-42-5$

$133-02-7$

133-02-7 n.d.

n.d.

n.d.

n.d.

n.d.

59.

ก.d.

49.

n.d.

10.

n.d.

n.d.

n.d.

62 .

n.d.

n.d.

n.d.

n.d.

n.d.

n.d.

55 .

n.d.

n.d.

53.

n.d.

n.d.

n.d.

n.d.

7 .

n.d.

120 .

55.

n.d.

n.a.

n.a.

n.d.
Units

UG/I

UG/L

UG/L

UG/I

UG/I

UG/I

UG/I

$U G / I$

$U G / I$

UG/I

$U G / L$

UG/I

UG/I

$U G / I$

UG/I

UG/I

UG/I

$U G / I$

UG/I

$U G / I$

$U G / I$

UG/I

UG/I

UG/I

UG/L

UG/I

UG/I

UG/I

UG/L

$U G / L$

UG/L

UG/I

$U G / I$

$U G / I$

UG/I

$U G / I$
Limit

$=====$

10.

10.

10.

10.

5.

10.

5.

5.

5.

5.

5.

5.

5.

10.

5.

5.

10.

5.

5.

5.

5.

5.

5.

5.

5.

5.

10.

10.

5.

5.

5.

5.

5.

5.

5.

5.

n.d. - not detected

Approved by: 
Name Field: 12-13-1-TK

Misc Field: $92-09-048-20 A$ $0.2 \mathrm{GR} / 10 \mathrm{CC} / / 50$ UL ANALYZED

Instrument: VOAI Datafile: >AD795

Idfile: ID_VCI

QaQC File: QVOLSM

Infection time: $16: 41$ on $09-24-92$

Continuing calibration time : $10: 16$ on $09-24-92$

Quant Time: $17: 13$ on $09-24-92$

User dilfac:5000 Quant dilfac: 5000. Multi-calibration: 14:49 on 05-12-92

Compound Name

$=========$
chloromethane

Bromomethane

Vinyl Chloride

Chloroethane

Methylene Chloride

Acetone

Carbon Disulfide

1,1-Dichloroethene

I,I-Dichloroethane

1,2-Dichloroethene (total)

trans-1,2-Dichloroethene

Chloroform

1,2-Dichloroethane

2-Butanone

1,1,1-Trichloroethane

Carbon Tetrachloride

Vinyl Acetate

Bromodichloromethane

1,2-Dichloropropane

cis-1,3-Dichloropropene

Trichloroethene

Dibromochloromethane

I, 1,2-Trichloroethane

Benzene

trans-1, 3-Dichloropropene

Bromoform

4-Methyl-2-Pentanone

2-Hexanone

Tetrachloroethene

$1,1,2,2$-Tetrachloroethane

Toluene

Chlorobenzene

Ethylbenzene

styrene

m, p-xylene

xylene (total)
CAS Number

$74-87-3$

$74-83-9$

$75-01-4$

$75-00-3$

$75-09-2$

$67-64-1$

$75-15-0$

$75-35-4$

$75-34-3$

540-59-0

540-59-0

$67-66-3$

107-02-2

$78-93-3$

$71-55-6$

$56-23-5$

108-05-4

75-27-4

78-87-5

10061-01-5

79-0I-6

124-48-1

$79-00-5$

$71-43-2$

10061-02-6

75-25-2

108-10-1

$591-78-6$

$127-18-4$

$79-34-5$

$108-88-3$

108-90-7

$100-41-4$

$100-42-5$

133-02-7

133-02-7
Result

n.d.

n.d.

n.d.

n.d.

n.d.

n.d.

n.d.

n.d.

n.d.

n.d.

n.d.

n.d.

n.d.

n.d.

n.d.

n.d.

n.d.

n.d.

$n \cdot d$.

n.d.

n.d.

n.a.

n.d.

110 .

n.d.

n.d.

n.a.

n.d.

n.d.

n.d.

690 .

n.d.

410.

n.d.

1100 .

590 .

Detection

Units Limit

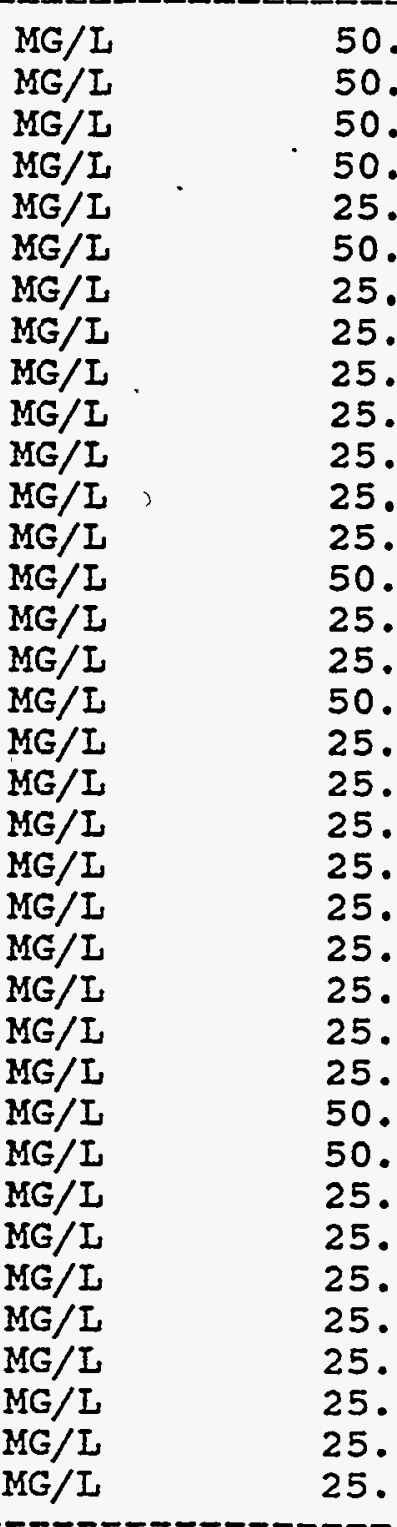

n.d. - not detected

Approved by: 
REECO ANALYTICAL CHEMISIAY LAB'S VOLATIIE ORGANICS TEST RESULTS

Name Field: 12-13-1-TK WATER Blank:>VD790:ZERO QAQC File:QVOLWM Misc Field: 92-09-048-20B 2.5 MLS WATER ANALYZED

Instrument: VOAl Datafile: >AD796 Idfile: ID_VCl Quantfile: ^AD796

Injection time: $17: 19$ on $09-24-92$

Continuing calibration time : $10: 16$ on $09-24-92$ using the file: ^VW320::DI

User dilfac:2 Quant dilfac:2

Multi-calibration: $14: 49$ on $05-12-92$

\begin{tabular}{|c|c|c|c|c|}
\hline 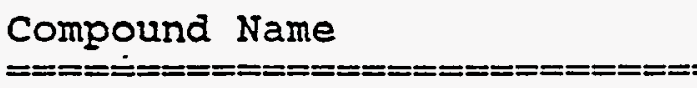 & $\begin{array}{l}\text { CAS Number } \\
==========\end{array}$ & Result & $\begin{array}{l}\text { Units } \\
=======\end{array}$ & Limit \\
\hline 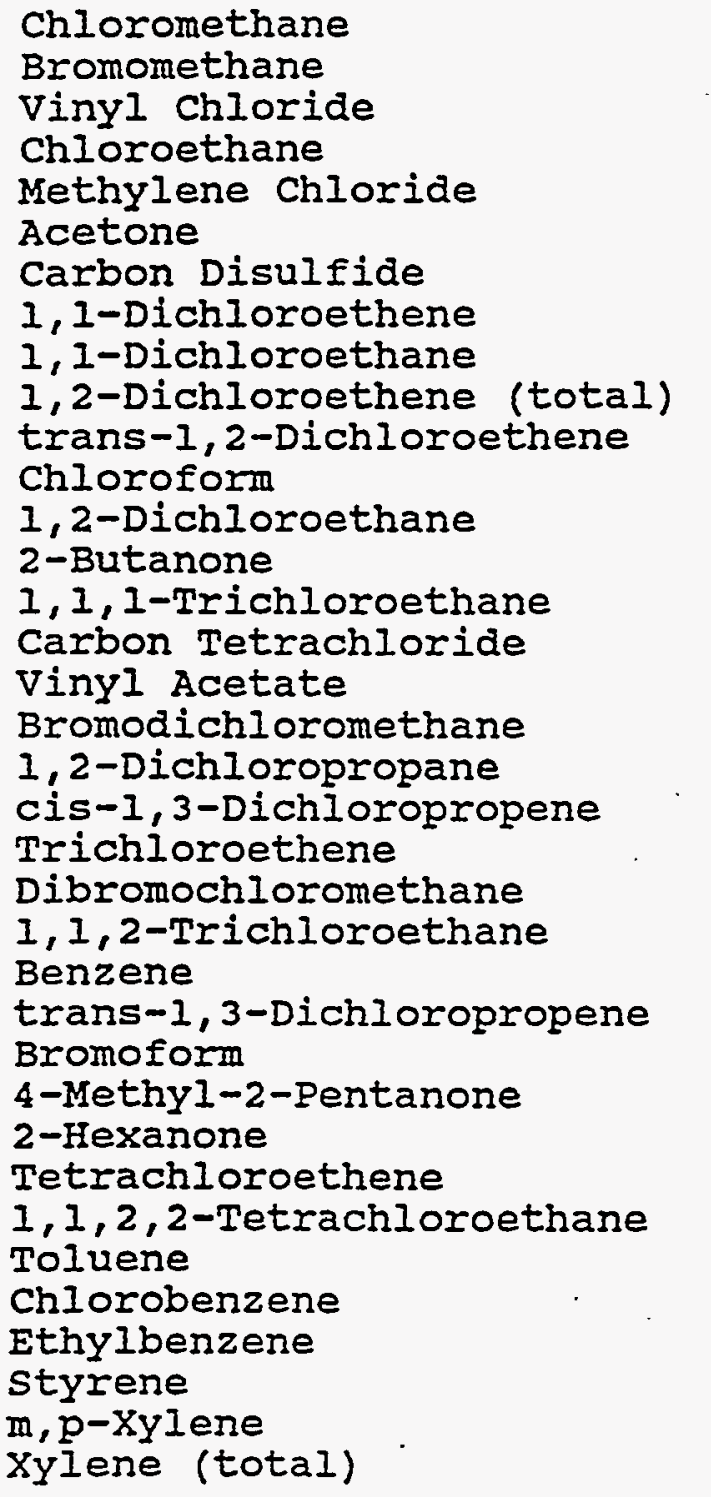 & 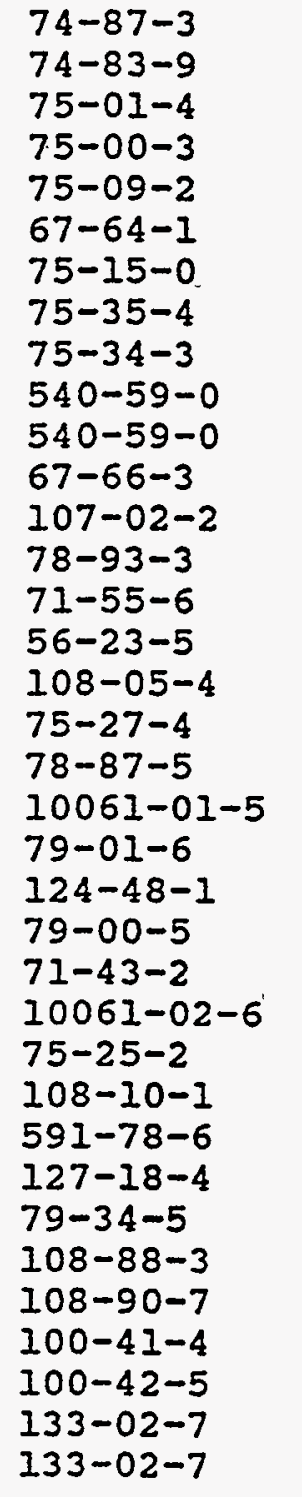 & 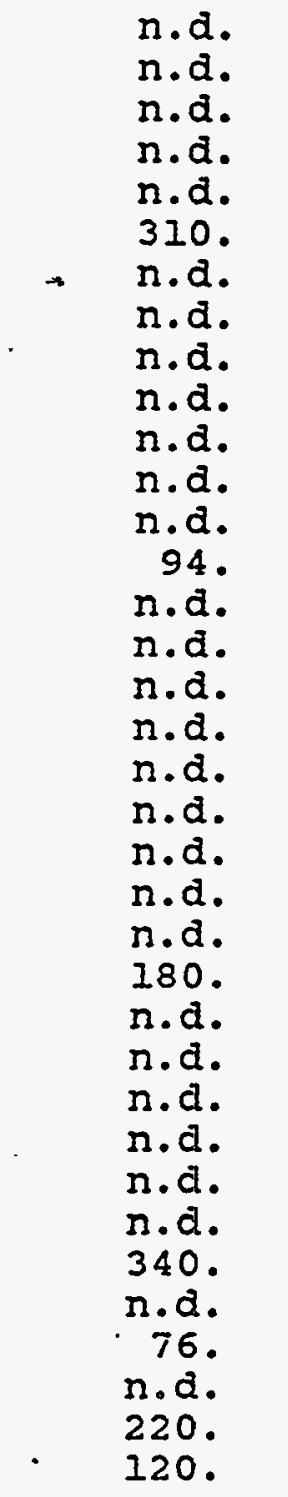 & $\begin{array}{l}U G / I \\
U G / I \\
U G / I \\
U G / L \\
U G / I \\
U G / I \\
U G / I \\
U G / I \\
U G / I \\
U G / I \\
U G / I \\
U G / I \\
U G / I \\
U G / I \\
U G / I \\
U G / I \\
U G / I \\
U G / I \\
U G / I \\
U G / I \\
U G / I \\
U G / I \\
U G / I \\
U G / I \\
U G / I \\
U G / I \\
U G / I \\
U G / I \\
U G / I \\
U G / I \\
U G / I \\
U G / I \\
U G / L \\
U G / I \\
U G / I \\
U G / I\end{array}$ & $\begin{array}{l}20 . \\
20 . \\
20 . \\
20 . \\
10 . \\
20 . \\
10 . \\
10 . \\
10 . \\
10 . \\
10 . \\
10 . \\
10 . \\
20 . \\
10 . \\
10 . \\
20 . \\
10 . \\
10 . \\
10 . \\
10 . \\
10 . \\
10 . \\
10 . \\
10 . \\
10 . \\
20 . \\
20 . \\
10 . \\
10 . \\
10 . \\
10 . \\
10 . \\
10 . \\
10 . \\
10 .\end{array}$ \\
\hline
\end{tabular}

n.d. - not detected

Approved by: 
REECO ANALYTICAI CHEMISTR I LAB'S VOLATILE ORGANICS TEOT RESULTS

Name Field: 92SABO916-1 TRPBIK

Blank:>VD784:PASS QaQC File: QVOLWM.

M1sc Fleld: $92-09-048-25 A$

Instrument: VOAI Datafile: >VD785

Idfile: ID_VCl Quantfile: ^VDi85

Injection time: $13: 44$ on $09-23-92$

Continuing calibration time : 08:19 on 09-23-92 using the file: AVW318::DI

User dilfac: 1 Quant dilfac: 1. Multi-calibration: 14:49 on 05-12-92

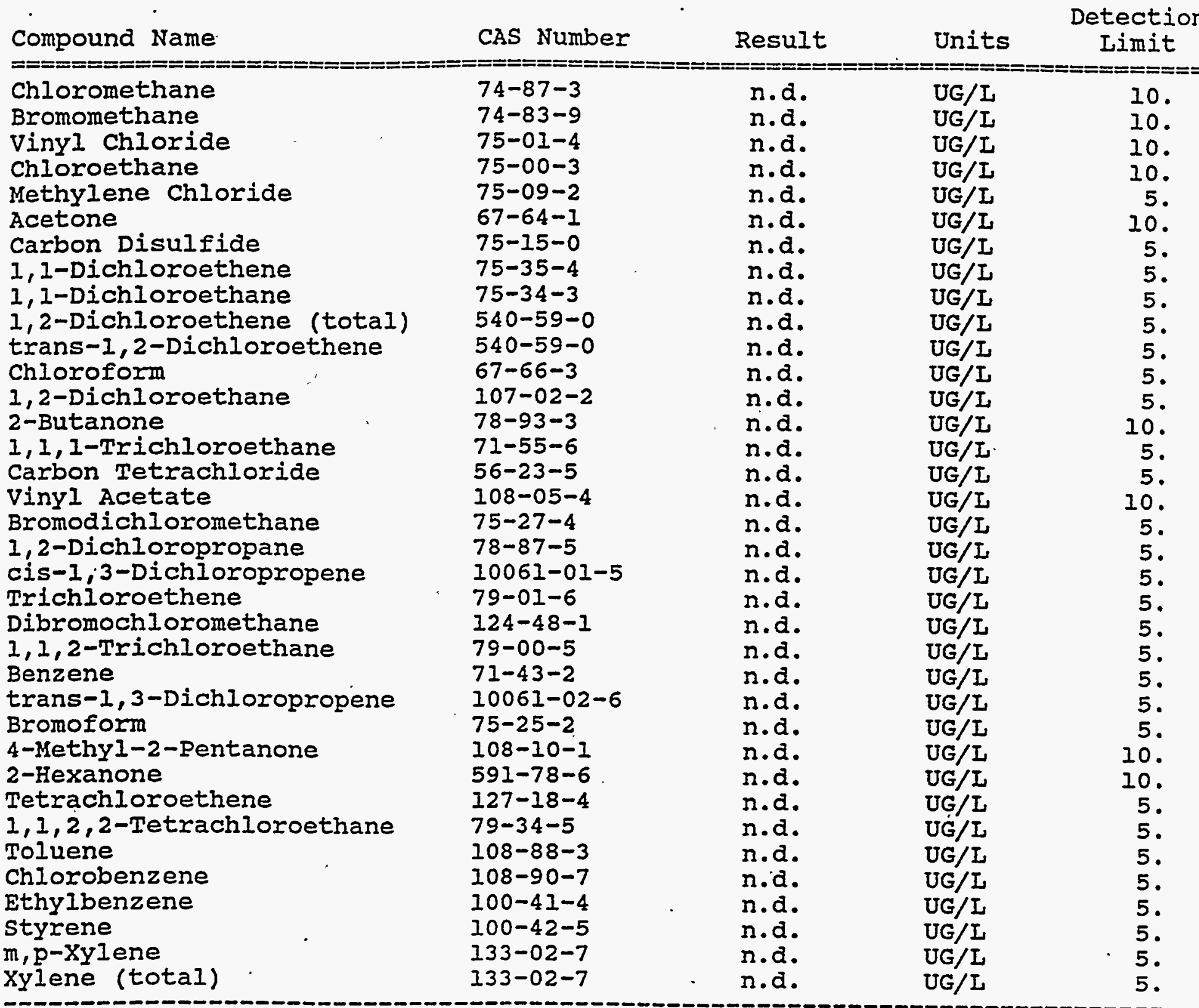

n.d. - not detected

Approved by: 
Blank Run Report REECO ANALYTICAL CHEMISTRY LAB'S VOLATILE ORGANICS TEST RESULTS

Name Field: VBLKOI

Misc Field: METHOD BLANK

Instrument: VOAI
Datafile: $>$ VD784

QaQC File: QVOLWM

Injection time: $12: 35$ on $09-23-92$

continuing calibration time : 08:19

User dilfac: 1

Quant dilfac:

on $09-23-92$

Idfile: ID_VCl Quantfile: ^VD784

Quant Time: $23: 37$ on $09-23-92$

1. Wulti-calibing the file: ^Vw318::D1

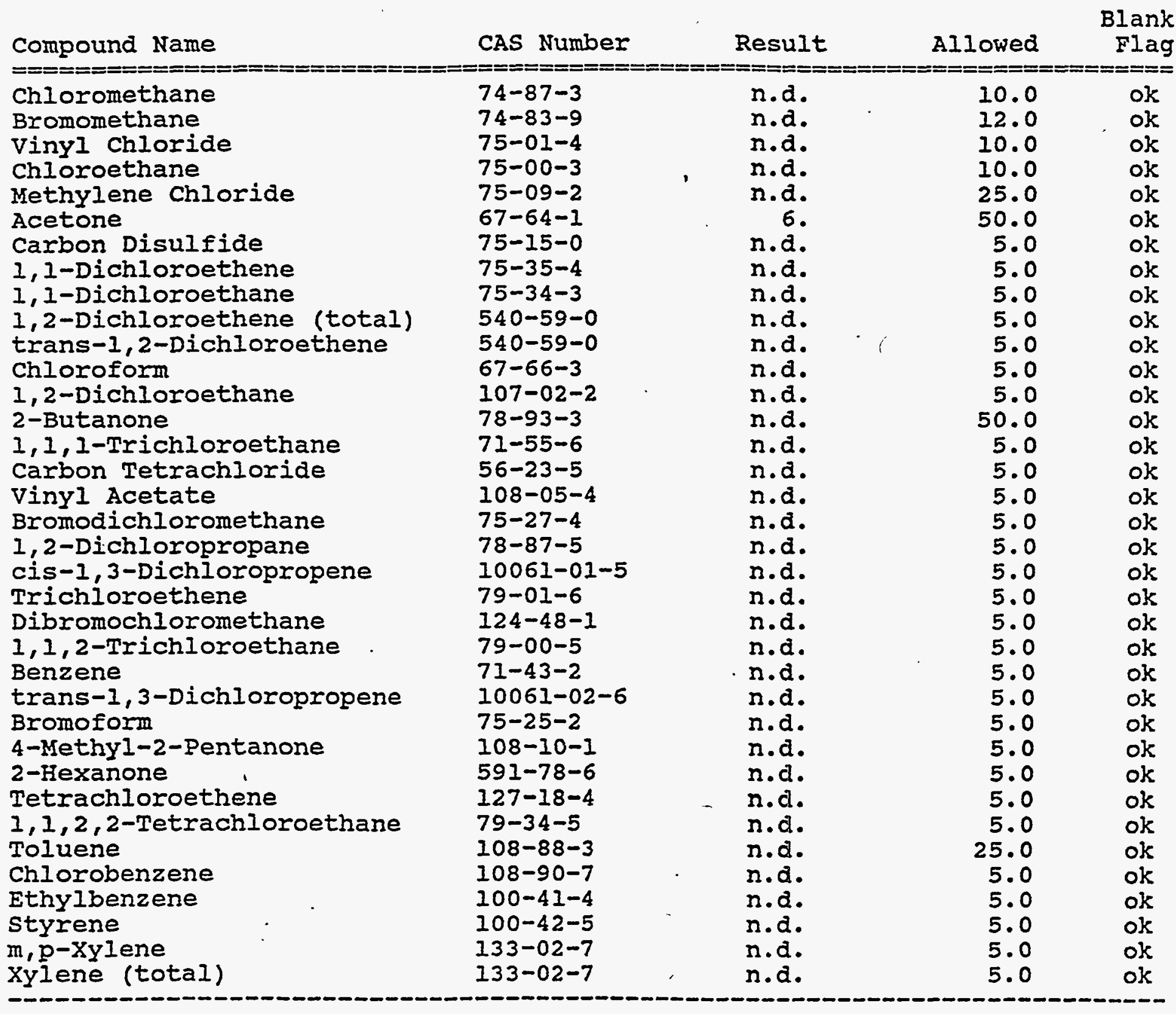

n.d. - not detected

Approved by: 
Blank Run Report

REECO ANALYTICAL CHEMISTRY LAB'S VOLATIIE ORGANICS TEST RESULTS

Name Fleld: VBIKOI

Misc Fleld: METHOD BLANK

Instrument: VOAI Datafile: >VD790

Idfile: ID_VCI Quantfile: ^VD790

Injection time: 11:04 on 09-24-92

Continuing calibration time: $10: 16$ on $09-24-92$ using the file: $\wedge$ VW $320::$ DI

User dilfac: I

Quant dilfac:

1. Multi-calibration: $14: 49$ on 05-12-92

\begin{tabular}{|c|c|c|c|c|}
\hline 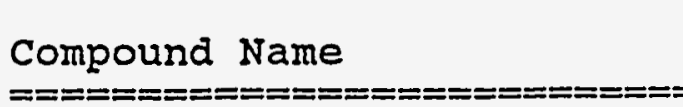 & $\begin{array}{l}\text { CAS Number } \\
==========\end{array}$ & Result & Allowed & $\begin{array}{r}\text { Blank } \\
\text { Flag }\end{array}$ \\
\hline 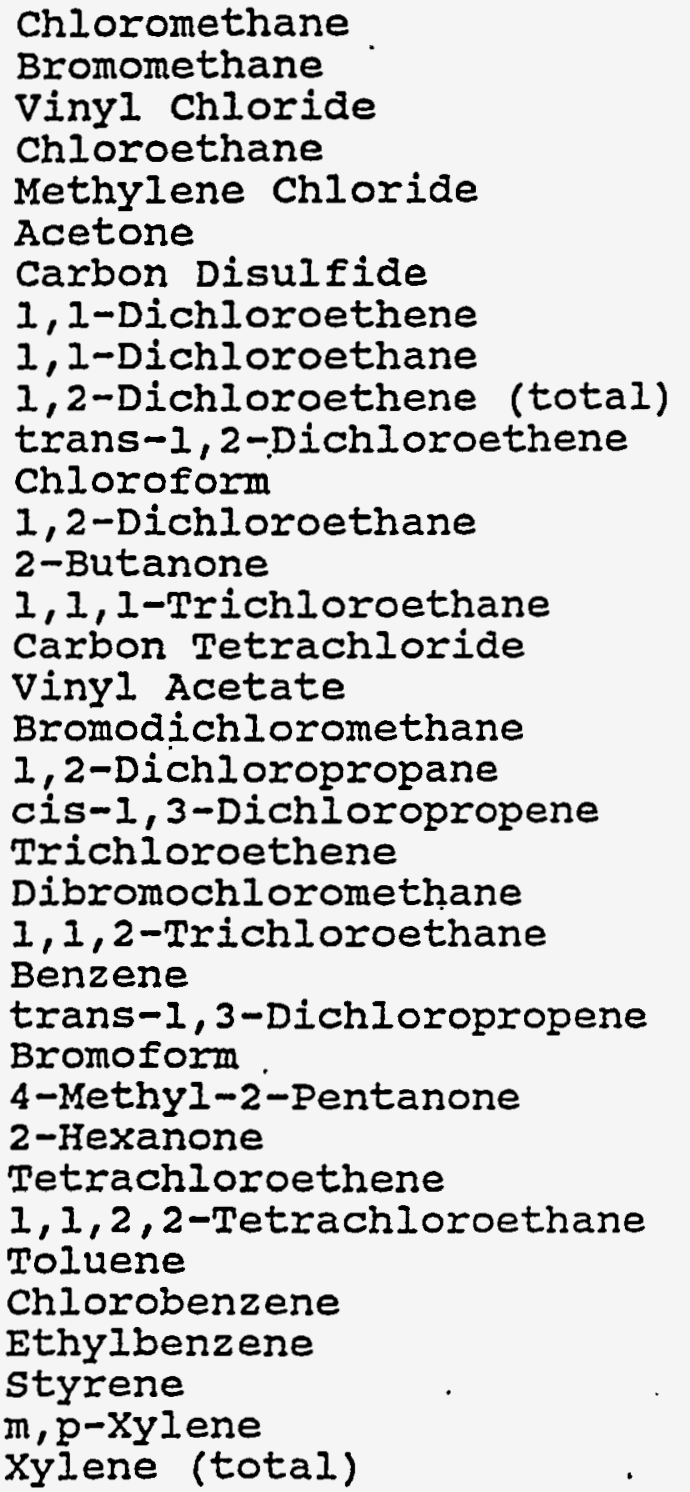 & 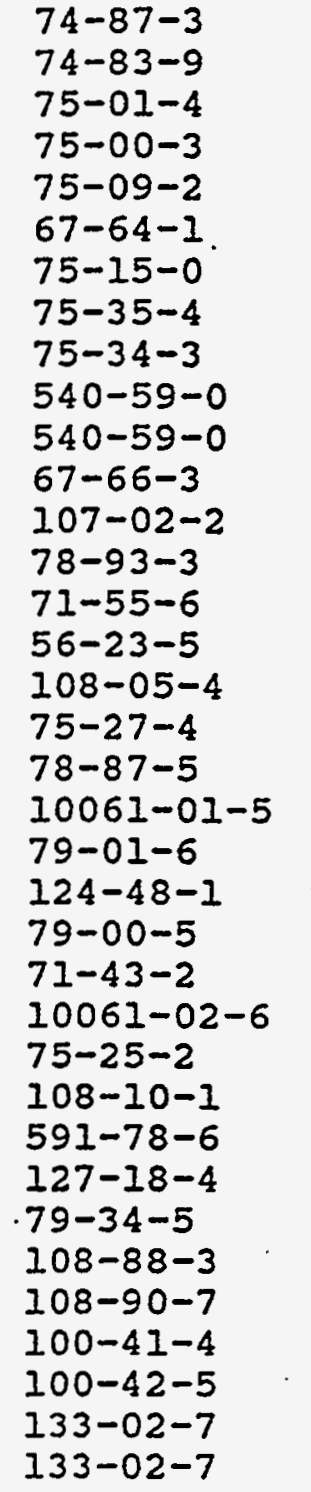 & 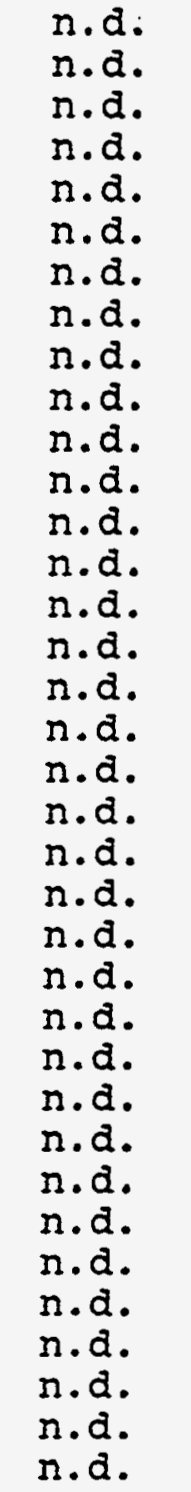 & $\begin{array}{r}10.0 \\
12.0 \\
10.0 \\
10.0 \\
25.0 \\
50.0 \\
5.0 \\
5.0 \\
5.0 \\
5.0 \\
5.0 \\
5.0 \\
5.0 \\
50.0 \\
5.0 \\
5.0 \\
5.0 \\
5.0 \\
5.0 \\
5.0 \\
5.0 \\
5.0 \\
5.0 \\
5.0 \\
5.0 \\
5.0 \\
5.0 \\
5.0 \\
5.0 \\
5.0 \\
5.0 \\
25.0 \\
5.0 \\
5.0 \\
5.0 \\
5.0 \\
5.0\end{array}$ & $\begin{array}{l}\text { ok } \\
\text { ok } \\
\text { ok. } \\
\text { ok } \\
\text { ok } \\
\text { ok } \\
\text { ok } \\
\text { ok } \\
\text { ok } \\
\text { ok } \\
\text { ok } \\
\text { ok } \\
\text { ok } \\
\text { ok } \\
\text { ok } \\
\text { ok } \\
\text { ok } \\
\text { ok } \\
\text { ok } \\
\text { ok } \\
\text { ok } \\
\text { ok } \\
\text { ok } \\
\text { ok } \\
\text { ok } \\
\text { ok } \\
\text { ok } \\
\text { ok } \\
\text { ok } \\
\text { ok } \\
\text { ok } \\
\text { ok } \\
\text { ok } \\
\text { ok } \\
\text { ok } \\
\text { ok }\end{array}$ \\
\hline
\end{tabular}

n.d. - not detected

Approved by: 
..
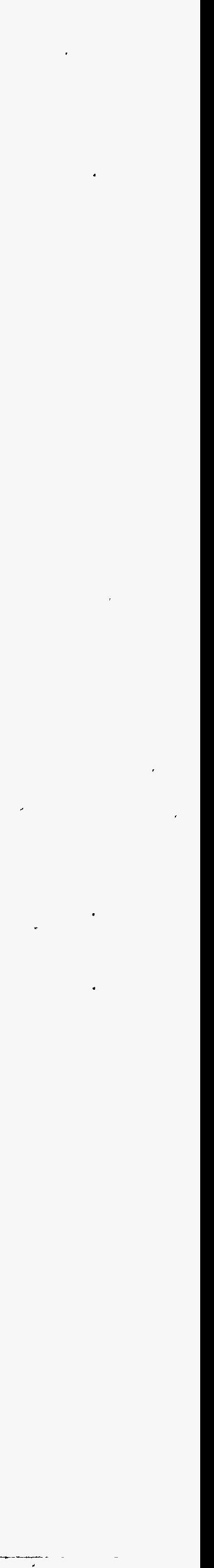
SEMIVOLATILES DATA 

ANALYST NOTES: BNAS

Date: October, 12, 1992

Lab Work Order No.: 92-09-048

Client Sample ID: 11-1-1-TK water, 12-13-1-TK water, SBLK 0921, 11-1-1TK oil, 12-13-1-TK oil, 12-13-1-TK HS, 12-13-1-TK HSD

The samples were analyzed on a Hewlett Packard 5890 GC/5988. Data were collected on an HP1000/RTE-A data system. Instrumental parameters used are those specified in SW-846 method 8270 .

The target analytes are listed with their CAS numbers, and the amount of the target compound found' in $\mathrm{ug} / \mathrm{L}$ or $\mathrm{ug} / \mathrm{Kg}$ depending on the matrix. If the analyte was not found in the sample, the quantitation limit is reported followed by an $n . d$. qualifier. The other qualifiers used in this reporting format are:

n.d.-Indicates compound was analyzed for but not detected.

$J$ - Indicates an estimated value. There is a compound present which meets the identification criteria, but the result is less than the sample quantitation limit and greater than zero.

B- This flag is used when the analyte is found in the associated blank as well as in the sample. It indicates possible/probable blank contamination.

E- This flag identifies compounds whose concentrations exceed the calibration range of the GC/MS instrument for that specific analysis.

D- This flag identifies all compounds identified in an analysis at a secondary dilution factor.

The four oil samples had no problems during analysis. However, the water samples showed serious matrix effects with most of the surrogates and internal standards failing $Q A / Q C$. 
REECO ANALYTICAL CIEMISTRY LAB'S SEMIVOLATILE ORGANIC: TEST RESULTS EPA METHOD:625/8270 LAB SOPS:F.D.05.01\& F.D.03.02

- Page - 1

Name Field: 11-1-1-TK OIL

Operator: KRAIG

Misc Field: $9209048-01 A$

Instrument: BNA1 Datafile: >SD115

Idfile: ID_BC1 Quantfile: ^SD115

Injection time: $14: 21$ on 10-07-92

Continuing calibration time : $12: 04$ on 10-07-92

Quant Time: $15: 12$ on 10-07-92 User dilfac:44.2 Quant dilfac:

1. Multi-calibra

using the file: ASC154::D4

Compound Name

CAS Number

Result

Detection

Phenol

bis (2-chloroethyl) ether

2-Chlorophenol

1,3-Dichlorobenzene

1,4-Dichlorobenzene

Benzyl alcohol

1,2-Dichlorobenzene

2-Methylphenol

2,2'-oxybis (1-Chloropropane)

bis (2-Chloroisopropyl) ether

4-Methylphenol

$\mathrm{N}$-Nitroso-di-n-propylamine

Hexachloroethane

Nitrobenzene

Isophorone

2-Nitrophenol

2,4-Dimethylphenol

Benzoic acid

bis (2-Chloroethoxy) methane

2,4-Dichlorophenol

1,2,4-Trichlorobenzene

Naphthalene

4-Chloroaniline

Hexachlorobutadiene

4-Chloro-3-methylphenol

2-Methylnaphthalene

Hexachlorocyclopentadiene

$2,4,6$-Trichlorophenol

$2,4,5$-Trichlorophenol

2-ChIoronaphthalene

2-Nitroaniline

Dimethylphthalate

Acenaphthylene

2,6-Dinitrotoluene

3-Nitroaniline

Acenaphthene

2, 4-Dinitrophenol

4-Nitrophenol

Dibenzofuran

2,4-Dinitrotoluene

Diethylphthalate

4-Chlorophenyl-phenylether

Fluorene

4-Nitroaniline

108-95-2

111-44-4

95-57-8

541-73-1

106-46-7

100-51-6

95-50-1

95-48-7

108-60-1

$39638-32-9$

106-44-5

621-64-7

$67-72-1$

$98-95-3$

$78-59-1$

$88-75-5$

$105-67-9$

65-85-0

111-91-1

120-83-2

$120-82-1$

91-20-3

$106-47-8$

$87-68-3$

59-50-7

$91-57-6$

$77-47-4$

88-06-2

95-95-4

91-58-7

$88-74-4$

$131-11-3$

208-96-8

606-20-2

99-09-2

$83-32-9$

51-28-5

100-02-7

132-64-9

121-14-2

84-66-2

7005-72-3

$86-73-7$

n.d.

Units

Iimit

100-01-6

n.d.

n.d.

n.d.

n.d.

n.d.

n.d.

n.d.

n.d.

n.d.

n.d.

n.d.

n.d.

n.d.

n.d.

n.d.

n.d.

n.d.

n.d.

n.d.

n.d.

n.d.

n.d.

n.d.

n.d.

1340 .

n.d.

n.d.

n.d.

n.d.

n.d.

n.d.

n.d.

n.d.

n.d.

n.d.

n.d.

n.d.

n.d.

n.d.

n.d.

n.d.

n.d.

n.d.

$\mathrm{ug} / \mathrm{I}$

ug/L

$u g / L$

ug/L

$\mathrm{ug} / \mathrm{L} 220$.

$\mathrm{ug} / \mathrm{I} 220$.

$\mathrm{ug} / \mathrm{I} 220$.

$\mathrm{ug} / \mathrm{L} 220$.

$\mathrm{ug} / \mathrm{L} 220$.

$\mathrm{ug} / \mathrm{I} 220$.

$\mathrm{ug} / \mathrm{L} 220$.

$\mathrm{ug} / \mathrm{I} \quad 220$.

ug/I 220 .

$\mathrm{ug} / \mathrm{L} \quad 220$.

$\mathrm{ug} / \mathrm{I} 220$.

$\mathrm{ug} / \mathrm{L} .220$.

$\mathrm{ug} / \mathrm{I} \quad 220$.

$\mathrm{ug} / \mathrm{L} \quad 1100$.

$\mathrm{ug} / \mathrm{L} 220$.

$\mathrm{ug} / \mathrm{I} \quad 220$.

$\mathrm{ug} / \mathrm{I} 220$.

$\mathrm{ug} / \mathrm{I} \quad 220$.

$\mathrm{ug} / \mathrm{I}$

220 .

$\mathrm{ug} / \mathrm{L}$

220 .

$\mathrm{ug} / \mathrm{I} \quad 220$.

$\mathrm{ug} / \mathrm{L} 220$.

$\mathrm{ug} / \mathrm{L} 220$.

$\mathrm{ug} / \mathrm{L} 220$.

$\mathrm{ug} / \mathrm{L} \quad 1100$.

$\mathrm{ug} / \mathrm{L}$

220.

$u g / L$

$\mathrm{ug} / \mathrm{L}$

1100 .

$\mathrm{ug} / \mathrm{I}$

$\mathrm{ug} / \mathrm{I}$

$u g / L$

$\mathrm{ug} / \mathrm{L}$

$\mathrm{ug} / \mathrm{L}$

$\mathrm{ug} / \mathrm{L}$

$\mathrm{ug} / \mathrm{I}$

$\mathrm{ug} / \mathrm{L}$

$\mathrm{ug} / \mathrm{L}$

$u g / L$

220.

220 .

220.

1100 .

220.

1100 .

1100 .

220 .

220 .

220 .

220 .

220 .

$\mathrm{ug} / \mathrm{L}$

1100 . 
REECO ANALYTICAL CHEMISTRY IAB'S SEMIVOLATILE ORGANICS TEST RESULTS EPA METHOD:625/8270 LAB SOPS:F.D.05.01\& F.D.03.02.

- Page - 2

Name Field: $11-1-1-$ TK OIL

Operator: KRAIG

Misc Field: $9209048-01 \mathrm{~A}$

Instrument: BNAI Datafile: >SD115 Idfile: ID_BCI Quantfile: ^SDI15

Compound Name

CAS Number

Result

Detectio

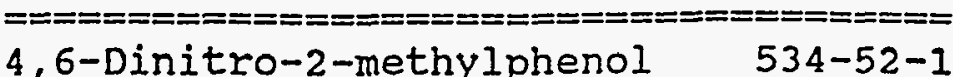

$\mathrm{N}-\mathrm{N}$ itrosodiphenylamine

4-Bromophenyl-phenylether

$86-30-6$

$101-55-3$

Hexachlorobenzene

Pentachlorophenol

$118-74-1$

$87-86-5$

Phenanthrene

$85-01-8$

Anthracene

$120-12-7$

Carbazole

$86-74-8$

Di-n-butylphthalate

$84-74-2$

Fluoranthene

206-44-0

Pyrene

129-00-0

Butylbenzylphthalate

$3,3^{\prime}$-Dichlorobenzidine

$85-68-7$

Benzo (a) anthracene

$91-94-1$

$56-55-3$

Chrysene

218-01-9

bis (2-Ethylhexyl) phthalate

$117-81-7$

117-84-0

205-99-2

Benzo(b) fluoranthene

207-08-9

Benzo (k) fluoranthene

$50-32-8$

Indeno $(1,2,3-c d)$ pyrene

$193-39-5$

Dibenzo $(a, h)$ anthracene

$53-70-3$

Benzo $(g, h, i)$ perylene

191-24-2

n. $d$.

n.d.

n.d.

n.d.

n.d.

n.d.

n.d.

n.d.

n.d.

n.d.

n.d.

n.d.

n.d.

n.d.

n.d.

n.d.

n.d.

n.d.

n.d.

n.d.

n.d.

n.d.

n.d.
Iimit

Units .

$====$

\begin{tabular}{|c|c|}
\hline $\mathrm{ug} / \mathrm{L}$ & 1100. \\
\hline $\mathrm{ug} / \mathrm{L}$ & 220. \\
\hline$u g / L$ & 220. \\
\hline $\mathrm{ug} / \mathrm{L}$ & 220. \\
\hline$u g / L$ & 1100. \\
\hline$u g / L$ & 220. \\
\hline$u g / L$ & 220 \\
\hline$u g / I$ & 220 \\
\hline $\mathrm{ug} / \mathrm{L}$ & 220 \\
\hline $\mathrm{ug} / \mathrm{L}$ & 220. \\
\hline $\mathrm{ug} / \mathrm{L}$ & 220. \\
\hline$u g / L$ & 220 . \\
\hline$u g / I$ & 440 \\
\hline ug/L & 220. \\
\hline$u g / I$ & 220. \\
\hline$u g / I$ & 220 \\
\hline$u g / L$ & 220 \\
\hline $\mathrm{ug} / \mathrm{L}$ & 220 \\
\hline $\mathrm{ug} / \mathrm{L}$ & 220 \\
\hline $\mathrm{ug} / \mathrm{L}$ & 220 \\
\hline ug/I & 220 \\
\hline ug/L & 220 \\
\hline $\mathrm{ug} / \mathrm{I}$ & 220 \\
\hline
\end{tabular}

n.d. - not detected

Approved by: 
REECO ANALYTICAL CHEMISTRY LAB'S SEMIVOLATILE ORGANICS TEST RESULTS

EPA METHOD: $625 / 8270$ LAB SOPS:F.D.05.01 \& F.D.03.02

- Page - 1

Name Field: 11-1-1-TK WATER

Misc Field: 9209048 -01B 2ND RUN

Instrument: BNA2 Datafile: >SD275

Idfile: ID_BC2 Quantflle: ^SD275

Injection time: $18: 49$ on 10-07-92

Continuing calibration time : $16: 44$ on 10-07-92 using the file: ^sC243::D5

User dilfac: 21 Quant dilfac:

1. Multi-calibration: $15: 20$ on $06-24-92$

\begin{tabular}{|c|c|c|c|c|}
\hline Compound Name & $\begin{array}{l}\text { CAS Number } \\
===========\end{array}$ & $\begin{array}{l}\text { Result } \\
========\end{array}$ & Units & $\begin{array}{l}\text { Letecti } \\
\text { Limit }\end{array}$ \\
\hline 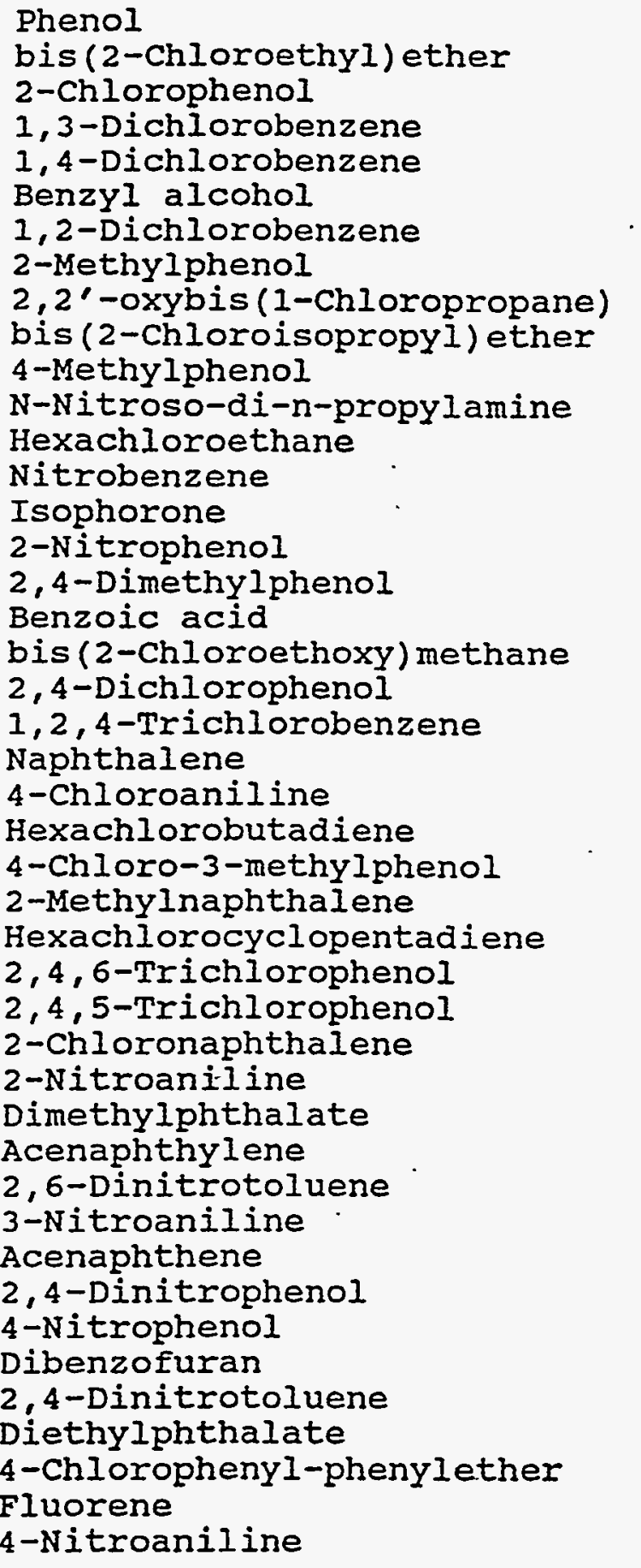 & 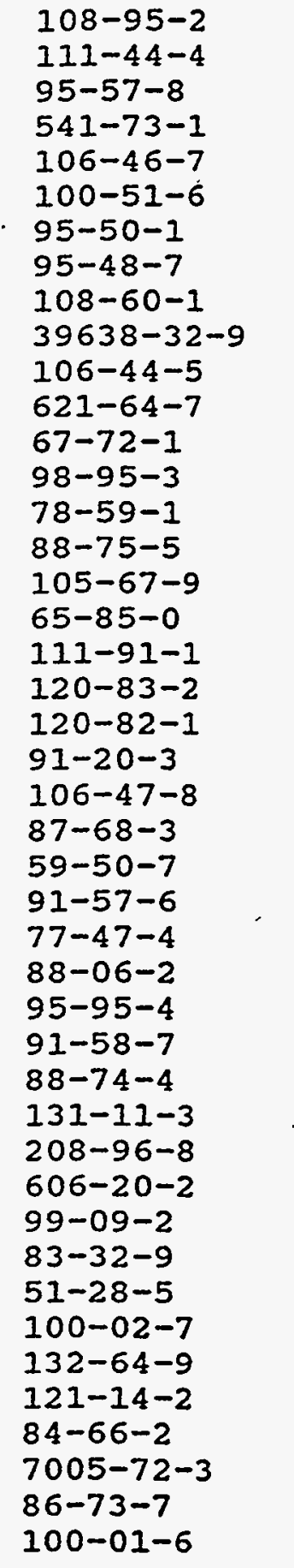 & 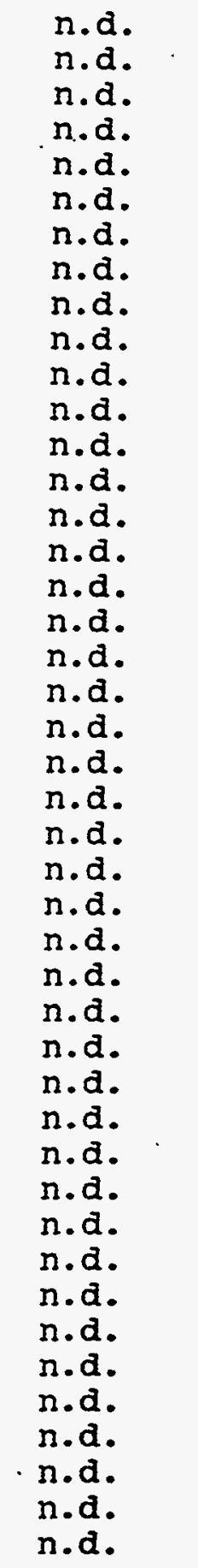 & 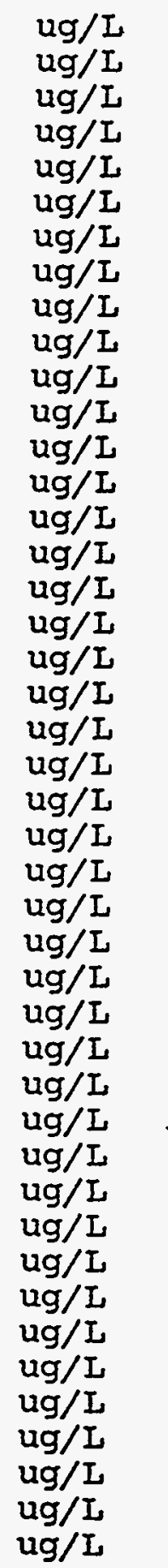 & $\begin{array}{l}110 . \\
110 . \\
110 . \\
110 . \\
110 . \\
110 . \\
110 . \\
110 . \\
110 . \\
530 . \\
110 . \\
110 . \\
110 . \\
110 . \\
110 . \\
110 . \\
110 . \\
110 . \\
110 . \\
110 . \\
530 . \\
110 . \\
530 . \\
110 . \\
110 . \\
110 . \\
530 . \\
110 . \\
530 . \\
530 . \\
110 . \\
110 . \\
110 . \\
110 . \\
110 . \\
530 .\end{array}$ \\
\hline
\end{tabular}


REECO ANALYTICAL CHEMISTRY LAB'S SEMIVOLATILE ORGANICS TEST RESULTS EPA METHOD: 625/8270 LAB SOPS:F.D.05.01\& F.D.03.02

- Page - 2

Name Field: 11-1-1-TK WATER

Operator: KRAIG

Misc Field: $9209048-01 B$ 2ND RUN

Instrument: BNA2 Datafile: >SD275 Idfile: ID_BC2 Quantfile: ^SD275

Compound Name

CAS Number

Result

Units

Detectio

$=====================$

$\mathrm{N}$-Nitrosodiphenylamine

$534-52-1$

4-Bromophenyl-phenylether

$86-30-6$

Hexachlorobenzene

Pentachlorophenol

$101-55-3$

$118-74-1$

Phenanthrene

$87-86-5$

Anthracene

$85-01-8$

Carbazole

$120-12-7$

$86-74-8$

$84-74-2$

206-44-0

129-00-0

$85-68-7$

$91-94-1$

$56-55-3$

218-01-9

$117-81-7$

$117-84-0$

n.d.

n.d.

n.d.

n.d.

n.d.

n.d.

n.d.

n.d.

n. d.

n.d.

n.d.

n.d.

n.d.

n.d.

Chrysene

n.d.

n.d.

205-99-2

n.d.

Di-n-octyiphthalate

Benzo (b) fiuoranthene

207-08-9

n.d.

$50-32-8$

Benzo (a) pyrene

Indeno $(1,2,3-c d)$ pyrene

Dibenzo $(a, h)$ anthracene

$193-39-5$

$53-70-3$

Benzo $(g, h, i)$ perylene

191-24-2

n.d.

n.d.

n.d.

n.d.

n.d.

$\mathrm{ug} / \mathrm{I}$

Iimit

n.d. - not detected

Approved by: 
REECO ANALYTICAL CHEMIS LRY LAB'S SEMIVOLATILE ORGANICS TEST RESULTS EPA METHOD: $625 / 8270$ LAB SOPS:F.D.05.01\& F.D.03.02

- Page - 1

Name Field: 12-13-1-TK OIL

Operator: KRAIG

Misc Field: $9209048-18 \mathrm{~A}$

Instrument: BNAI . Datafile: >SD116

Idfile: ID_BCl Quantfile: ^SD116

Injection time: $15: 23$ on 10-07-92

Continuing calibration time: $12: 04$ on 10-07-92 using the file: ^SC154::D4

User dilfac:45.9 Quant dilfac: 1. Multi-calibration: 10:28 on 09-22-92

Compound Name

Cạs Number

Result

Detection

Phenol

bis (2-Chloroethyl) ether

2-Chlorophenol

1,3-Dichlorobenzene

1,4-Dichlorobenzene

Benzyl alcohol

1,2-Dichlorobenzene

2-Methylphenol

2,2'-oxybis (1-Chloropropane)

bis (2-Chloroisopropyl) ether

4-Methylphenol

$\mathrm{N}-\mathrm{Nitroso-di-n-propylamine}$

Hexachloroethane

Nitrobenzene

Isophorone

2-Nitrophenol

2,4-Dimethylphenol

Benzoic acid

bis (2-Chloroethoxy) methane

2,4-Dichlorophenol

1,2,4-Trichlorobenzene

Naphthalene

4-chloroaniline

Hexachlorobutadiene

4-Chloro-3-methylphenol

2-Methylnaphthalene

Hexachlorocyclopentadiene

$2,4,6$-Trichlorophenol

$2,4,5$-Trichlorophenol

$108-95-2$

$111-44-4$

95-57-8

$541-73-1$

106-46-7

$100-51-6$

95-50-1

95-48-7

$108-60-1$

$39638-32-9$

$106-44-5$

621-64-7

67-72-1

98-95-3

$78-59-1$

$88-75-5$

105-67-9

65-85-0

$111-91-1$

$120-83-2$

$120-82-1$

91-20-3

$106-47-8$

$87-68-3$

59-50-7

91-57-6

$77-47-4$

88-06-2

95-95-4

91-58-7

$88-74-4$

2-Nitroaniline

Dimethylphthalate

Acenaphthylene

2,6-Dinitrotoluene

3-Nitroaniline

Acenaphthene

2,4-Dinitrophenol

4-Nitrophenol

Dibenzofuran

2,4-Dinitrotoluene

Diethylphthalate

4-Chlorophenyl-phenylether

Fluorene

4-Nitroaniline
131-11-3

208-96-8

$606-20-2$

99-09-2

83-32-9

51-28-5

100-02-7

$132-64-9$

$121-14-2$

84-66-2

$7005-72-3$

86-73-7

100-01-6 n.d.

n.d.

n.d.

n.d.

n.d.

n.d.

n.d.

n.d.

n.d.

n.d.

n.d.

n.d.

n.d.

n.d.

n.d.

n.d.

n.d.

n.d.

n.d.

n.d.

n.d.

n.d.

n.d.

n.d.

n.d.

1000.

n.d.

n.a.

n.d.

n.d.

$n: d$.

n.d.

n.d.

n.d.

n.d.

n.d.

n.d.

n.d.

n.d.

n.d.

n.d.

n. a.

n.d.

n.d.
Units

$\mathrm{ug} / \mathrm{I}$

ug/L

ug/L

$u g / L$

ug/L

ug/L

ug/L

$u g / L$

ug/L

ug/L

ug/I

ug/L

ug/L

ug/L

$u g / L$

$u g / I$

$\mathrm{ug} / \mathrm{L}$

$\mathrm{ug} / \mathrm{I}$

$u g / L$

ug/L

$\mathrm{ug} / \mathrm{L}$

$\mathrm{ug} / \mathrm{I}$

$\mathrm{ug} / \mathrm{L}$

$\mathrm{ug} / \mathrm{L}$

$\mathrm{ug} / \mathrm{I}$

$\mathrm{ug} / \mathrm{L}$

$\mathrm{ug} / \mathrm{I}$

$\mathrm{ug} / \mathrm{L}$

$\mathrm{ug} / \mathrm{I}$

$\mathrm{ug} / \mathrm{I}$

ug/I

$u g / L$

$\mathrm{ug} / \mathrm{I}$

$\mathrm{ug} / \mathrm{L}$

$\mathrm{ug} / \mathrm{L}$

$\mathrm{ug} / \mathrm{I}$

$\mathrm{ug} / \mathrm{I}$

$\mathrm{ug} / \mathrm{L}$

$\mathrm{ug} / \mathrm{L}$

$\mathrm{ug} / \mathrm{I}$

$\mathrm{ug} / \mathrm{L}$

ug/L

$\mathrm{ug} / \mathrm{I}$

$\mathrm{ug} / \mathrm{L}$
Limit 
REECO ANALYTICAL CHEMISTRY LAB'S SEMIVOLATILE ORGANICS TEST RESULTS EPA METHOD: 625/8270 LAB SOPS:F.D.05.01\& F.D.03.02 . - Page - 2

Name Field: $12-13-1-$ TK OIL Operator: KRAIG Misc Field: $9209048-18 \mathrm{~A}$ Instrument: BNA1 Datafile: >SDI16 Idfile: ID_BCI Quantfile: ^SDI16

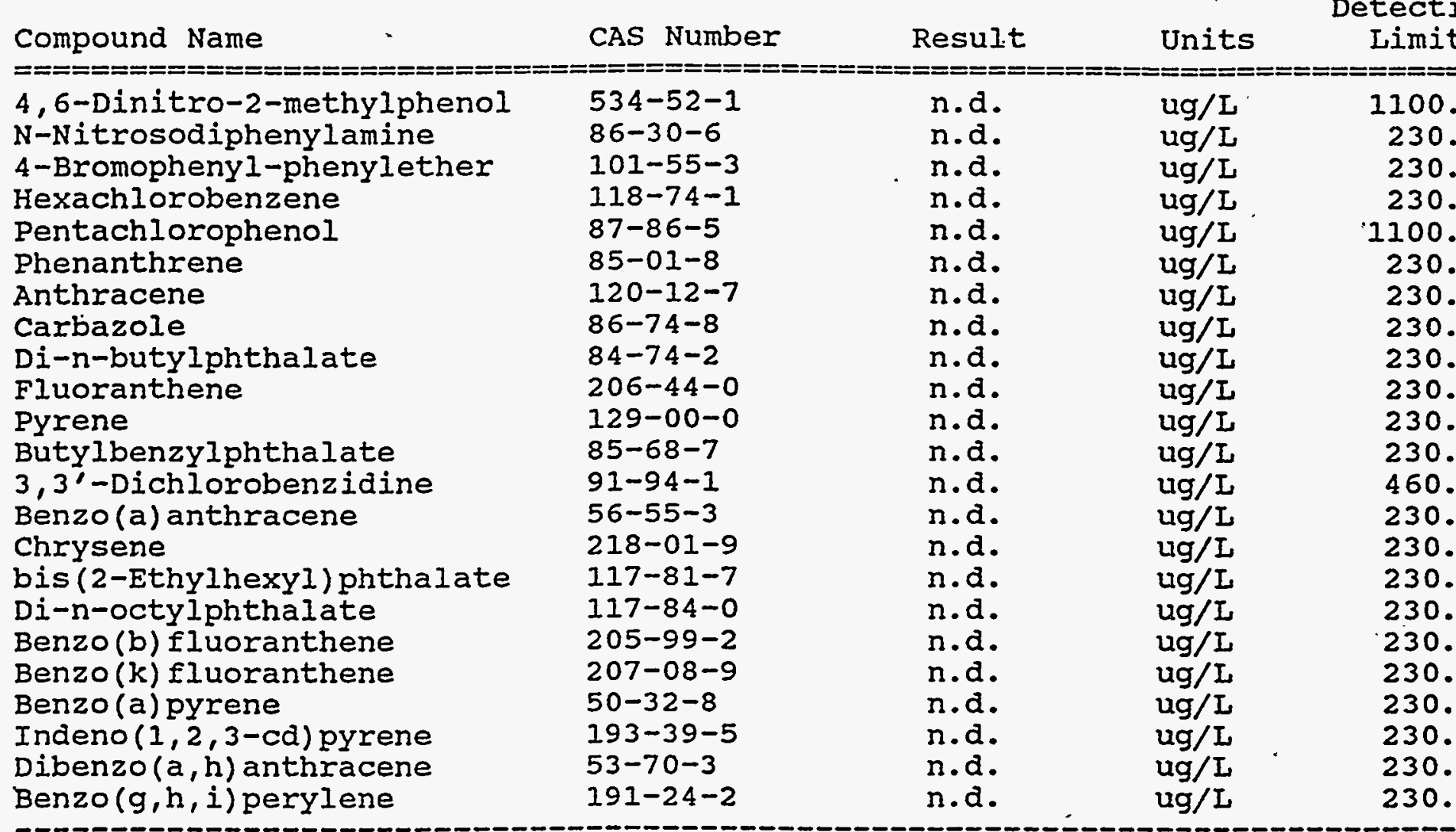

n.d. - not detected

Approved by: 
REECO ANALYTICAL CHEMISTRY “LAB'S SEMIVOLATIEE ORGANICFE'TEST RESULTS EPA METHOD:625/8270 LAB SOPS:F.D.05.01\& F.D.03.02

- Page - 1

Name Field: 12-13-1-TK WATER

Operator: KRAIG

Misc Field: $9209048-18 B$ 2ND RUN

Instrument: BNA2 Datafile: >SD276

Idfile: ID_BC2 Quantfile: ^SD276

Injection time: $19: 51$ on 10-07-92

Continuing calibration time : $16: 44$

User dilfac:45.9 Quant dilfac:

Quant Time: $09: 13$ on 10-08-92

on 10-07-92 using the file: ^SC243::D5

1. Multi-calibration: 15:20 on 06-24-92

Compound Name

CAS Number

Result

Detectior

Phenol

bis (2-chloroethyl) ether

2-Chlorophenol

1,3-Dichlorobenzene

1,4-Dichlorobenzene

Benzyl alcohol

1,2-Dichlorobenzene

2-Methylphenol

$2,2^{\prime}$-oxybis (1-Chloropropane)

bis (2-Chloroisopropyl) ether

4-Methylphenol

N-Nitroso-di-n-propylamine

Hexachloroethane

Nitrobenzene

Isophorone

2-Nitrophenol

2,4-Dimethylphenol

Benzoic acid

bis (2-Chloroethoxy) methane

2,4-Dichlorophenol

1,2,4-Trichlorobenzene

Naphthalene

4-Chloroaniline

Hexachlorobutadiene

4-Chloro-3-methylphenol

2-Methylnaphthalene

Hexachlorocyclopentadiene

2, 4, 6-Trichlorophenol

$2,4,5-T r i c h l o r o p h e n o l$

2-Chloronaphthalene

2-Nitroaniline

Dimethylphthalate

Acenaphthylene

2,6-Dinitrotoluene

3-Nitroaniline

Acenaphthene

2,4-Dinitrophenol

4-Nitrophenol

Dibenzofuran

2,4-Dinitrotoluene

Diethylphthalate

4-Chlorophenyl-phenylether

Fluorene

4-Nitroaniline

$\begin{array}{ll}108-95-2 & \text { n.d. } \\ 111-44-4 & \text { n.d. } \\ 95-57-8 & \text { n.d. } \\ 541-73-1 & \text { n.d. } \\ 106-46-7 & \text { n.d. } \\ 100-51-6 & \text { n.d. } \\ 95-50-1 & \text { n.d. } \\ 95-48-7 & \text { n.d. } \\ 108-60-1 & \text { n.d. } \\ 39638-32-9 & \text { n.d. } \\ 106-44-5 & \text { n.d. } \\ 621-64-7 & \text { n.d. } \\ 67-72-1 & \text { n.d. } \\ 98-95-3 & \text { n.d. } \\ 78-59-1 & \text { n.d. } \\ 88-75-5 & \text { n.d. } \\ 105-67-9 & \text { n.d. } \\ 65-85-0 & \text { n.d. } \\ 111-91-1 & \text { n.d. } \\ 120-83-2 & \text { n.d. } \\ 120-82-1 & \text { n.d. } \\ 91-20-3 & \text { n.d. } \\ 106-47-8 & \text { n.d. } \\ 87-68-3 & \text { n.d. } \\ 59-50-7 & \text { n.d. } \\ 91-57-6 & \text { n.d. } \\ 77-47-4 & \text { n.d. } \\ 88-06-2 & \text { n.d. } \\ 95-95-4 & \text { n.d. } \\ 91-58-7 & \text { n.d. } \\ 88-74-4 & \text { n.d. } \\ 131-11-3 & \text { n.d. } \\ 208-96-8 & \text { n.d. } \\ 606-20-2 & \text { n.d. } \\ 99-09-2 & \text { n.d. } \\ 83-32-9 & \text { n.d. } \\ 51-28-5 & \text { n.d. } \\ 100-02-7 & \text { n.d. } \\ 132-64-9 & \text { n.d. } \\ 121-14-2 & \text { n.d. } \\ 84-66-2 & \text { n.d. } \\ 7005-72-3 & \text { n.d. } \\ 86-73-7 & \text { n.d. } \\ 100-01-6 & \text { n.d. }\end{array}$

Units

Iimit

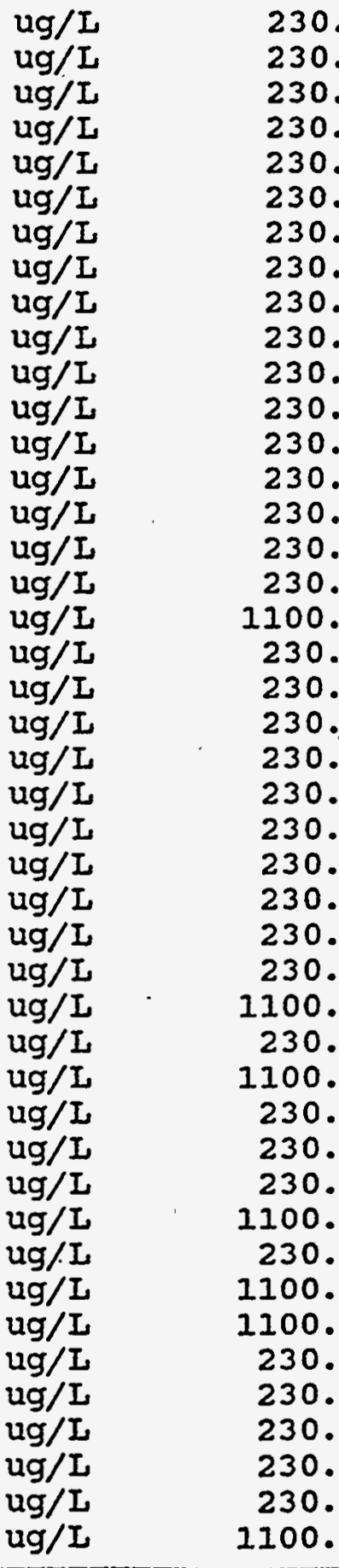

230.

230.

230.

230.

230.

230.

230 .

230 .

230 .

230 .

230 .

230.

230.

230 .

230 .

230 .

30.

330.

230.

230 .

230 .

100.

230 .

100.

230.

100.

230.

1100 .

100.

230.

230 .

230.

230.

1100. 
REECO ANALYTICAL CHEMISTRY LAB'S SEMIVOLATILE ORGANICS TEST RESUITS

EPA METHOD:625/8270 LAB SOPS:F.D.05.01 \& F.D.03.02

- Page - 2

Name Field: 12-13-1-TK WATER

Misc Field: 9209048-18B 2ND RUN

Instrument: BNA2 Datafile: >SD276 Iafile: ID_BC2 Quantfile: ^SD276

Operator: KRAIG

\begin{tabular}{|c|c|c|c|c|}
\hline 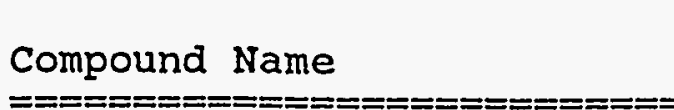 & $\begin{array}{l}\text { CAS Number } \\
==========\end{array}$ & $\begin{array}{l}\text { Result } \\
========\end{array}$ & Units & $\begin{array}{l}\text { etecti } \\
\text { Iimit }\end{array}$ \\
\hline $\begin{array}{l}\text { 6-Dinitro-2-methylphenol } \\
\text {-Nitrosodiphenylamine } \\
\text { - Bromophenyl-phenylether } \\
\text { exachlorobenzene } \\
\text { entachlorophenol } \\
\text { lenanthrene } \\
\text { ithracene } \\
\text { arbazole } \\
\text {-n-butylphthalate } \\
\text { luoranthene } \\
\text { rene } \\
\text { dtylbenzylphthalate } \\
\text { 3'-Dichlorobenzidine } \\
\text { enzo(a) anthracene } \\
\text { irysene } \\
\text { is(2-Ethylhexyl) phthalate } \\
\text {-n-octylphthalate } \\
\text { enzo(b) fluoranthene } \\
\text { enzo(k) fluoranthene } \\
\text { enzo(a) pyrene } \\
\text { deno(l,2, 3-cd) pyrene } \\
\text { benzo(a, h) anthracene } \\
\text { enzo(g, h, i) perylene }\end{array}$ & $\begin{array}{l}534-52-1 \\
86-30-6 \\
101-55-3 \\
118-74-1 \\
87-86-5 \\
85-01-8 \\
120-12-7 \\
86-74-8 \\
84-74-2 \\
206-44-0 \\
129-00-0 \\
85-68-7 \\
91-94-1 \\
56-55-3 \\
218-01-9 \\
117-81-7 \\
117-84-0 \\
205-99-2 \\
207-08-9 \\
50-32-8 \\
193-39-5 \\
53-70-3 \\
191-24-2\end{array}$ & 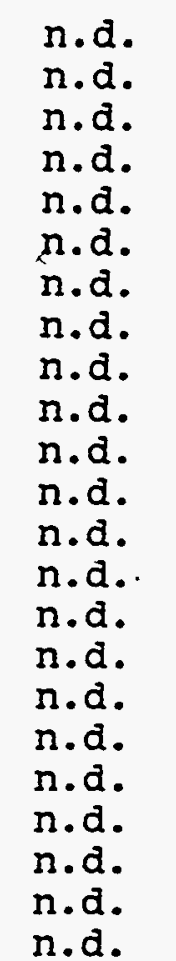 & $\begin{array}{l}\mathrm{ug} / I \\
\mathrm{ug} / I \\
u g / I \\
u g / I \\
u g / I \\
u g / I \\
u g / I \\
u g / I \\
u g / I \\
u g / I \\
u g / I \\
u g / I \\
u g / I \\
u g / I \\
u g / I \\
u g / I \\
u g / I \\
u g / I \\
u g / I \\
u g / I \\
u g / I \\
u g / I \\
u g / I\end{array}$ & 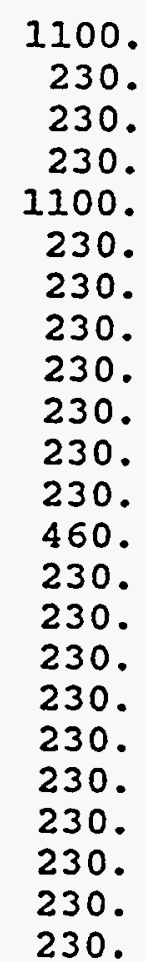 \\
\hline
\end{tabular}

n.d. - not detected

Approved by: 
REECO ANALYTICAL CHEMISTRY LAB'S SEMIVOLATILE ORGANICS“"TEST RESULTS

EPA METHOD: 625/8270 LAB SOPS:F.D.05.01 \& F.D.03.02

- Page - 1

Name Field: 12-13-1-TK MS OIL

Operator: KRAIG

Misc Field: $9209048-27 \mathrm{~A}$

Instrument: BNA1 Datafile: $>S D 117$

Idfile: ID_BCI Quantfile: ^SD117

Injection time: $16: 25$ on 10-07-92

Continuing calibration time : $12: 04$

User dilfac:42.2 Quant dilfac:

Quant Time: $17: 16$ on 10-07-92

on 10-07-92 using the file: ^sC154::D4

1. Multi-calibration: $10: 28$ on $09-22-92$

Compound Name

Phenol

bis (2-Chloroethyl) ether

2-Chlorophenol

1,3-Dichlorobenzene

1,4-Dichlorobenzene

Benzyl alcohol

1,2-Dichlorobenzene

2-Methylphenol

$2,2^{\prime}$-oxybis ( 1 -Chloropropane)

bis (2-chloroisopropyl) ether

4-Methylphenol

N-Nitroso-di-n-propylamine

Hexachloroethane

Nitrobenzene

Isophorone

2-Nitrophenol

2,4-Dimethylphenol

Benzoic acid

bis (2-Chloroethoxy) methane

2,4-Dichlorophenol

$1,2,4$-Trichlorobenzene

Naphthalene

4-Chloroaniline

Hexachlorobutadiene

4-ChIoro-3-methylphenol

2-Methylnaphthalene

Hexachlorocyclopentadiene

$2,4,6$-Trichlorophenol

$2,4,5$-Trichlorophenol

2-Chloronaphthalene

2-Nitroaniline

Dimethylphthalate

Acenaphthylene

2, 6-Dinitrotoluene

3-Nitroaniline

Acenaphthene

2,4-Dinitrophenol

4-Nitrophenol

Dibenzofuran

2,4-Dinitrotoluene

Diethylphthalate

4-Chlorophenyl-phenylether

Fluorene

4-Nitroaniline
CAS Number

$108-95-2$

$111-44-4$

95-57-8

541-73-1

106-46-7

$100-51-6$

95-50-1

$95-48-7$

108-60-1

39638-32-9

106-44-5

621-64-7

$67-72-1$

98-95-3

$78-59-1$

$88-75-5$

105-67-9

65-85-0

111-91-1

120-83-2

120-82-1

91-20-3

106-47-8

$87-68-3$

59-50-7

$91-57-6$

$77-47-4$

88-06-2

95-95-4

91-58-7

$88-74-4$

$131-11-3$

208-96-8

606-20-2

99-09-2

$83-32-9$

$51-28-5$

100-02-7

132-64-9

121-14-2

$84-66-2$

$7005-72-3$

$86-73-7$

100-01-6
Result

840 .

n.d.

830 .

410 .

390 .

n.d.

n.d.

n.d.

n.d.

n.d.

n.d.

500 .

n.d.

n.d.

58.

n.d.

n.d.

n.d.

n.d.

n.d.

370 .

290.

110.

n.d.

820 .

1100 .

n.d.

n.d.

n.d.

n.d.

n.d.

n.d.

n.d.

n.d.

n.d.

450 .

n.d.

n.d.

82.

320 .

n.d.

n.d.

150.

n.d.

Detectior. Limit

210.

210 .

210.

210.

210.

210.

210.

210.

210.

210.

210.

210.

210.

210.

210.

210.

210.

1100.

210.

210.

210.

210.

210.

210.

210.

210.

210.

210.

1100.

210.

1100.

210 .

210.

210.

1100 .

210.

1100.

1100 .

210.

210.

210 .

210.

210 .

1100. 
REECO ANALYTICAL CHEMISTRY LAB'S SEMIVOLATILE ORGANICS TEST RESULTS

EPA METHOD: 625/8270 LAB SOPS:F.D.05.01 \& F.D.03.02

- Page - 2

Name Field: 12-13-1-TK MS OIL Operator: KRAIG

Misc Field: $9209048-27 \mathrm{~A}$

Instrument: BNA1 Datafile: >SD117

Idfile: ID_BCl Quantfile:

$\wedge$ SD117

Compound Name

CAS Number

Result

Detectio:

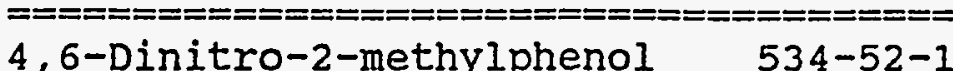

$\mathrm{N}-\mathrm{Nitrosodiphenylamine}$

$86-30-6$

4-Bromopheny 1-phenylether

$101-55-3$

Hexachlorobenzene

Pentachlorophenol

Phenanthrene

Anthracene

Carbazole

Di-n-butylphthalate

Fluoranthene

Pyrene

Butylbenzylphthalate

$3,3^{\prime}$-Dichlorobenzidine

$118-74-1$

$87-86-5$

$85-01-8$

$120-12-7$

$86-74-8$

84-74-2

$206-44-0$

$129-00-0^{\circ}$

85-68-7

$91-94-1$

Benzo (a) anthracene

$56-55-3$

Chrysene

218-01-9

bis (2-Ethylhexyl) phthalate

$117-81-7$

Di-n-octylphthalate

$117-84-0$

Benzo (b) fluoranthene

205-99-2

Benzo (k) fluoranthene

207-08-9

Benzo (a) pyrene

$50-32-8$

Indeno $(1,2,3-c d)$ pyrene

193-39-5

Dibenzo $(a, h)$ anthracene

53-70-3

Benzo $(g, h, i)$ perylene

191-24-2

n.d.

n.d.

n.d.

n.d.

400 .

230 .

230 .

n.d.

n.d.

n.d.

230 .

n.d.

n.d.

n.d.

n.d.

n.d.

n.d.

n.d.

n.d.

n.d.

n.d.

n.d.

n.d.

Units

Limit

- Benzo $(g, h$, d perylene

n.d. - not detected

Approved by: 
REECO ANALYTICAL CHEMISTRY LAB'S SEMIVOLATILE ORGANICS TEST RESULTS EPA METHOD: 625/8270 IAB SOPS:F.D.05.01\& F.D.03.02

- Page - 1

Name Field: 12-13-1-TK MSD OIL

Operator: KRAIG

Misc Field: $9209048-28 \mathrm{~A}$

Instrument: BNAI Datafile: >SD118 Idfile: ID_BCI Quantfile: ^SDI18

Injection time: $17: 27$ on 10-07-92

Continuing calibration time : $12: 04$ on 10-07-92 using the file: ^SC154::D4 User dilfac:50 Quant dilfac:

1. Multi-calibration: $10: 28$ on $09-22-92$

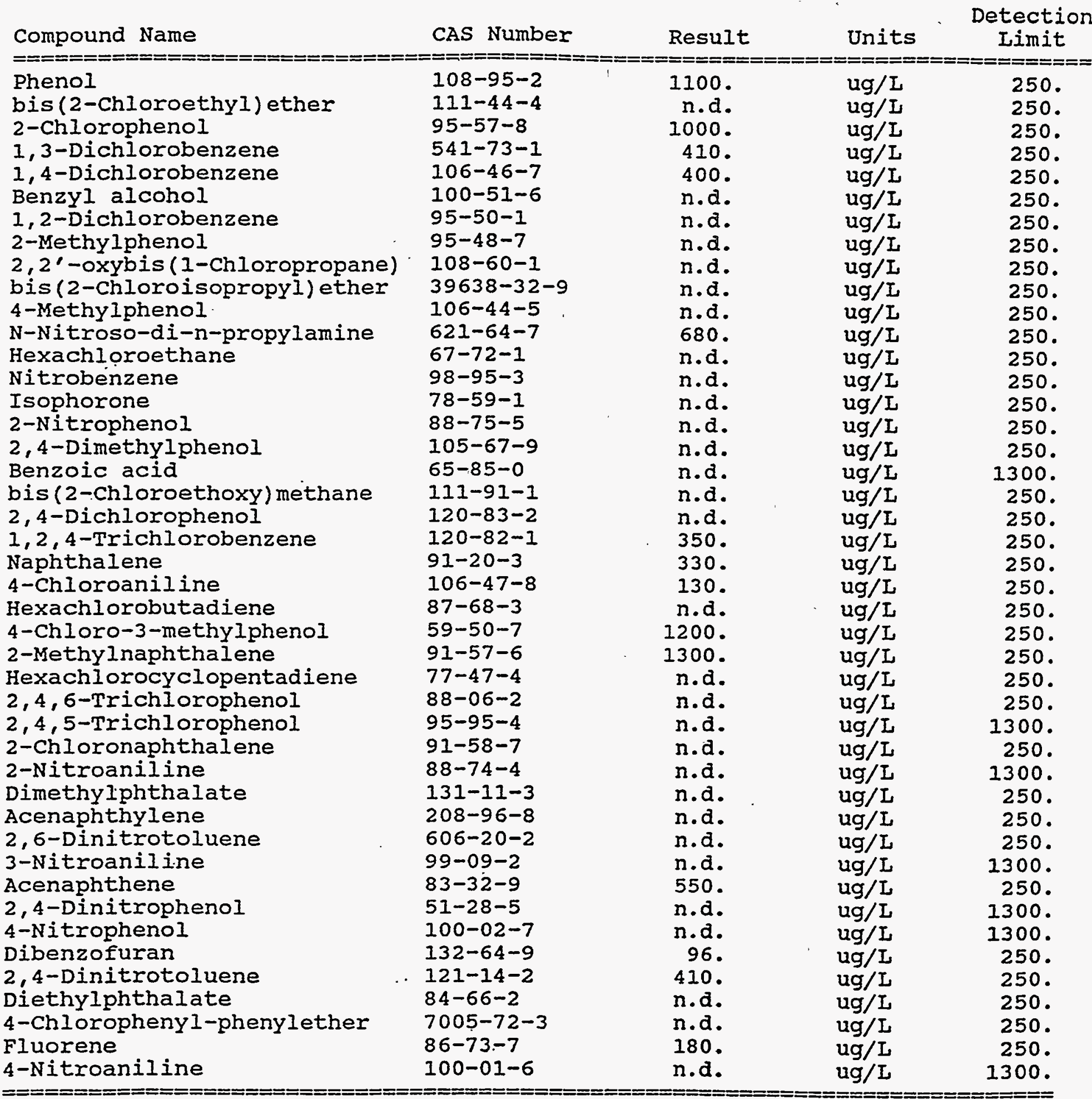


REECO ANALYTICAI CHEMISTRY LAB'S SEMIVOLATILE ORGANICS TEST RESULTS

EPA METHOD: 625/8270 LAB SOPS:F.D.05.01 \& F.D.03.02

- Page - 2

Name Field: 12-13-1-TK MSD OIL

Operator: KRAIG

MisC Field: $9209048-28 \mathrm{~A}$

Instrument: BNAI Datafile: >SD1I8

Idfile: ID_BCl Quantfile: ^SD118

Compound Name

- CAS Number

Result

Detectic

$=====================$
$4,6-$ Dinitro-2-methylphenol

$\mathrm{N}-\mathrm{Nitrosodiphenylamine}$

4-Bromophenyl-phenylether

$534-52-1$

$86-30-6$

$101-55-3$

Hexachlorobenzene

$118-74-1$

Pentachlorophenol

Phenanthrene

Anthracene

Carbazole

Di-n-butylphthalate

$87-86-5$

Fluoranthene

85-01-8

$120-12-7$

$86-74-8$

$84-74-2$

Pyrene

206-44-0

129-00-0

n.d.

170 .

n.d.

n.d.

450 .

230 .

240 .

n.a.

n.d.

n.d.

85-68-7

130 .

Butylbenzylphthalate

$91-94-1$

Benzo (a) anthracene

$56-55-3$

Chrysene

218-01-9

bis (2-Ethylhexyl) phthalate

$117-81-7$

Di-n-octylphthalate

117-84-0

205-99-2

207-08-9

n.d.

n.d.

n.d.

n.d.

n.d.

n.d.

n.d.

Benzo(k) fluoranthene

$50-32-8$

Benzo (a) pyrene

n.d.

n.d.

$193-39-5$

n.d.

$53-70-3$

Dibenzo $(a, h)$ anthracene

191-24-2

n.d.

n.d.

Units Iimit

Benzo $(g, h, i)$ perylene

ug/L

$\mathrm{ug} / \mathrm{I}$

1300 .

$\mathrm{ug} / \mathrm{L}$

250 .

250 .

250 .

13.00 .

$\mathrm{ug} / \mathrm{I}$

250 .

ug/L

250 .

$\mathrm{ug} / \mathrm{I}$

250 .

$\mathrm{ug} / \mathrm{I}$

250 .

250 .

250 .

250 .

500 .

250.

250 .

250 .

250 .

250 .

250 .

250 .

250 .

250 .

250.

n.d. - not detected

Approved by: 
REECO ANALYTICAL CHEMISTRY LAB'S SEMIVOLATILE ORGANICS TEST RESULTS

EPA METHOD:625/8270 LAB SOPS:F.D.05.01\&F.D.03.02 - Page - 1

Name Field: SBLK 0921

Operator: KRrig

Misc Field: $9209048-26 \mathrm{~A}$

Instrument: BNA2 Datafile: >SD269 Idfile: ID_BC2 Quantfile: ^SD269

Injection time: $09: 28$ on 10-07-92

Continuing calibration time : 05:08 on 10-07-92 using the file: ^SCS22::QT

User dilfac:2 Quant dilfac: 1. Multi-calibration: 15:20 on 06-24-92

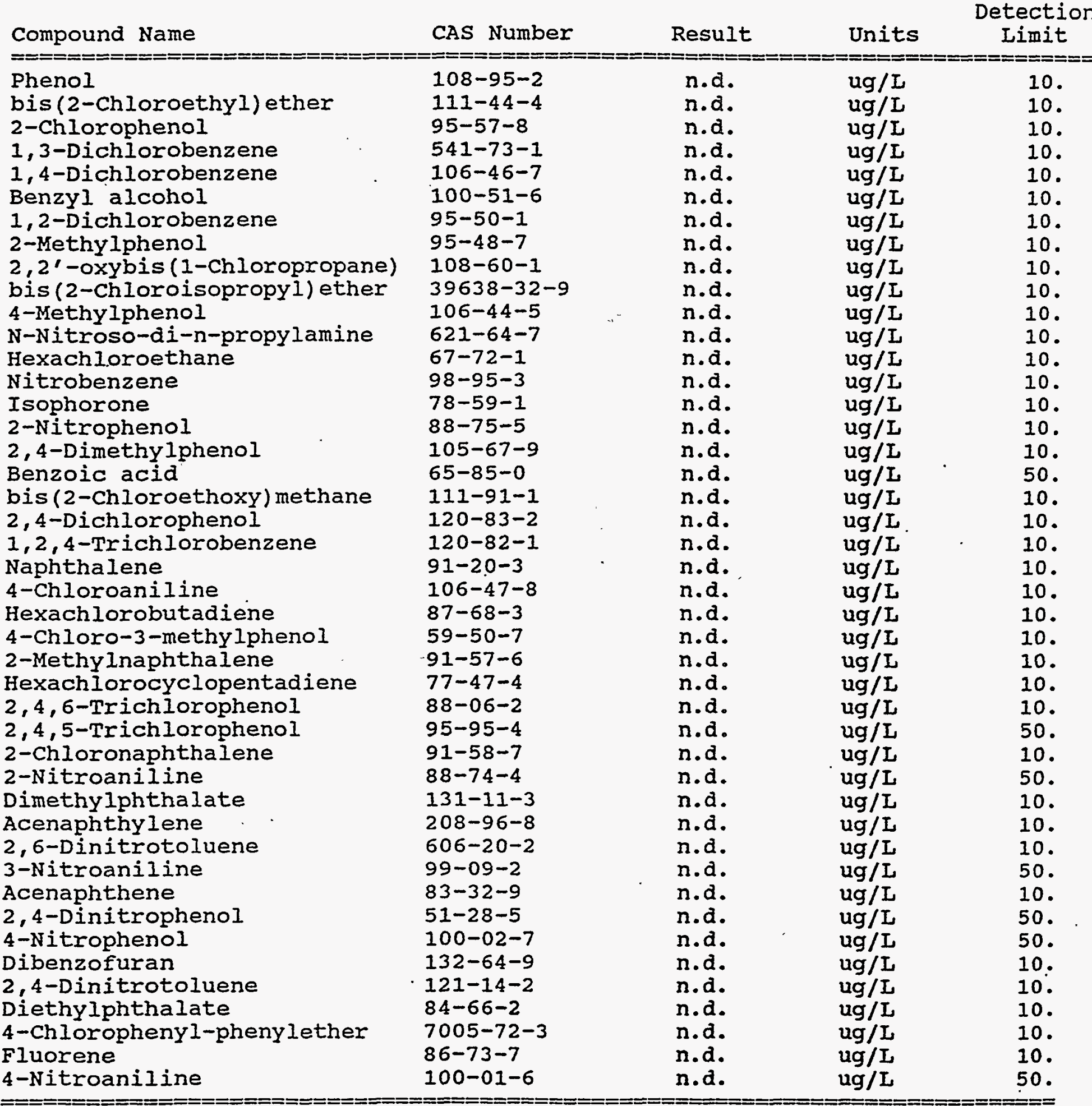


REECO ANALYTICAL CHEMISTRY LAB'S SEMIVOLATILE ORGANICS TEST RESULTS

EPA METHOD:625/8270 LAB SOPS:F.D.05.01\& F.D.03.02

- Page - 2

Name Fieid: SBLK 0921

Misc Field: $9209048-26 \mathrm{~A}$

Instrument: BNA2 Datafile: >SD269 Idfile: ID_BC2 Quantfile: ^SD269

Operator: KRAIG

Compound Name

CAS Number

Result

Detectior

4,6-Dinitro-2-methylphenol $\quad 534-52-1$

N-Nitrosodiphenylamine

4-Bromophenyl-phenylether

Hexachlorobenzene

Pentachlorophenol

Phenanthrene

Anthracene

Carbazole

Di-n-butylphthalate

Fluoranthene

Pyrene

Butylbenzylphthalate

$3,3^{\prime}-$ Dichlorobenzidine

Benzo (a) anthracene

$86-30-6$

101-55-3

$118-74-1$

$87-86-5$

$85-01-8$

$120-12-7$

$86-74-8$

$84-74-2$

206-44-0

129-00-0

$85-68-7$

91-94-1

Chrysene

56-55-3

bis (2-Ethylhexyl) phthalate

218-01-9

$117-81-7$

n.d.

n.d.

n.d.

n.d.

n.d.

n.d.

n.d.

n.d.

n.d.

n.d.

n.d.

n.d.

n.d.

n.d.

n.d.

$117-84-0$

n.d.

205-99-2

Benzo (b) fluoranthene

Benzo(k) fluoranthene

Benzo (a) pyrene

Indeno $(1,2,3-c d)$ pyrene

Dibenzo $(a, h)$ anthracene

$\operatorname{Benzo}(g, h, i)$ perylene

207-08-9

n.d.

n.d.

n.d.

n.d.

193-39-5

n.d.

$53-70-3$

n.d.

191-24-2

n.d.

Units Limit

$\begin{array}{ll}\mathrm{ug} / \mathrm{L} & 50 . \\ \mathrm{ug} / L & 10 . \\ \mathrm{ug} / \mathrm{L} & 10 . \\ \mathrm{ug} / \mathrm{L} & 10 . \\ \mathrm{ug} / \mathrm{L} & 5.0 \\ \mathrm{ug} / \mathrm{L} & 10 . \\ \mathrm{ug} / \mathrm{L} & 10 . \\ \mathrm{ug} / \mathrm{L} & 10 . \\ \mathrm{ug} / \mathrm{L} & 10 . \\ \mathrm{ug} / \mathrm{I} & 10 . \\ \mathrm{ug} / \mathrm{L} & 10 . \\ \mathrm{ug} / \mathrm{L} & 10 . \\ \mathrm{ug} / \mathrm{L} & 20 . \\ \mathrm{ug} / \mathrm{L} & 10 . \\ \mathrm{ug} / \mathrm{L} & 10 . \\ \mathrm{ug} / \mathrm{L} & 10 . \\ \mathrm{ug} / \mathrm{L} & 10 . \\ \mathrm{ug} / \mathrm{L} & 10 . \\ \mathrm{ug} / \mathrm{L} & 10 . \\ \mathrm{ug} / \mathrm{L} & 10 . \\ \mathrm{ug} / \mathrm{L} & 10 . \\ \mathrm{ug} / \mathrm{L} & 10 . \\ \mathrm{ug} / \mathrm{L} & 10 .\end{array}$

n.d. - not detected

Approved by: 
$\rightarrow$

SUPPORTING DATA 
(1) $\quad 2^{\circ} \mathrm{C}$

Reynolds Electrical \& Engineering Co., Inc.

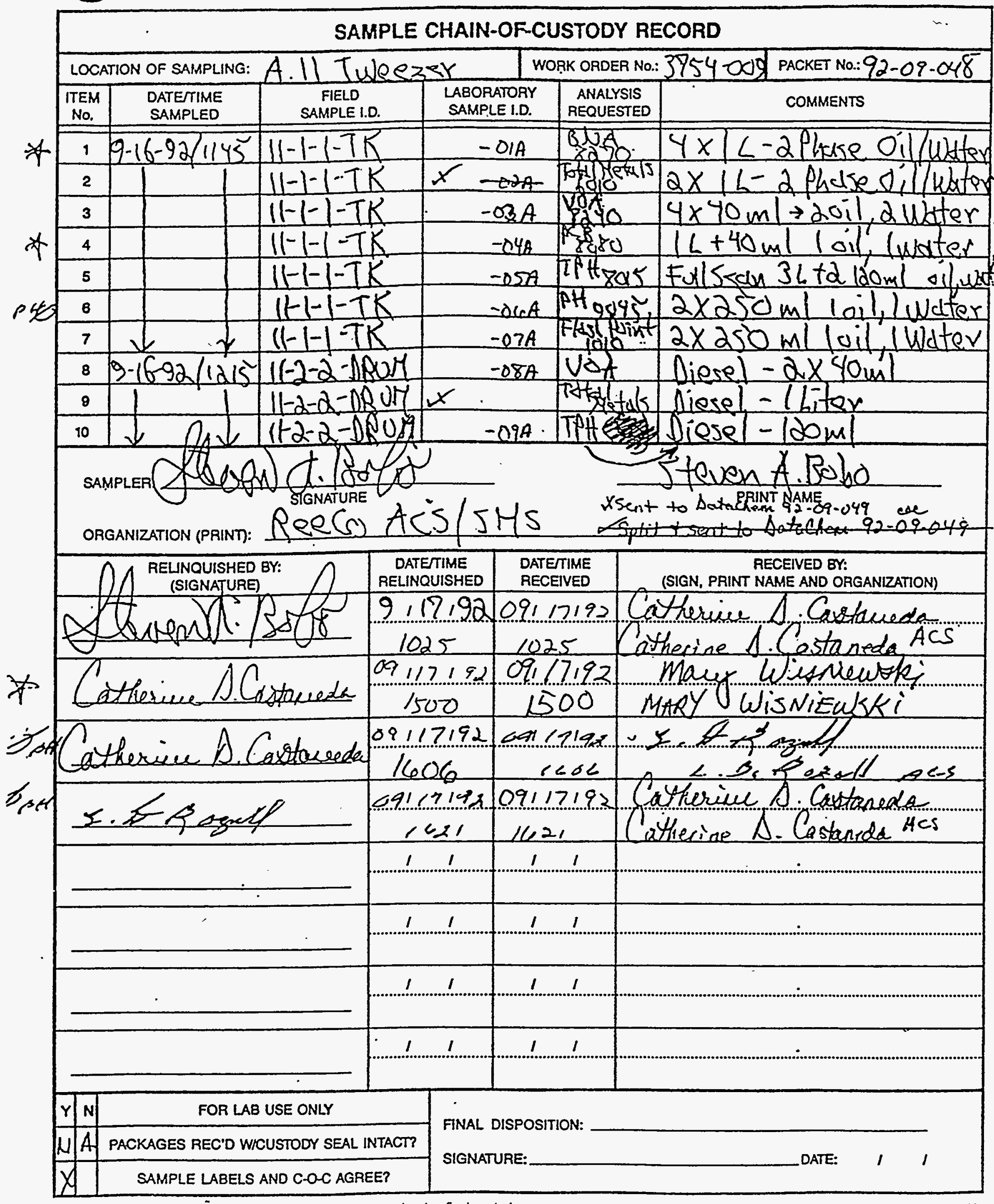




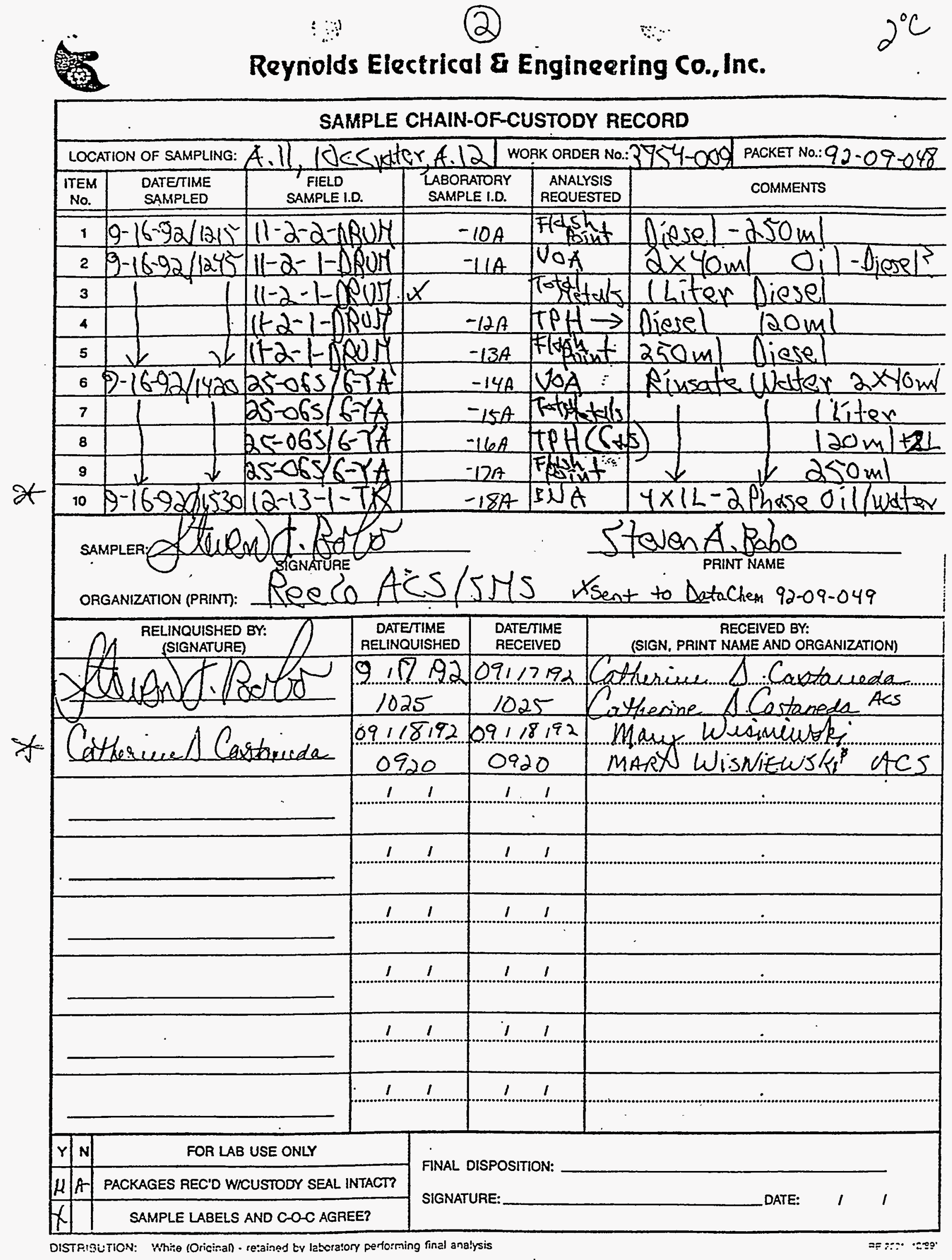




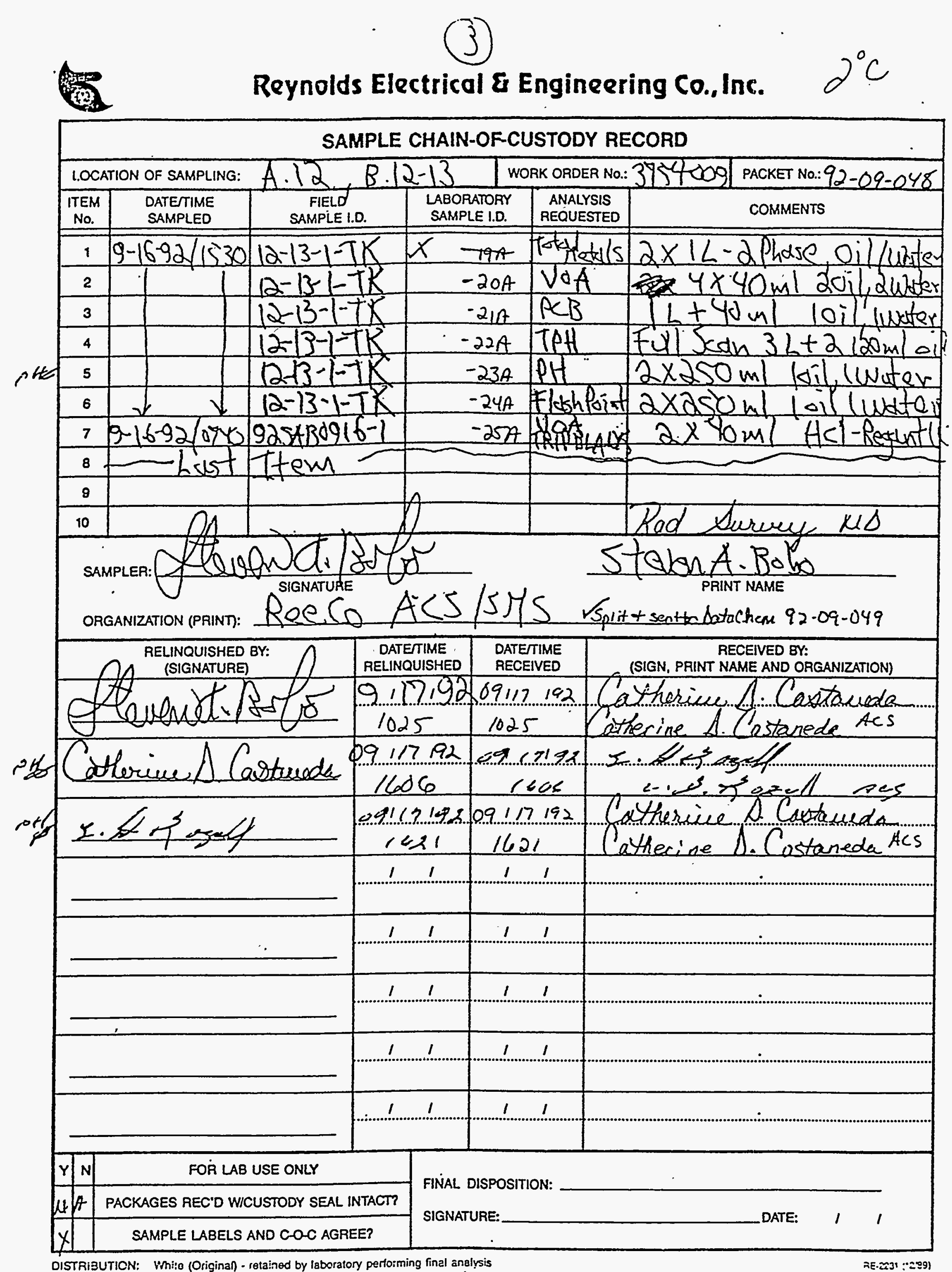


REYNUL. I ELECTRICAL AND ENGINEERI، . COMPANY

ANALYTICAL SERVICES DEPARTMENT

SAMPLE ANALYSIS REQUEST SHEET

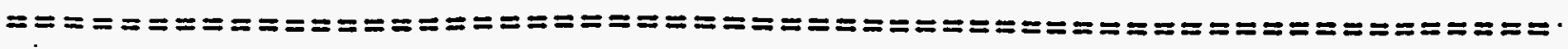

w.o.x: $3754-009$

DATE SUBMITTED: $9 / 15 / 92$

DATE REQUired: $9 / 1 \mathrm{C} / 92$

REQUESTER: DVD. MADSEN

PHONE: $5-7211$ DEPT: ECO

Mrs: $7 / 1$

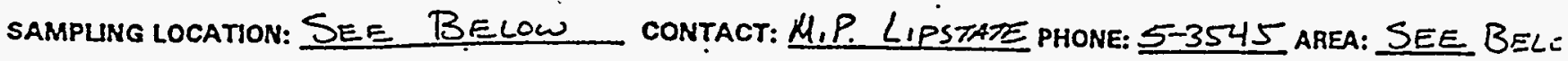

RÁD ANALYSIS REQUIRED:

CHEMICAL ANALYSIS REQUIRED: YES No PRIORITY CODE: Rush

TYPE REPORT REQUIRED:

X 1. TCL results only.

2. TCL and $Q C$ results.

3. TCL and THC results.

- 4. TCL, TIC, and $Q C$ results.

5. CLP.TYPE Report.

SPECLA INSTRUCTIONS: only one MS +MSD to be be analyzed for batch,

- sample Numbers + analyses regressed on tack - Note Data chem For samples submitted for Foal Metals Analysis (only need TCLP metals to be reported)

Date report Is ReQuired: $10 / 1 / 9 \mathrm{Z}$ REQUestor signature:

DESCRIPTION OF WASTE:

1. NUMBER OF SAMPLES:

2. TYPE(S) QF WASTE CONTANERS: (3) lindergroand storage tents and (1) 55 gallon drum

REQUestor signature:

SAMPLER: $S$. Rob

ALD PACKET : $92-09-049$

DATE COURTED: 0$)-(b-0)$

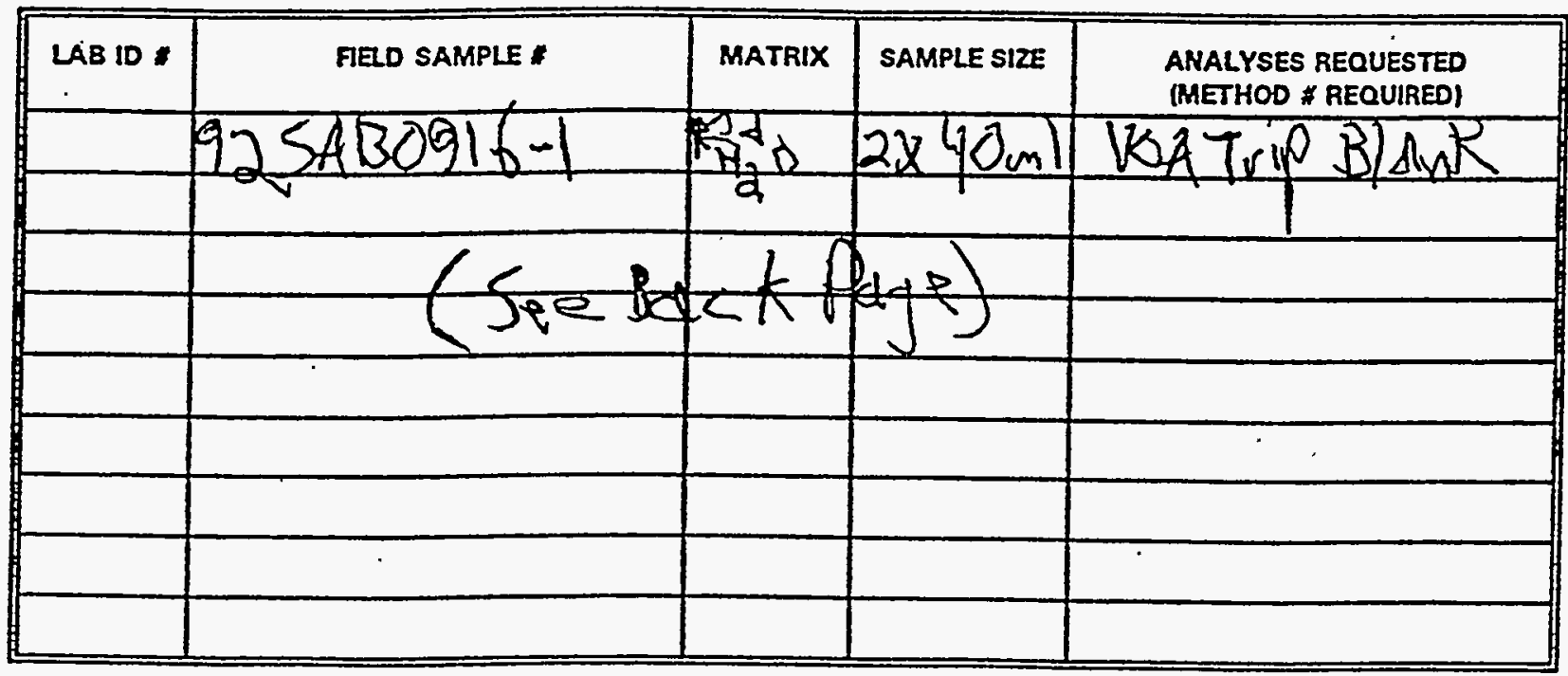

COMMENTS: 


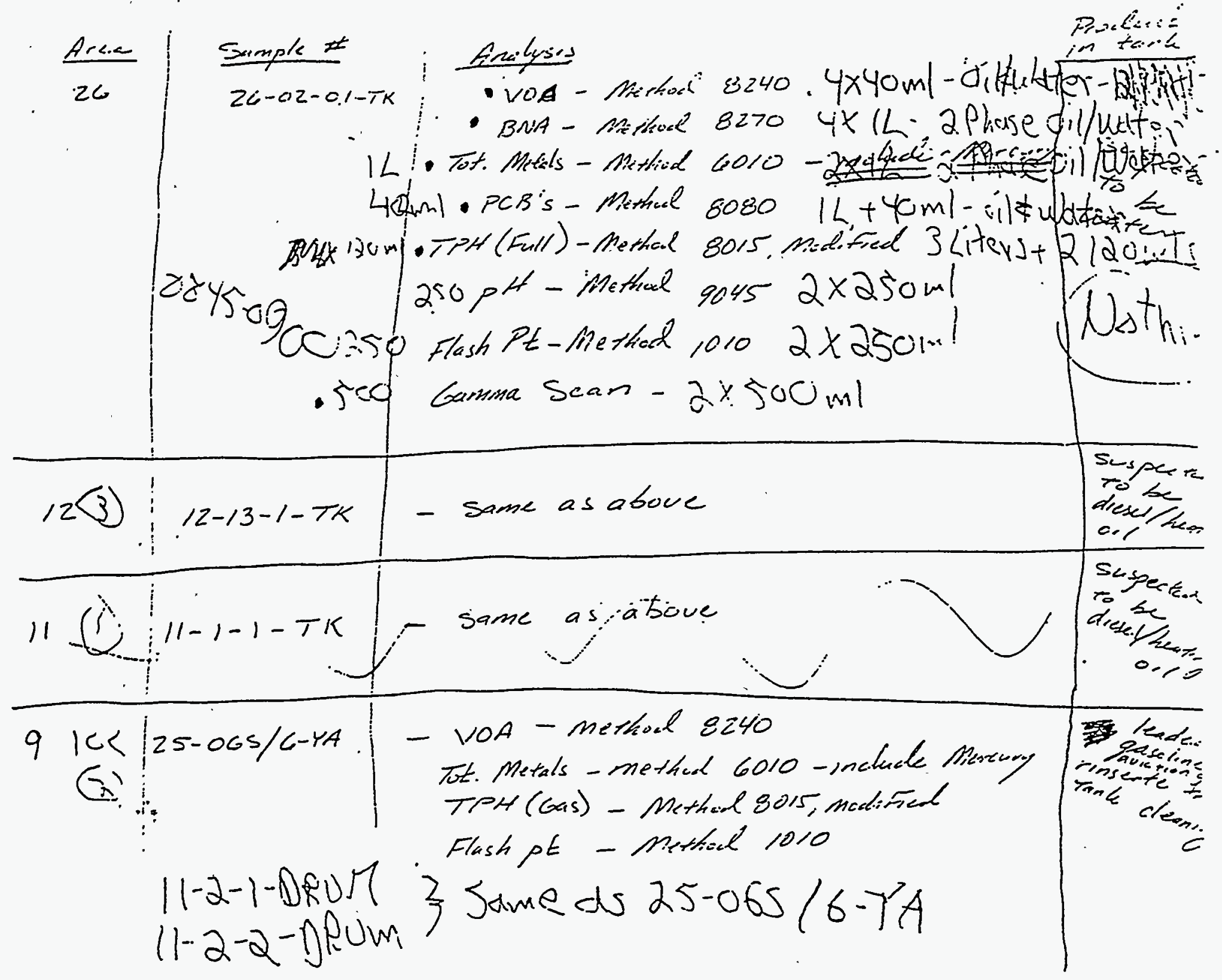


LE

11

sk. 2.

m Page No.

Sample hocations: Ared 26 Bldg. 2105; Avea 11 Tweeze Times. Facility; $10<$ Crater; Alea 12, Bldd $12-13$

Tests: $01,115,1215,1245,1420$ and 530 hours

a Diesel) A Total Tefals, Pctss, TPH (Full, 6ds

For: Dave Massen, EO zokelipstate, NTS Geneve

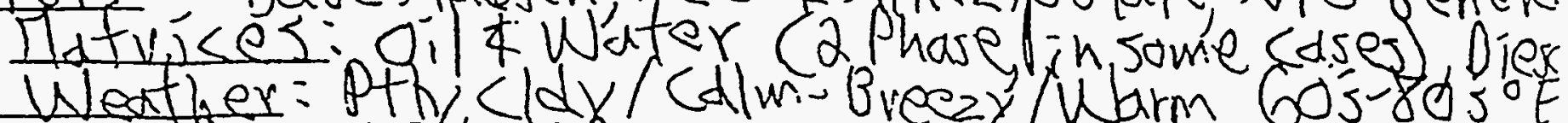

$1,0 .+375 / 4.004$

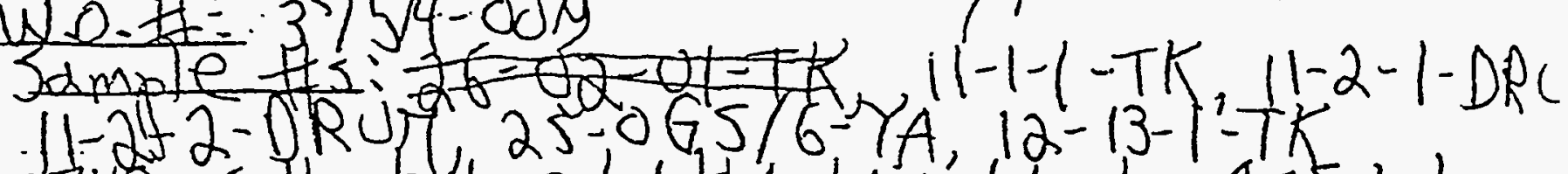

0740 - sullect! jet Ud A trip blanks, ac5 lasb Preserve with 3 arops HitCl $\rightarrow$ Yoml Readnt $H_{2} \mathrm{O}$ o 840-Arrive at Area 26 Blide. 105 . A fremana. and a laboer remoses tae top of the ust. at the SE Corner at the buitalind The tant. was campleteryempty so no sande lewess taken Tom wilcelt of

1000 - accompdny me ar the doly het site at oge Avruelat Aved is Tueezer facility, with Eco. with great dif enerd a an wikek

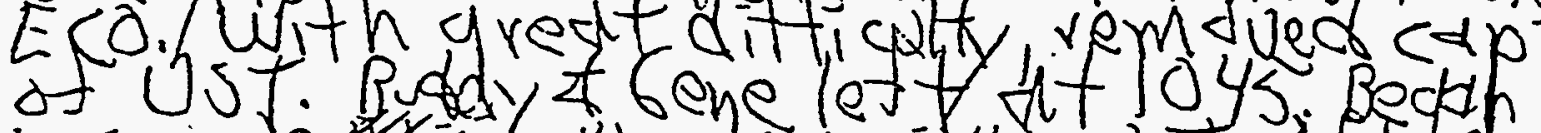
to sample "heating oil" inUST. Af ed 11-1-1-TK teping the soliwdse to, d. Woden dip stick

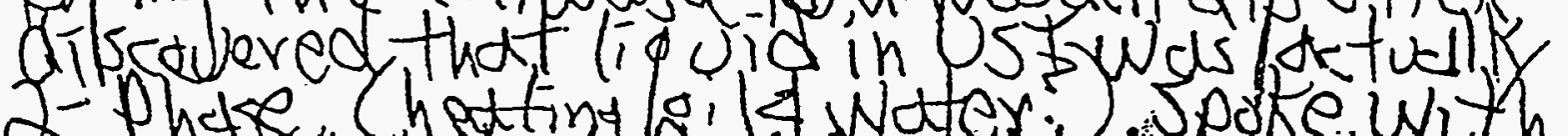

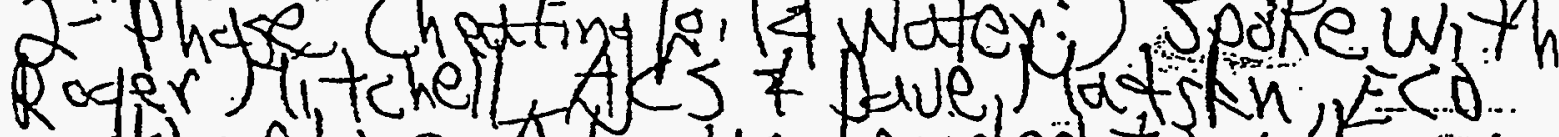

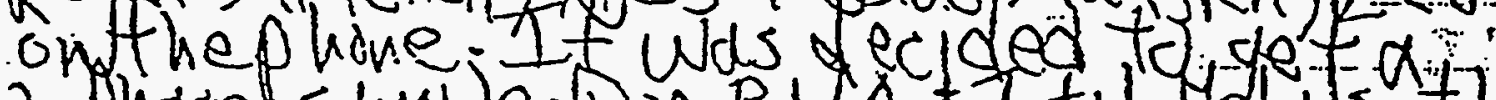
2 phaser sumpe far BNA \& Tofal Metals, then try to sepdrate the oil from the water $*$ geft wicel ds many samples toy the rest of thet Shis was done; and wasluery time cousuminat

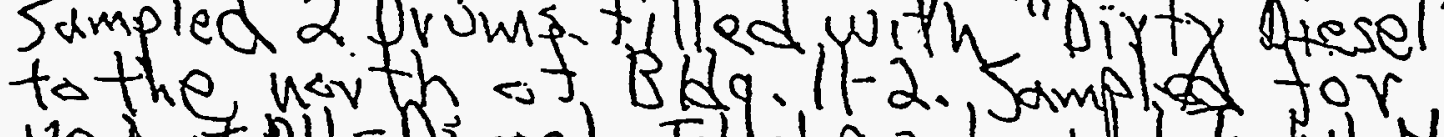

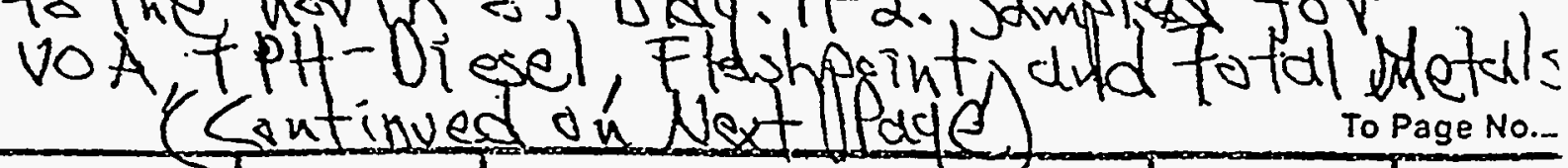

i.essed \& Understood by me,

Date

Invented by

Date 


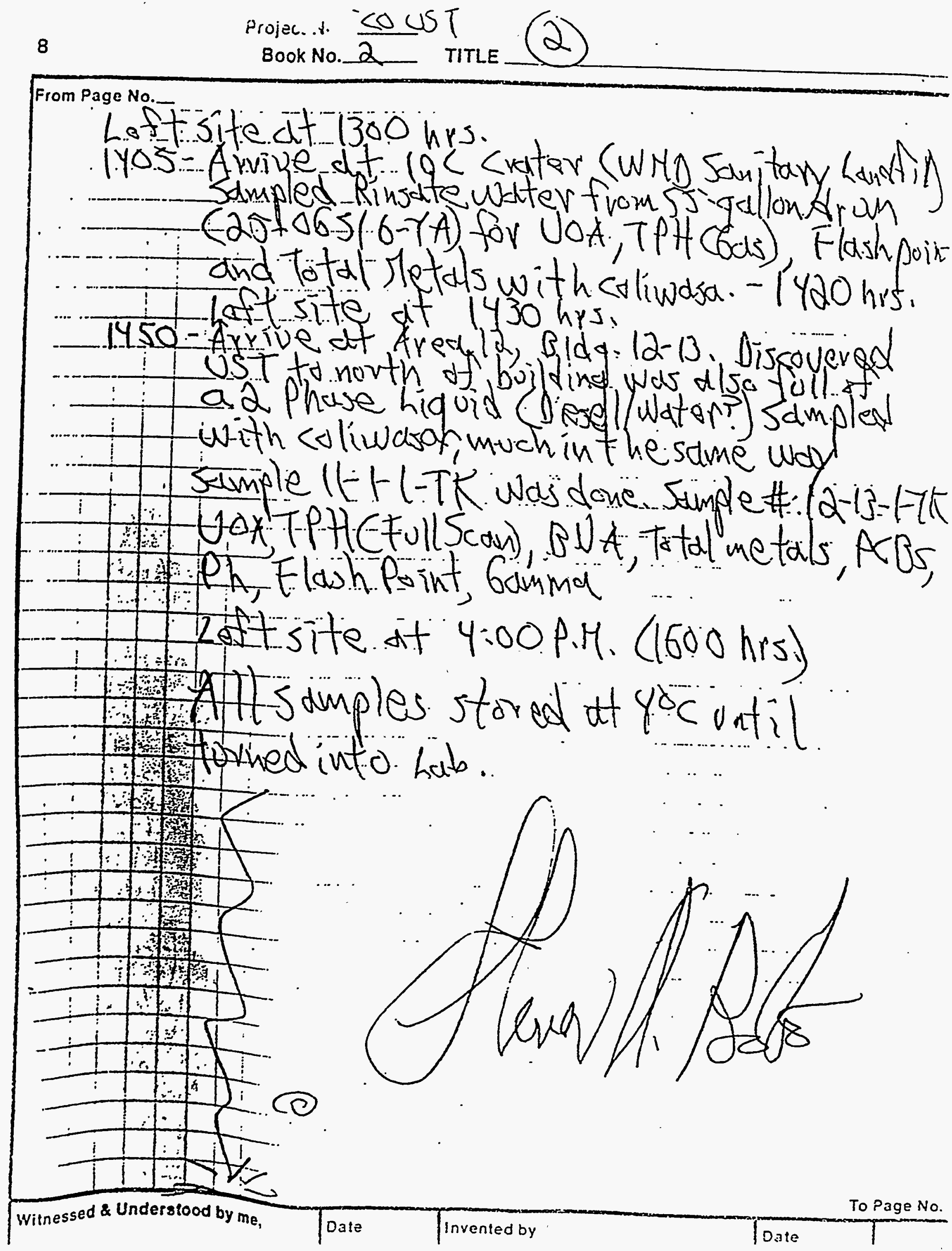




\section{Reynolds Electrical \& Engineerıng Co., Inc.}

\section{MEMORANDUM Git}

To .F. E. Bingham THRU L. S. Sygitowicz SUm for LSS

From G. A. Clark SNW for GAC

Date October 20, 1992

$\because$ : Subject SAMPLE ANALYTICAL RESULTS

Enclosed are DataChem's results for the total metals analyses of six oil/water samples collected on September 16, 1992, at the Area 11 Tweezer Facility. These analyses were requested by $D$. Madsen.

Please direct any questions you may have about these analyses to Sample Management (Roger Mitche11, 295-7220 or Jerry Dugas, 295-7997).

GAC:RNL667:rn

Enclosures

As . stated

cy: Central Files, w/o encls.

H. A. Hawthorne, w/o encls.

D. Madsen, w/encls

ACS Packet No. 92-09-049, W/O encls. 
REECO

FRAKK BINGHAH

ENVIRONHEHTAL COMPLIAHCE H/S 711

Attn: DAVE HADSEH

Purchase Order: 3754-009

Invoice Number:
Order *: 92-09-049

Date: 10/19/92 15:51

WOTK ID: TOTAL METALS/OIL; A11.

Oate Received: $09 / 17 / 92$

Date Completed: $10 / 12 / 92$

Client code: ENV_COMPLY

REPORT TO DAVE HADSEH, EHY_COHPLY.

SAHPLES SENT TO DATACHEH FOR AKALYSIS.

SEE DATACKEM \#S92-0710-AB FOR RESULTS.

\section{SAMPLE IDENTIFICATION}

Sample.

number

01

02

$11-1-1-T K$

$19-2-2-D R U H$
Sample

Description

Sample

Number

03

04

11-2-1-DRUM

$12-13-1-T K$

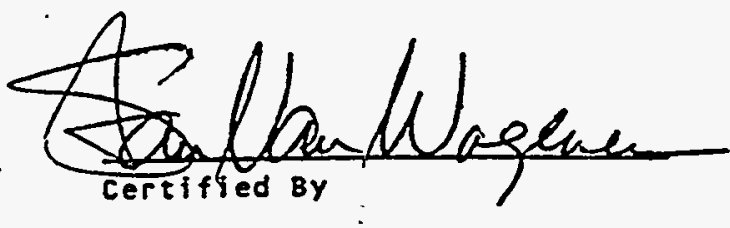


order $\$ 92-09-049$

Sample: 01A 11-1-1-TK

Test Description

SEHD OUT METAL ANALYSIS

Sample: 02A 11-2-2-DRUM

Test Description

SEND OUT METAL AHALYSIS

$\because$ :

Sample: 03A 11-2-1-DRUX

Test Description

SEHD OUT METAL AKALYSIS

Sample: 04A 12-13-1-TK

Iest Description

SEND OUT METAL ANALYSIS
REECO ASD/ACS

Collected: 09/16/92 Category: TOTAL_KETALS

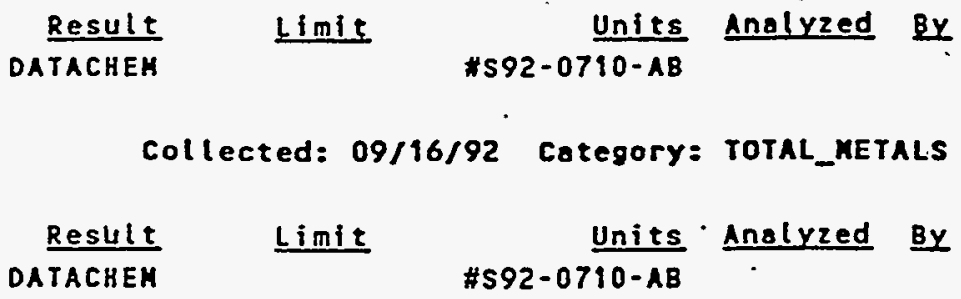

Collected: 09/16/92 Category: TOTAL_METALS

$\frac{\text { Result }}{\text { DATACHEM Limit }} \underset{\text { Units }}{\text { Analyzed }}$ By

Collected: 09/16/92 category: TOTAL_METALS

Result Limit Units Anolyzed By 
SEMD OUT KETAL AMRLYSIS Method: DATACHEM

Samp Sample Description

01A 11-1-1-TK

02A :11-2-2-DRUK

O3X 11-2-1-DRUH

$04 A \quad 12-13-9-T K$
Mininum: Naximure:

Result DATACHEM DATACHEY

DATACHEM

DATACHEM
Units Limit Prepared Analyzed $\underline{B y}$ \#S92-0710-AB

\#S92-0710-AB

\#S92-0710-AB

\#S92-0710-AB 
TOTAL METALS DATA 


\section{DATA

ENVIRONMENTAL WASTE REPORT

Form EPRG-A

REECO

$\because \mathrm{P}$. O. Box 98521, M/S 713

Las Vegas, NV 89193-8521

Attention: Jerry Dugas
Page 1 of 2

Part 1 of 1

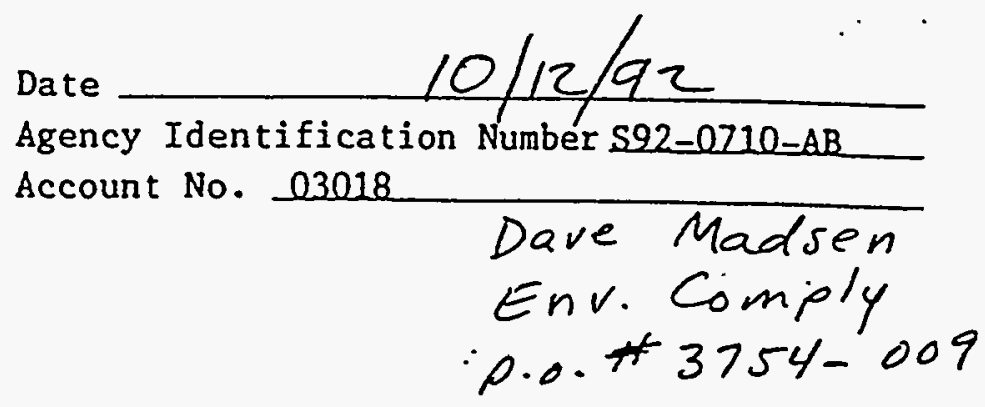

Telephone (702) 295-7997

Sampling collection and Shipment

Sampling Site $\mathrm{A}-11$

Date of Collection September $16,1^{\prime \prime n} 2$

Date Samples Received at Laboratory September 19, $1992^{\circ}$

Analytical Results

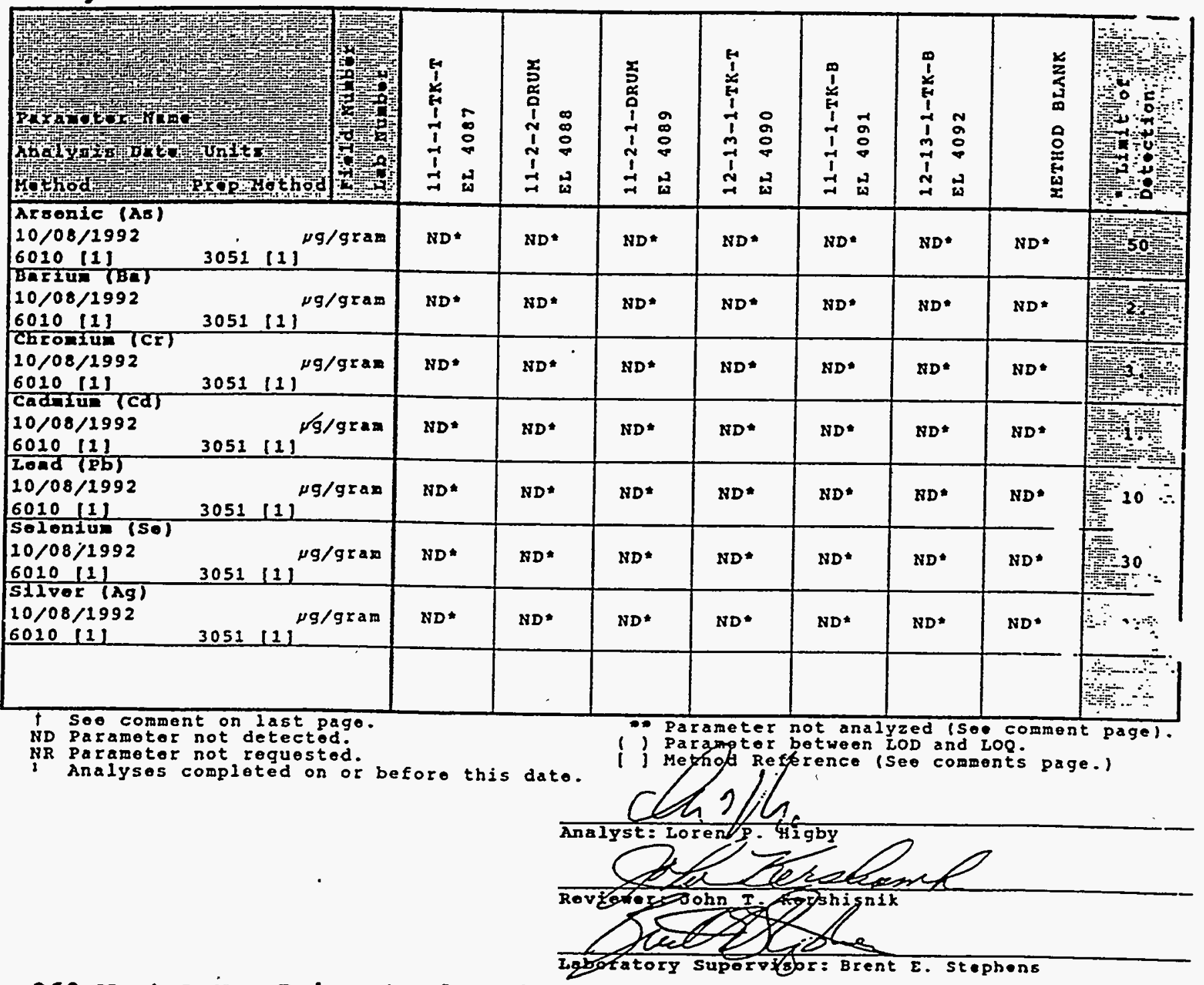

960 West Levoy Drive / Salt Lake City, Utah 84123-2547 / (801) 266-7700 A Sorenson Company 
Date $10 / 14 / 92$

Agency Identification Number $592-0710-B B$ Account No. 03018

REECO

$\because$ P. O. Box 98521 , M/S 713

Las Vegas, NV 89193-8521

Attention: Jerry Dugas

Telephone (702) 295-7997

Sampling Collection and Shipment

Sampling Site A-11

Date of Collection September 16, 1992

Date Samples Received at Laboratory September 19, 1992

Analytical Results

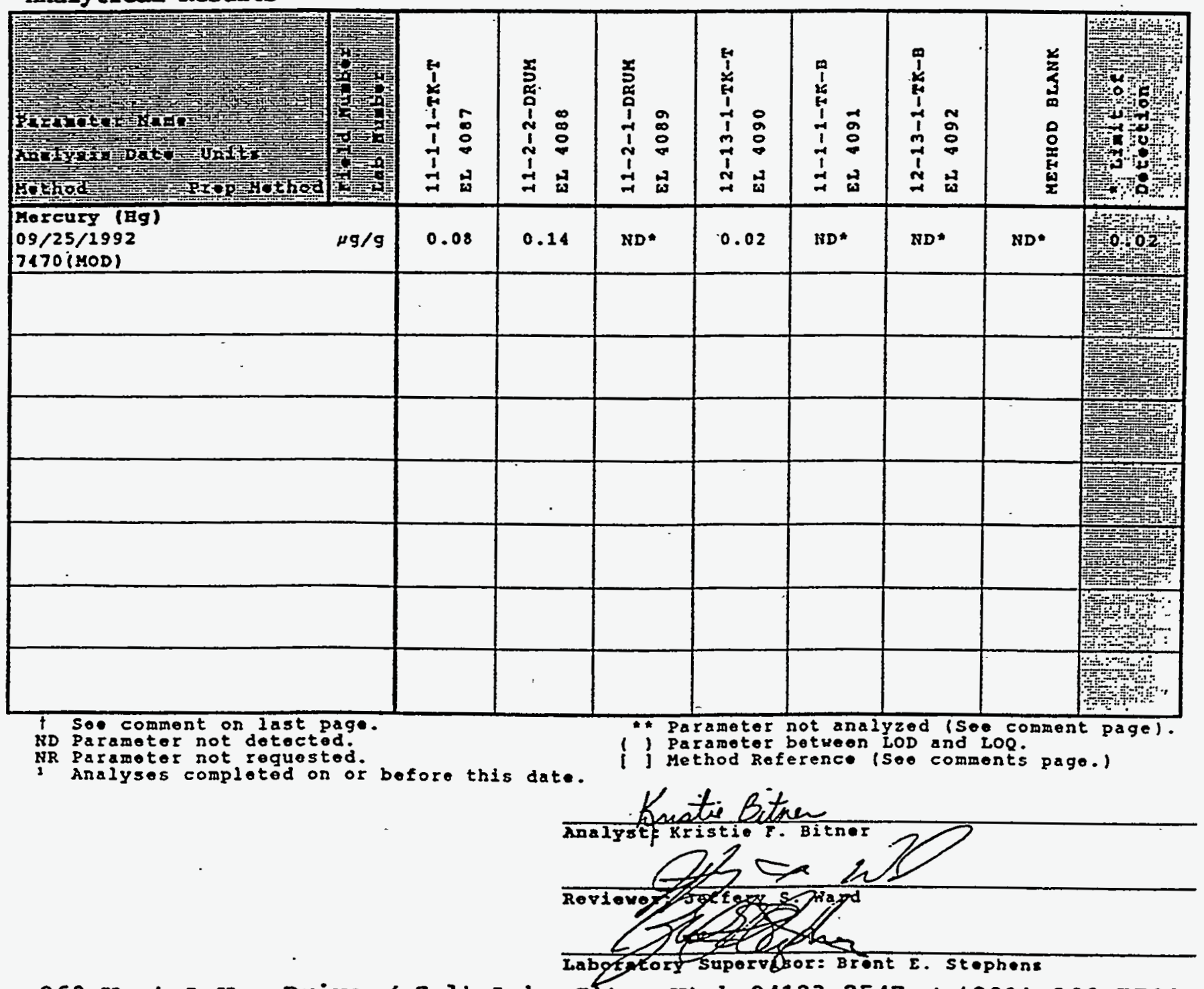

960 West LeVoy Drive / Salt Lake City, Utah 84123-2547/ (801) 266-7700 A Sorenson Company 
$\because \because{ }^{\circ}$

SUPPORTING DATA 
Reynurds Electrical \& Engineering Co., Inc.

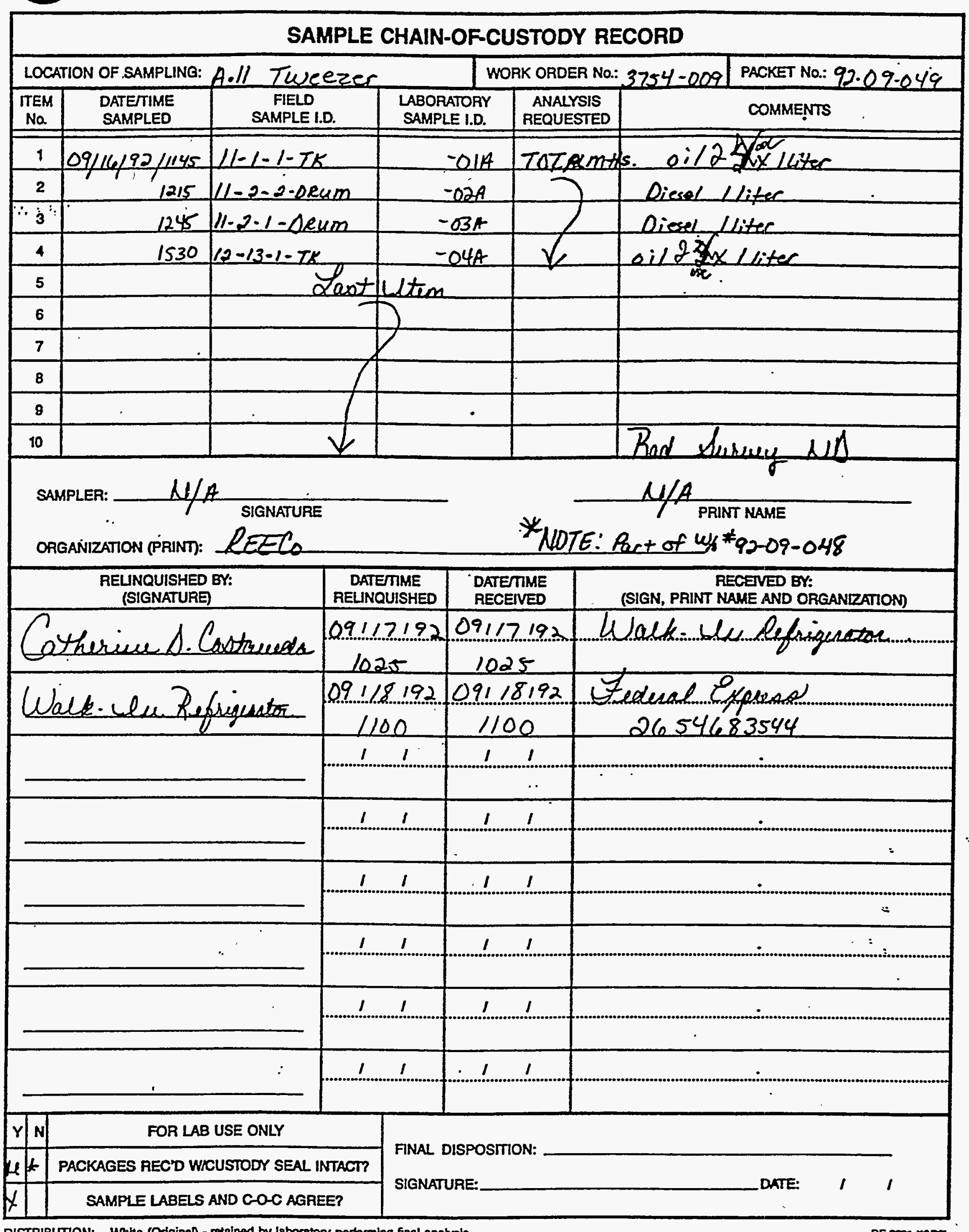

DISTRIBUTION: White (Original) - retained by laboratory performing final analysis

RE-2231 (12000) 
REYI. US ELECTRICAL AND ENGIA :HING COMPANY ANALYTICAL SERVICES DEPARIMENT

SAMPLE ANALYSIS REQUEST SHEET

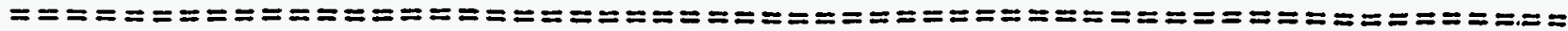

w.o.: $3754-009$

DATE SUBMITTED: $9 / 15 / 92$ DATE Reouired: $9 / 16 / 92$

REQUESTOR: D. D. MADSEN PHONE: $5-7211$ DEPT: ECO MIS: 711

sampung location: SeE Below contact: $4, P$. Lipstate phone: $5-3545$ area: SEe Belo

RAD ANALYSIS REQUIRED:

CHEMICAL ANALYSIS REQUIRED: YES

$\longleftarrow$ No PRIORITY CODE: RUSh:

TYPE REPORT REQUIRED:

DESCRIPTION OF WASTE:

X 1. TCL results only.

1. NUMBER OF SAMPLES:

2. TCL and OC rosults.

3. TCL and TIC rosults.

4. TCL, THC, and $\mathrm{DC}$ results.

2. TYPE(S) QF WASTE CONTANERS: $(3)$ lindergnound storage tenks
and ( $)$ SS gallon drum

5. CLP.TYPE Roport.

SPECIAL INSTRUCTONS: only one MS $+M S D$ do be be analyzed for batch, - sample Numbers t analyses resestud on brele-Note Fo-Datachem for sumples submitted for torel Metals Anolyses Conly need TCLP metals to be reported)

DATE REPORT IS REQUIRED: $10 / 1 / 92$ REQUESTOR SIGNATURE:

SAMPLER: $S \cdot$ Rolo

date collected: 9$)-16-9) 2$ ASD PACKET :

\begin{tabular}{|c|c|c|c|c|}
\hline$\angle A B I D$ & FIED SAMPLE : & MATRIX & SAMPLE SIZE & $\begin{array}{l}\text { ANALYSES REQUESTED } \\
\text { (METHOD \& REOUIRED) }\end{array}$ \\
\hline & 30 & $\frac{x_{2}+2}{2}$ & $2 \times 40 \mathrm{~m}$ & Ka Toin B) suth \\
\hline & & & & \\
\hline & & & & \\
\hline & & & & \\
\hline & & & & \\
\hline & & & & 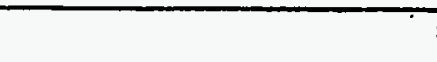 \\
\hline & & & & \\
\hline & & & & \\
\hline
\end{tabular}

COMMENTS: 


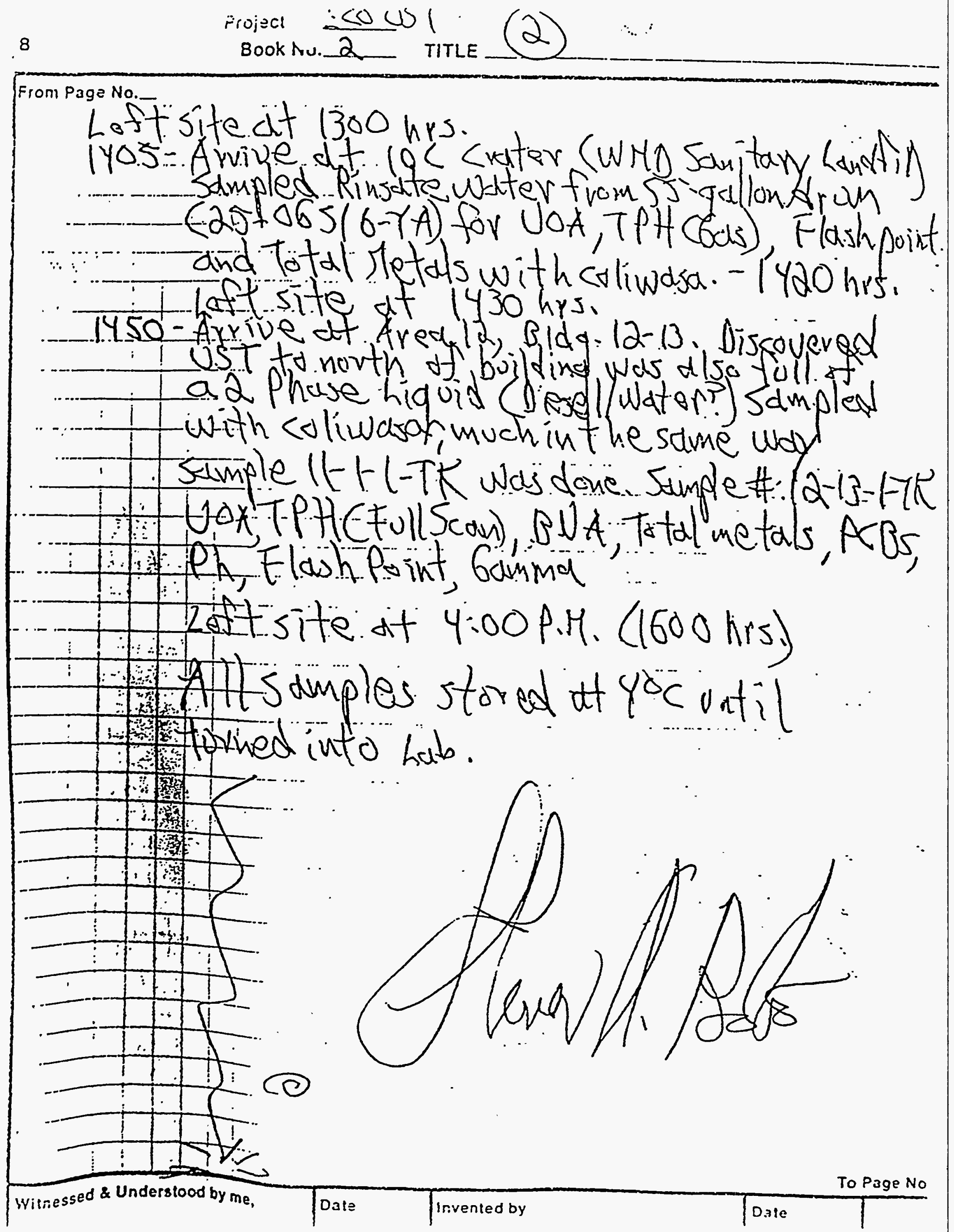




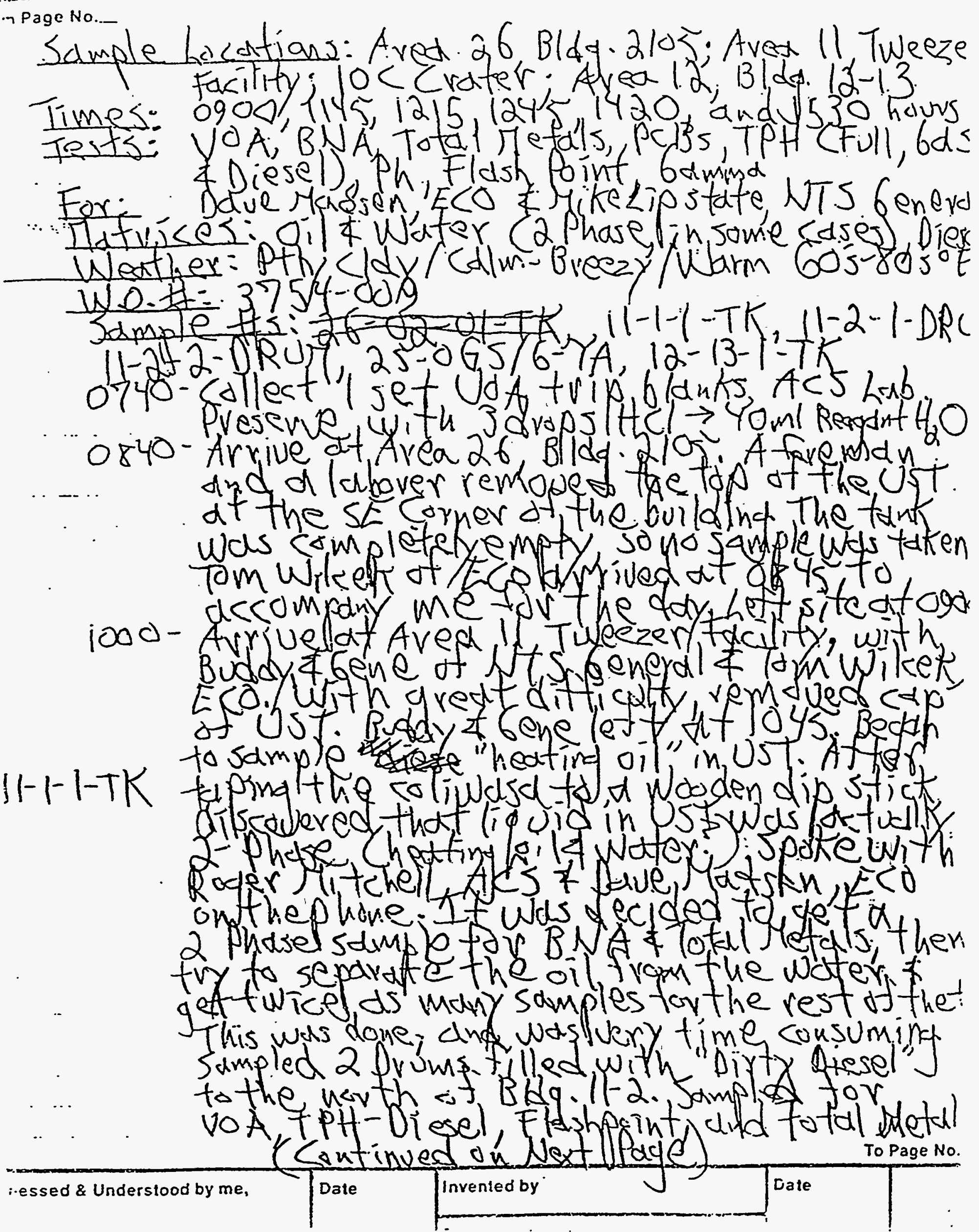




\section{Reynolds Electrical \& Engineering $\mathrm{CO}_{\approx}$ Inc.}

\section{MEMORANDUM}

$\begin{array}{ll}\text { To } & \text { S. J. Nacht } \\ \text { From } & \text { G. A. Clark Cote } \\ \text { Date } & \text { November } 17,1993 \\ \text { Subject } & \text { SAMPLE ANALYTICAL RESULTS }\end{array}$

Enclosed are Analytical Services Department's results, requested by T. A. Wilczek, for the total petroleum hydrocarbons (gasoline/diesel/oil) analyses of four soil and two water samples collected on October 20, 1993, in Areas 12 and 27. Also enclosed are the results for the gross gamma analysis of these samples.

Please direct any questions you may have about the nonradioanalytical results to Roger Mitchell (295-7220) or Jerry Dugas (295-7997), and questions about the radioanalytical results to Al Latham (295-7472).

GAC:RNL1277:rn

Enclosures

As stated

cy: Central Files, w/o encls.

A. R. Latham, w/o encls.

L. S. Sygitowicz, w/o encls.

T. A. Wilczek, w/encls:

ACS Packet No. 93-10-101, w/o encls. 


\section{REECO}

BOB DODGE

EHVIRONHENTAL RESTORATION TECHNOLOGY DEVELOPMENT

ATtN: TOH WILCZEK

Purchase Order: $3754-022$

Invoice Number:

REPORT TO TOM WILCZEK, ERTD order \#: 93-10-101

Date: 11/18/93 09:11

Hork ID: TPH/SOIL, HATER, PKT\#C005626

Date Received: $10 / 20 / 93$

Date Completed: $11 / 12 / 93$

Client Code: ERTD

SAMPLE IDENTIFICATION

\begin{tabular}{ll} 
Sample & \multicolumn{1}{c}{$\begin{array}{c}\text { Sample } \\
\text { Number }\end{array}$} \\
$\frac{\text { Duscription }}{01}$ & ERUSTP2/5170-E \\
02 & ERUSTP2/5170-H \\
03 & ERUSTP2/5170-RINSE \\
04 & ERUSTP2/1213-H \\
05 & ERUSTP2/1213-E \\
06 & ERUSTP2/1213-RIHSE \\
07 & $93-10-101-Q C 1 \quad$ HS/SOIL
\end{tabular}

Sample

Number

Sample

08

Description

09

93-10-101-QC2 BLANK/SOIL

93-10-101-QC3 CAL VER/SOIL

$93-10-101-0 C 4$

MS/WATER

11

93-10-101-QC5 BLANK/HATER

12

93-10-101-QC6 CALVER/HATER

PACKET \#C005626

14 PACKET \#C005626

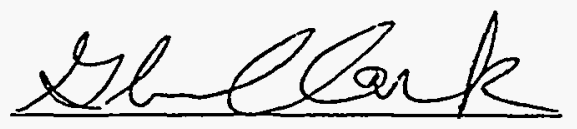

Certified $8 y$ 
RADIOISOTOPE SCREEH

He thod:

Samp Sample Description

13A PACKET \#C005626

RADIOHUCLIDE FIHAL REPORT

Method:

Samp Sample Description

$14 \mathrm{~A}$ PACKET \#C005626

TPH - DIESEL

Hethod: GC

Samp Sample Description

O1A ERUSTP2/5170-E

O2A ERUSTP2/5170-H

03A ERUSTP2/5170-RINSE

04A ERUSTP2/1213-W

05A ERUSTP2/1213-E

O6A ERUSTP2/1213-RINSE

07A 93-10-101-QC1 HS/SOIL

08A 93-10-101-QC2 BLANK/SOIL

09A 93-10-101-QC3 CAL VER/SOIL

10A 93-10.101-0C4 MS/WATER

11A 93-10-101-0C5 BLANK/WATER

12A'93-10-101-QC6 CALVER/WATER

TPH - GASOLINE

Method: GC

Samp Sample Description

01A ERUSTP2/5170-E

02A ERUSTP2/5170-H

03A ERUSTP2/5170-RINSE

04A ERUSTP2/1213-H

05A ERUSTP2/1213-E

O6A ERUSTP2/1213-RINSE

07A 93-10-101-AC1 MS/SOIL

08A 93-10-101-QC2 BLANK/SOIL

09A 93-10-101-QC3 CAL VER/SOIL

10A 93-10-101-QC4 HS/WATER

11A 93-10-101-QC5 BLANK/HATER

12A 93-10-101-QC6 CALVER/WATER
Hinimum:

Result SEE DATA

Minimum:

Result SEE DATA

Hinimum:

Result

816

471

2.20

$<10.0$

116

0.730

92

$<10.0$

190

NA

$<0.5$

76

Minimum:

Result

$<5.0$

$<5.0$

$<5.0$

$<5.0$

$<5.0$

$<5.0$

171

< 5.0

101

119

$<5.0$

101
Maximum:

Units Limit Prepared Analyzed By PACKAGE

O Haximum: 999999999

Units Limit Prepared Analyzed By PACKAGE

o Maximum: 999999999

Units Limit Prepared Analyzed By $\mathrm{mg} / \mathrm{Kg} \quad 11 / 04 / 93 \mathrm{TJR}$ $\mathrm{mg} / \mathrm{Kg} \quad 11 / 04 / 93 \mathrm{TJR}$ $\mathrm{mg} / \mathrm{L} \quad 11 / 03 / 93 \mathrm{TJR}$ $\mathrm{mg} / \mathrm{Kg} \quad 11 / 04 / 93 \mathrm{TJR}$ $\mathrm{mg} / \mathrm{Kg} \quad 11 / 04 / 93 \mathrm{TJR}$ $11 / 03 / 93 \mathrm{TJR}$ $11 / 04 / 93$ TJR $11 / 04 / 93 \mathrm{TJR}$ $11 / 04 / 93 \mathrm{TJR}$ TJR $11 / 03 / 93$ TJR $11 / 03 / 93$ TJR

o Haximum: 999999999

Units Limit Prepared Analyzed By $\mathrm{mg} / \mathrm{Kg} \quad 11 / 02 / 93 \mathrm{TJR}$ $\mathrm{mg} / \mathrm{Kg} \quad 11 / 02 / 93 \mathrm{TJR}$ $\mathrm{mg} / \mathrm{L} \quad 11 / 02 / 93 \mathrm{TJR}$ $\mathrm{mg} / \mathrm{Kg} \quad 11 / 02 / 93 \mathrm{TJR}$ $\mathrm{mg} / \mathrm{Kg} \quad 11 / 02 / 93 \mathrm{TJR}$ $\mathrm{mg} / \mathrm{L} \quad 11 / 02 / 93 \mathrm{TJR}$ \% RECOVERY $\mathrm{mg} / \mathrm{Kg}$

\% RECOVERY

\% recovery $\mathrm{mg} / \mathrm{L}$

\% recovery
$11 / 02 / 93$ TJR

11/02/93 TJR

$11 / 02 / 93 \mathrm{TJR}$

$11 / 02 / 93 \mathrm{TJR}$

$11 / 02 / 93 \mathrm{TJR}$

$11 / 02 / 93$ TJR 
Order \# 93-10-101

$11 / 18 / 9309: 11$
TPH - OIL

Hethod: GC

Samp Sample Description

01A ERUSTP2/5170-E

O2A ERUSTP2/5170-W

O3A ERUSTP2/5170-RINSE

O4A ERUSTP2/1213-H

05A ERUSTP2/1213-E

O6A ERUSTP2/1213-RINSE

O7A 93-10-101-OC1 MS/SOIL

08A 93-10-109-QC2 BLANK/SOIL

09A 93-10-101-QC3 CAL VER/SOIL

$10 \mathrm{~A}$ 93-10-101-QC4 . HS/HATER

11A 93-10-101-0C5 BLANK/WATER

12A 93-10-101-QC6 CALVER/WATER

TPH - TOTAL

Hethod = GC

Samp Sample Description

O1A ERUSTP2/5170-E

D2A ERUSTP2/5170-W

O3A ERUSTP2/5170-RINSE

O4A ERUSTP2/1213-W

O5A ERUSTP2/1213-E

O6A ERUSTP2/1213-RINSE

08A 93-10-101-0C2 BLANK/SOIL

11A 93-10-101-QC5 BLANK/WATER
Hinimum:

Result

$<10.0$

$<10.0$

$<0.5$

$<10.0$

$<10.0$

$<0.5$

39

$<10.0$

114

NA

$<0.5$

77

Minimum:

Result

816

473

2.20

$<25.0$

116

0.730

$<25.0$

$<6.0$ o Haximum: 999999999

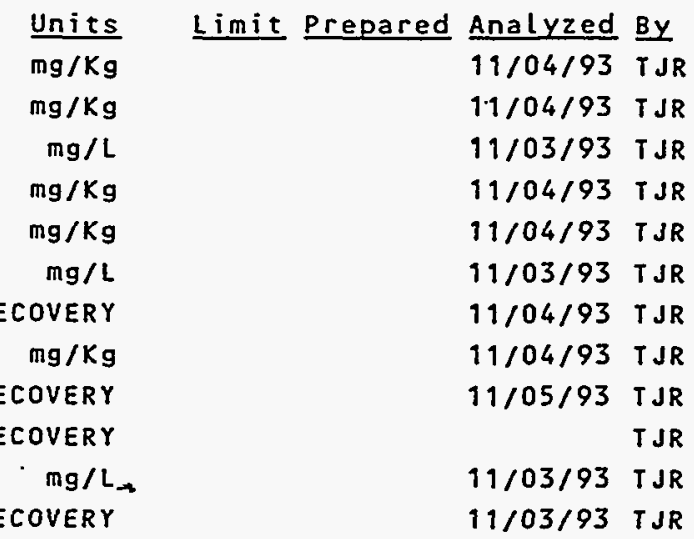

0 Maximum: 999999999

Units $\quad$ Limit Prepared Analyzed By
$\mathrm{mg} / \mathrm{Kg}$
$\mathrm{mg} / \mathrm{Kg}$
$\mathrm{mg} / \mathrm{L}$
$\mathrm{mg} / \mathrm{Kg}$
$\mathrm{mg} / \mathrm{Kg}$
$\mathrm{mg} / \mathrm{L}$
$\mathrm{mg} / \mathrm{Kg}$
$\mathrm{mg} / \mathrm{L}$


TPH Analyst's Notes:

All analyses of the sample extracts, with the exception of ERUSTP2/1213-H, produced chromatograms indicating the presence of a component whose reponse maximum lies between that seen for the Diesel and oil standards. However, as the geometry of the chromatogram is similar to that of Diesel, the Diesel calibration was used to quantitate the component. The reported values should be viewed as rough estimates. of the contaminant present.

Analysis of the aqueous extracts was performed on the 3 rd of November. The * analyses of the Diesel and 0 il Calibration Verification standards on that column returned at approximately $75 \%$ of their expected values. The results for those samples were not adjusted.

The aqueous $D i e s e l$ and $0 i l$ matrix spikes were extracted from samples of other work orders which were highly contaminated and therefore did not allow for the recovery of the spikes.

The soil oil matrix spike was prepared from a sample of another set which was highly contaminated with Diesel and $0 i l$. The spike could not be seen due to these levels. 

s

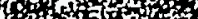
H (1)

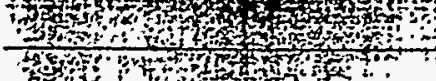

- B.t.

(c)

Fin.

70

2. $\rightarrow 57:$

$\because$

a

:

$=$

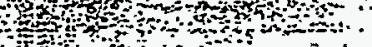

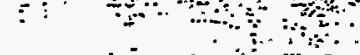

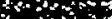
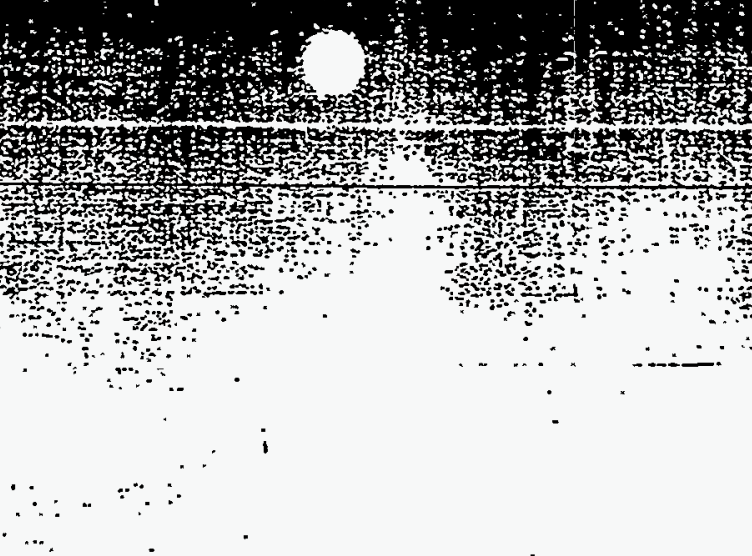

SUPPORTING DATA

$\vdots$
$\vdots$
$\vdots$
$\vdots$
$\vdots$
$\vdots$ 



\section{Reynolds Electrical \& Engineering Co., Inc.}

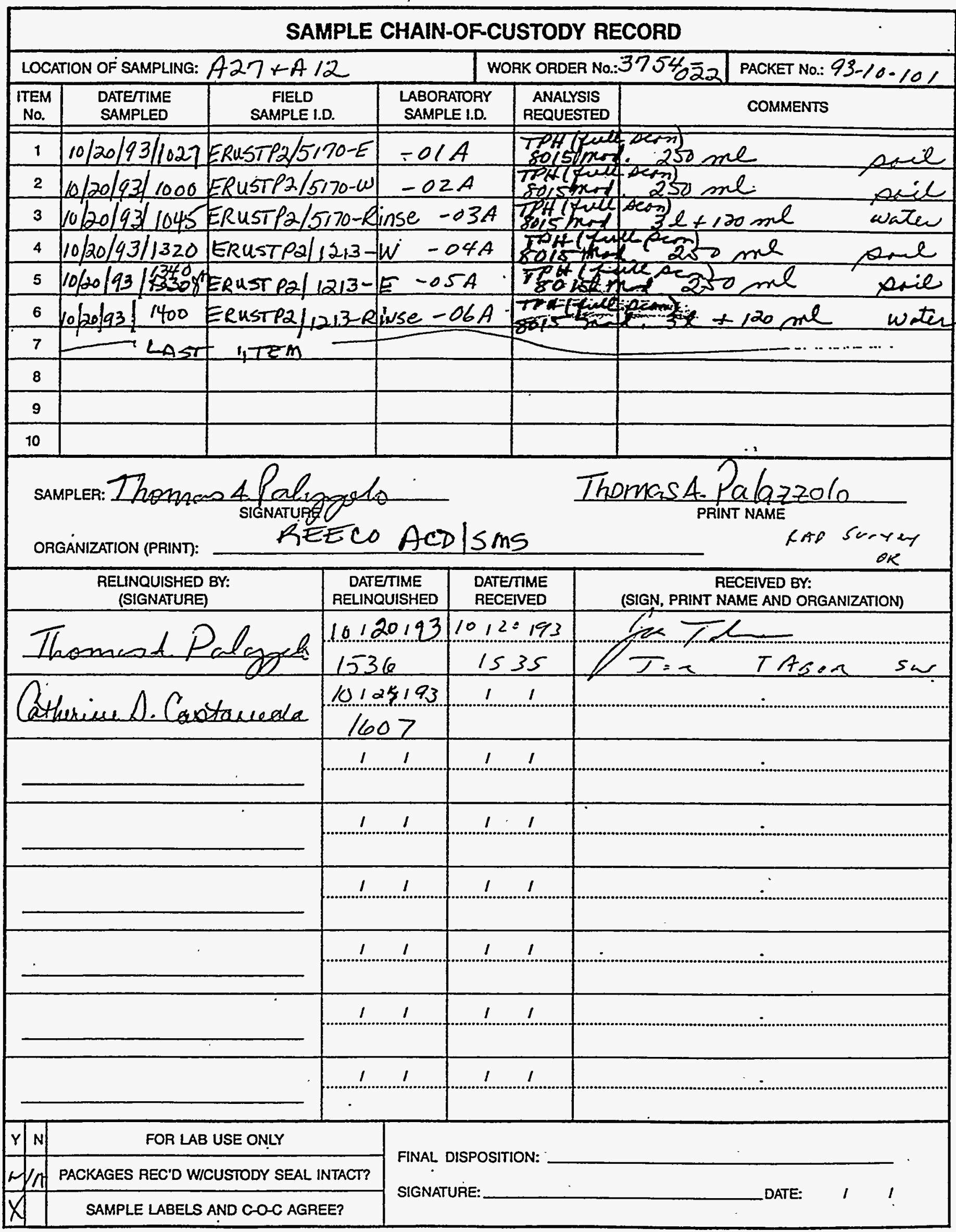


REYNC JS ELECTRICAL AND ENGINEE. .NG COMPANY ANALYTICAL SERVICES DEPARTMENT

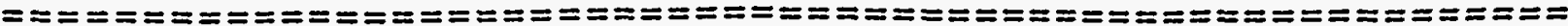

W.o.*: $3754-022$ DATE SUBMITTED: $10 / 4 / 93$ DATE REOUIRED: $10 / 20 / 93$

ReQuestor: Tom WilczeK $A / 27-86 j \mathrm{~s} 5170$ SAMPLNG LOCATION: Af12-bLdS $12 \cdot 13$ PHONE: 5396 CONTACT: SAME DEPT: ERS Mis: 966 PHONE: SAME AREA: 23

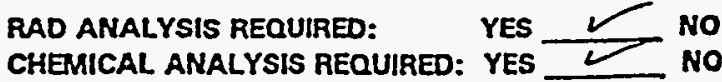
NO__ PRIORITY CODE: 4-RUSAf PRIORITY: NORMal

TYPE REPORT REQUIRED:

1. TCL results only.

2. TCL and $\mathrm{QC}$ results.

3. TCL and TIC results.

4. TCL, TIC, and $\mathrm{CC}$ results.

5. CLP.TYPE Report.
DESCRIPTION OF WASTE:

1. NUMBER OF SAMPLES: 4 -Soils, 2-RIuse, 6-head SPACE

2. TYPE(S) OF WASTE CONTAINERS: Soil $t$ head space saviles feom puds of Two SeParate UST'S And 2 Sondes From SepaEATE DECON RINSE WATER

Speclal instauctions: Sampler to Brina piD For HeadsofeE Ana Lssis. Be at A/27

Sout Gate e - 8:30/8:45. Tauk yauk to Besin e 9:00a. Expect smplin to cart All DAY. All2 Yawk to Be accomplished sare day if enough tine Aflows.

Prelims: "1/10/93

DATE REPORT IS REQUIRED: FINAC: $11 / 1 / 9 / 93$ REQUESTOR SIGNATURE:

Thomas C. Lielese

f sampler: ASD PACKET :

\begin{tabular}{|c|c|c|c|c|}
\hline LAB ID * & FIEDD SAMPLE \# & MATRIX & SAMPLE SIZE & $\begin{array}{l}\text { ANALYSES REQUESTED } \\
\text { (METHOD \# REQUIRED) }\end{array}$ \\
\hline & ERUSTPR/5170-E & Soil & $250 \mathrm{ml}$ & TPH ( full scan $) 8015 \mathrm{Mod}$ \\
\hline & ERUSTP2/5170-W & Soil & $250 \mathrm{ml}$ & $T P H($ full $\left.) 8015 M_{\text {sLd }}\right) 8$ \\
\hline & ERUST P2/5/70-RINSE & water & $3 l+120 \mathrm{ml}$ & 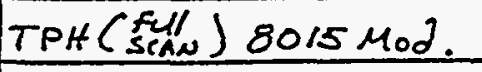 \\
\hline & 11 & 81 & $500 \mathrm{ml}$ & Gamma Scan. \\
\hline & ERUST P2/1213-NEPLTW & Soil & $250 \mathrm{ml}$ & TPH $\left(\right.$ fUll $\left._{S C A N}\right) 8015 \mathrm{MOd}$ \\
\hline & EqUSTP2/12/3-\$w N & Soil & $250 \mathrm{~mol}$ & TPH (fuly scAN $) 8015$ Mod.' \\
\hline & ERUST $P 2 / 1213-R_{\text {INSE }}$ & water & $3 l+120 \mathrm{ml}$ & $T P H($ full $)$ sean $) 8015 M_{00}$. \\
\hline & $\therefore$ & $"$ & $5 \pi \mathrm{mll}$ & Gamma Scan \\
\hline
\end{tabular}

COMMENTS: 


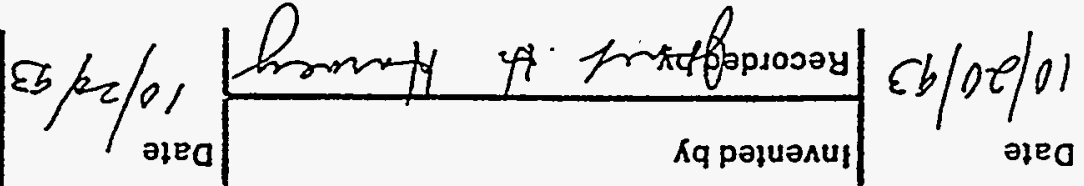

'әแ אq poolssəpuก จ pəssəu!!M!

- ON $26 \mathrm{C}_{\mathrm{d}}$ OI

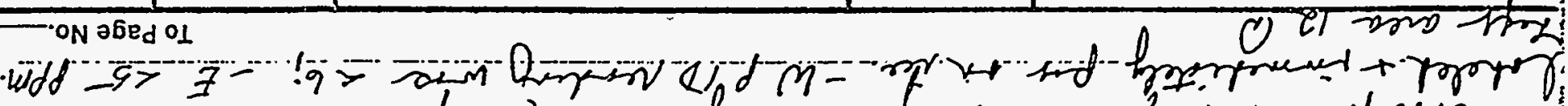

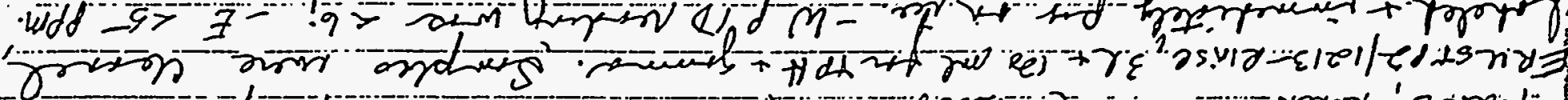

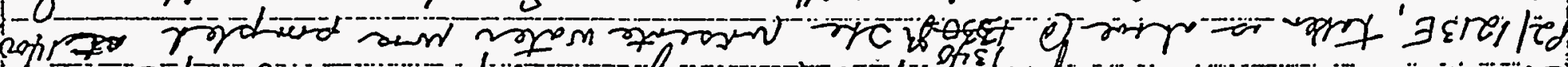

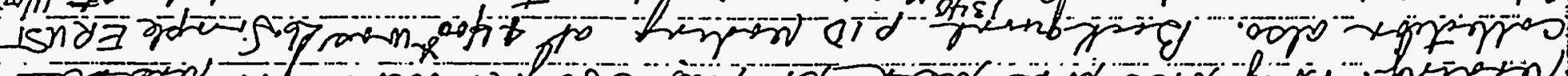

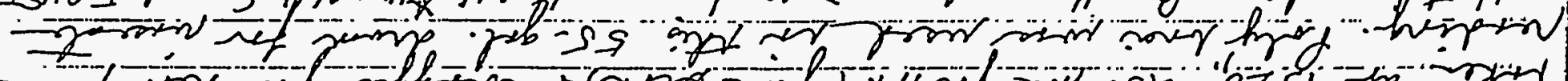

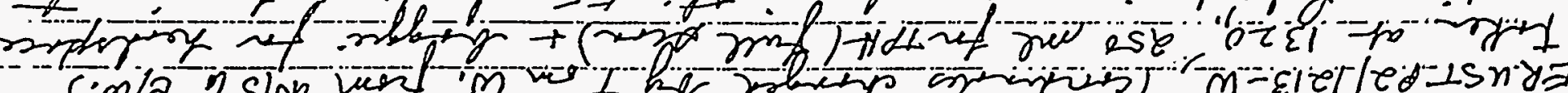

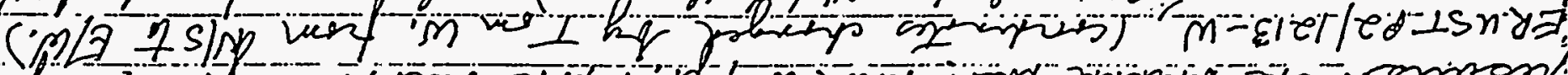

riluis

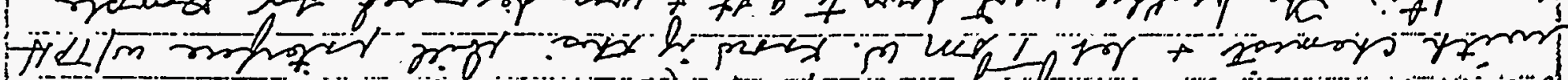

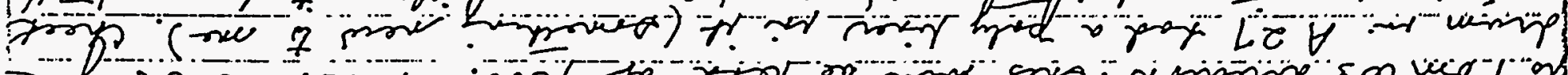

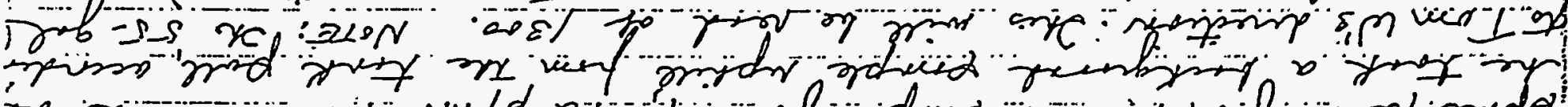

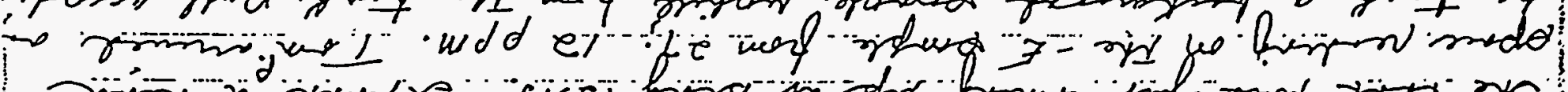

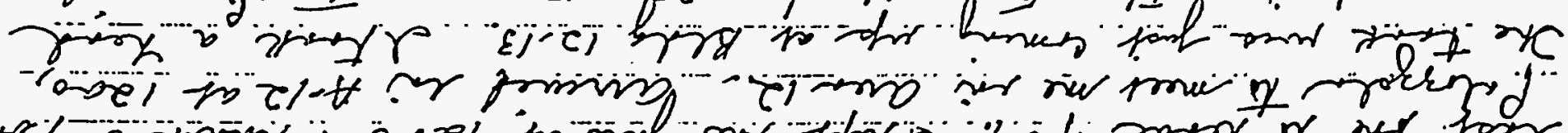

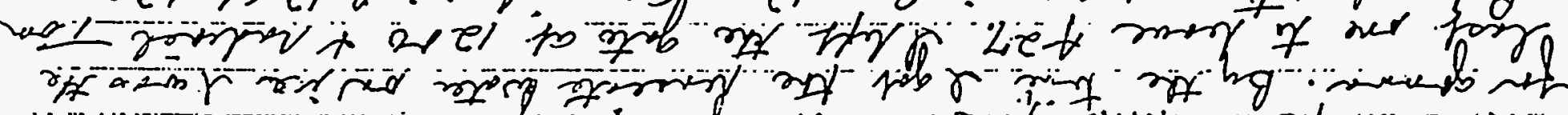

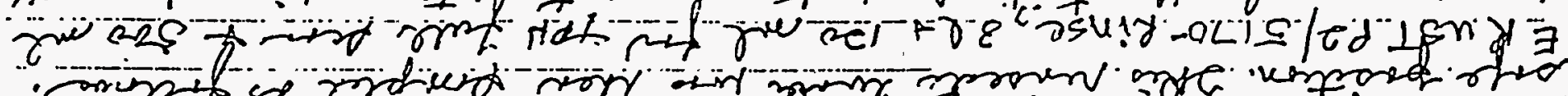

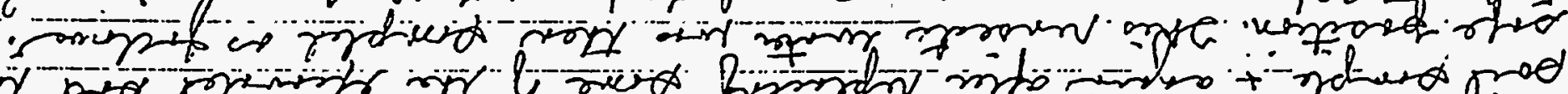

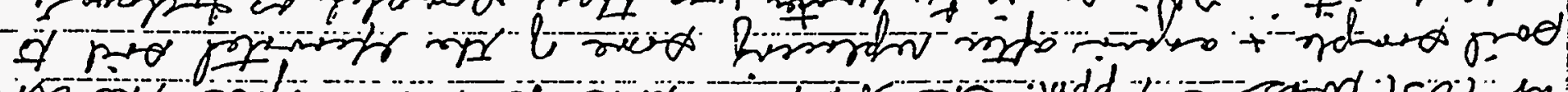

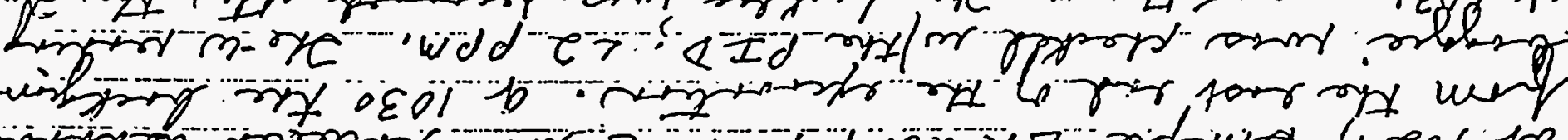
1

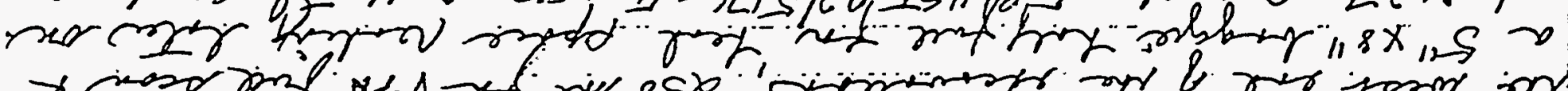

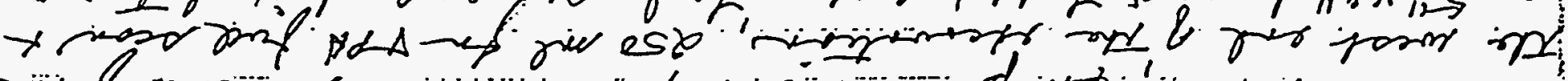

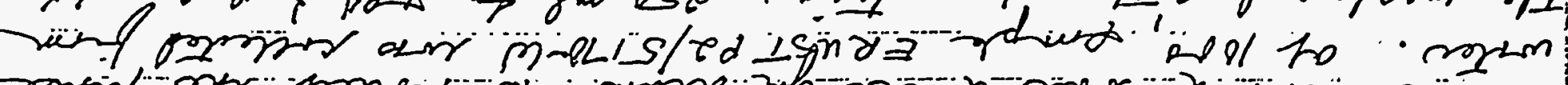

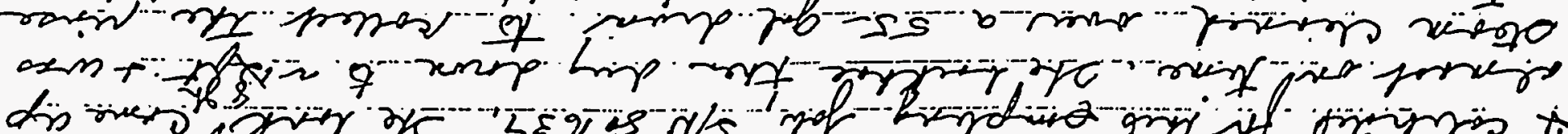

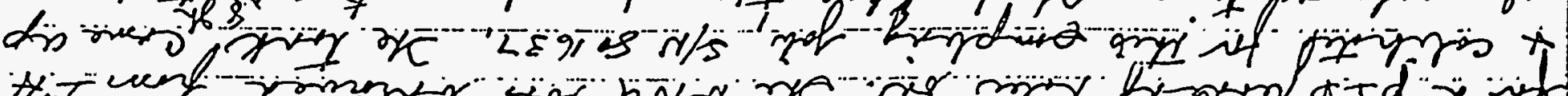

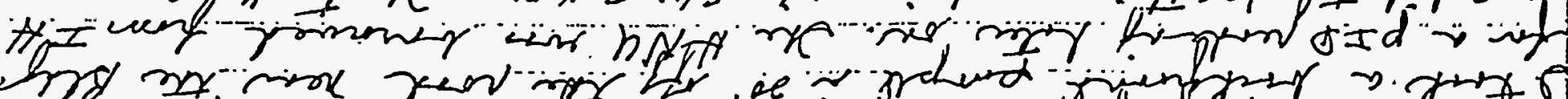

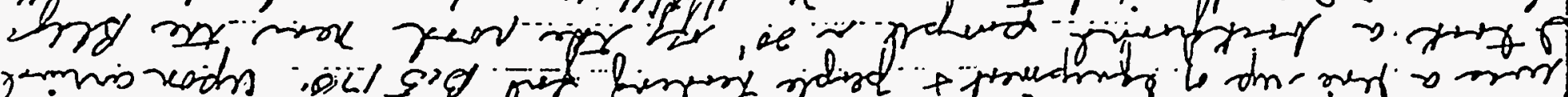

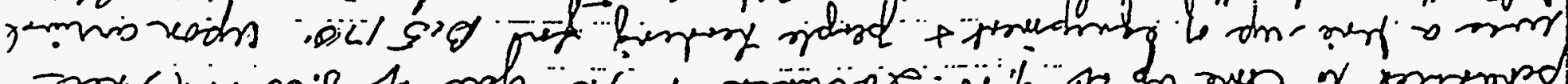

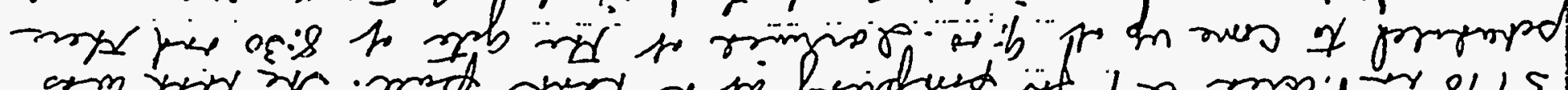

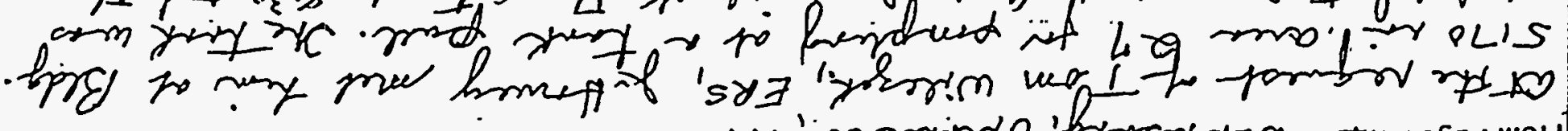

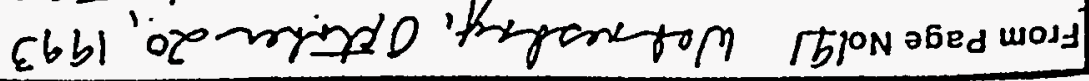


Reynolds Electrical \& Engineering Co., Inc.

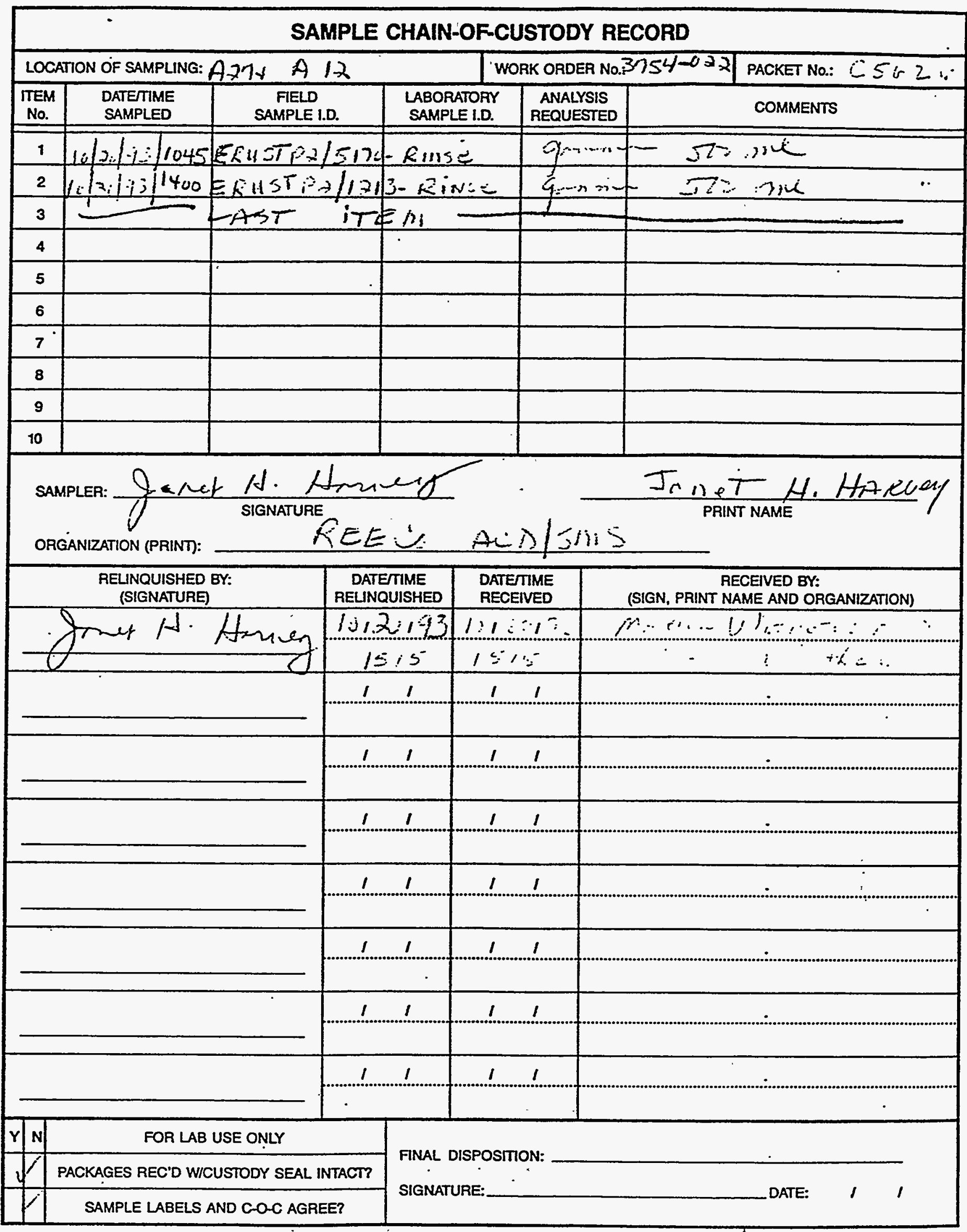

$\begin{array}{ll}\text { DISTRIBUTION: } & \text { White (Original) - retained by laboratory performing final analysis } \\ \text { Yellow - retained by laboratory performing intermediate analysis }\end{array}$

RE.2231 (121299) 


\section{Reynolds Electrical \& Engineering Co.,Inc.}

\section{MEMORANDUM}

To

S. J. Nacht

From

G. A. Clark Gote

Date

December 9, 1993

Subject

SAMPLE ANALYTICAL RESULTS

s

Enclosed are Analytical Services Department's results, requested by T. A. Wilczek, for the total petroleum hydrocarbons (gasoline/diesel/oil) analyses of seven soil samples collected on November 9, 1993, in Areas 12, 23, 25, ydind 27.

Please direct any questions you may have about these results to Roger Mitchell (295-7220) or Jerry Dugas (295-7997).

GAC:RNL1326:rn

Enclosures

As stated

cy: Central Files, w/o encls.

A. R. Latham, w/o encls.

L. S. Sygitowicz, w/o encls.

T. A. Hilczek, w/encls.

ACS Packet No. 93-11-051, w/o encls. 
REECO

BOB DODGE

ENVIRONMENTAL RESTORATION

TECHNOLOGY DEVELOPMENT

Attn: TOM WILCZEK

Purchase Order: 3754-022

Invoice Number:

REPORT TO TOM HILCZEK, ERTD.

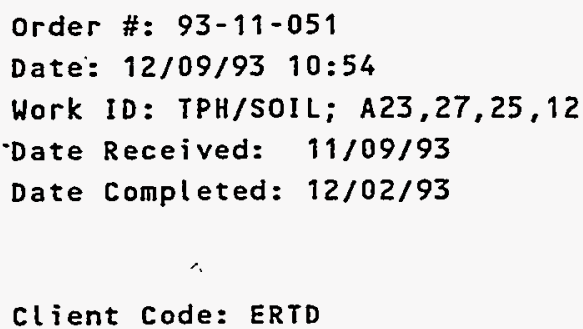

SAMPLE IDENTIFICATION

\begin{tabular}{ll}
$\begin{array}{l}\text { Sample } \\
\text { Number }\end{array}$ & \multicolumn{1}{c}{$\begin{array}{c}\text { Sample } \\
\text { Description }\end{array}$} \\
01 & $-23-110 /$ PILE \\
02 & $23-112 /$ PILE \\
03 & $12-8 /$ PILE \\
04 & $12-9 /$ PILE \\
05 & $12-13 /$ PILE \\
06 & $25-3205 /$ PILE
\end{tabular}
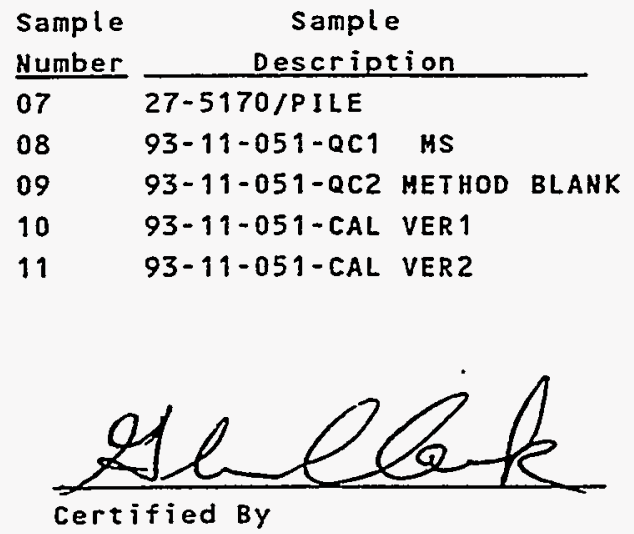
TPH - DIESEL

Hethod: GC

Samp Sample Description

01A 23-110/PILE

02A 23-112/PILE

03A. 12-8/PILE

04Aं 12-9/PILE

05A 12-13/PILE

06A 25-3205/PILE

07A 27-5170/PILE

08A 93-11-051-QC1 HS

09A 93-11-051-QC2 METHOD BLANK

10A 93-11-051-CAL VER 1

11A 93-11-051-CAL VER2

TPH - GASOLINE

Method: GC

Samp Sample Description

\begin{tabular}{ll}
\hline $01 A$ & $23-110 / P I L E$ \\
02A & $23-112 / P I L E$ \\
03A & $12-8 / P I L E$ \\
04A & $12-9 / P I L E$ \\
05A & $12-13 / P I L E$ \\
06A & $25-3205 / P I L E$ \\
07A & $27-5170 / P I L E$ \\
09A & $93-11-051-Q C 2$ METHOD BLANK \\
$10 A$ & $93-11-051-$ CAL VER1
\end{tabular}

TPH - GASOLIHE MATRIX SPK

Method: GC

$\begin{array}{ll}\frac{\text { Samp }}{01 A} & \frac{\text { Sample Description }}{23-110 / P I L E} \\ 02 A & 23-112 / P I L E \\ 03 A & 12-8 / P I L E \\ 04 A & 12-9 / \text { PILE } \\ \text { 05A } & 12-13 / \text { ILE } \\ \text { 06A } & 25-3205 / P I L E \\ \text { 07A } & 27-5170 / P I L E\end{array}$

TPH - OIL

Hethod: GC

Samp Sample Description

$\begin{array}{ll}\text { OIA } & 23-110 / P I L E \\ \text { O2A } & 23-112 / P I L E \\ 03 A & 12-8 / P I L E \\ 04 A & 12-9 / P I L E\end{array}$

Hinimum:

O Maximun: 999999999

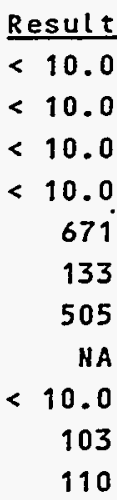

Minimum:

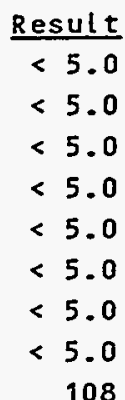

Minimum:

Result

113

115

142

113

133

99

134

Hinimun:

Result 70.4

$<10.0$

- 144

$<10.0$

o Maximum: 9999999.99

o Maximum: 999999999 \% RECOVERY
\% RECOVERY
$\%$ RECOVERY
$\%$ RECOVERY
$\%$ RECOVERY
$\%$ RECOVERY
$\%$ RECOVERY

o Maximum: 999999999

\begin{tabular}{|c|c|c|c|c|}
\hline & Units & Limit Prepared & Analyzed & By \\
\hline & $\mathrm{mg} / \mathrm{Kg}$ & & $11 / 23 / 93$ & $T J R$ \\
\hline & $\mathrm{mg} / \mathrm{Kg}$ & & $11 / 23 / 93$ & TJR \\
\hline & $\mathrm{mg} / \mathrm{Kg}$ & & $11 / 24 / 93$ & $T J R$ \\
\hline & $\mathrm{mg} / \mathrm{Kg}$ & & $11 / 23 / 93$ & $T J R$ \\
\hline & $\mathrm{mg} / \mathrm{Kg}$ & & $11 / 23 / 93$ & $T J R$ \\
\hline & $\mathrm{mg} / \mathrm{Kg}$ & & $11 / 23 / 93$ & $T J R$ \\
\hline $\mathrm{mg} / \mathrm{Kg}$ & (TEXT) & & $11 / 24 / 93$ & TJR \\
\hline$\%$ RE & COVERY & & & \\
\hline & $\mathrm{mg} / \mathrm{Kg}$ & & $11 / 23 / 93$ & $T J R$ \\
\hline$\%$ RE & COVERY & & $11 / 23 / 93$ & TJR \\
\hline$\%$ RE & COVERY & & $11 / 24 / 93$ & T JR \\
\hline
\end{tabular}

Units Limit Prepared Analyzed By $\mathrm{mg} / \mathrm{Kg} \quad 11 / 19 / 93 \mathrm{TJR}$ $\mathrm{mg} / \mathrm{Kg} \quad 11 / 19 / 93 \mathrm{TJR}$ $\mathrm{mg} / \mathrm{Kg} \quad 11 / 19 / 93 \mathrm{TJR}$ $\mathrm{mg} / \mathrm{Kg} \quad 11 / 19 / 93 \mathrm{TJR}$ $\mathrm{mg} / \mathrm{Kg} \quad 11 / 19 / 93 \mathrm{TJR}$ $\mathrm{mg} / \mathrm{Kg} \quad 11 / 19 / 93 \mathrm{TJR}$ $\mathrm{mg} / \mathrm{kg} \quad 11 / 20 / 93 \mathrm{TJR}$ $\mathrm{mg} / \mathrm{kg} \quad 11 / 19 / 93 \mathrm{TJR}$ \% RECOVERY 11/20/93 TJR

Units Limit Prepared Analyzed By 11/19/93 TJR $11 / 19 / 93 \mathrm{TJR}$ $11 / 19 / 93 \mathrm{TJR}$ $11 / 19 / 93 \mathrm{TJR}$ $11 / 19 / 93 \mathrm{TJR}$ $11 / 20 / 93 \mathrm{TJR}$ $11 / 20 / 93 \mathrm{TJR}$

Units Limit Prepared Analyzed By $\mathrm{mg} / \mathrm{Kg}$ (TEXT) . 11/23/93 TJR $\mathrm{mg} / \mathrm{Kg} \quad 11 / 23 / 93 \mathrm{TJR}$ $\mathrm{mg} / \mathrm{Kg} \quad 11 / 24 / 93 \mathrm{TJR}$ $\mathrm{mg} / \mathrm{kg} \quad 11 / 23.193 \mathrm{TJR}$ 


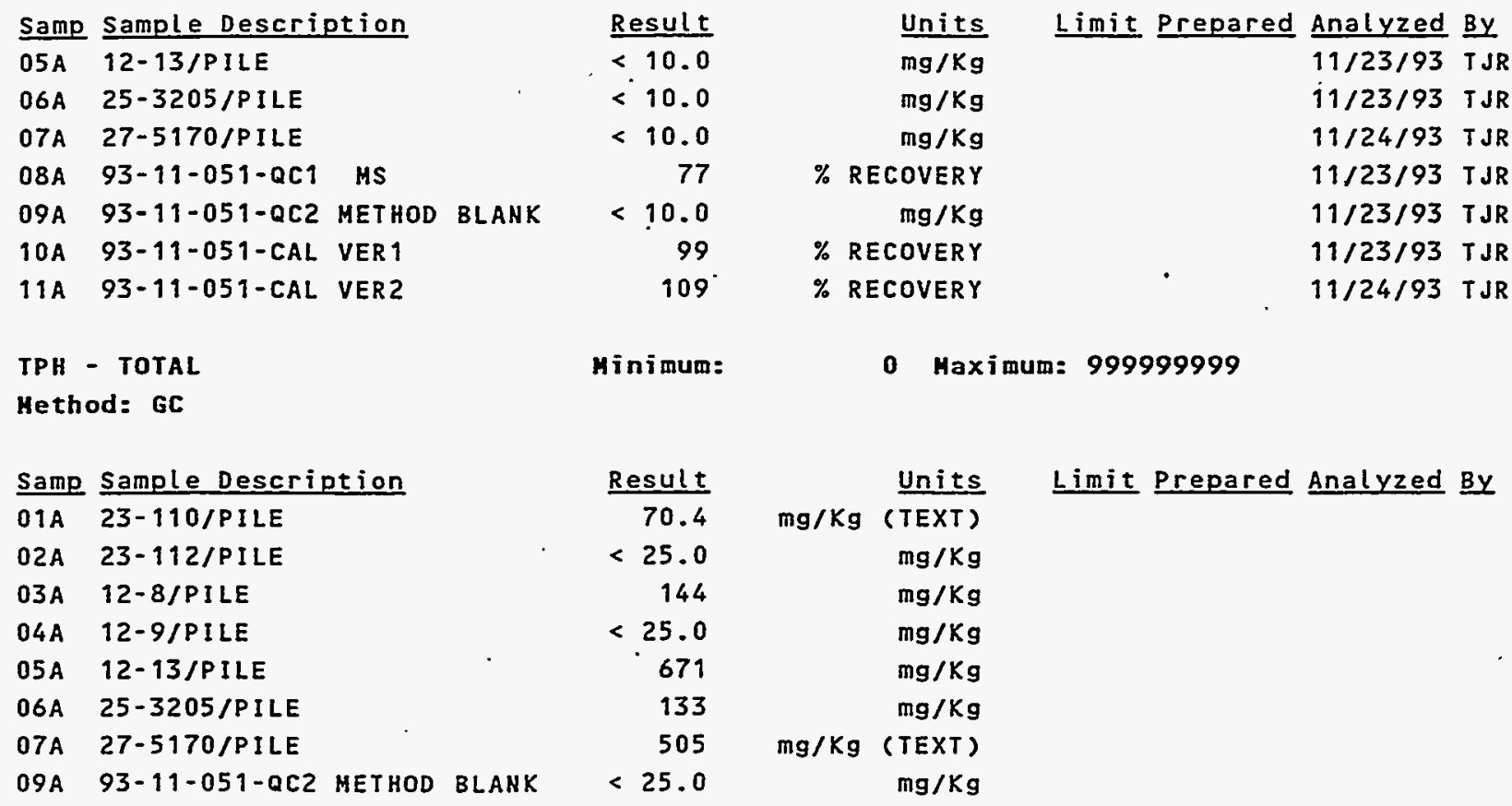


TPH ANALYST'S NOTES:

DIESEL/OIL AMALYSIS OF SAMPLE 23-110/PILE (-01A) PRODUCED A CHROMATOGRAM WHICH EXHIBITS A SLIGHT RESPONSE IN THE ELUTION WINDOW USED FOR OIL QUANTITATION, BUT DOES NOT RESEMBLE THE OIL STANDARDS' CHROMATOGRAPHY. THE COMPONENT'S RESPONSE HAS BEEN QUANTITATED HITH THE OIL CALIBRATION BUT . CANNOT BE CLASSIFIED AS OIL. THE REPORTED RESULT SHOULD BE VIEWED AS A ROUGH CONCENTRATION ESTIMATE OF THE UNKNOWN PRESENT.

THE RESPONSE SEEN IN THE DIESEL/OIL ANALYSIS OF SAMPLE 27-5170/PILE (-07A) FALLS BETHEEN THOSE SEEN WITH THE DIESEL AND OIL STANDARDS. THE OVERALL CHROMATOGRAM SHAPE RESEMBLES THAT OF DIESEL, THEREFORE THE DIESEL CALIBRATIOH WAS USED FOR QUAHTITATING. HOHEVER, THE CONSTITUENT CANHOT BE QUALIFIED AS DIESEL AND THE RESULT SHOULD BE CONSIDERED AN ESTIHATE.

THE OIL MATRIX SPIKE HAS EXTRACTED FROM A PORTION OF -07A. THE CHROMATOGRAPHY AND SPIKE RECOVERY WAS ADVERSELY AFFECTED BY THE ELUTION PROXIMITY OF THE SAMPLE COMPONENT TO THE OIL STANDARD. BOTH THE SAMPLE AND THE OIL MATRIX SPIKE HERE QUANTITATED HITH THE OIL CALIBRATION AND THE DIFFERENCE TAKEN TO DETERHINE THE RECOVERY.

THE DIESEL MATRIX SPIKE WAS EXTRACTED FROM A SAMPLE OF A DIFFERENT WORK ORDER. THE SAHPLE WAS HIGHLY CONTAMINATED WHICH DID NOT ALLOW FOR ADEQUATE RECOVERY OF THE SPIKE. CALIBRATION VERIFICATION STANDARDS HERE ANALYZED HITH THE SAMPLES IN ORDER TO VALIDATE THE HETHODS USED. 





\section{Reynolds Electrical \& Engineering Co., Inc.}

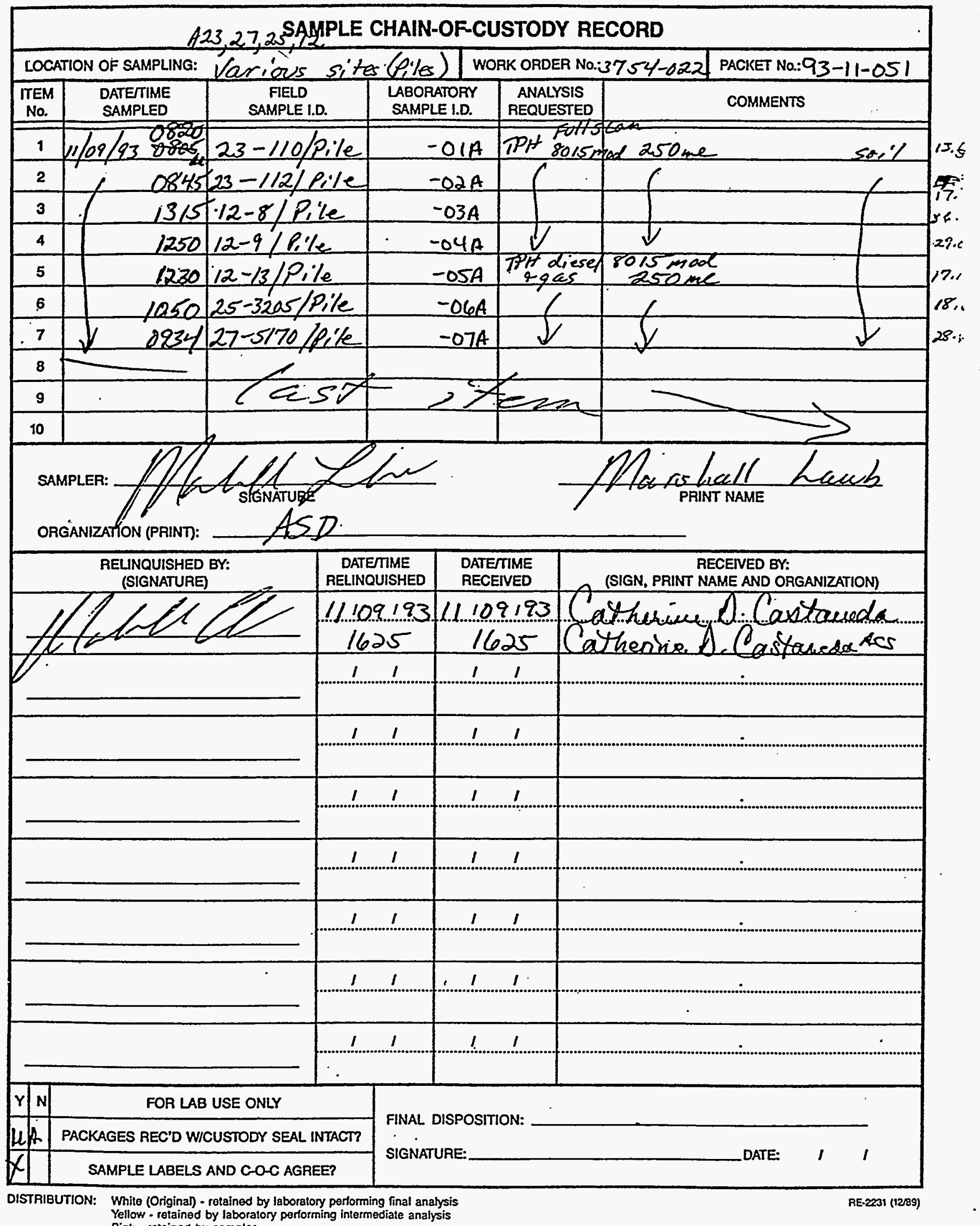




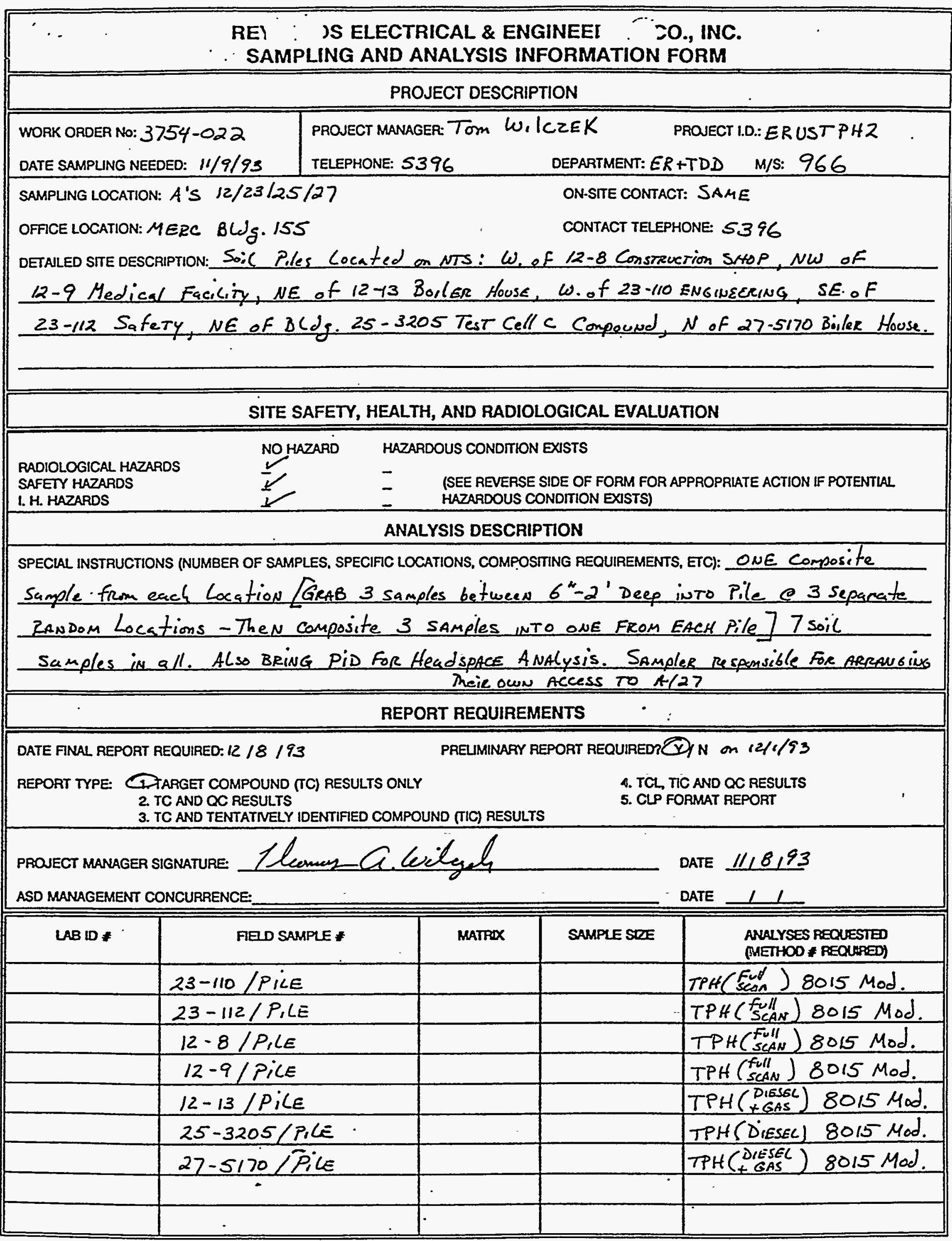


Project No.

Book No.

63

TITLEA23,A27, A25, A12 Riles

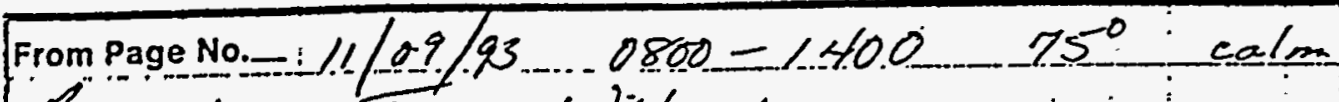

Keguestor-ion Jirkek

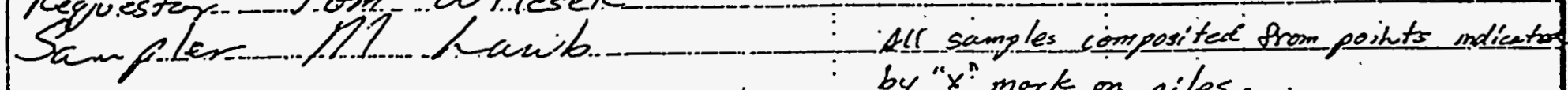

-... Various soil somples of dict pites anosind site. No preservolih

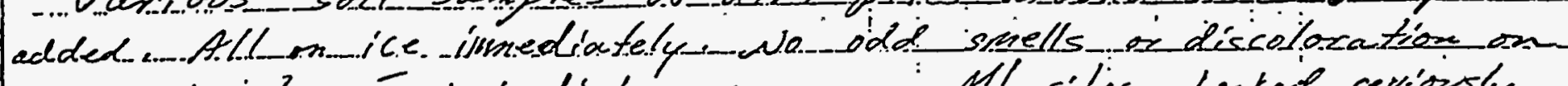
amy samples Typical dist. appearamen : All sites checked reviously by - Radsafe. Points of compesite taken nandomly by me-pess lonew.

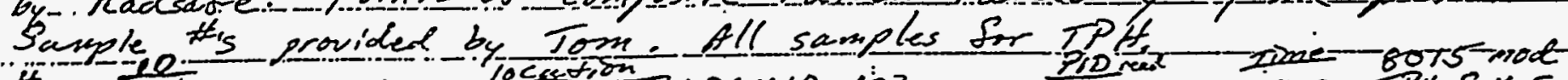

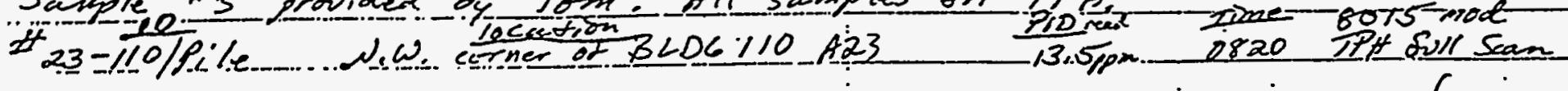

$$
\text { ETh }
$$

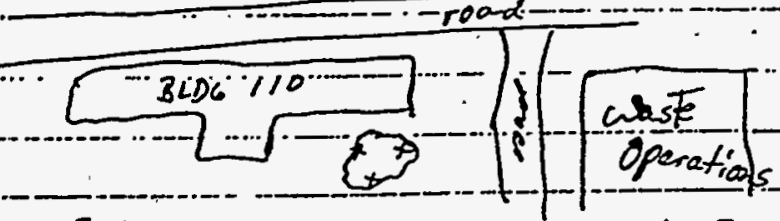

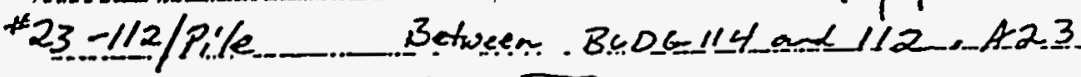

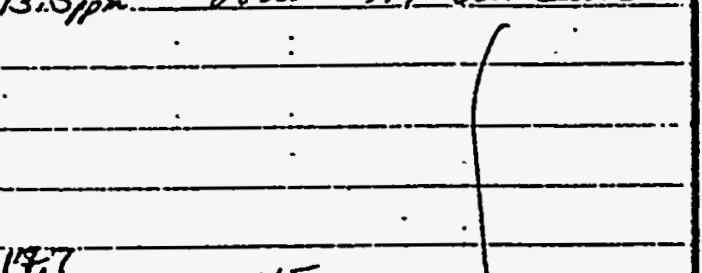

$$
\cdots E=5 .
$$

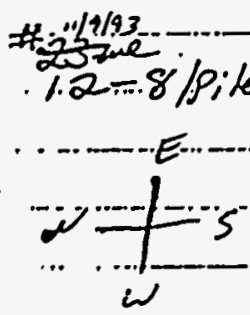

$* 12-q / p i l e$

behind West of medicul 3CDG 9 All<smiles>CC(C)(C)C(C)(C)C</smiles>

Just ...West of BLDG 8. A 12, Fenced off 36,6 - 1315

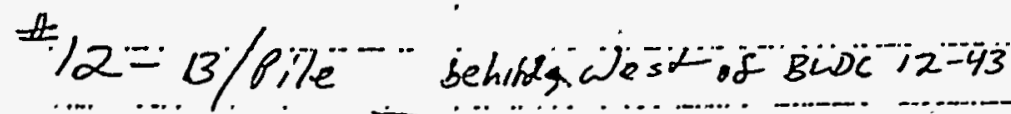

管7

$29.0 \quad 1250$

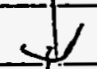

4

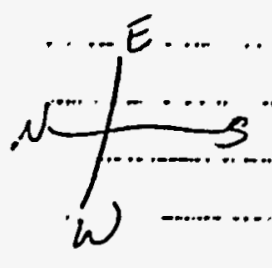

$17 \% 1230$

TPH- $8015 \mathrm{~mol}$

dieselagas

PLDC

(x) - pote

Took- composite -grabs-with ..-Stainhless -Avger-s Approximateh

Witnessed \& Understood by me,

项. $3 f+$ in depth

To Page No.

Date

Date

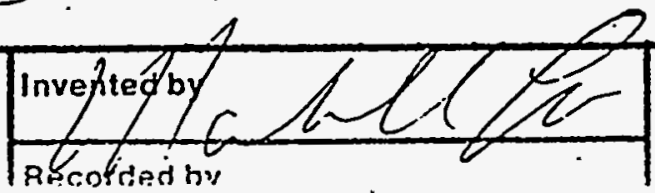

$11 / 08 / 93$ 
64

Book" - TITLE\&25, 227

From Page No.63.

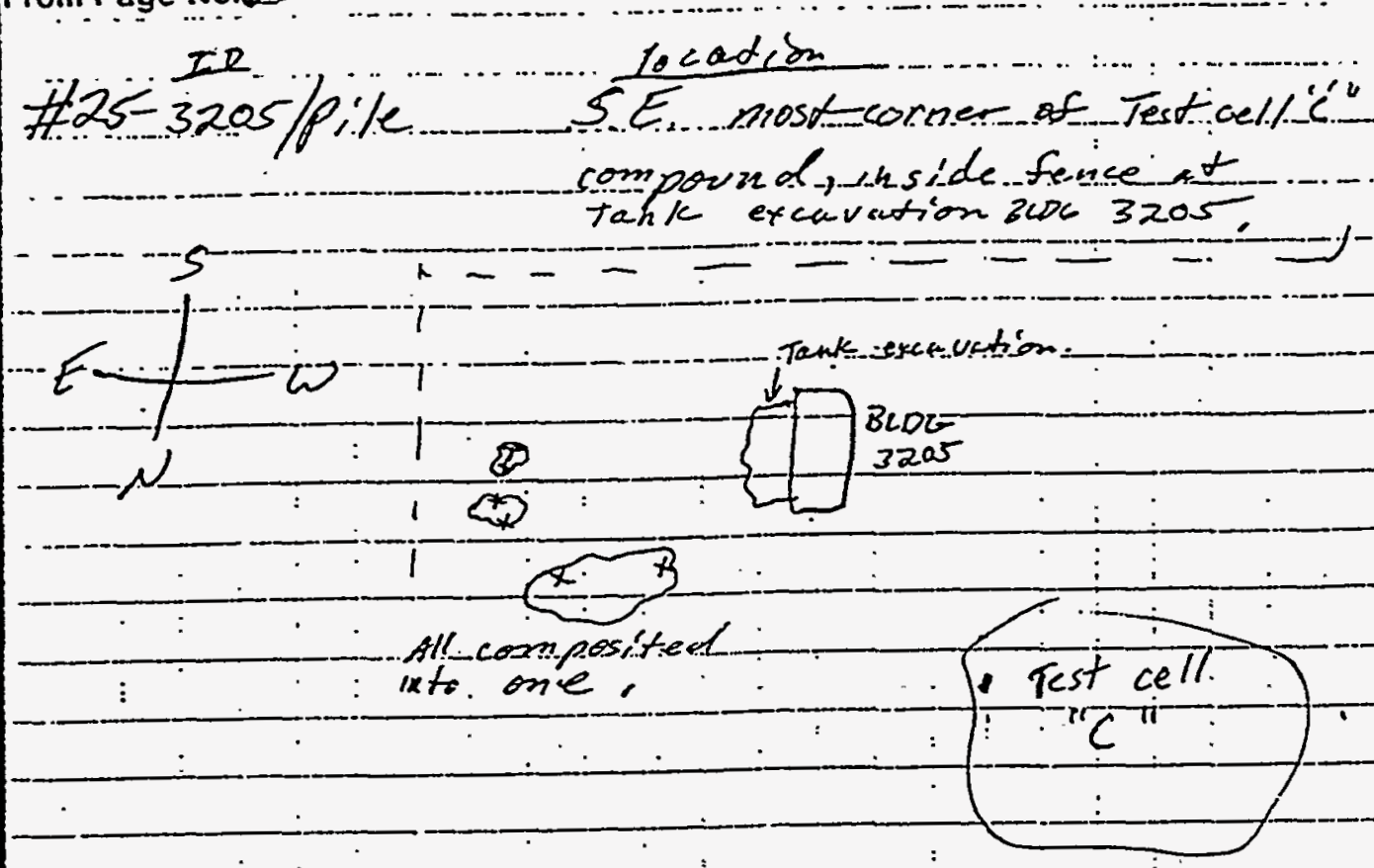

27 - 5170/pile disectly behind (wowth) of $28: 6.0 .934$ BLDG 5170 srem Tank pull

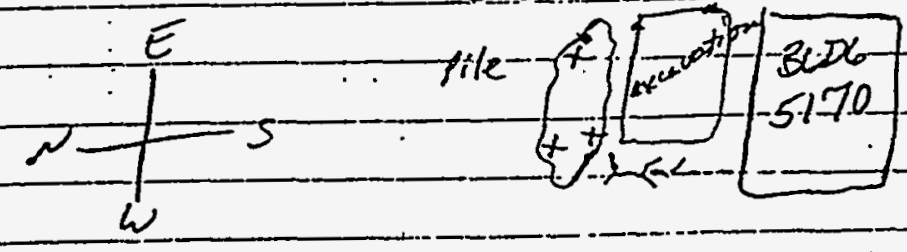
$\frac{\text { Pid } \frac{\text { tike }}{18.6} \cdot \frac{\text { abralysis }}{\text { TPAt.8015 }}}{\text { diesel mox }}$ 
To

S. J. Nacht

From

J. L. Cowley $x$

Date January 12, 1995

Subject SAMPLE ANALYTICAL RESULTS

Enclosed are DATACHEM's results, requested by Jerry Bonn, for the total petroleum hydrocarbons (diesel) analysis of $20 i 1$ samples collected on December 8, 1994, at Dorm B in Area 23.

Please direct any questions you may have to your Client Service Representative, Jerry Dugas, at 295-7220.

$\mathrm{JLC}: 80: \mathrm{ds}$

Enclosure

As Stated

cy $w / 0$ encl.

Central Files, M/S 530

A. R. Latham, M/S 612

ACS Packet No. FY94-12-021

cy $w /$ encl.

J. F. Bonn, ERTD, M/S 969 
REECO

BOB DODGE

ENVIRONMENTAI RESTORATION

TECHNOLOGY DEVELOPMENT

Attn: JERRY BONN

Purchase Order: $3754-038$

Invoice Number:
Order \#: 94-12-021

Date: 01/11/95 15:06

Work ID: TPH(DIESEL ONLY)/SOIL; $\mathrm{A} 23$

Date Received: $12 / 12 / 94$

Date Completed: $12 / 28 / 94$

DCL \#S94-0844

Client Code: ERTD

REPORT TO JERRY BONN, ERTD.

SAMPLES SENT TO DATACHEM FOR ANALYSIS $12 / 13 / 94$.

\section{SAMPLE IDENTIFICATION}

$\begin{array}{lc}\begin{array}{l}\text { Sample } \\ \text { Number }\end{array} & \begin{array}{c}\text { Sample } \\ \text { Description }\end{array} \\ 01 & \frac{12}{12-13-1 / \mathrm{E}} \\ 12-13-1 / \mathrm{SP} 1\end{array}$

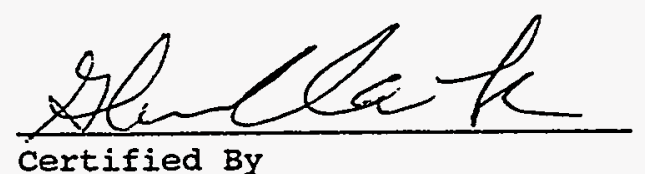






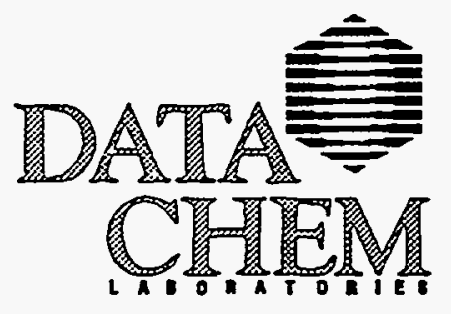

\section{Case Narrative \\ DCL Set ID \#(s): S94-0844-CC}

Client: REECO

Packet \# 94-12-021

Account \#: 3018

23 December, 1994

$94-12-021$

Analysis Method \#: SW8015 modified.

General Set Information: None.

Sample Preparation: 4 hour shake.

Continuing Calibration Verification: Met QC criteria.

Reagent Blank Analysis: Met QC criteria.

Method Blank Analysis: Met QC criteria.

Laboratory Control Sample Analysis: Met QC criteria.

MS/MSD Analysis: Met QC criteria.

. Data Flagging Codes: None were used.

Miscellaneous Comments: None

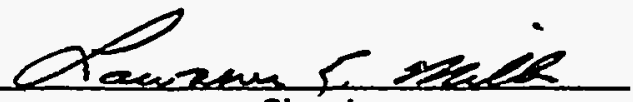

Signature

This report is 7 total pages. 


\section{DATA CHEM \\ $A$ Sorenson Company}

REECO

P. O. Box $98521, \mathrm{M} / \mathrm{S} 713$

Las Vegas, NV 89193-8521

Attention: Jerry Dugas

ANALYTICAL REPOORT

Form ARF-AL

Page 1 of 1

Part 1 of 1

Date

\section{DEC 271994}

Agency Identification Number $\$ 94-0844-C C$

Account No. 03018

$$
94-12-021
$$

FAX (702) 295-7534 Telephone (702) 295-7997

Sampling Collection and Shipment

Sampling Site A23, Dorm B

Date of Collection December 08; 1994

Date Samples Received at Laboratory December 14, 1994

Analysis

Method of Analysis EPA 8015MOD

Date(s) of Analysis December 21, 1994

Analytical Results

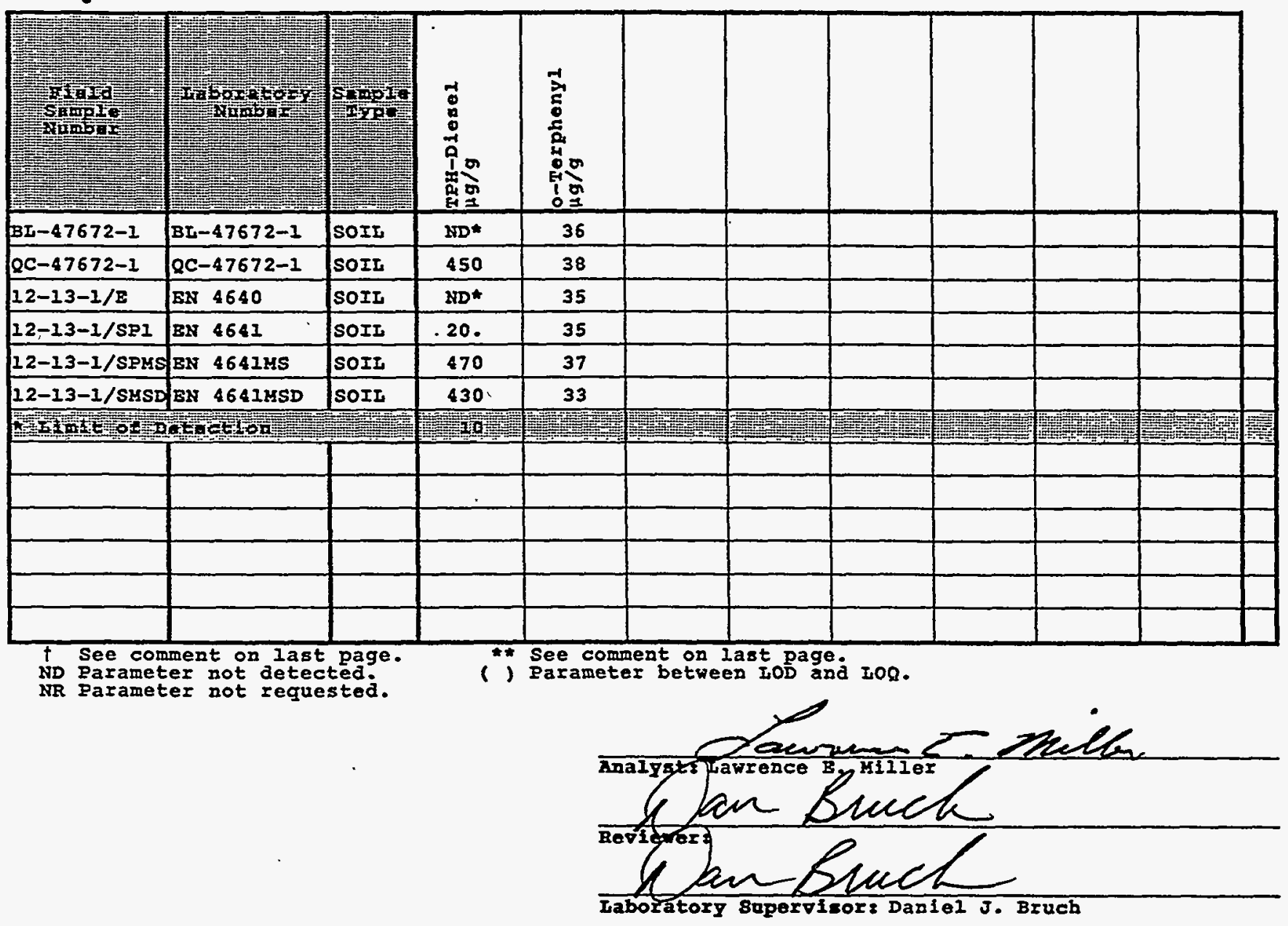

960 West LeVoy Drive / Salt Lake City, Jtah 84123-2547 / (801) 266-7700 002 A Sorenson Company 


\section{DATA CHEM S Sorenson Company}

Client Hame......... REECO

Release Number....... Not Provided

Matrix.............. soIr

Reporting Un1ts..... $\mathrm{Hg} / \mathrm{g}$

Report Bas1z........ [... X]s Received $\square$ Dried
FORM G (TYPE I)

SINGLE METHOD ANALYSES

\section{QUALITY CONTROL DATA SHEET} SURROGATE SUMMARY
Form RLIMS63G-V1.0

PDOR 12229415165107

Page 6

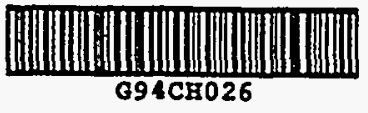

Report Date...... 12/22/94 $15: 16$

DCL Analysis Group: 694CH026

Analysis Method...2 BPA 8015HOD

DCL Prep Group..... 094CH026

Preparation Mothods

Surrogate Recoveries

\begin{tabular}{|c|c|c|c|c|c|c|c|c|c|}
\hline $\begin{array}{l}\text { Surr. ID } \\
\text { QC IImIts }\end{array}$ & \multicolumn{3}{|c|}{$\frac{0-\text { Terphenpl }}{50.0 / 125 .}$} & & & & & & \\
\hline $\begin{array}{l}\text { DCL samplo } \\
\text { Number }\end{array}$ & $\begin{array}{l}\text { Analyze } \\
\text { Result }\end{array}$ & $\begin{array}{l}\text { Spikeo } \\
\text { Amount }\end{array}$ & Rec. & $\begin{array}{l}\text { Analyte } \\
\text { Result }\end{array}$ & $\begin{array}{l}\text { Splked } \\
\text { Amount }\end{array}$ & Rec. & $\begin{array}{l}\text { Analyze } \\
\text { Result }\end{array}$ & $\begin{array}{l}\text { Spiked } \\
\text { Amount }\end{array}$ & Rec. \\
\hline$B E-47672-1$ & 35.9 & 40.0 & 89.8 & & & & & & \\
\hline EN 4640 & 34.9 & 40.0 & 87.3 & & & & & & \\
\hline EN 4641 & 34.5 & 40.0 & 86.3 & & & & & & \\
\hline EN $4641 \mathrm{MS}$ & 37.2 & 40.0 & 92.9 & & & & & & \\
\hline EN 4641MSD & 32.9 & 40.0 & 82.4 & & & & & & \\
\hline$g c-47672-1$ & 38.2 & 40.0 & 95.6 & - & & & & & \\
\hline
\end{tabular}

960 West LeVoy Drive / Salt Lake City, Utah 84123-2547 / (801) 266-7700003 FAX (801) 268-9992 


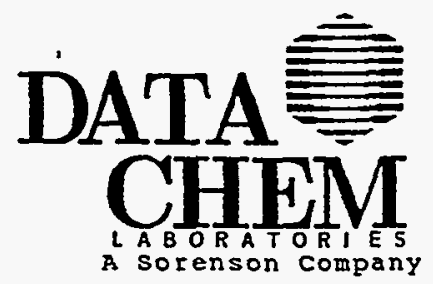

Client Name......... REECo

Release number...... Not Provided

Matrix............ SOIL

Reporting units.....: $\mu g / g$

Report Basis....... $囚$ As Received $\square$ Dried

DCL Preparation Group: G94CH026

Date Prepared.......: $12 / 16 / 94 \quad 00: 00$

Preparation Method...:

Analytical Results

\section{FORM C (TYPE 1) SINGLE METHOD ANALYSES \\ QUALITY CONTROL DATA SHEET \\ BLANK SAMPLE}

Form RLIMS63C-V1.0

PDOR 12229414213422 Page 9

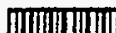

$594 \mathrm{CHOOL}$

Report Date.....: 12/22/94 14:21

DCL Sample Name...: BL-47672-1

DCI Analysis Group: $694 \mathrm{CH} 026$

Analysis Method...: EPA 8015 MOD

Instrument Type...: GC

Instrument ID.....:

Column Type...... Not Applicable

\begin{tabular}{|l|c|c|c|}
\hline Analyte & Date & Result & CRDL \\
\hline TPH-Diesel & $12 / 21 / 9417: 33$ & 2.27 & 10 \\
\hline
\end{tabular}




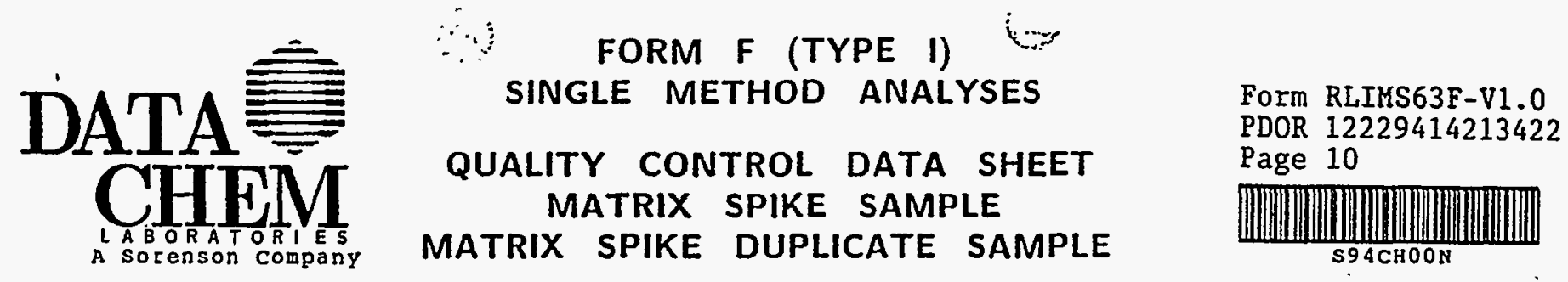

Client Name.......... REECo

Release Number....... Not Provided

Matrix............. sorr

Reporting units...... $\mu g / g$

Report Basis........ 因As Received $\square$ Dried

Report Date......: 12/22/94 14:21

DCI sample Nare...: EN 4641MS

DCL Analys is Group: $694 \mathrm{CH} 026$

Analysis Method...: EPA $8015 \mathrm{MOD}$

Instrument rype...: GC

Instrument ID.....:

Column Type....... Not Applicable

DCL Preparation Group: G94CH026

Date prepared........: $12 / 16 / 9400: 00$

Preparation Method...:

Analytical Results

\begin{tabular}{|c|c|c|c|c|c|c|c|}
\hline Analyce & $\begin{array}{c}\text { Date } \\
\text { Analyzed }\end{array}$ & $\begin{array}{l}\text { Sample } \\
\text { Result }\end{array}$ & $\begin{array}{l}\text { Spiked } \\
\text { Result }\end{array}$ & $\begin{array}{l}\text { Spike } \\
\text { Added }\end{array}$ & $\begin{array}{l}\text { Percent } \\
\text { Recovery }\end{array}$ & Iimits & $\begin{array}{l}\text { Qc } \\
\text { Flag }\end{array}$ \\
\hline TPH-Diesel & $12 / 21 / 9420: 02$ & 19.5 & 472. & 500 & 90.5 & $50.0 / 125$ & \\
\hline
\end{tabular}

Analytical Results

\begin{tabular}{|c|c|c|c|c|c|c|c|c|}
\hline Analyte & $\begin{array}{c}\text { Date } \\
\text { Analyzed }\end{array}$ & $\begin{array}{c}\text { Duplicate } \\
\text { Result }\end{array}$ & $\begin{array}{l}\text { Percent } \\
\text { Recovery }\end{array}$ & Mean & Range & RPD & $\begin{array}{c}\mathrm{QC} \\
\text { Iimits }\end{array}$ & Flag \\
\hline TPH-Diesel & $12 / 21 / .94 \quad 20: 52$ & 435. & 83.0 & 453. & 37.2 & 8.6 & $0.000 / 50.0$ & \\
\hline
\end{tabular}




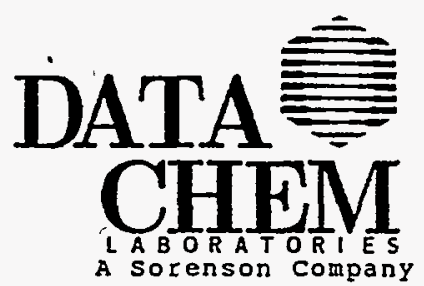

Client Name......... REECo

Release Number...... Not Provided

Matrix................ sord

Reporting units...... $\mu g / g$

Report Basis........ 囚As Received $\square$ Dried

LCs source..........

DCL Preparation Group: $694 \mathrm{CH} 026$

Date Prepared........: 12/16/94 00:00

Preparation Method....
FORM B (TYPE 1) SINGLE METHOD ANALYSES

QUALITY CONTROL DATA SHEET

LABORATORY CONTROL SAMPLE (LCS)
Form RLIMS63B-V1.0 PDOR 12229414213422 Page 11

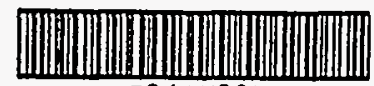

S94CHOOM
Report Date......: 12/22/94 14:21

DCL Sample Name...: QC $-47672-1$

DCL Analysis Group: $694 \mathrm{CH} 026$

Analys is Method...: EPA 8015 MOD

Instrument Type...: GC

Instrument ID.....

Column Type....... Not Applicable

Analytical Results

\begin{tabular}{|c|c|c|c|c|c|c|}
\hline Analyte & $\begin{array}{c}\text { Date } \\
\text { Analyzed }\end{array}$ & Target & Result & $\begin{array}{l}\text { Percent } \\
\text { Recovery }\end{array}$ & $\begin{array}{l}\text { QC } \\
\text { Limits }\end{array}$ & $\begin{array}{l}\text { QC } \\
\text { Flag }\end{array}$ \\
\hline PPH-Diesel & $12 / 21 / 94 \quad 16: 44$ & 500 . & 450. & 90.0 & $50.0 / 225$. & \\
\hline
\end{tabular}

960 West LeVoy Drive / Salt Lake City, Utah 84123-2547/(801) 266-7700006 FAX (801) 268-9992 
SUPPORTING DATA 



\section{DATA PACKAGE REVIEW}

ACS PACKET NUMBER: $94-12-021$

LABORATORY:

DATACHEM

DATE REVIEWED:

$12-28-94$
CLIENT: ERTD - J. BONN

REVIEWED

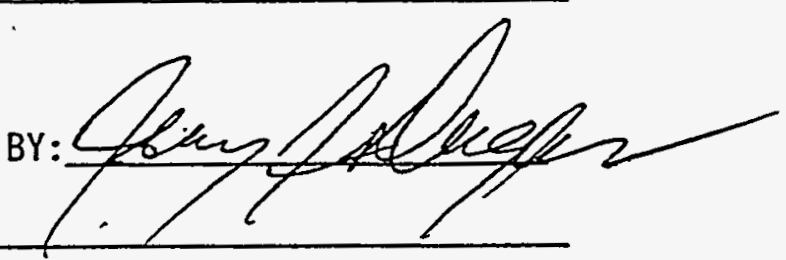

TPH (DIESEL)

MATRIX:

SOIL

ARE TPH DATA ACCEPTABLE:

YES

\section{ARE CRITERIA MET}

1. QC SAMPLES ACCEPTABLE

2. MATRIX SPIKE ACCEPTABLE

3. METHOD BLANK ACCEPTABLE

4. HOLDING TIMES ACCEPTABLE

\begin{tabular}{c} 
YES \\
\hline YES \\
\hline YES \\
\hline YES
\end{tabular}

a. Date Sampled:

b. Date ACS Received:

c. Date Contract Lab Received:

d. Date Extracted:

e. Date Analyzed:

$\frac{\frac{12-8-94}{12-12-94}}{\frac{12-14-94}{12-21-94}} \frac{\text { YES }}{\frac{\text { YES }}{\text { YES }}}$

\# DAYS $\quad$ ACCEPTABLE

5. CALCULATIONS ACCEPTABLE:

6. Correct conc. units used:

7. FORMS ACCEPTABLE:

8. Chain-of-Custody Agrees:

$\frac{0}{4}$

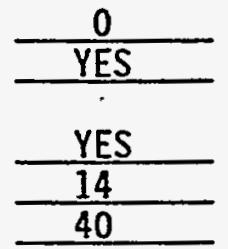

COMMENTS:

EFRE-2261 (03/93) 


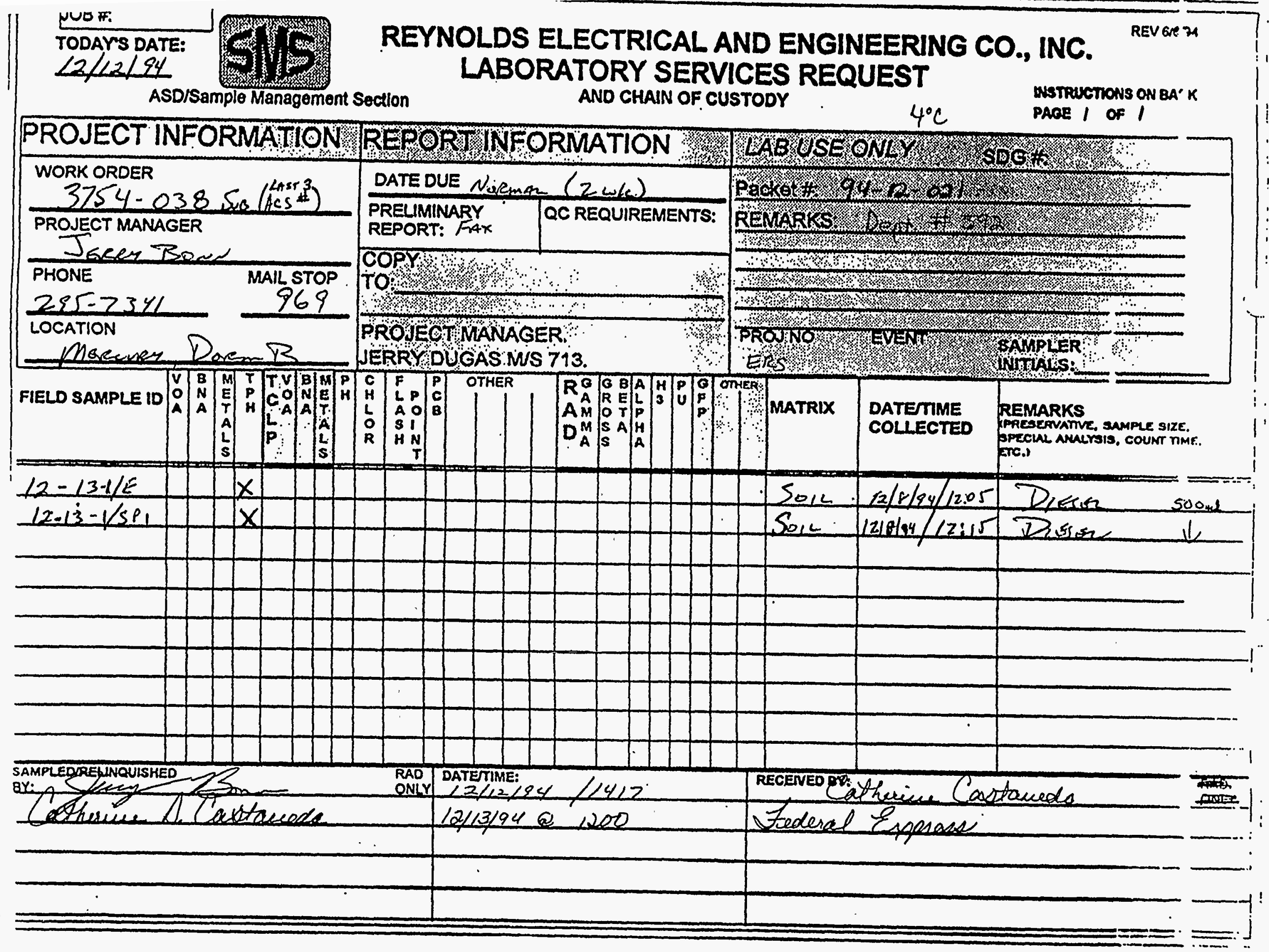


PACKET N0: $94-12-021$

\section{DATE RECEIVED: $12 / 12 / 94$}

BY: cic

1. DO SAMPLE LABELS AGREE WITH CHAIN-OF-CUSTODY? IF NOT, LIST DISCREPANCIES NOTED:

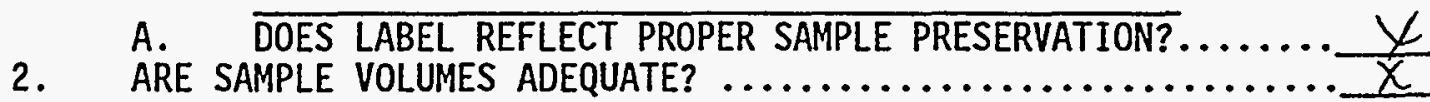
IF NOT, LIST DISCREPANCIES NOTED:

3. ARE SAMPLES FROM A KNOWN OR SUSPECTED RAD EXCLUSION AREA.... A. HAS A SMS RADIOLOGICAL SAMPLE SURVEY BEEN PERFORMED... $x$

B. IS SAMPLE $<1000 \mathrm{dpm} / 100 \mathrm{~cm}_{2}$ or $450 \mathrm{pCi} / \mathrm{mL}$ ? ........... IF NOT, HAS SAMPLE BEEN SENT TO RC LAB FOR RESULTS?...

4. CHAIN-OF-CUSTODY
A. SAMPLES RECEIVED IN GOOD CONDITION/PROPERLY COLLECTED/ PRESERVED (GLASS FOR ORGANICS/PLASTIC FOR METALS) ...... $x$ 1) IS THERE ZERO HEADSPACE FOR VOA SAMPLES........... IF NOT, LIST DISCREPANCIES NOTED:

B. SAMPLE CUSTODY SEALS (IF ANY) ARE INTACT ...............

C. IS COOLER TEMPERATURE RECORDED ON CHAIN-OF-CUSTODY?

D. HAS WORK ORDER \# BEEN PROVIDED?

E. HAS A SAMPLING LOCATION BEEN PROVIDED?

F. HAS THE DATE AND TIME SAMPLED BEEN PROVIDED?

G. IS THE C-0-C SIGNED, DATED AND TIME ENTERED BY PERSON TURNING IN THE SAMPLES?...................... $X$

5. ASD - Sample Collection Sheet or IH Sample Data Sheet

A. IS THE SAMPLE/COLLECTION/DATA SHEET PROVIDED?...

B. IS THE FOLLOWING INFORMATION PROVIDED?

1. WORK ORDER NUMBER.

2. SAMPLING LOCATION...

3. IS SAMPLE FROM RAD EXCLUSION AREA IS RAD ANALYSIS REQUESTED? IF YES, DOES REQUEST SHOW ASD AS CONTACT

4. IS TURN-AROUND TIME OR DUE DATE PROVIDED?..

5. DOES CHAIN-OF-CUSTODY AND SAMPLE DATA SHEET AGREE ON CUSTOMER REQUESTED ANALYSES
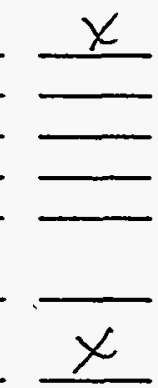

$\mu$

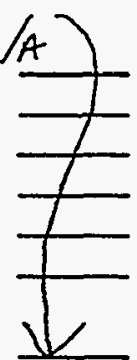

6. FOR IH ANALYSES: HAS AIR VOLUME BEEN PROVIDED? $\ldots \ldots \ldots \ldots \ldots, \mu$

7. FOR BLOOD-LEAD: ARE NAMES AND SSNS PROVIDED?.............

8. FOR METALS: IS SAMPLE PRESERVED W/HNO3 TO $\mathrm{pH}<2$ ? $\ldots \ldots \ldots \ldots \ldots$ 


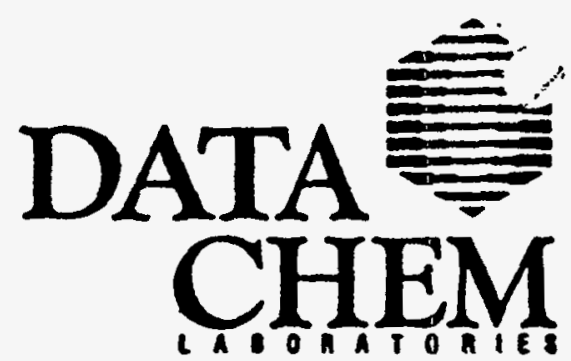

\section{ANALYTICA REQUEST FORM}

\section{Deqular status}

\section{RUSH Stalus Requeated - ADDITONAL CHARGE}

RESULTS REQUIRED BY

CONTACT DATACHEM LABS PRIOR TO SENDING SAMPLES.

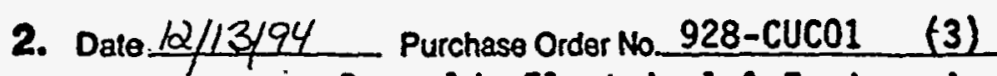

3. Company Name Reynolds Electrical \& Engineering Co.

Address P. O. Box 98521 , M/S 713

Las vegas, NV 89193-8521

Person to Contact Jerry Dugas

Telephone ( 702) 295-7997

Fax Telephone( 702)_295-7534

Billing Address (if different from above)

Contracts Administration H/S 692

Las Vegas, NV 89193-8521
4. Quote No.

DCL Project Manager

5. Sample Collection

Sampling Sito A23, Dorm B

Industrial Procoss

Dale of Collection $12 / 08 / 94$

Time Collected 12:05

Date of Shipment 12/13/94

Chain of Custody No. $94-12-021$

6. REQUEST FOR ANALYSES

\begin{tabular}{|c|c|c|c|c|}
\hline $\begin{array}{l}\text { Laborabory } \\
\text { Use Onty }\end{array}$ & $\begin{array}{l}\text { Cliond Samplo } \\
\text { Number }\end{array}$ & $\begin{array}{l}\text { Modis } \\
\text { Typo" }\end{array}$ & $\begin{array}{c}\text { Sample Volume } \\
\text { (Liters) }\end{array}$ & ANALYSES REQUESTEO - Uso Hethod Number i Knom \\
\hline & $12-13-11 E$ & Soil & $500 \mathrm{ml}$ & TPH (Diesel Ouly) $\quad 8015 \mathrm{~m}$ \\
\hline & $12-13-1 / 58$ & $\mathrm{~V}$ & $\sqrt{2}$ & 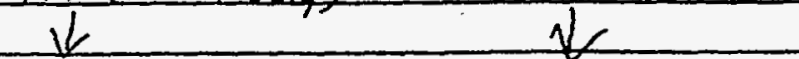 \\
\hline & & & & \\
\hline & & & & \\
\hline & & & & \\
\hline & & & & . \\
\hline & & & & \\
\hline & & & & \\
\hline & & & & \\
\hline & & & & \\
\hline & & & & \\
\hline & & 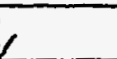 & & \\
\hline
\end{tabular}

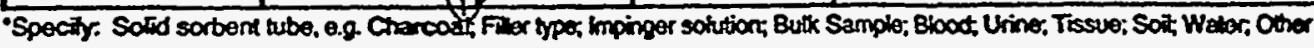
6. O C REQUIREMENTS MUST BE COMPLETED FOR ENVIRONHENTAL SAMPLES - SEO
General Services Terms and
- Conditions: QC samples bated
at regular sample rate
$\square$ METHODQC SAMPLES Rab $\propto C$ according to published methods]
PROJECT PLAN OC SAMPLES
Rab $Q C$ according to provided OANQC Plan)
DOQC SAMPLES REQUESTED
(May not conform to Agency requirements)

$\square$ OTHER (as specified below)

Comments Please provide: All raw data; invoice must be submitted with report. Reference

Packet: $94-13-021$ on narrative and invoice. Please note that this information is a requirement for all work submitted from REECO. Per agreement with Rand Potter.

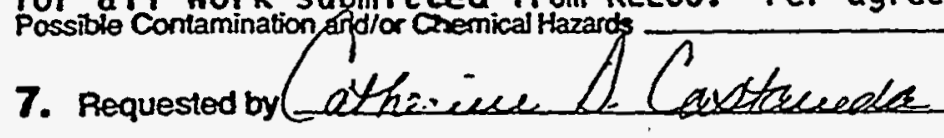

960 West LeYoy Ditue / Salt Lake City, UT 84123 4388 Glendal-Mllford Basd / Cincinnatl, OH 45242

800-356-9135 or 80t-266-7700 I FNX: 801-268-9992 800-458-1493 or 513-733-5336 / FAX: 513-733-5347

DATACHEM LABORATORES - A SORENSON COMPANY

DISTRIBUTRON:

WHITE - LABORATORY COPY

CANARY - CUSTOMER COPY 


\section{APPENDIX C}

\section{WASTE MANIFESTS}





\title{
Rey...Jlds Electrical \& Engineering Co., unc.
}

\section{NTS ONSITE WASTE TRANSPORT' MANIFEST}

1. GENERATOR'S NAME, ORĞANIZATION, AND LOCATION (IF REECO, INCLUDE DEPT. NO.) (PLEASE PRINT):

\author{
Abandoned Site, U.S.T. \\ Site 12-13-1 \\ Reea 12, Boiler House, NTS
}

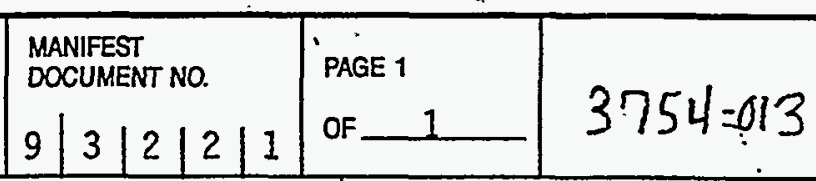

$3754=013$

GENERATOR'S PHONE( )

\section{3a. TRANSPORTER NAME (PLEASE PRINT) .}

Direct to: Rollins Custom Environmental Transport

4. U.S. DOT DESCRIPTION (INCLUDE PROPER SHIPPING NAME, HAZARD CLASS AND ID NUIMBER, EPA WASTE CODE, AND WASTE STREAM AND PACKAGE IDENTIFICATION NUMBERS)

\begin{tabular}{l|l|l}
\hline & HM & RQ, Waste Combustible Liquid, n.o.s.
\end{tabular}

A. Combustible Liquid, NA 1993, (Diesel Fuel w/

. XX $\begin{aligned} & \text { Metals, D008,D009, F002, F003, F005) } \\ & \text { 12-ABDN-042-AM-93-001 }\end{aligned}$

B.

\begin{tabular}{ll|l}
\hline C. & & \\
\hline
\end{tabular}

\begin{tabular}{|l|l|}
\hline D. & \\
\hline
\end{tabular}

USE CONTINUATION PAGES FOR ADOITIONAL ITEMS AS NECESSARY

8. SPECIAL HANDLNG INSTRUCTIONS AND ADDTIONAL INFORMATION:

a) 93778

Máterials directly transferred to Rollins for transhipment to OPC at Los Angeles, CA. ; $* * * * * * * *$ FOR TRACKING PURPOSES ONLY********

9. GENERATOR'S CERTIFICATION:

I HEREBY CERTIFY THAT THE CONTENTS OF THIS CONSIGNMENT ARE FUUY AND ACCURATELY DESCRIBED ABOVE BY PROPER SHIPPING NAME AND ARE CLASSIFIED, PACKED, MARKED, AND LABELED, AND ARE IN ALL RESPECTS IN PROPER CONDITION FOR HIGHWAY TRANSPORT ACCORDING TO APPLICABLE REGULATIONS.

I FURTHER CERTIFY THAT THE CONTENTS INFORMATION ABOVE IS TRUE AND CORRECT TO THE BEST OF MY KNOWLEDGE THROUGH PROCESS KNOWLEDGE OR LABORATORY ANALYSIS. I CERTIFY THAT, WHERE APPLICABLE, I HAVE APPROPRIATELY SEGREGATED WASTES AND HAVE MADE A GOOD-FATTH EFFORT TO MINIMIZE THE AMOUNT OF WASTE GENERATED AT WORK LOCATIONS UNDER MY PURVIEW.

10. GENERATOR'S SIGNATURE:

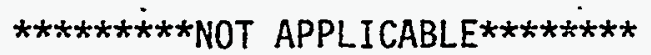

11. TRANSPORTER'S SIGNATURE

$\star \star \star * * * * * * *$ NOT APPLICABLE********

12. DISCREPANCY INDICATION:

13. DISPOSALACCUMULATION SIJE SIGMHJ6RE (ACKNOWLEDGES ACCEPTANCE OF WASTE)

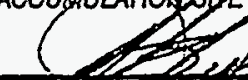

Q $27-93$

DISTRIBUTION: White-DWMD Area 5 Canary - Genẹtator? 
-

$-\ldots$ 
APPENDIX D

FIELD NOTES 



\section{UST FIELD CONFIRMATION}

(Provide sketch of surface features on back of sheet)

DATE: $9 / 16 / 92$ (3:00 pm)

ECO PERSONNEL: TAW

LOCATION (NEAREST LANDMARK): No of BdLg. 12-13. By Area 12 Cafeferia

Parking LoT. Area 12.

CONTENTS: Deisel + $H_{2} \mathrm{O}$ (TWO PhASE LiQUiD)

STATUS (ACTIVE, INACTIVE): UNK (PROBABLE INACTIVE)

VENT PIPES: ONE - Found on $\omega$. SIDE of $3 d C_{g} 12-13$

FILL PIPES: ONE -IMBEDDED IN $30^{\prime \prime} \times 30^{\prime \prime}$ CONCRETE PAD

DEPTH TO BOTTOM OF TANK: APPROX $9{ }^{\circ}$ " FROM B̈TTOM of TANK TO TOP of Fill TUBE DEPTH TO TOP OF TANK: PROduct in BOTTOM 28" of TANK. (Approx 3 "of $\mathrm{H}_{2} \mathrm{O} / 25^{\prime}$ of Deisel) ORIENTATION: UNK (PQSS. E-W)

CAPACITY: UNK

CONSTRUCTION MATERIAL (TANK): METAC

LEAK DETECTION EQUIPMENT: NONE

PIPING MATERIAL: NONE

PIPING DESTINATION: UNK

FACILITY OPERATOR : UNK

COMMENTS: No Gayse Found fo Near mank To estinate the volume of Product,
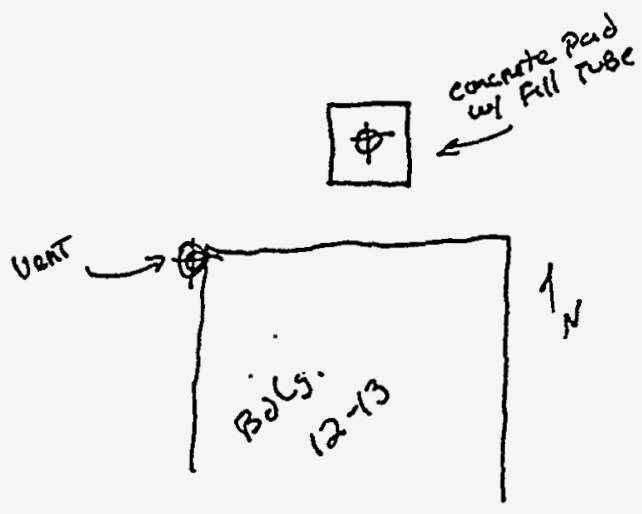
$9 / 16 / 92$ i

- Arimed o area 120 3.00 m. tand tuh fraily ecielly Proveded to tent fre presene fo lyenid w inpsteh. Siquid froud: isotw $28^{\circ}$ of tuh. Use Kalcurata sampler to bring up pordect. Niscaneres it wes a two -plase liquid mixture of Deisel $+\mathrm{H}_{2} \mathrm{O}$. Requeted Suplen cite two full set \& sumples, ore ead for deisel + cuettor

for water. Semples fran tenh 12-13 ines olituriel $23: 30 \mathrm{pm}$. They were lablel It is estrivilad that 3 "of liquid is vater. and the rencing 25 " of tyenel is chesel Sauples were loysed ond coild accorndigh laih wow recapped. Left site e 4:00 pm. 
Sample ho cations: Area 36 Bldg. 2105; Aves 11 Tweeze

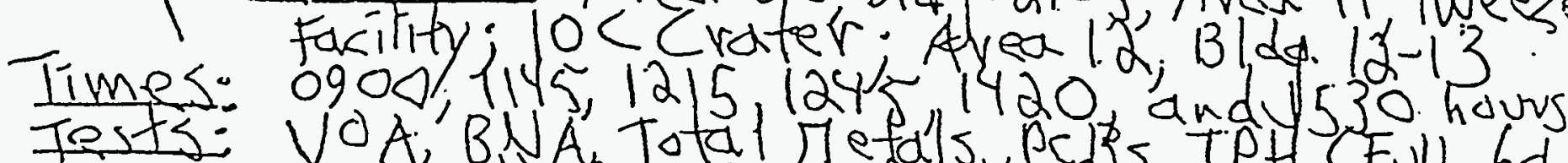

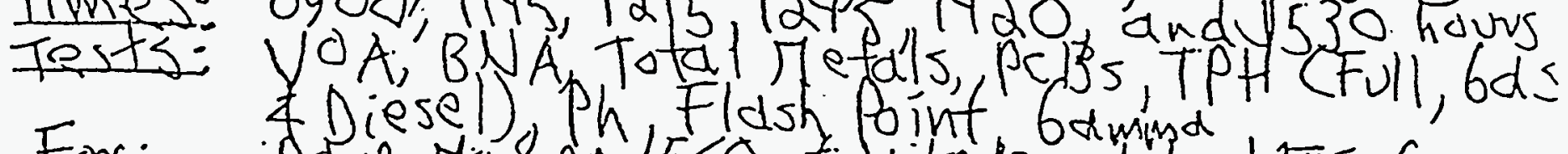

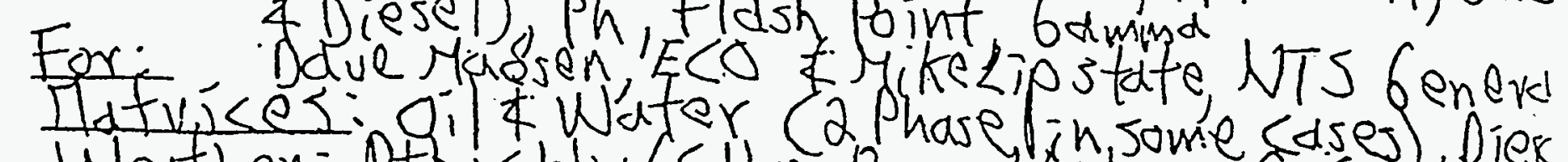

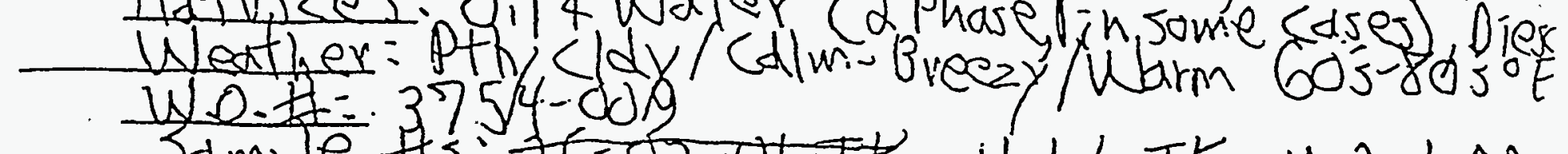

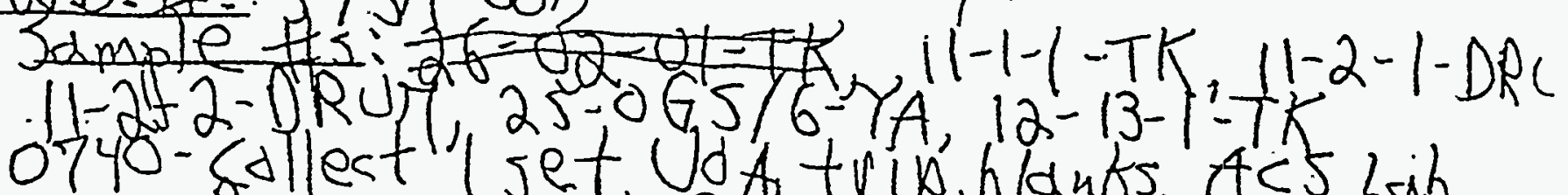
0740- Select 1 set Udo A trip blanks AC5 Lab

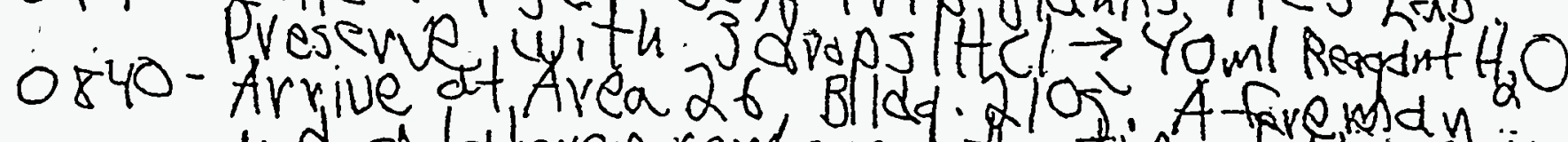

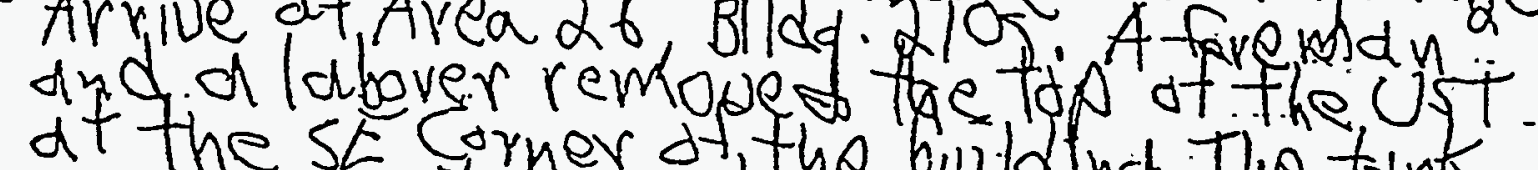
was completerner at the build ind The tank tom wi le e le of 1000 - accompany me try the day hat site a took

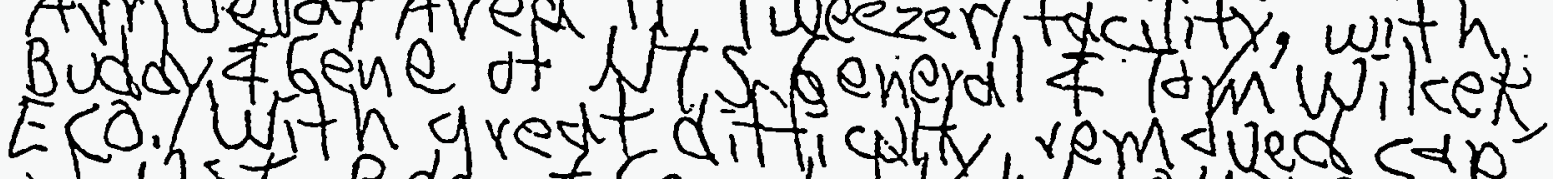

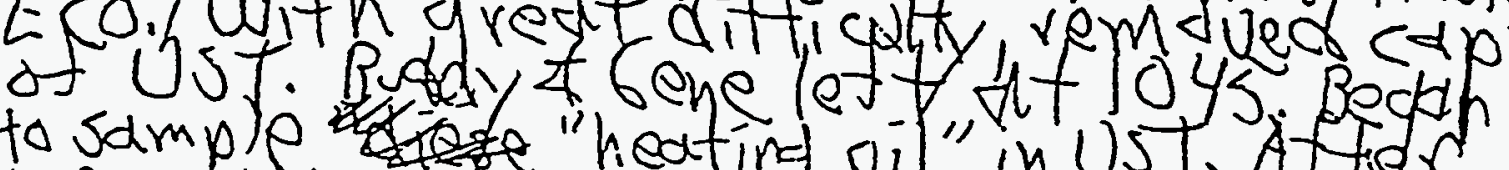

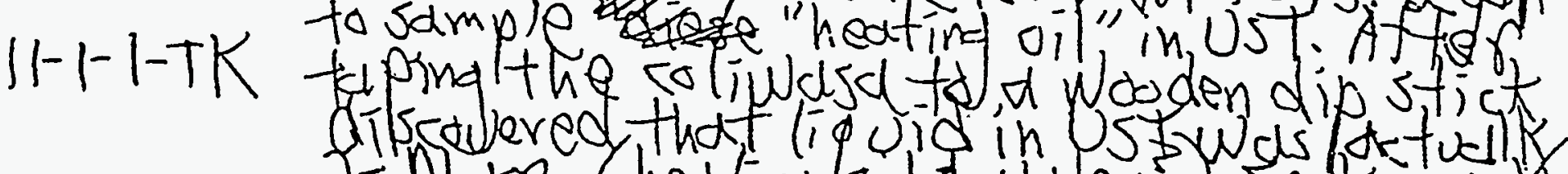

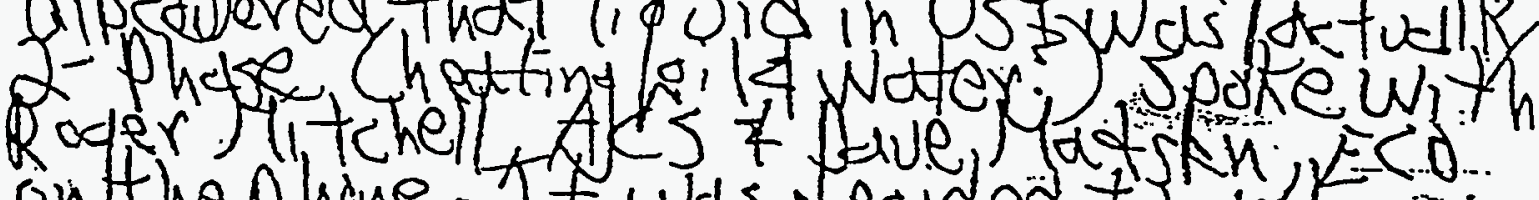

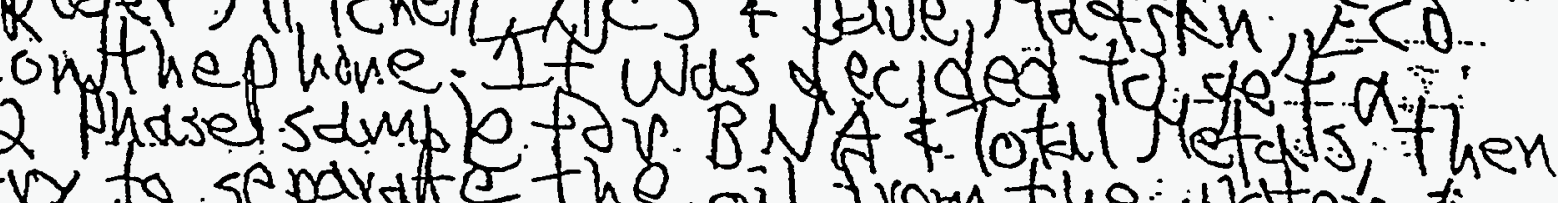
try to separate the oil from the wader This was done and washery time consuming

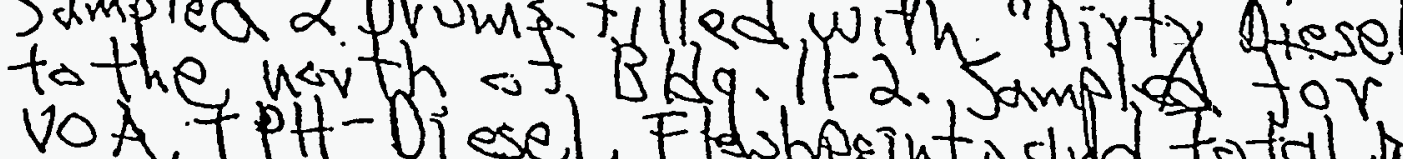

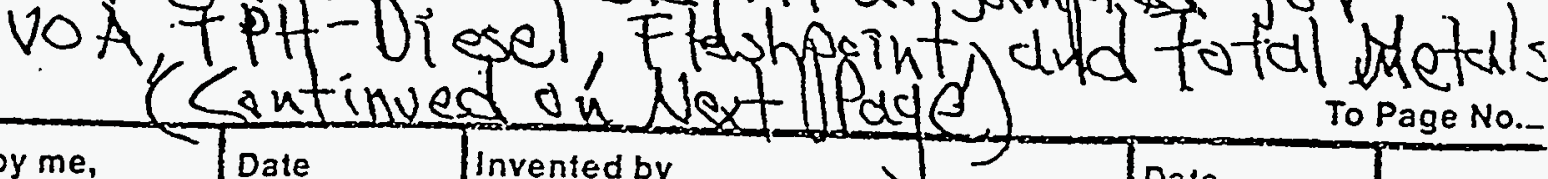




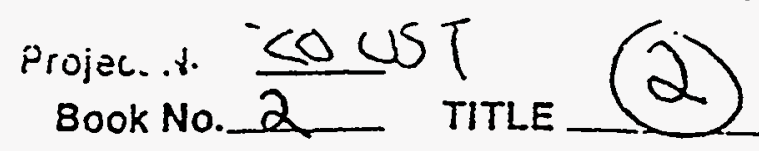

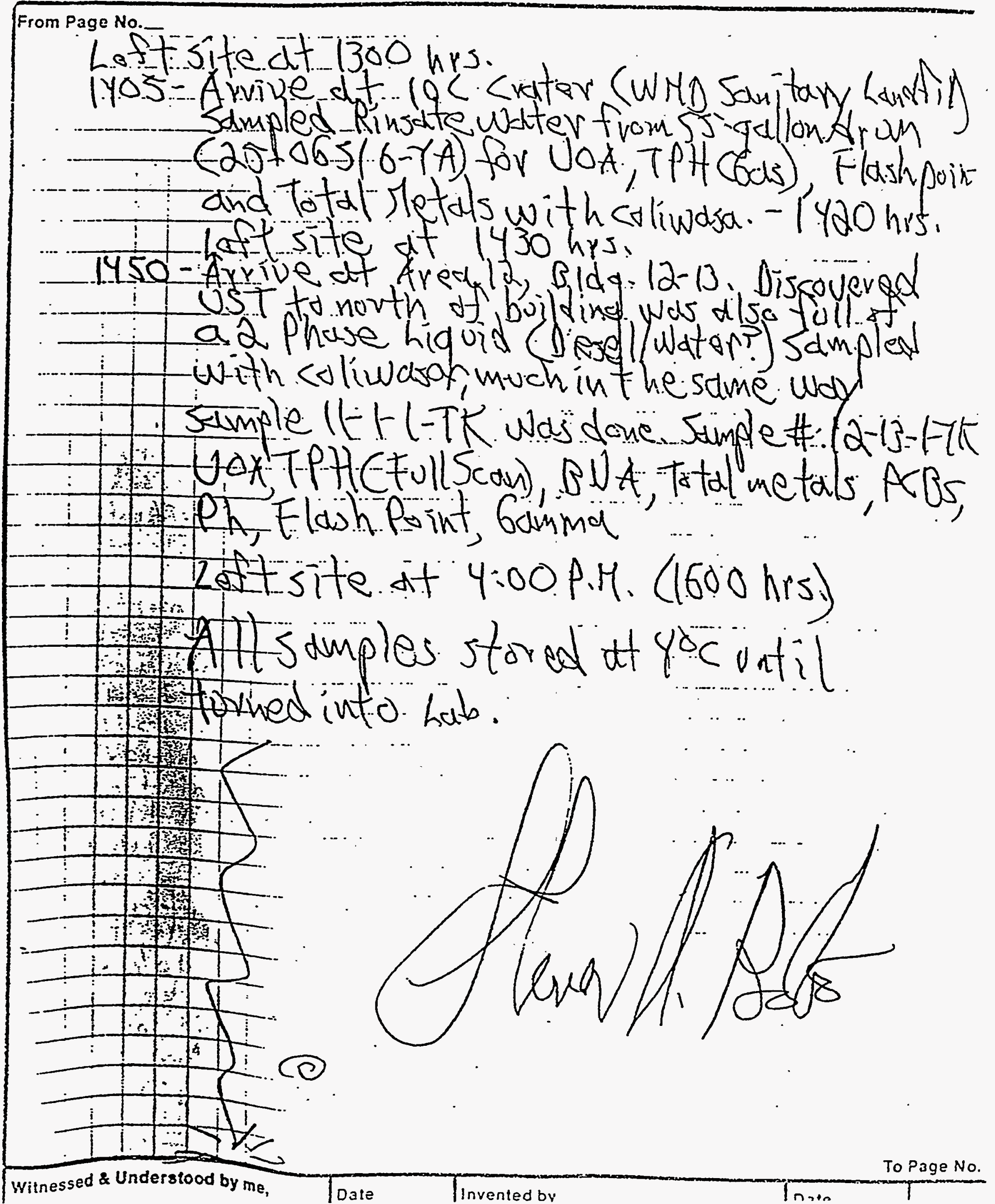


$9 / 21 / 92$

gery Dugar called D2M. Dare not- berlay, so el returimed his calle 5-7997. ch regends to the tank sempling acturity last Weds. for UST'S No. 11-2-2 ? 12-13-1 lt seens that ASD bie evough of the ail pleare tom MS/MSD, lut are laching enough nolue of water t Ren MS/MSD for BNA aralipis of the water ploses. I explanied to Gery hou the sumple crere taker fron the Two tanles ard how the scupler thaught there vora enaing

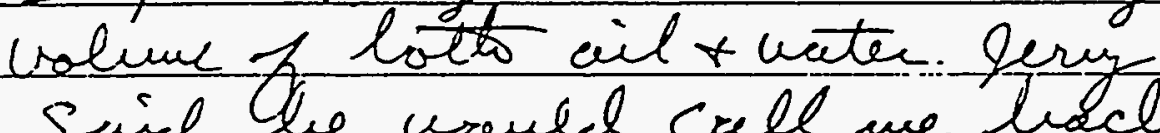
Said the vared call me hach. also, be sivid be lead a sapple from $12-13-1-$ TNK that lind 900 mils of ail +100 mlds of $\mathrm{H}_{2} \mathrm{O}$, and nue sypored t Le a vater sample. el Told hiv it unest he vismarled ot becanse witt that laye aront of ail, ard hav we sappled, it must be an ail sayple to he analyzed.

- Gave S.N Status of ASO insue as 
$9 / 21 / 92$

Derry Degas called Bach such teat they nay wot have enough iata t run VOA's ar PNA'S. OAtes lo vi at Battles for loot $11-2-1 / 8^{\prime} 12-13-1$, the hest cause for. 1 . bottles nos $30 \% \mathrm{H}_{2} \mathrm{O} / 70 \%$ ail mixture. 1 by have enough ail to rival analysis plus

MS/MSD is for ait. Since they dent bran about $\mathrm{H}_{2} \mathrm{O}$ they will analyse as munch as they cos, an will call we of they have a problem no not ensoul dater. The need. at lest 4 liter of $H_{2} O$ for each 
9/221/93 hat w/ Steve Kelleman e offin. Hut ip nith Bofe Bull + tave Gagne (pollis diven) c. P8:20.

$$
\begin{aligned}
& \text { Tahker } 1 \text { W } 9 T<M 3 N 9<1021193 . \\
& \text { Dof E- 0822/Test Duft IV } 1092 \\
& \text { Co.Plut \#10.G6856 }
\end{aligned}
$$

-Itef for a/ra e ? 11:30! Cenviel. $a / 2+-12: 30.18$ moluct frem 12-13-1. Sluch w. a dipetich, the teich contur lex then 3. fiet of rodupt. 1he dwin uscel a $/ 1 / 2$ striger ticacen all tes ung to foetap. Brciuer estriates $\sim$ loor gals of mofuct. Ituch tenh agen. no spalucti clescinered. 


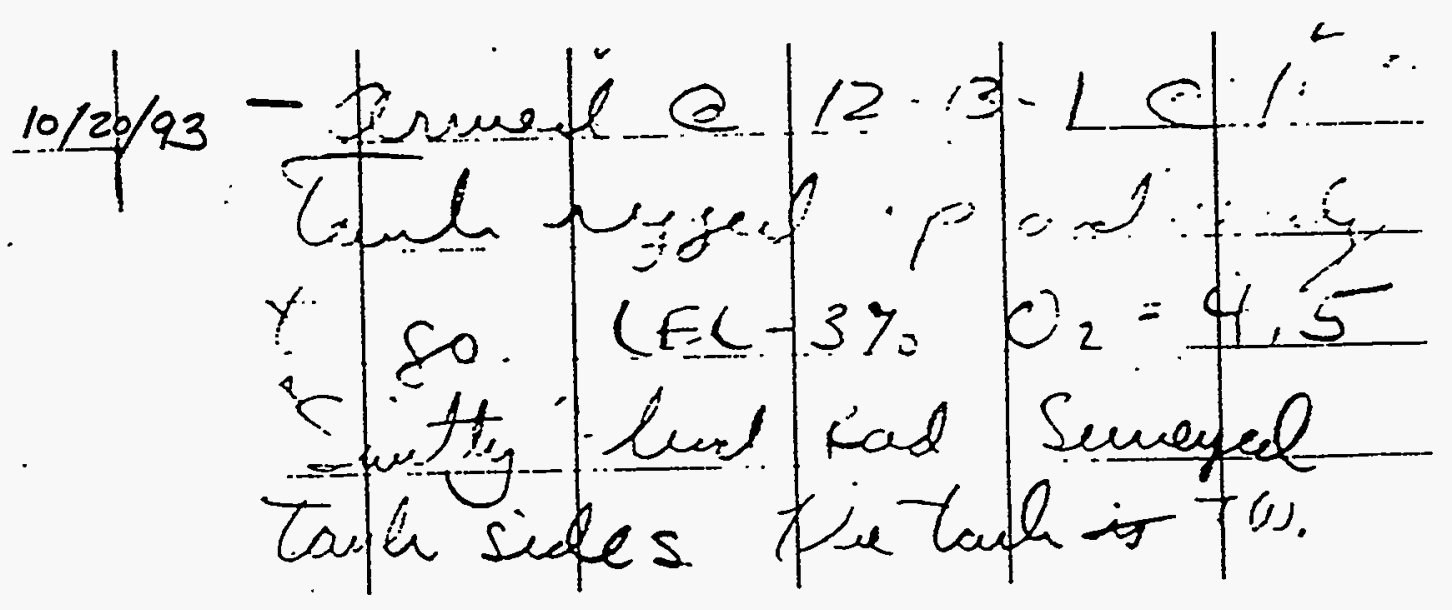




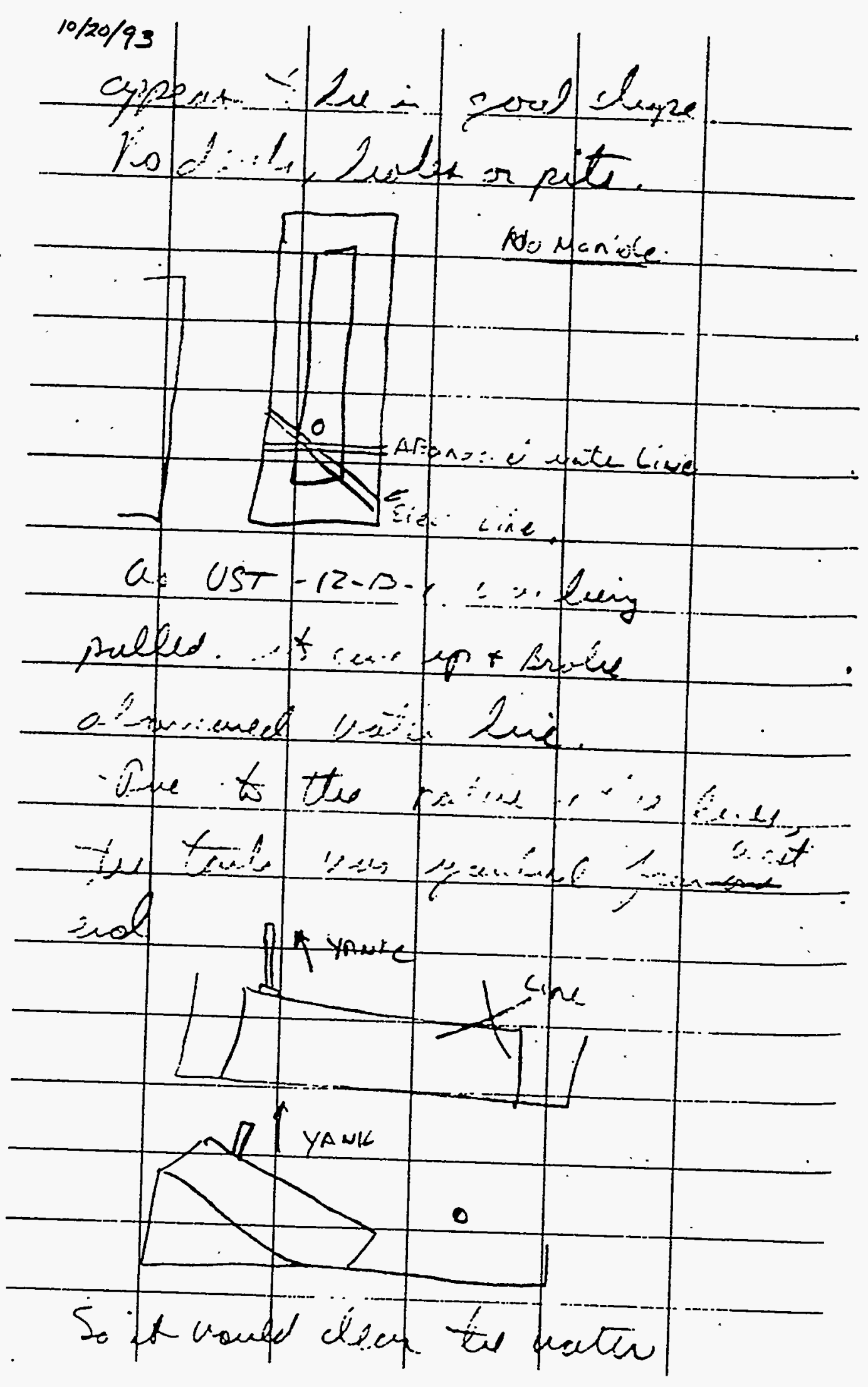




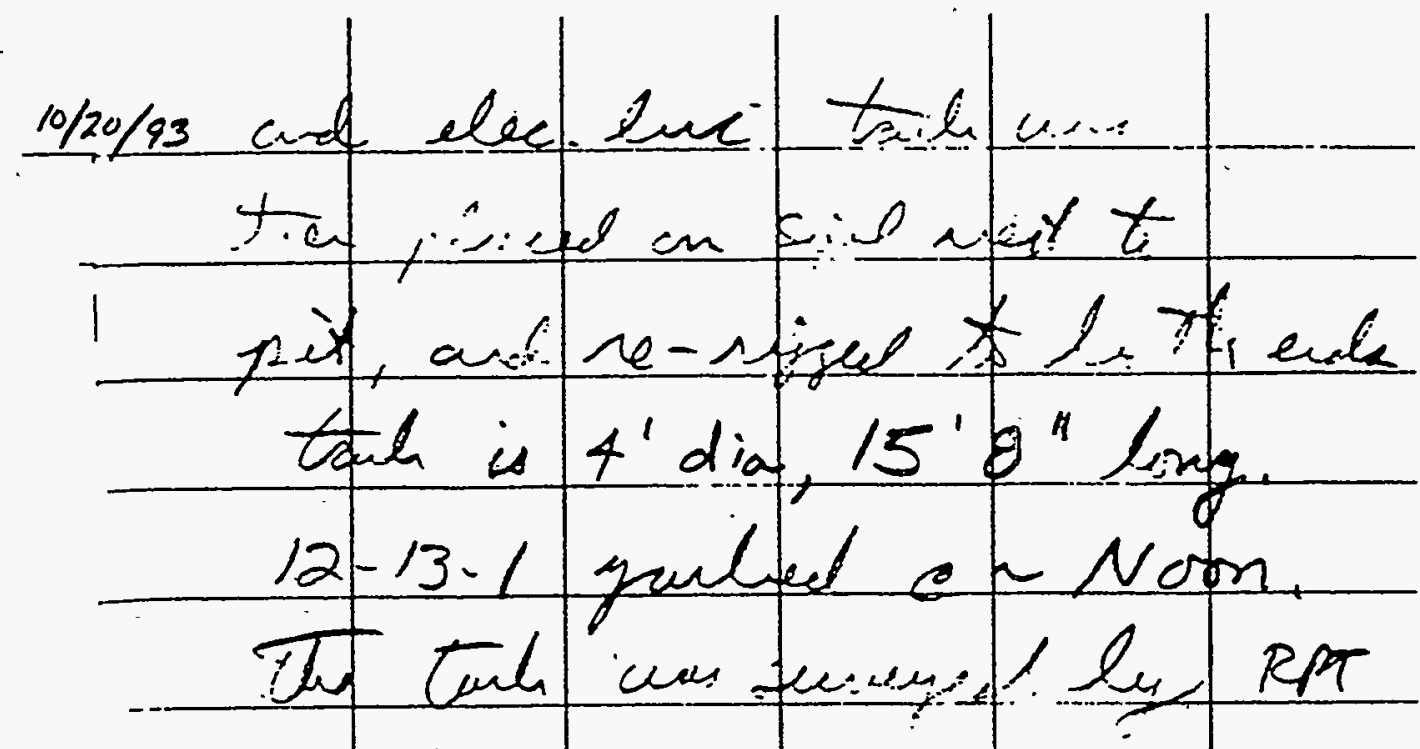

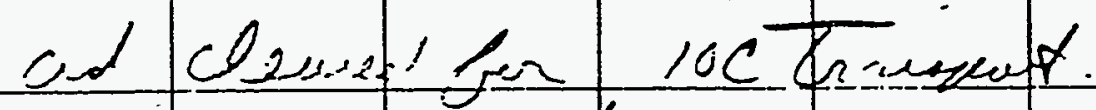
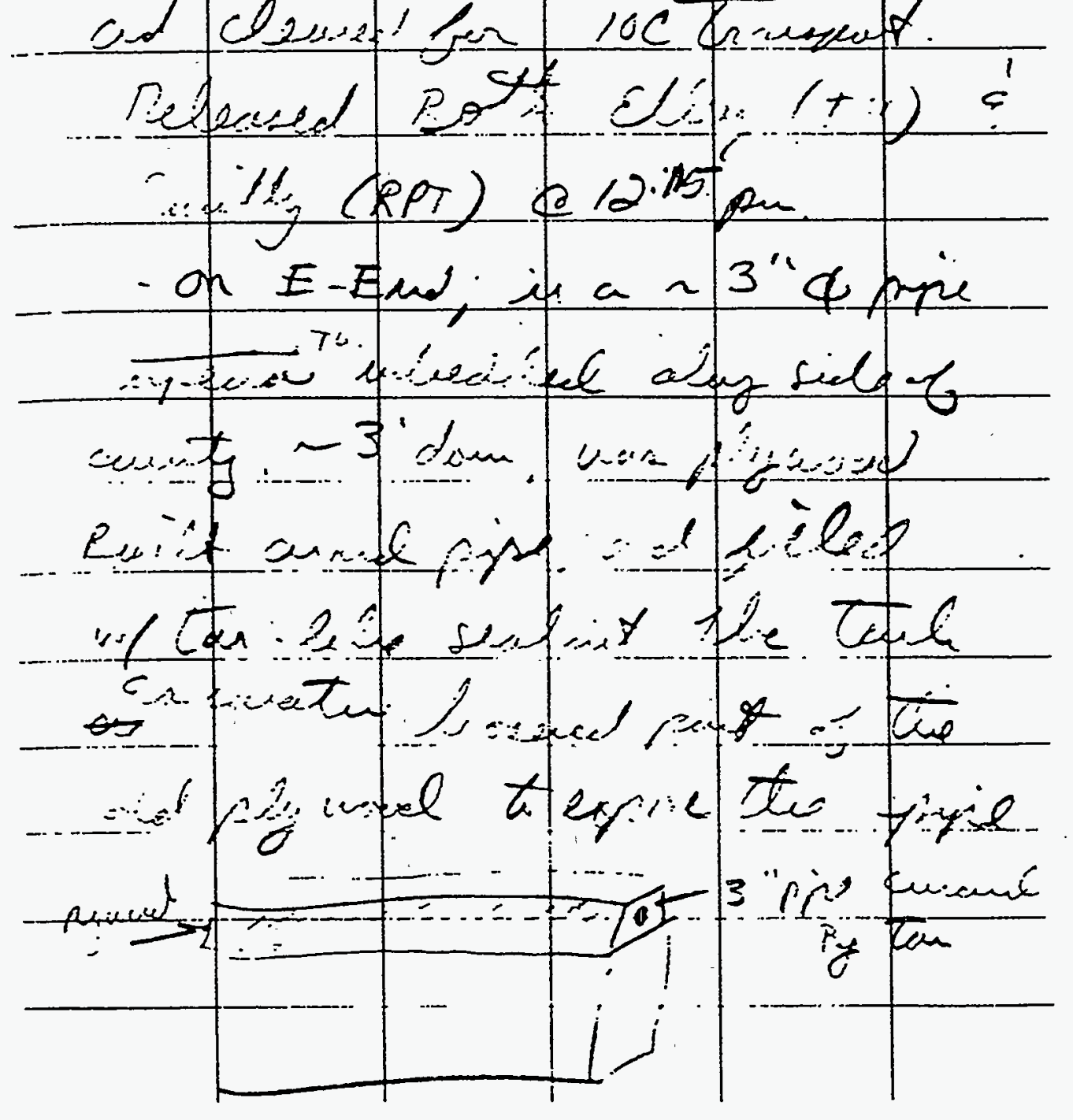


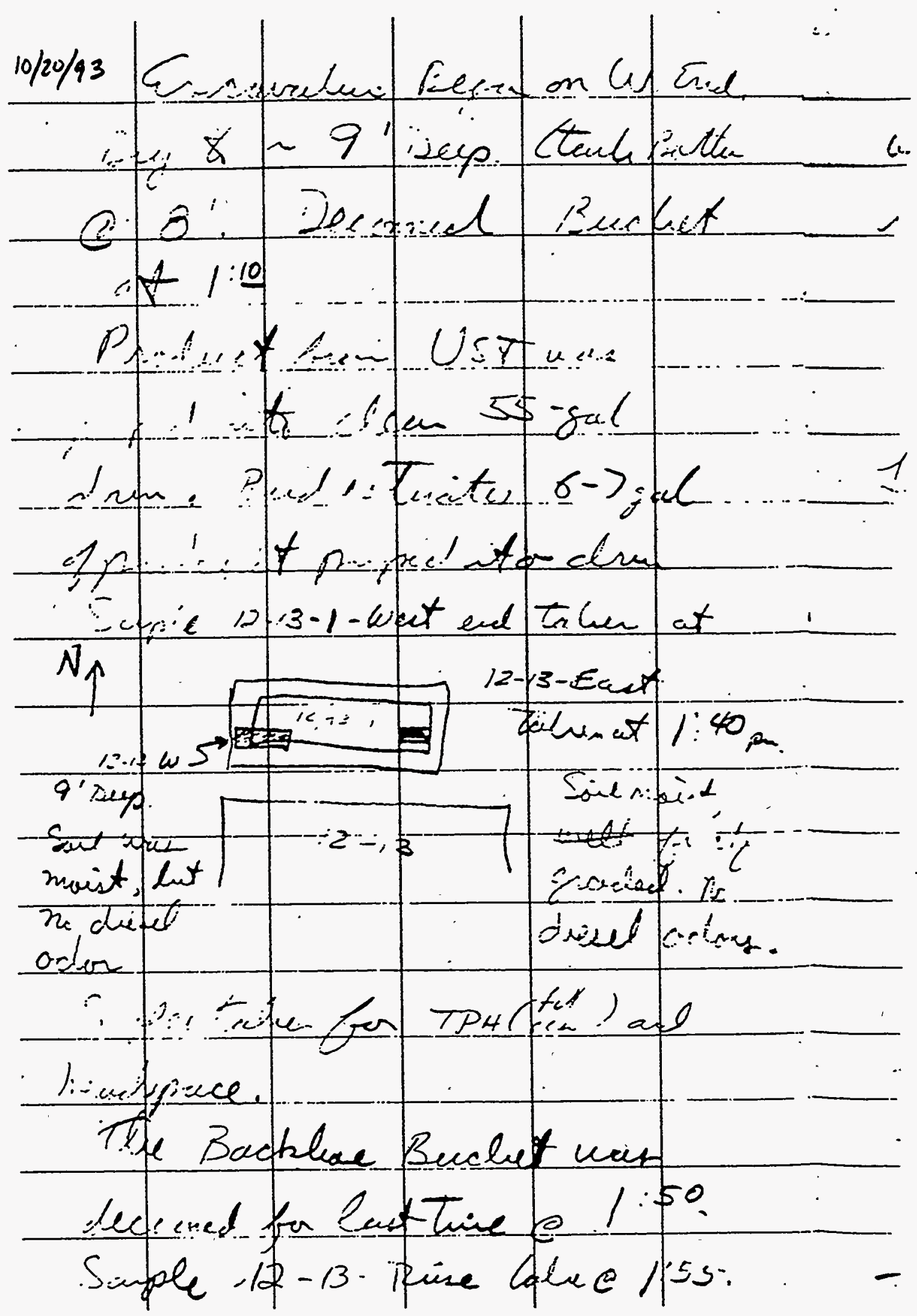




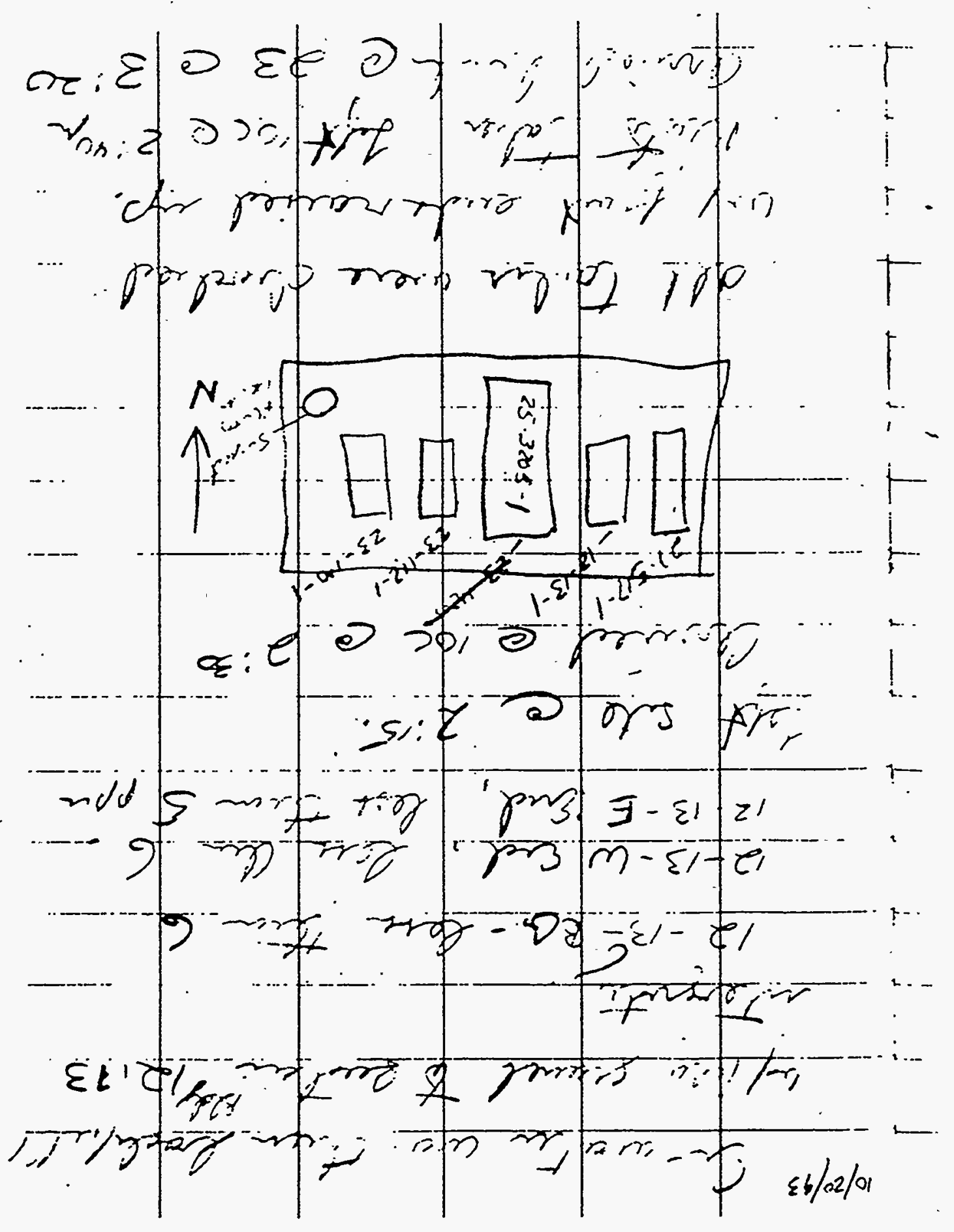




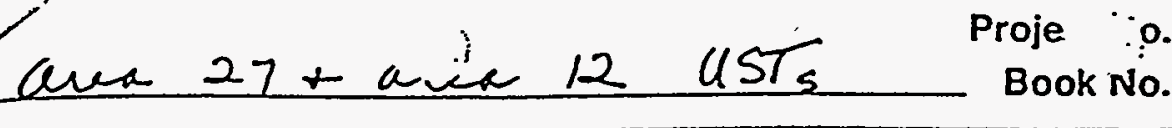

19

From Page Nolgl Wel reshry, Octorher 20.1993

at the request of Tom wilezat, ERS, futrueiy met hem of Bedg.

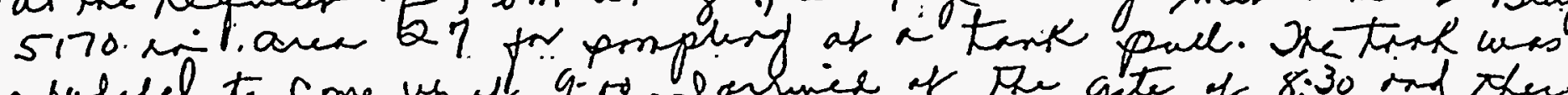

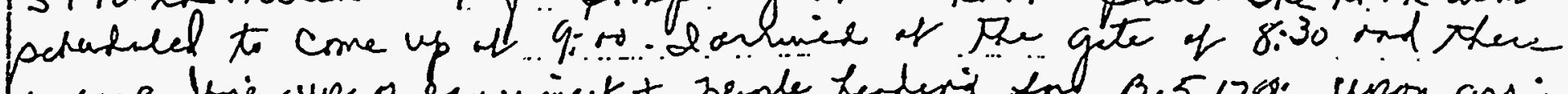

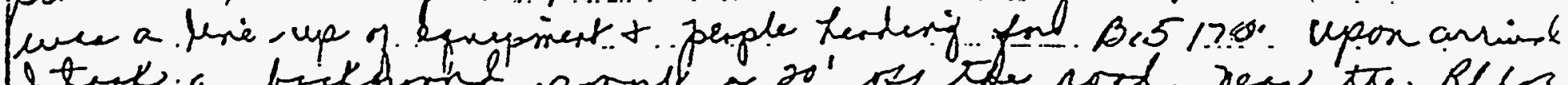

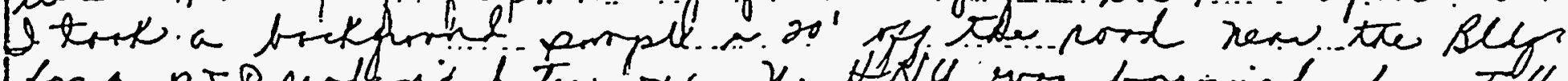

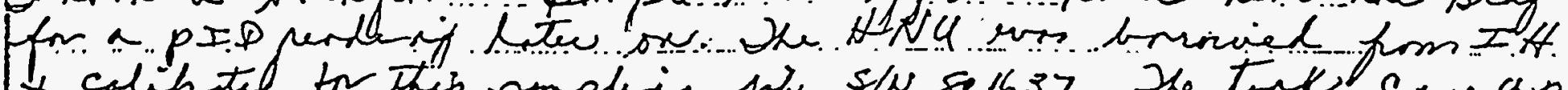

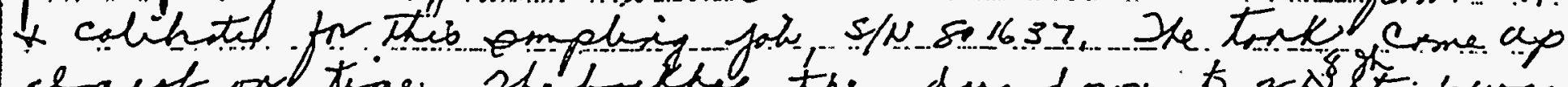

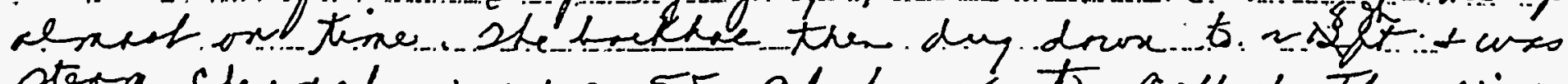

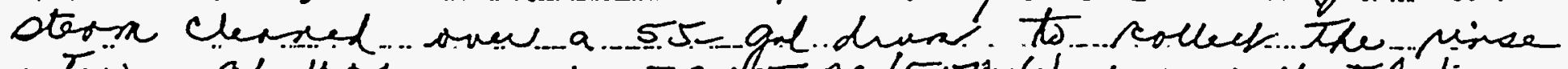

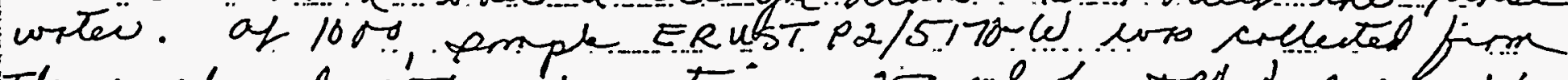

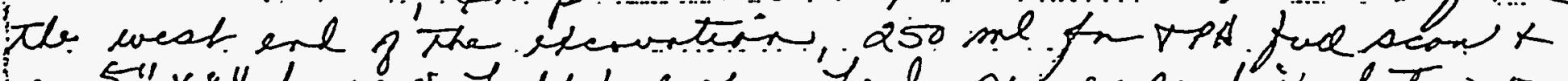

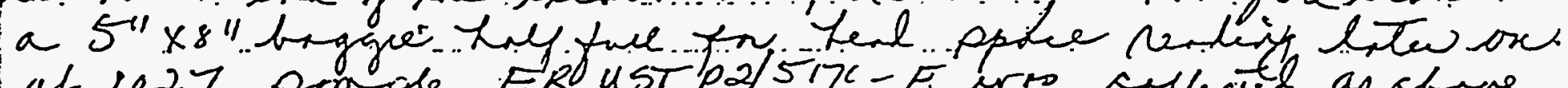

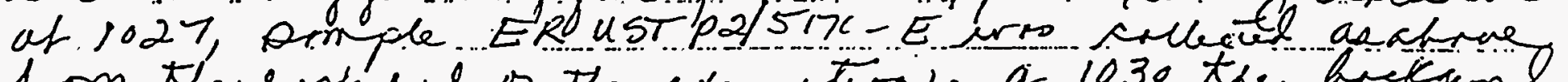

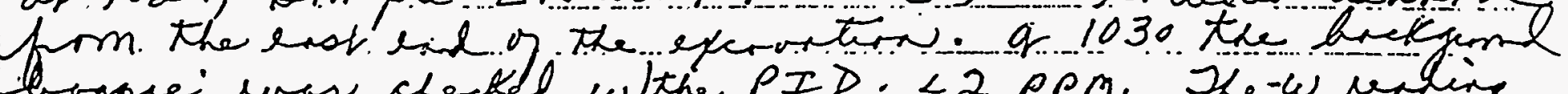

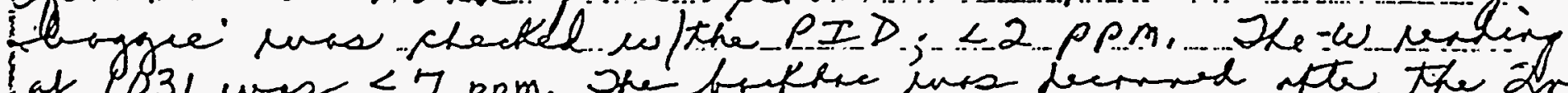

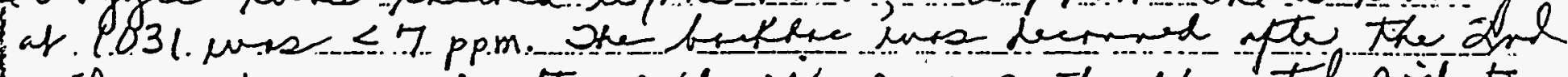

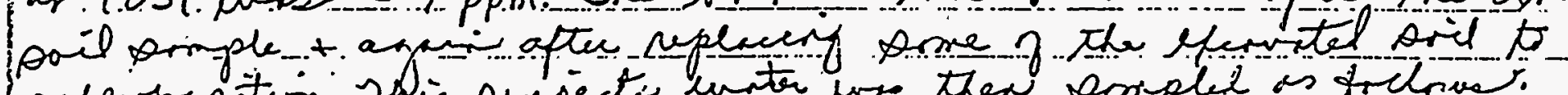

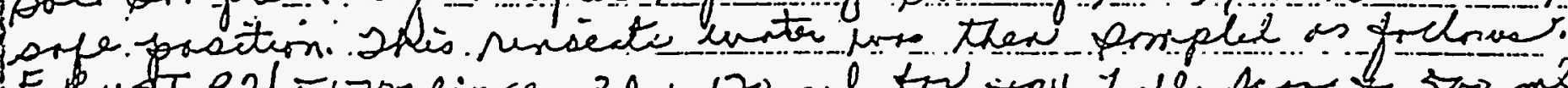

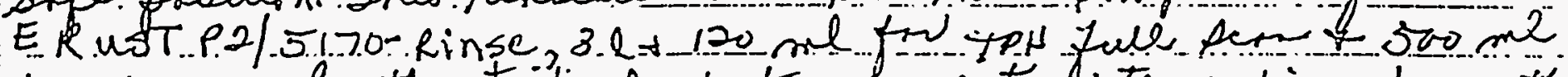

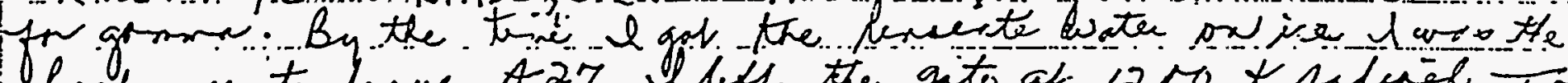

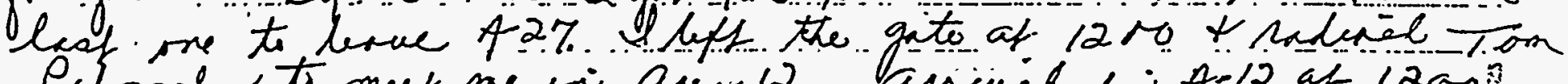

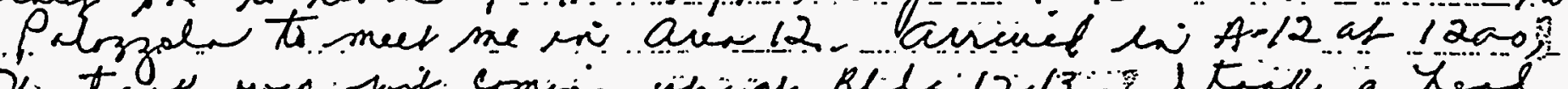

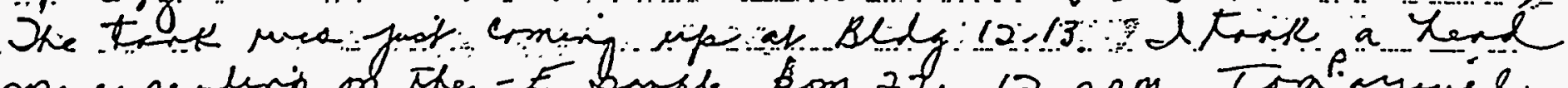

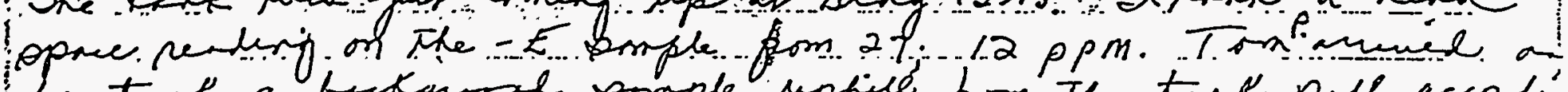
the tark a bukgral omple uphile fum. The tork pull acendir

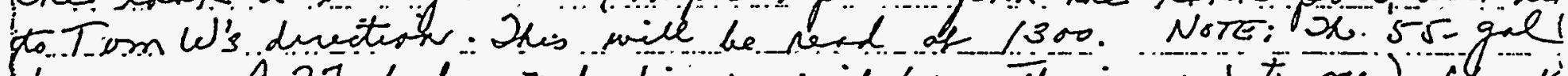

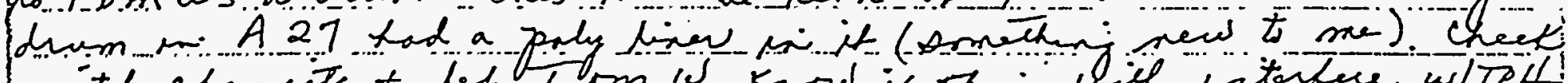

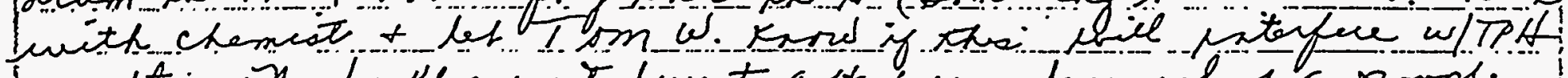

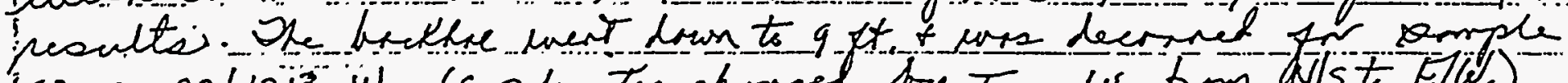

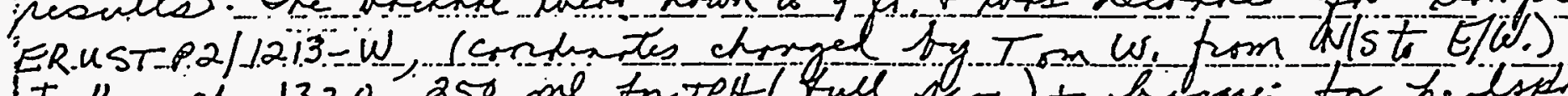

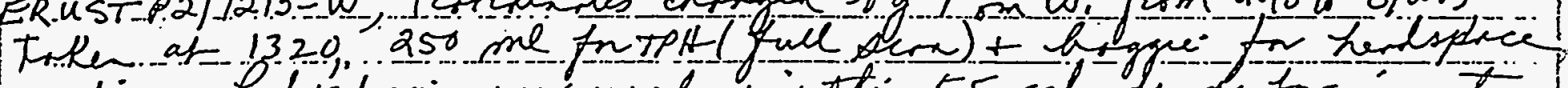

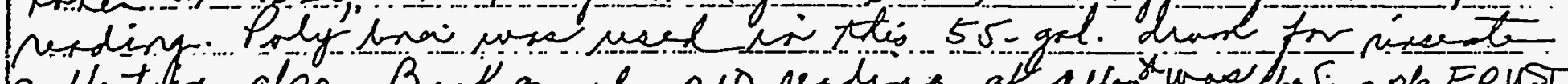

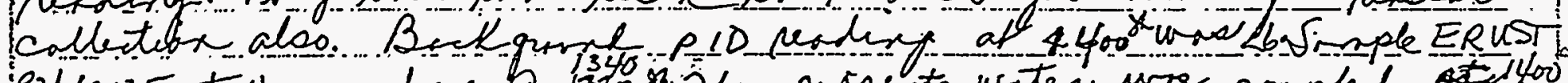

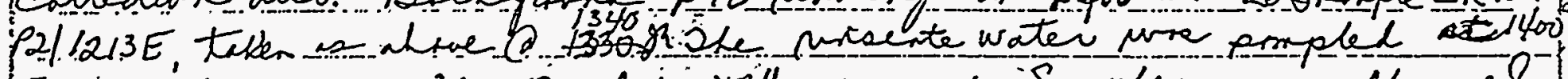

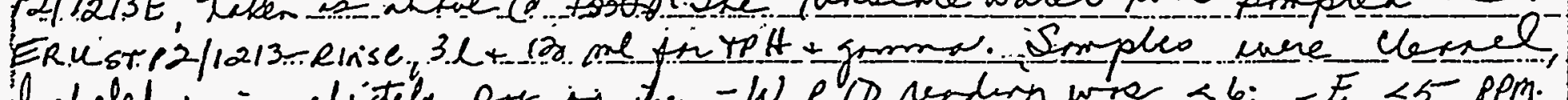

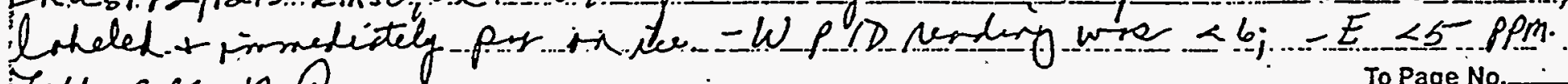
Keft area 120

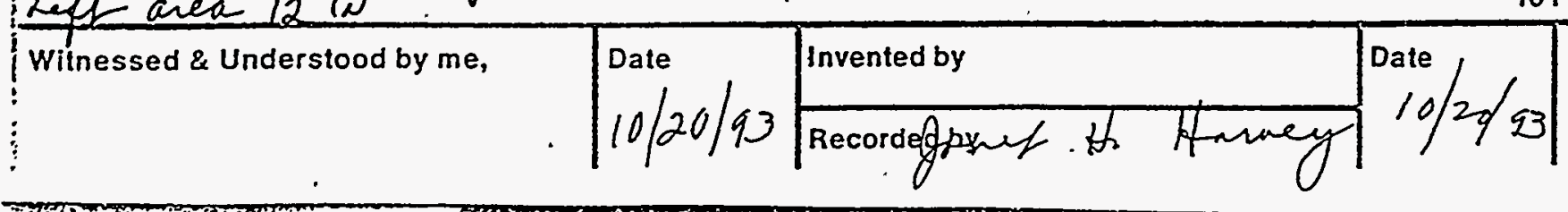


Project .10.

TITLEA23,A27,A25, A12 Piles

Book No.

6:

From Page No. $11 / 09 / 93-0800=1400 \mathrm{cos}$ calm

Keguestor . Jom Wilesek

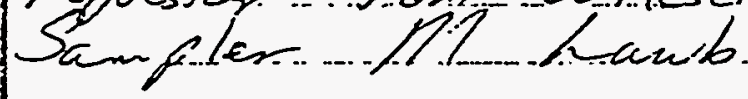

All samples composiferi from points indícatu by " $x$ " marte on piles.

- Various soil samples of dict piles axound site pleperesuadih added A... All on ice isinedrately. ve odd smells ox discoloxation on - any sauples Typical dist. appearance. All sitis checked revioush

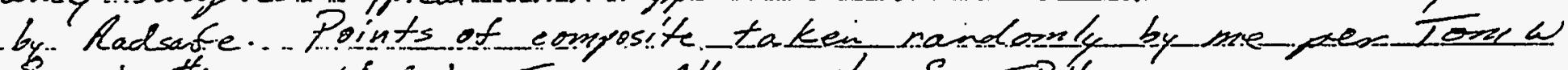
Sauple \#s providex by Tom. All samples for TPls

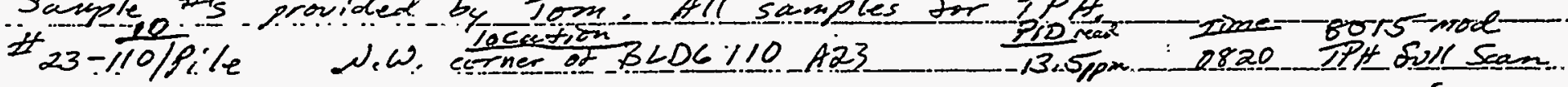

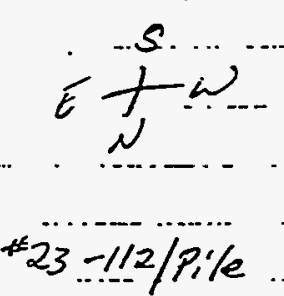
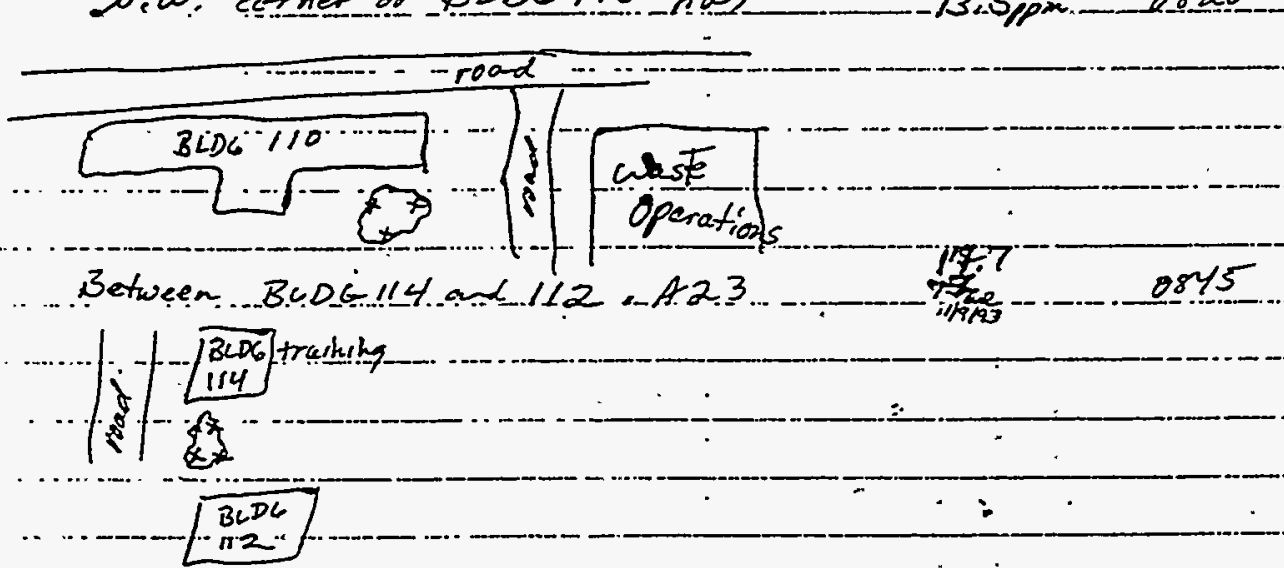

\#

$$
E=\frac{5}{5} \omega
$$

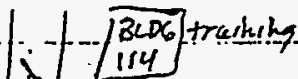

$$
3 \times 26
$$

$-12 \div 8 / P ; l_{0}$

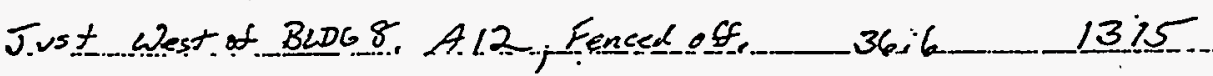

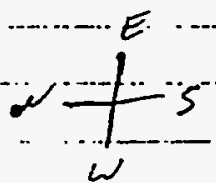

$+12-9 / P i l e$ behind. West of medicul 3CD6.? A12 $29.0 \quad 1250$
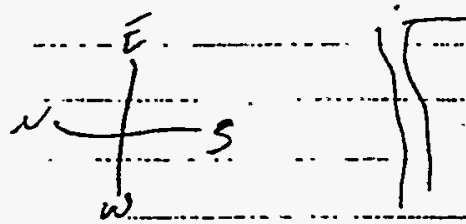
3006

$\# / 2=13 / 0$ ile

behisda west of BuDC iz-43

14.1230

TPA-8015mod diesel q...qas...

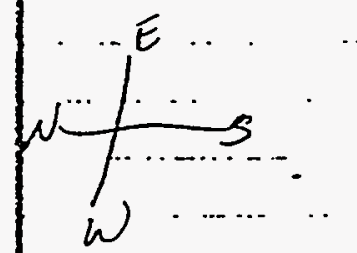

T2DC

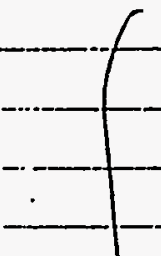

(2... 
$11 / 9 / 93$

- adrinede 12-13-1 at $\simeq 12$ :30
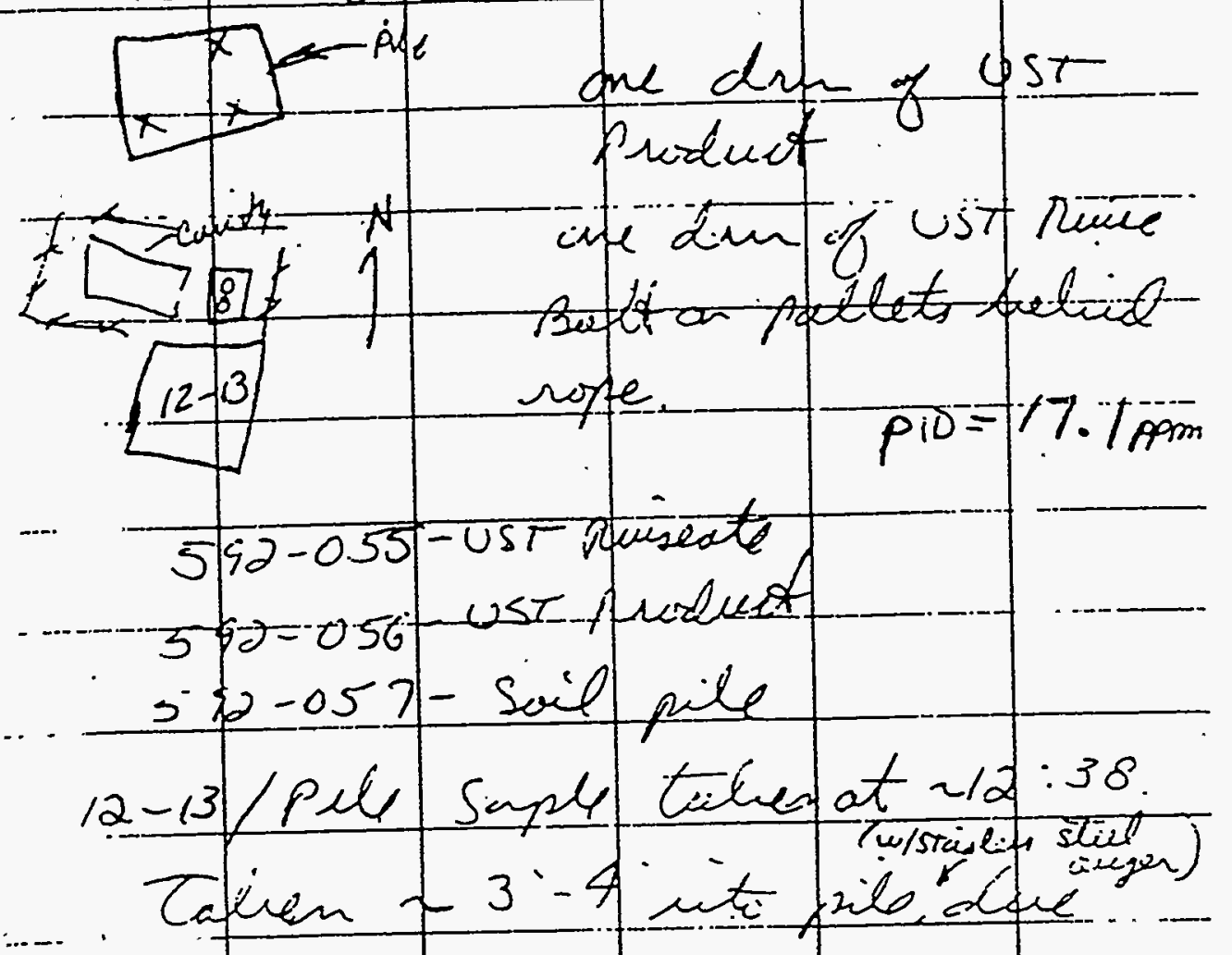

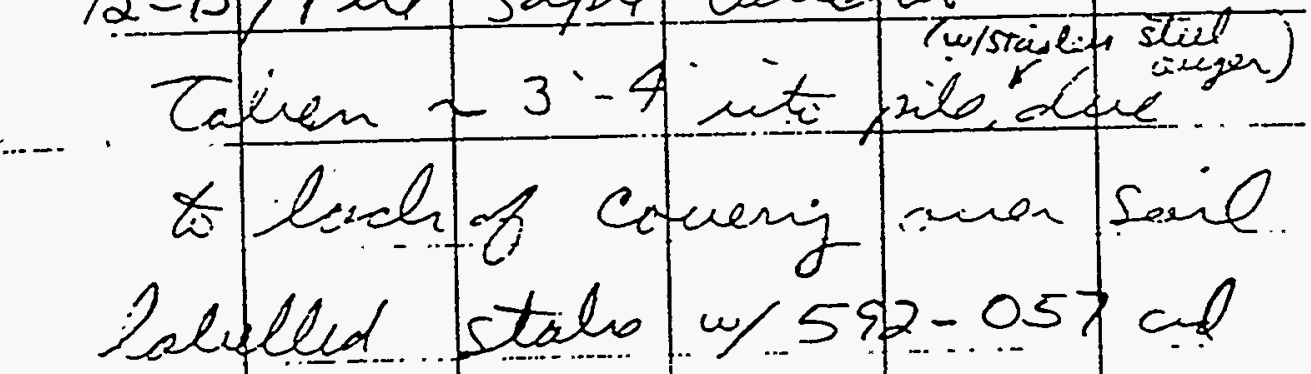

.. stukh i frond r fint t. nelo

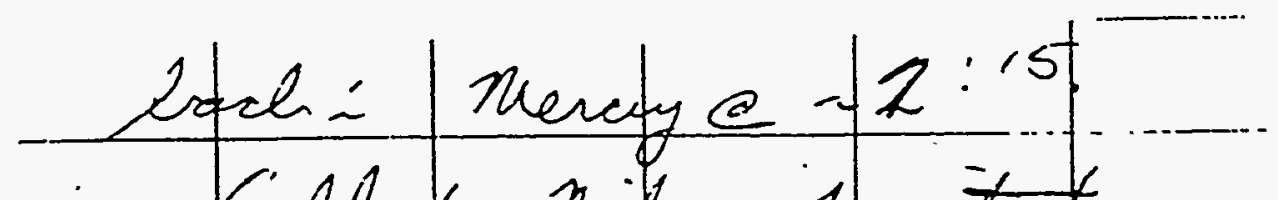

- Callek Nitu Ipstat, cofel hi hewatd tru veets $\&$ live Soid pils reconeref 0 (2-13. 16 safiel coutet le chen ty enel of cuelek. 


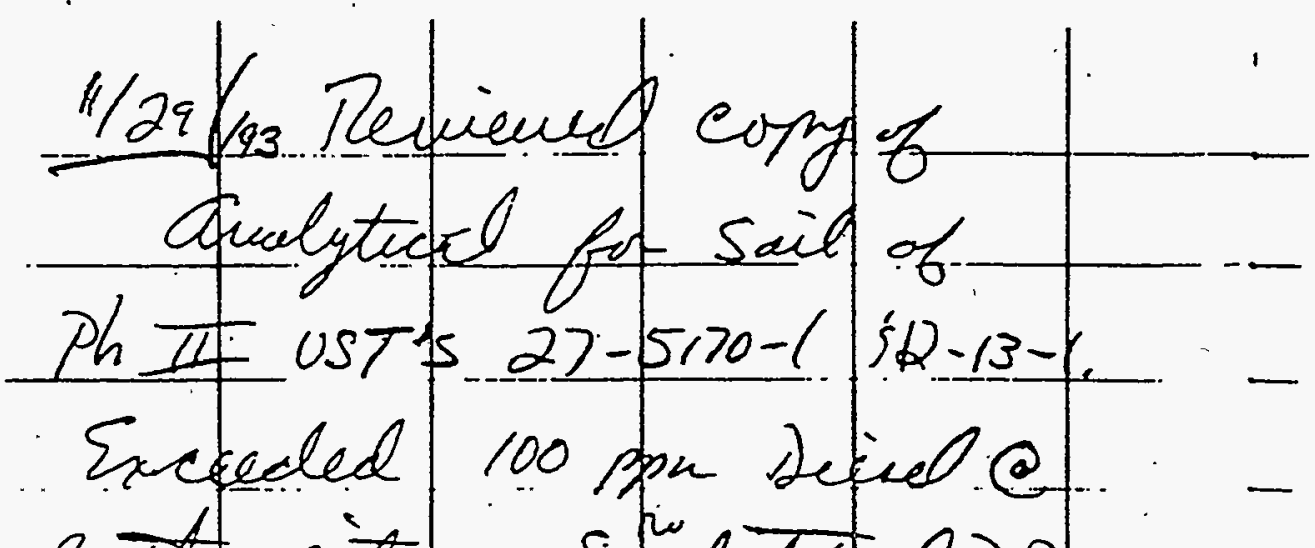
Bota. sitex. si rotpred oAn -

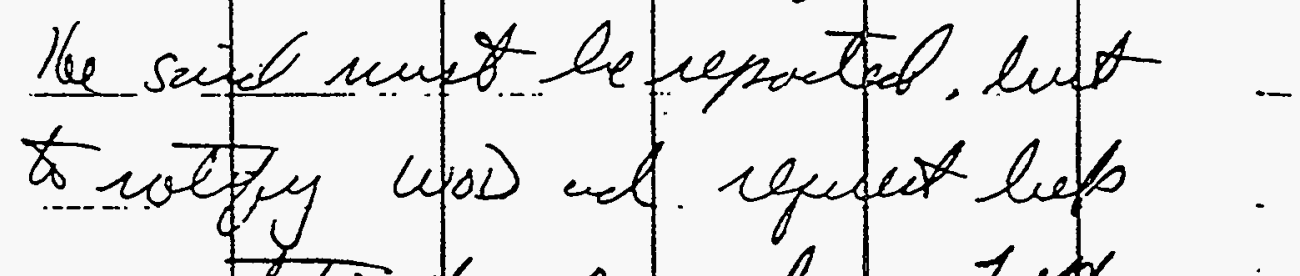

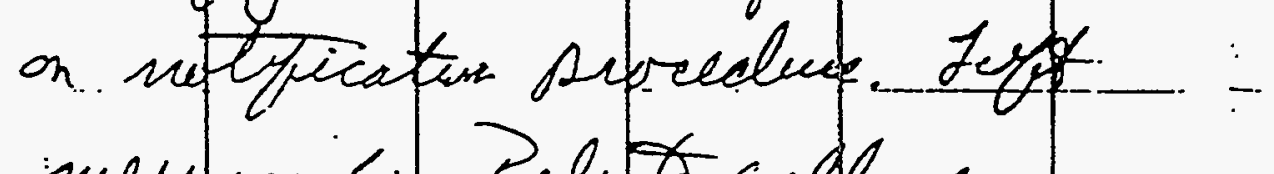
mentaye for Rolito call me

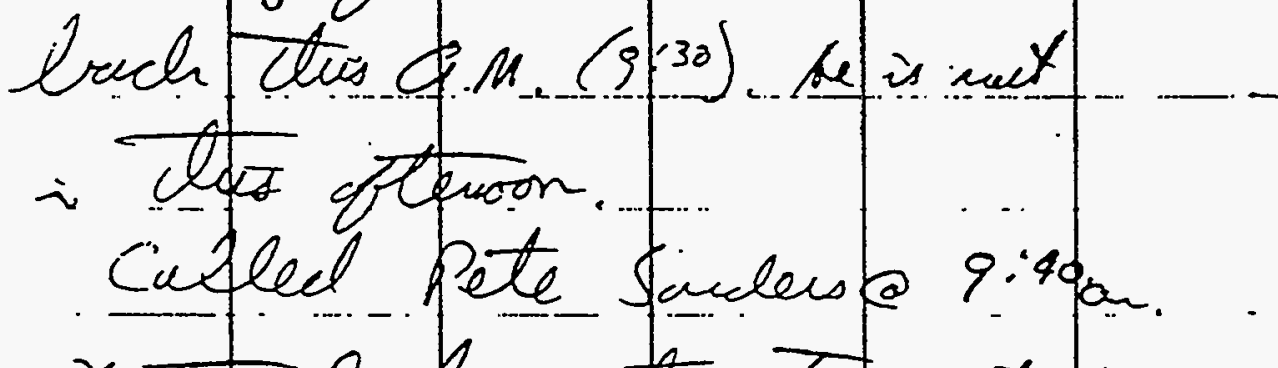

notped firyte tuo ste saief for if foretering.

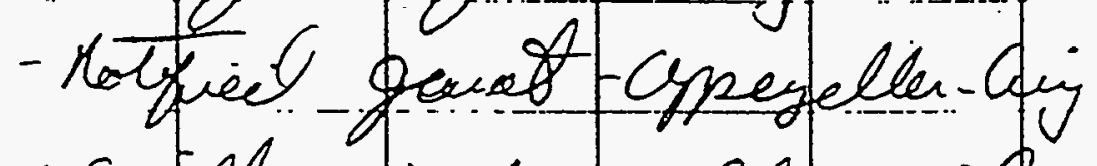
of spill in A. st se said she would lile the ifouter... to sfow ry - the neebly ace. send ther. 
$11 / 29 / 93$

- Dom toto ho teal Claytar Barrour of Dof 1 NO

Live nefro tootfy her

Clled Claytar Barsuce 140 to notity hinf ust spele.

cav dine mys: .

Fured copies of the zepiortr.

t: Pete Suller e 50838

Danct wring C 51113

claytem Banawe 56242

folm Doduertan e 56392

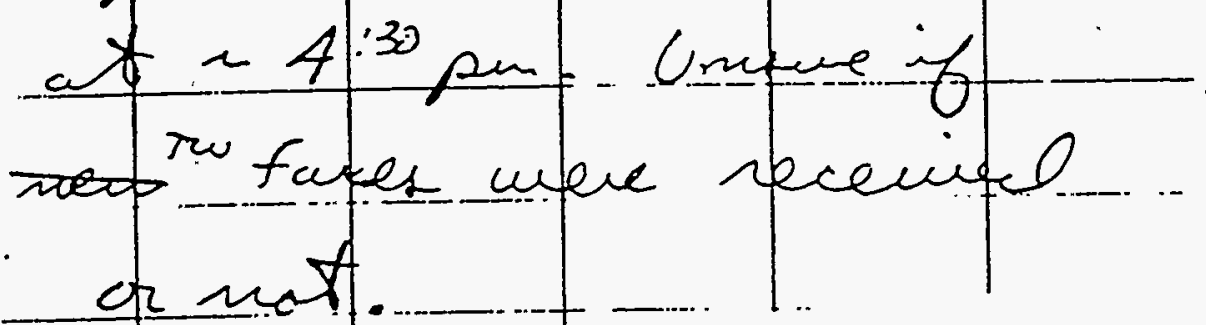

- ormot.

- Updated Bol Dooffe on to

tho distamenel sple 0 US

$2>-5770-1: 12-13-1$ 


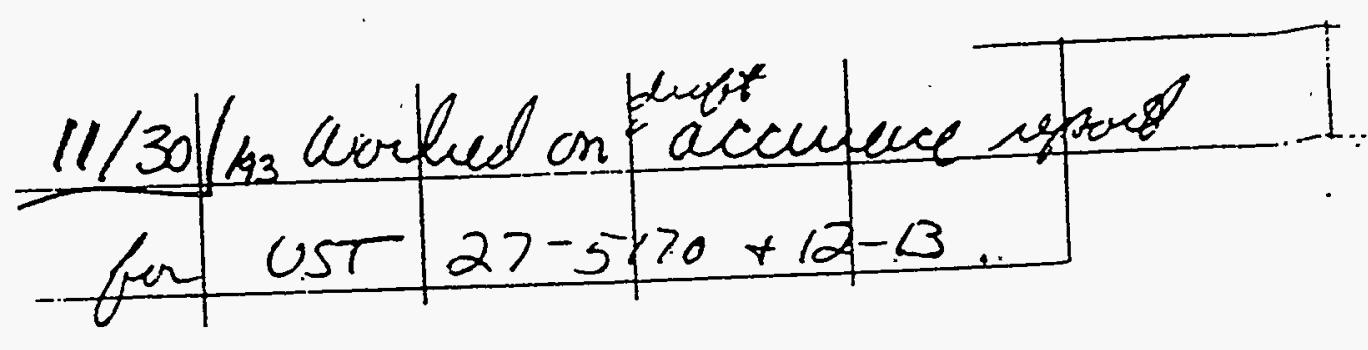

Reseacted Facielty nyt's

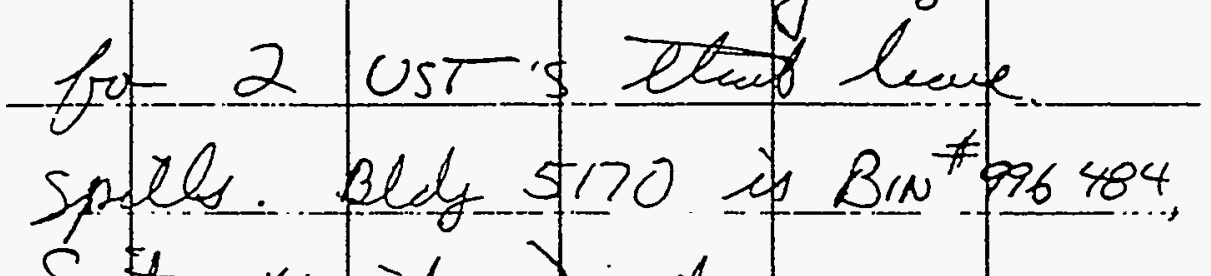

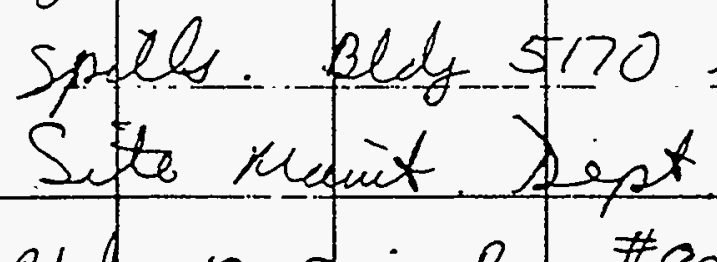

Blogy $12-13$ is Bre \#998690, Galesin. Custertiat Food Serince Rept Rerequelued Bored on taculity

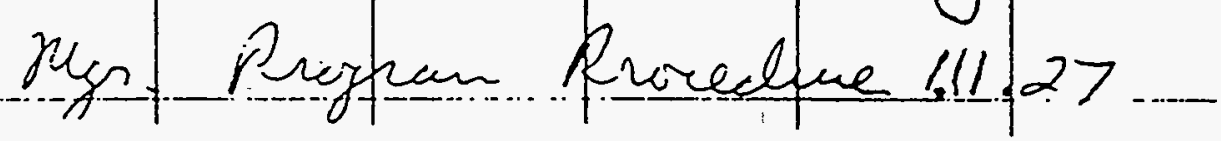


$1 / 18 / 94$
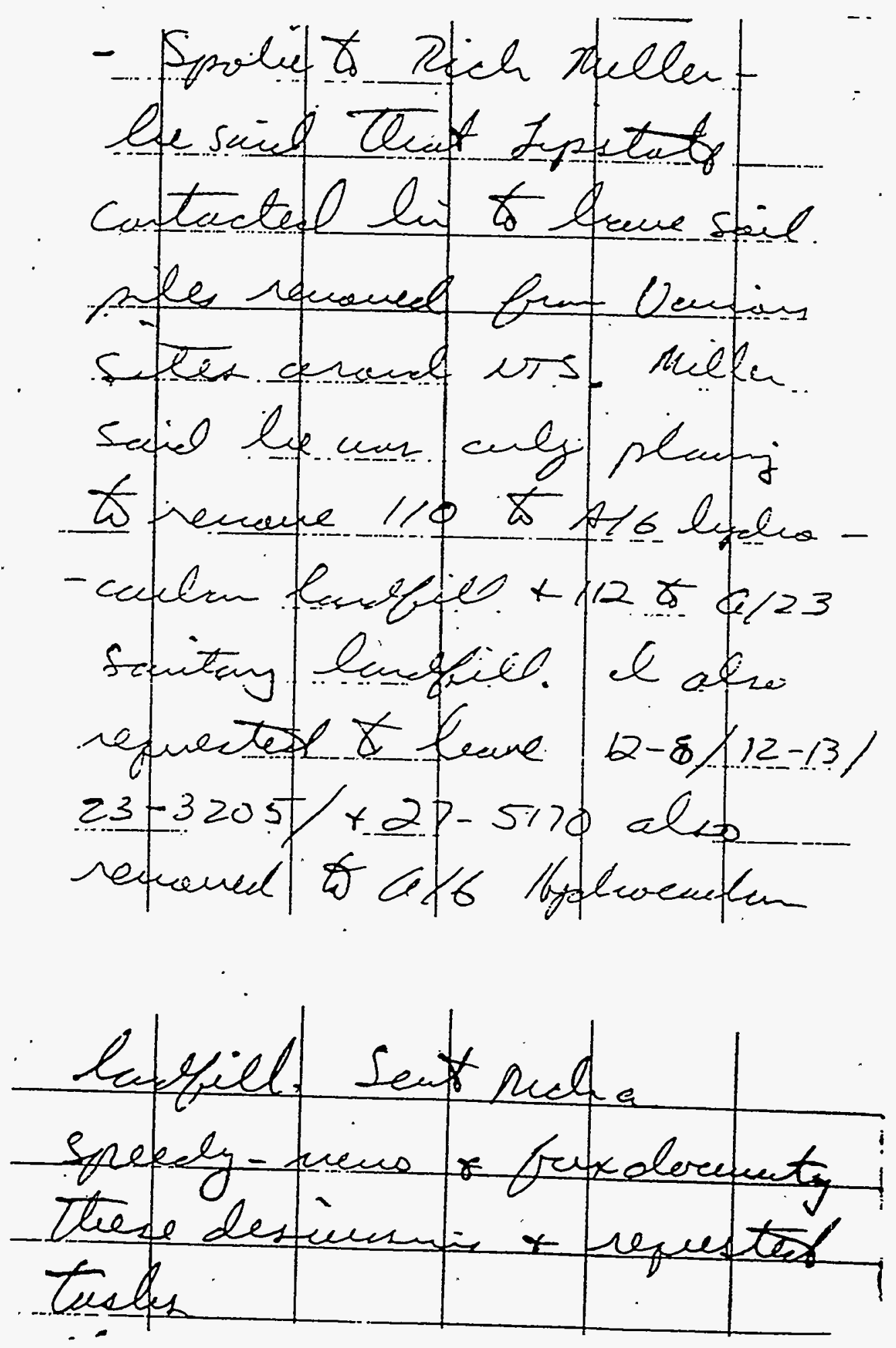


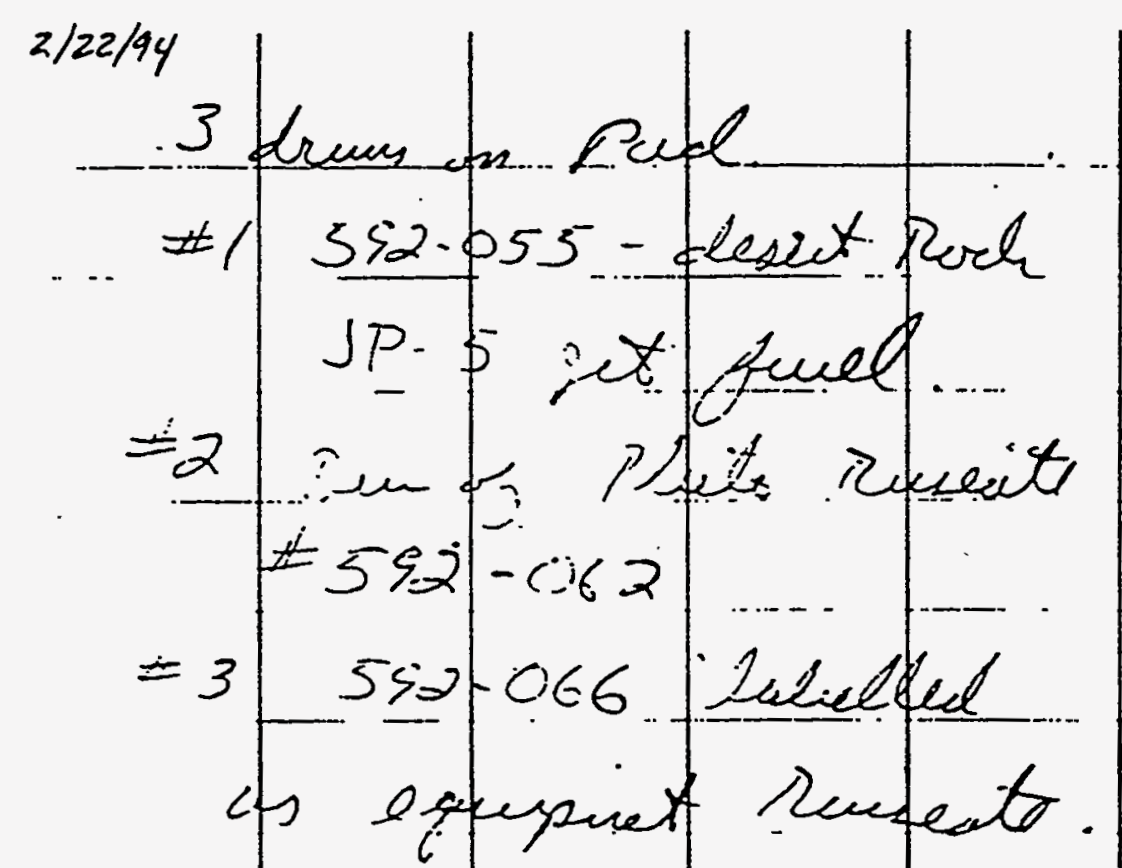

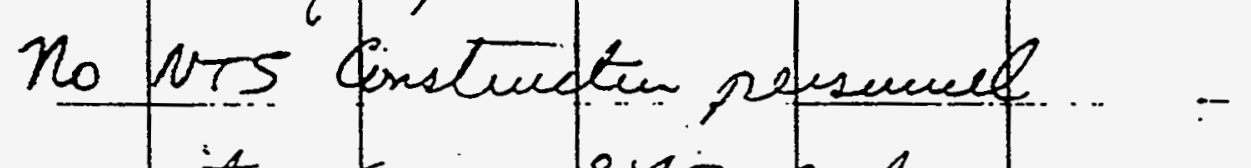
$\mathrm{cm}$-pite des if 9:45. quey Stew soltor nfuef. it. - 8:46. Eliery tonissa CIL Shaved ip Confat Lewe

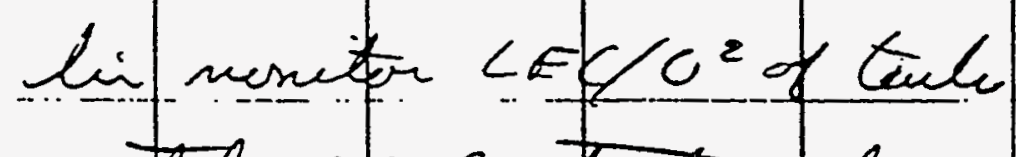
cutel its custucete shous -p usit the th trugh. dupictech tuils - all aue granted all are topict with to

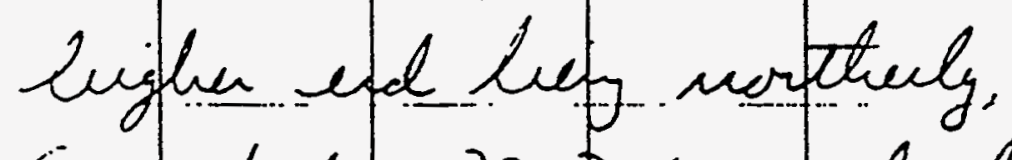
cracest for 22-Dra-3. kiluct 


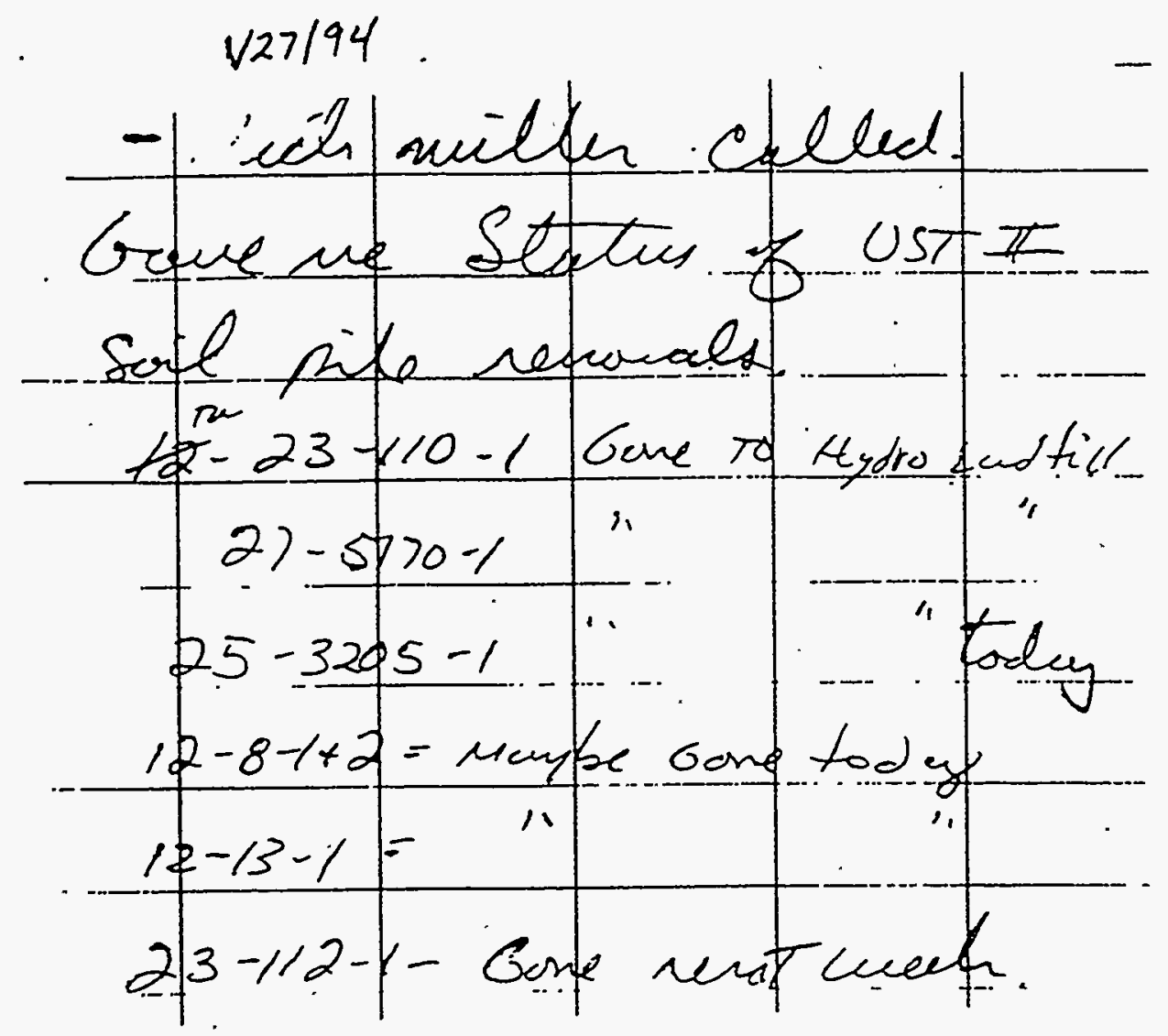

$2 / 2 / 94$

- Rich pillef called Quea of fust weel pile $\neq 23-12-1$ lev sidhed yp toduy 


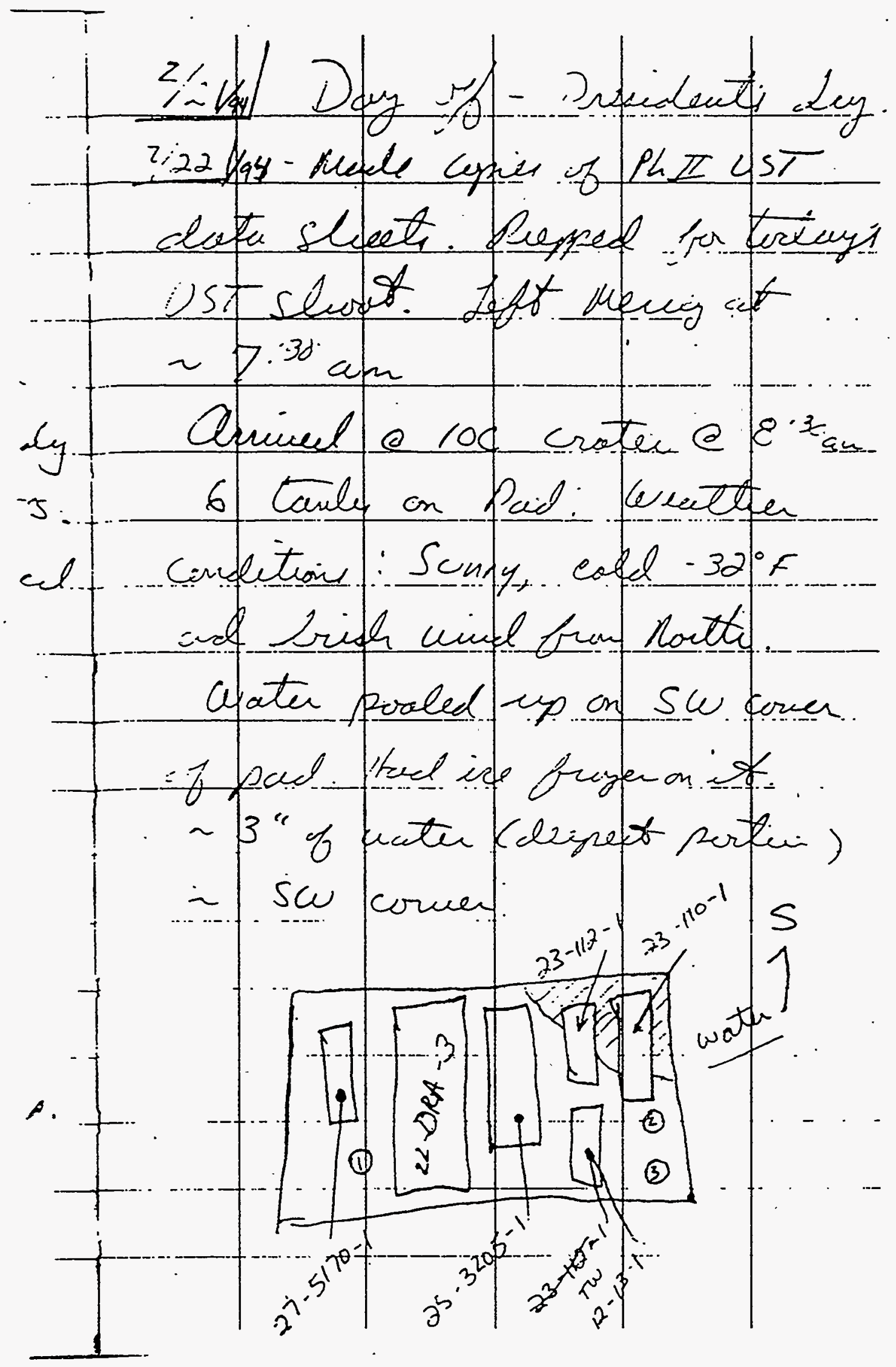




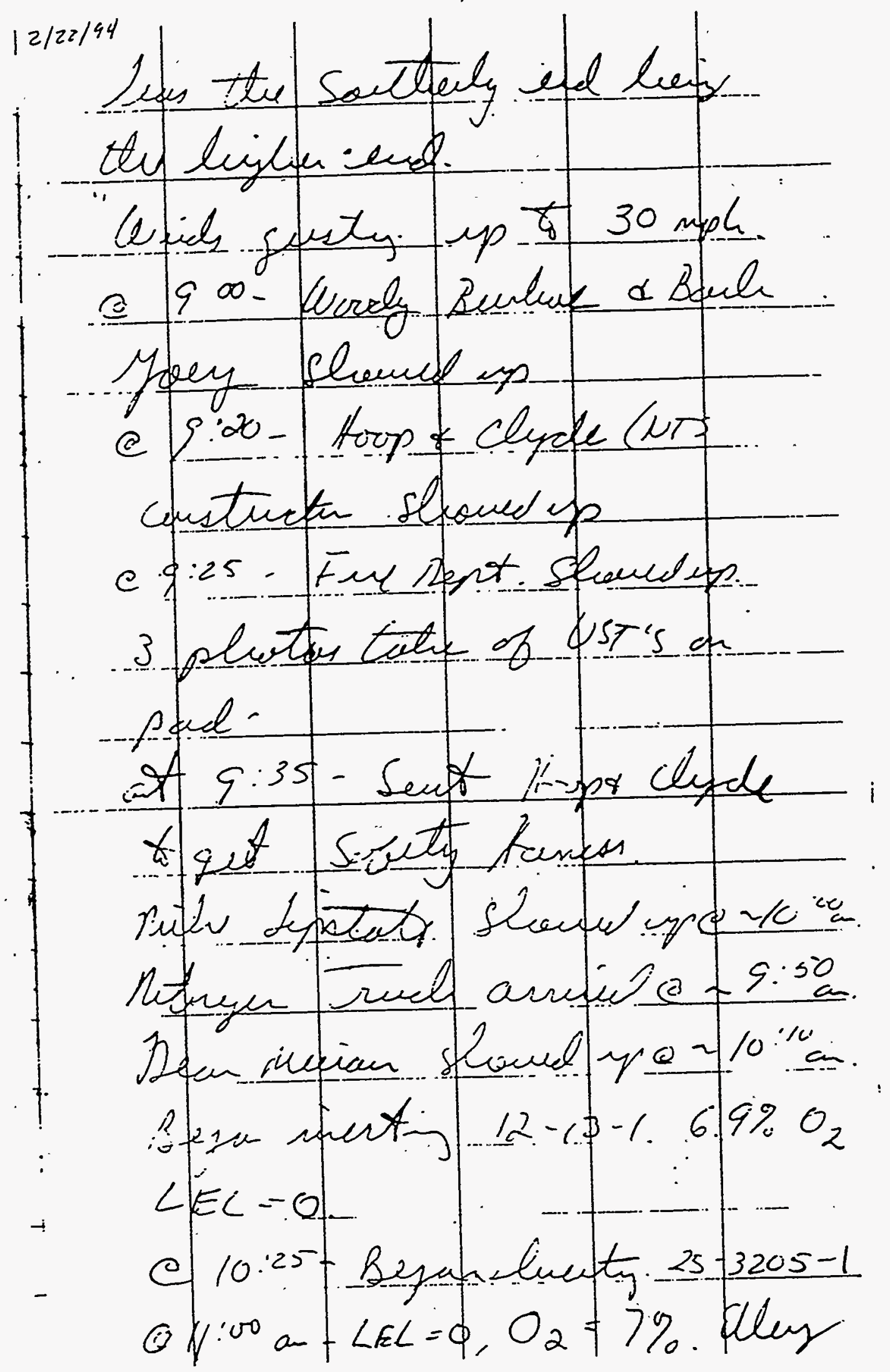


$2 / 22 / 94$

concurned it use 0.14 .

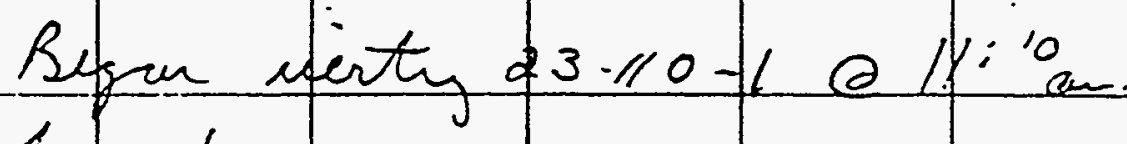

7 sished 23-10-1. $\angle E L-0$,

$0_{2}=6.6 \%$.

$N_{2} t=$ all tumbir seaked mott

tope a sluged A penint

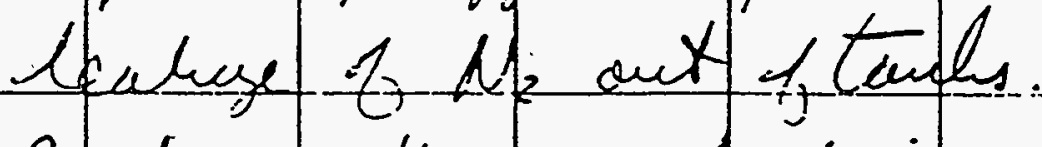

Bubrua foen asted if

ue comed stios dy tomanas

* Lorl ane cong of dnept nspectiv repot sle will

pepare. Tolf her $l$ wid

suel stew B. A torah t

it.

$23-112-1$ frumber : 11:20

$L E L=0, \quad O_{2}=6.8 \%$.

1hayon + Dare sherpe shounel

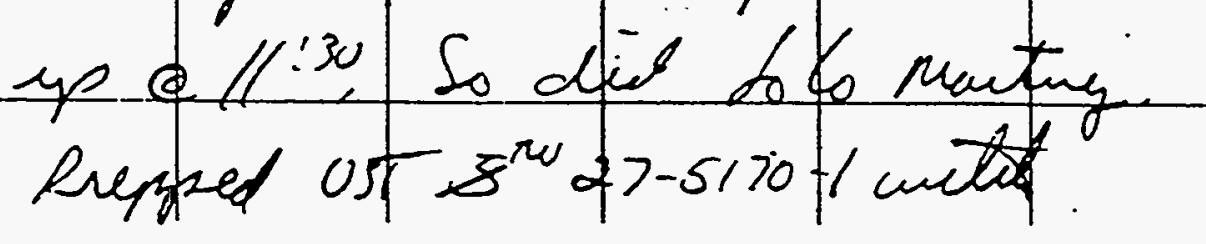




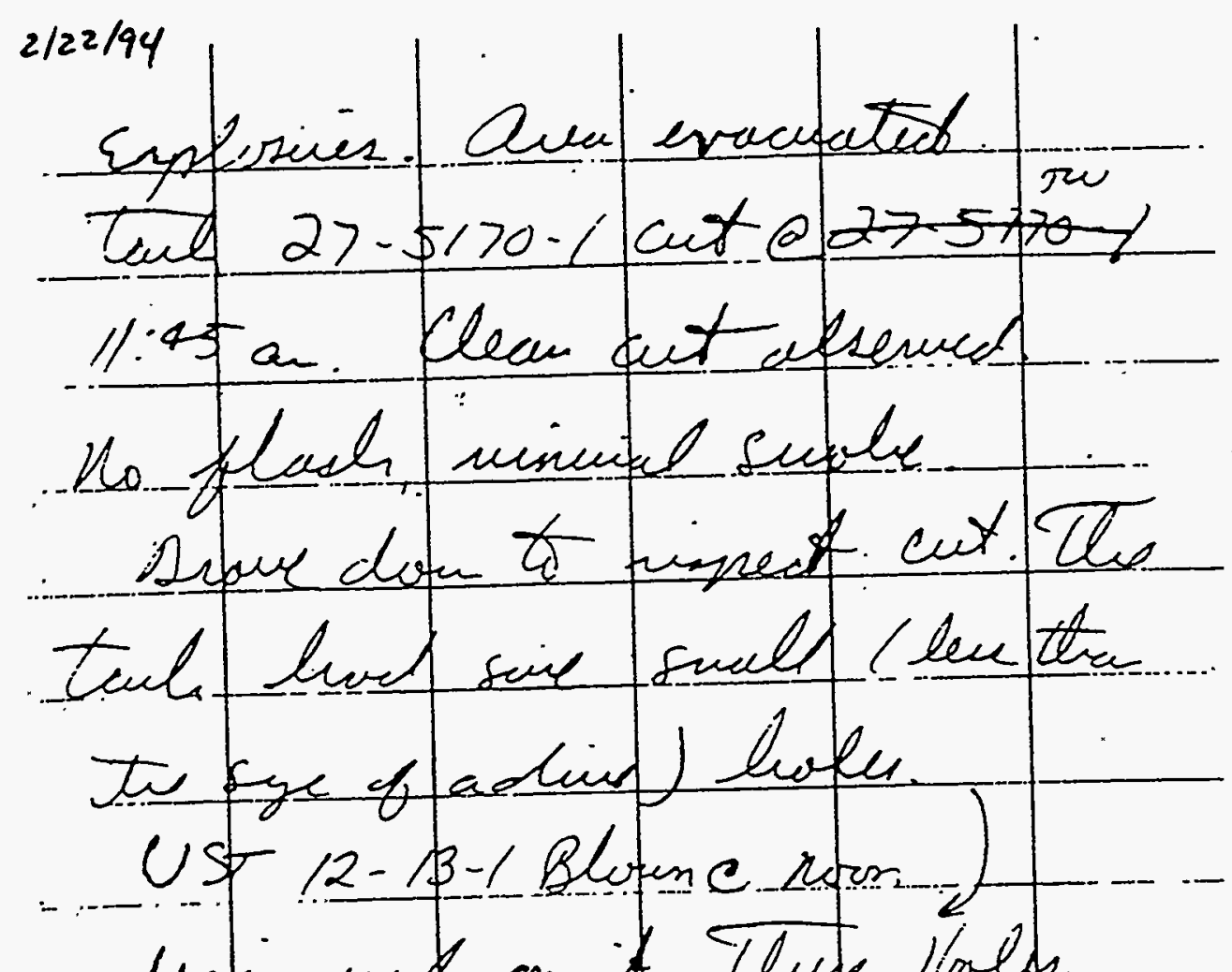
disponeref on it The lools uner not alsenes lefpre th shoot hut b wer filown any when the fed uns slut of

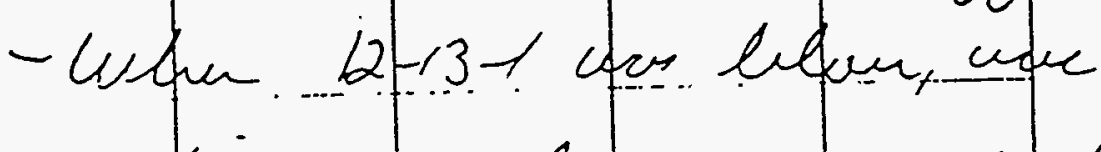
a trieg explofen sone stabe. 1) tow The was up sistaned

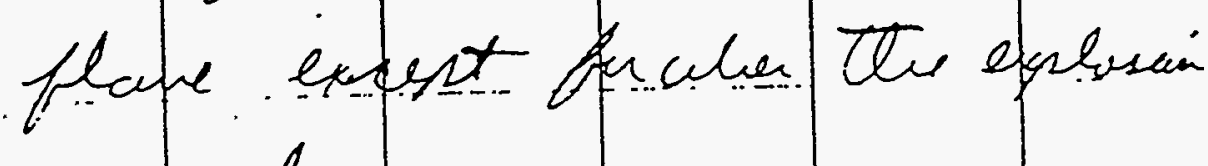
accued. 


\section{Reynolds Electrical \& Engineering Co., Inc.}

\section{MEMORANDUM}

To T. A. Wilczek THRU W. G. Flangas THRU

From R. J. Miller Rfur

Date $\quad$ February 7, 1994

Subject WORK REQUEST UPDATE

FOD-NTS Construction has completed the work you requested in your memorandum dated January 18, 1994. We provide the following details.

1. Area 27 - 5170 pi7e, Brady Number 592-050, to Area 6 hydrocarbon 1 andfil1 Completed January 26, 1994

2. Area 25 - 3205 pile, Brady Number 592-054, to Area 6 hydrocarbon 1 andfil1 Completed January 27, 1994

3. Area 12 - 8 pile, Brady Number 592-061, to Area 6 hydrocarbon 1 andfill Completed January 27, 1994

4. Area 12 - 13 pile, Brady Number 592-057, to Area 6 hydrocarbon 1 andfill Completed January 31,1994

5. Area 23 - 110 pile Brady Number 592-001, to Area 6 hydrocarbon 1 andfil1 Completed January 18, 1994

6. Area 23 - 112 pile, Brady Number 592-003, to Area 231 andfi 17 storage Completed February 2, 1994

If you have any questions please call me at 5-3926.

RJM:pjh0207-94

cy: Central Files 
From Page No.-

$=0.5$

...

Petoros.

v...unger

tordu. .

Arthe

rec.........

.1/6ppos. D. S. S.B.So

$1,2=7.9 .4$.

.

4heuso.

pore

. 1 thm

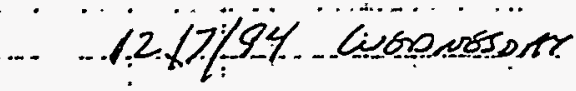

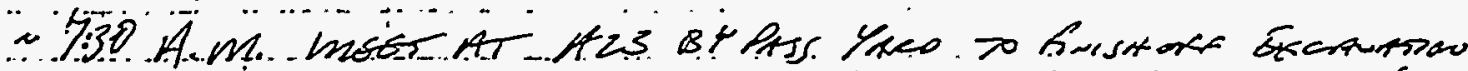

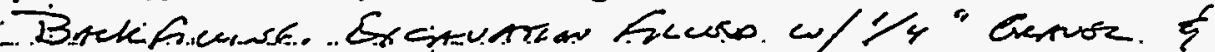

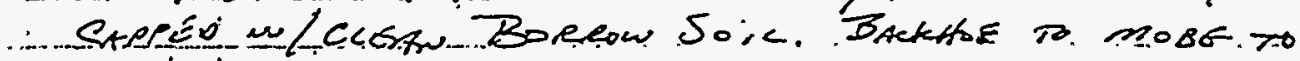

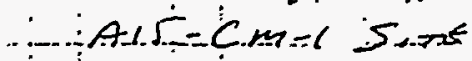

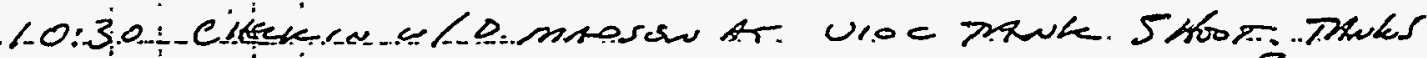

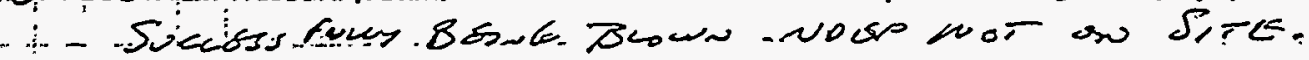

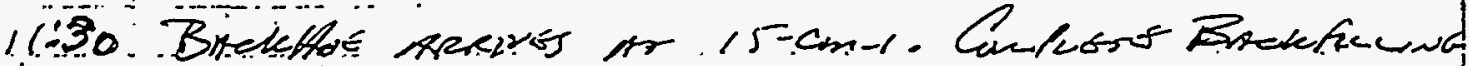

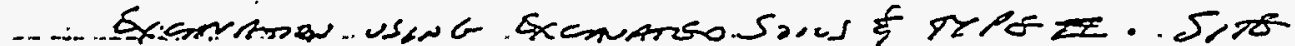

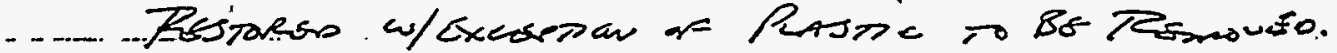
… -

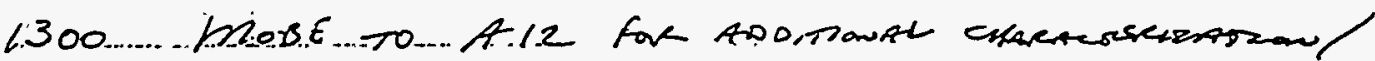
..... Rsmsorrson as . UST $/ 2-13-1$.

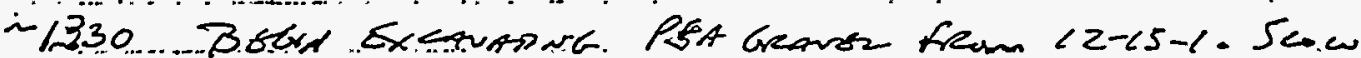

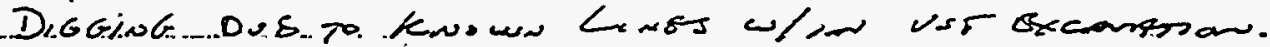

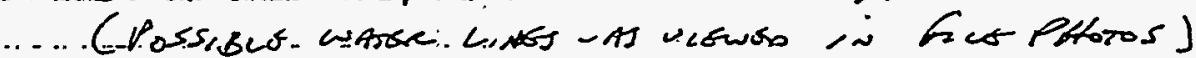

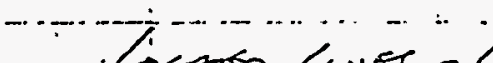

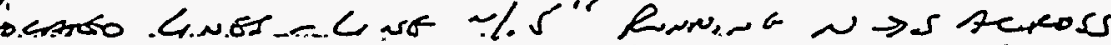

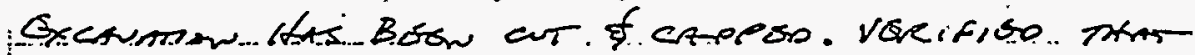

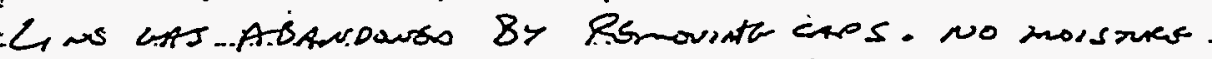

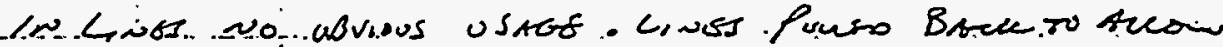

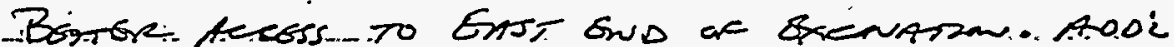

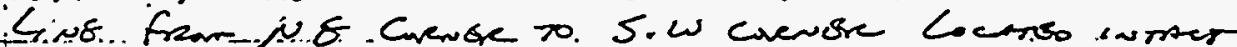

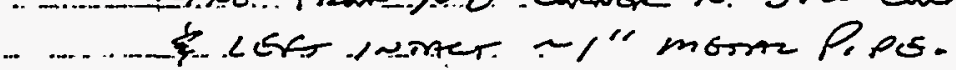

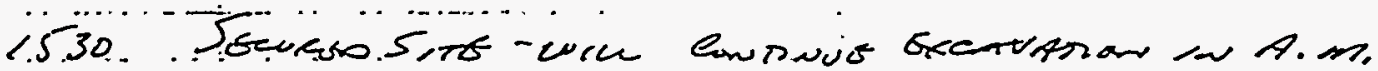

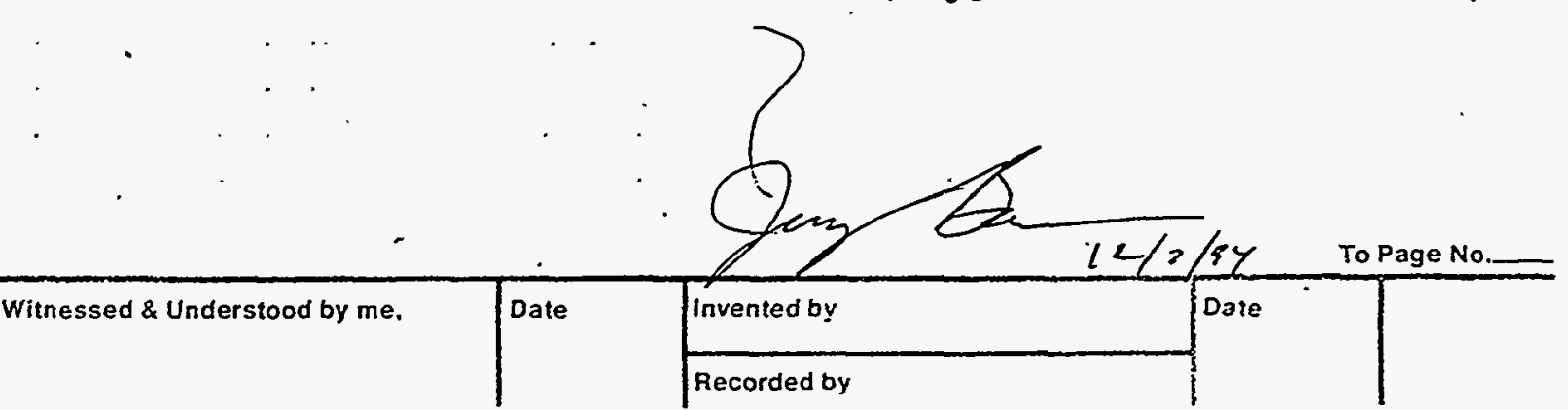


gato

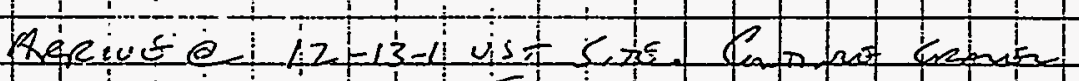
- exestan cesarat.

1k: 30

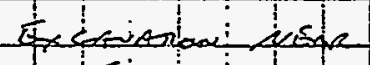

co socition Lis anisso

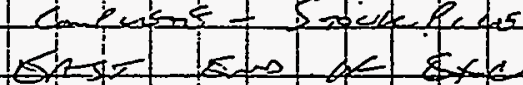

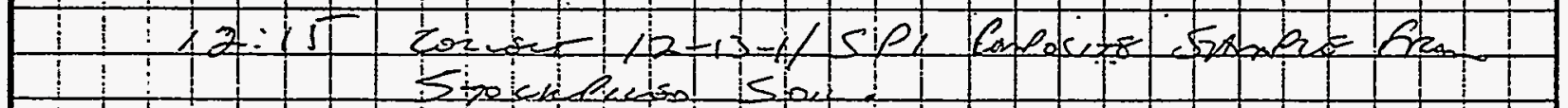

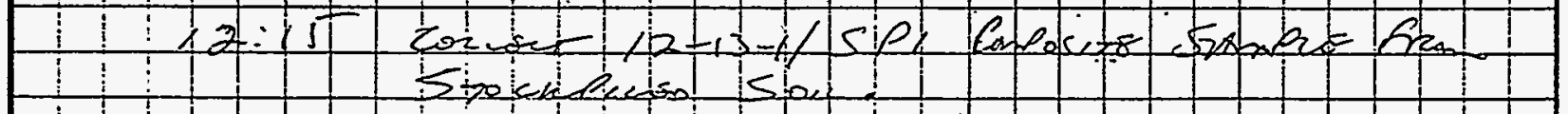

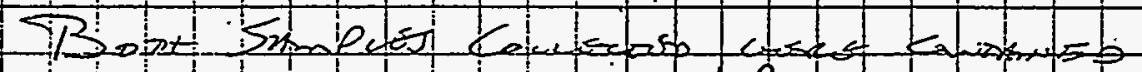

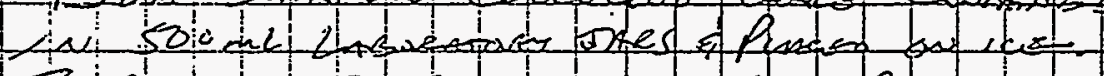

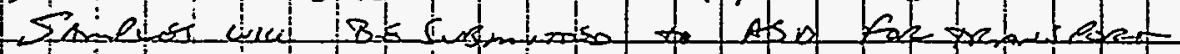
ti tos cks

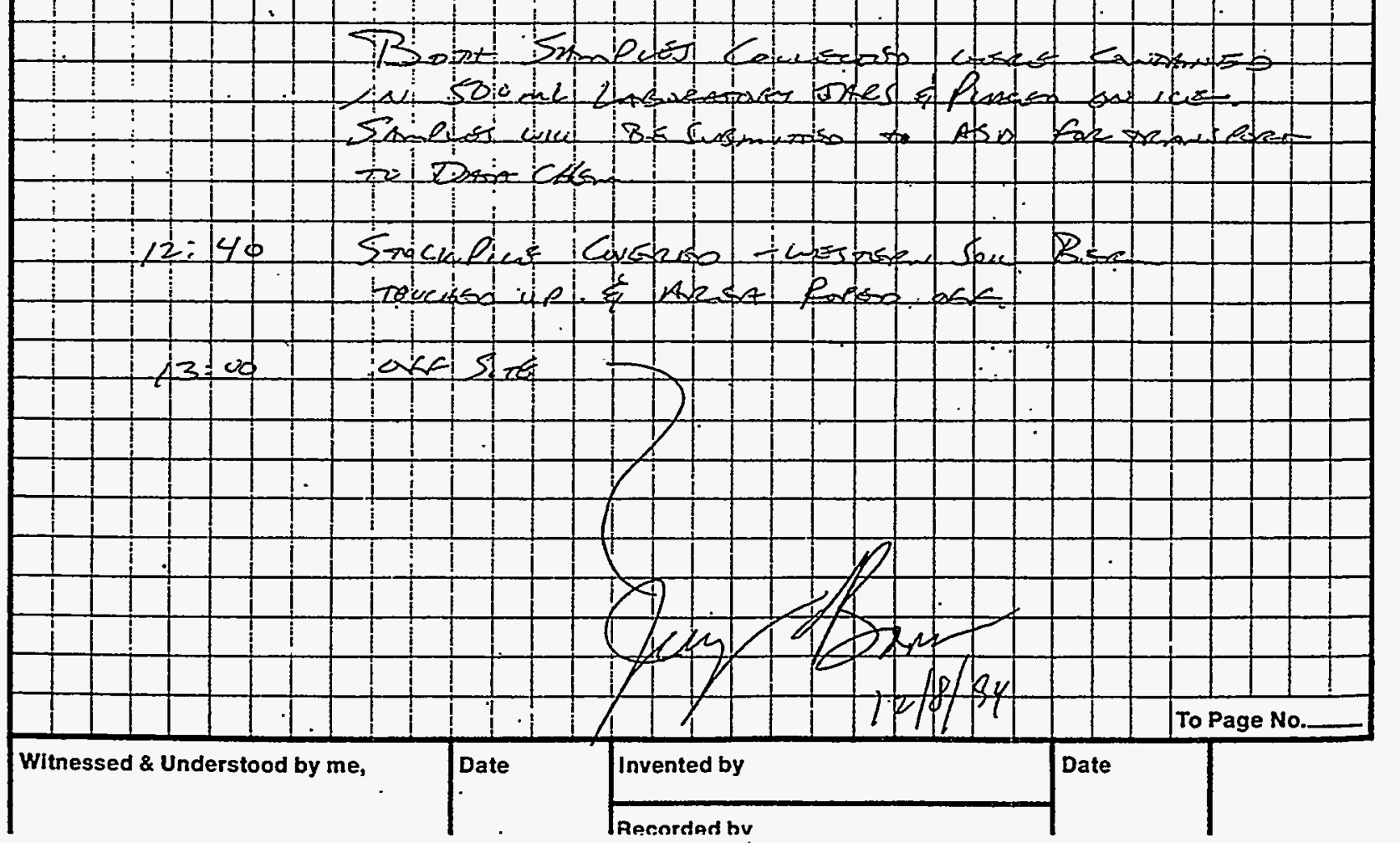




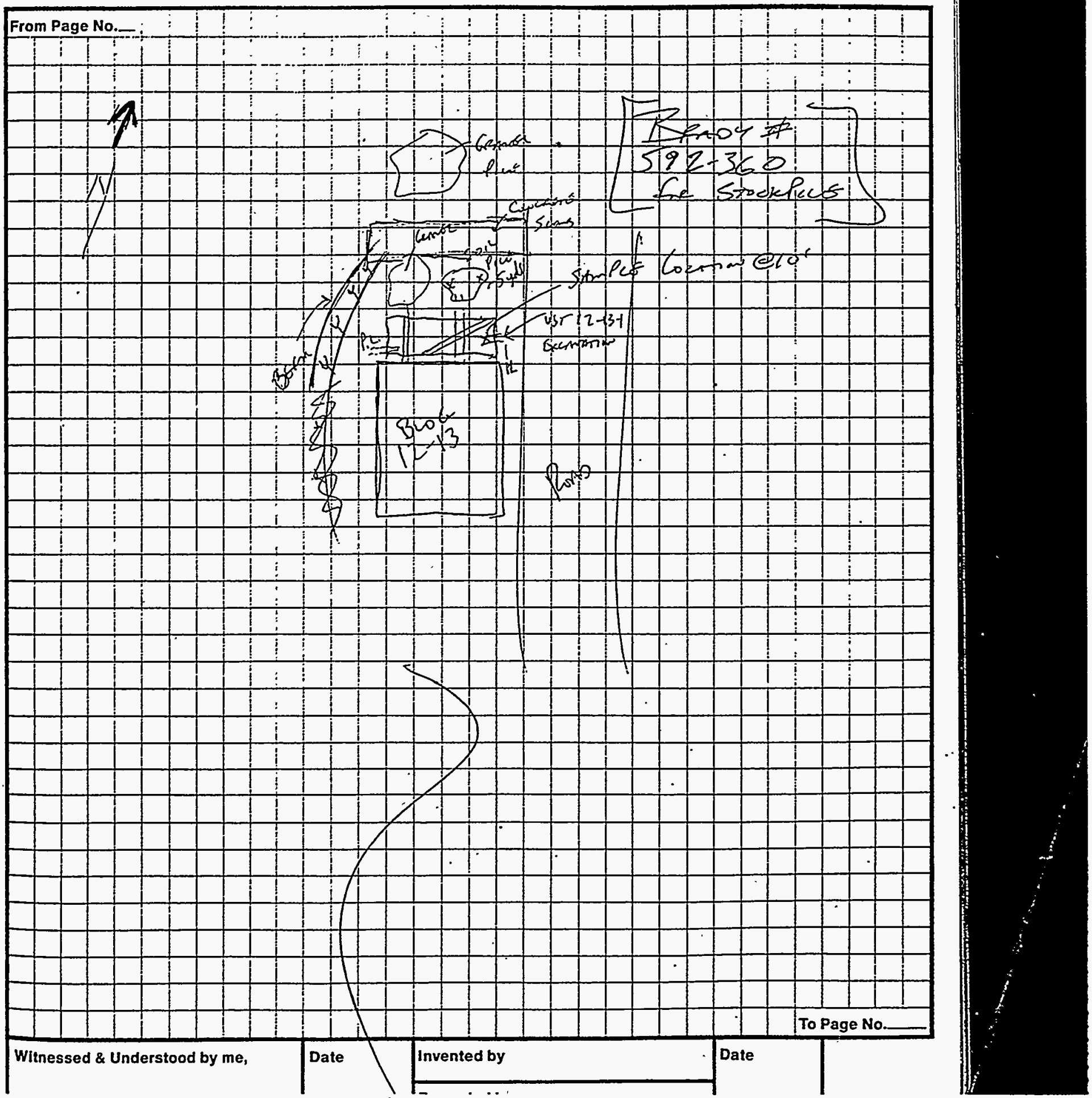


Project no.

Book No.

11

:rom Page No.

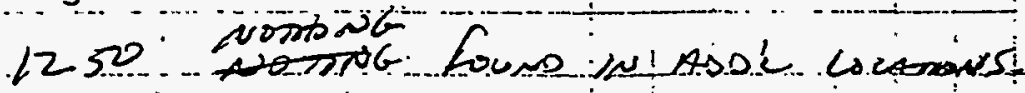

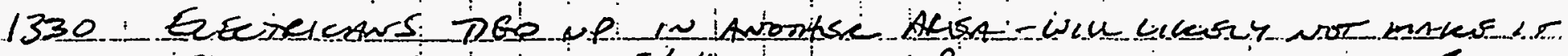

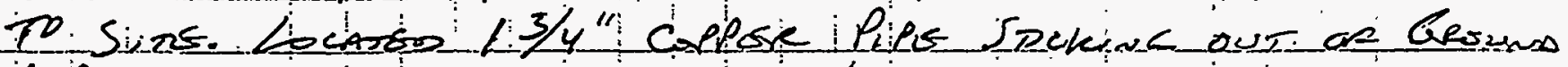

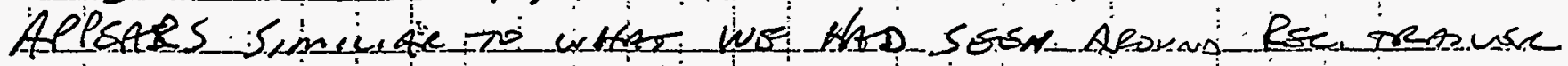

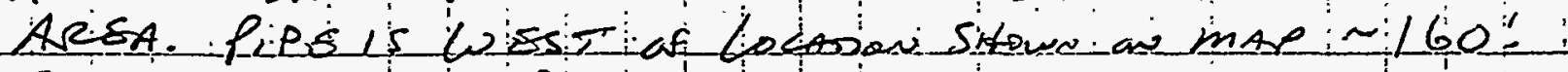

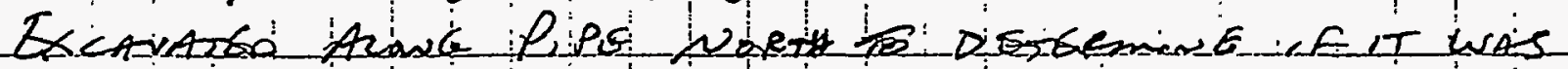

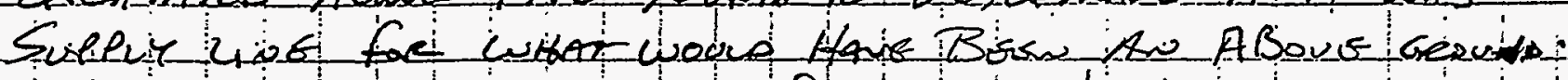

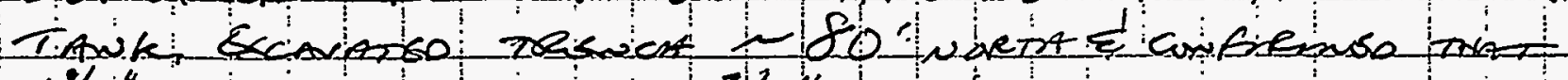

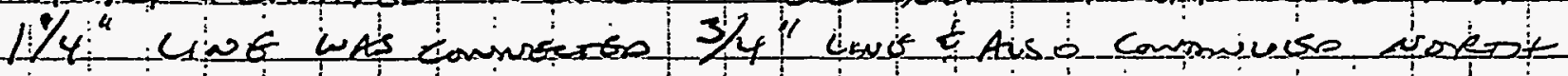

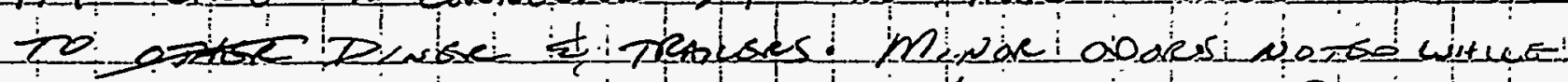

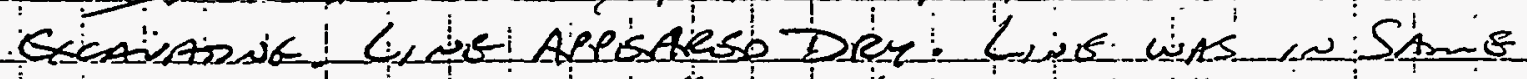

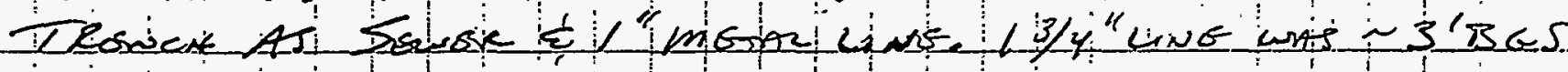

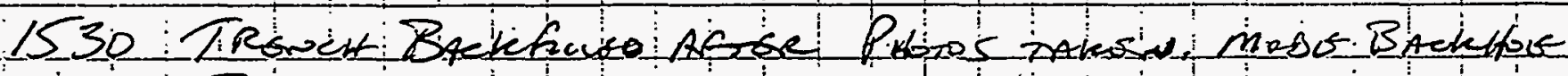

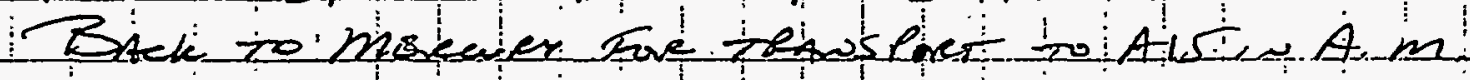

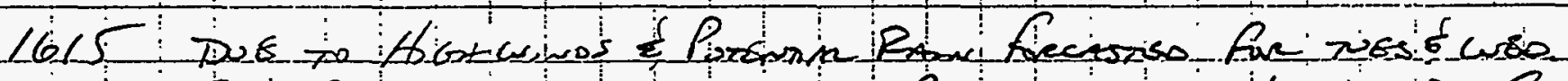

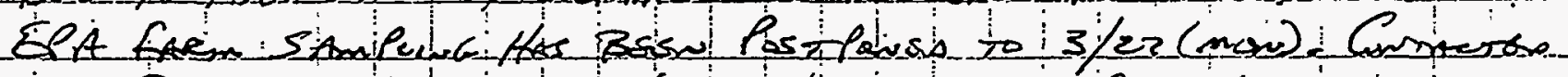

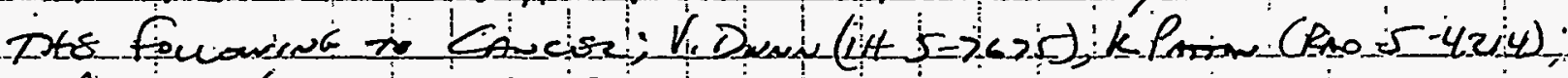

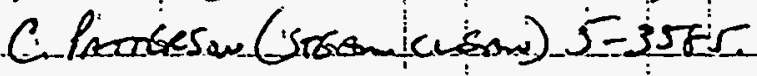

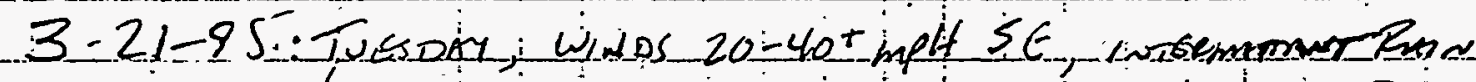

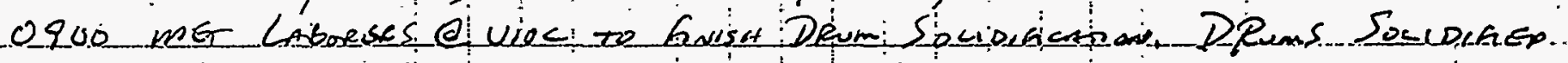

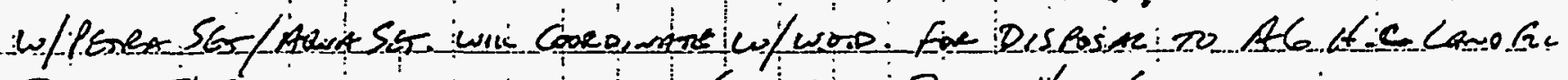

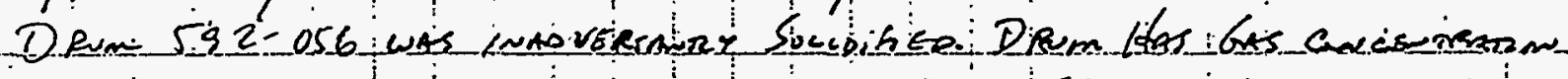

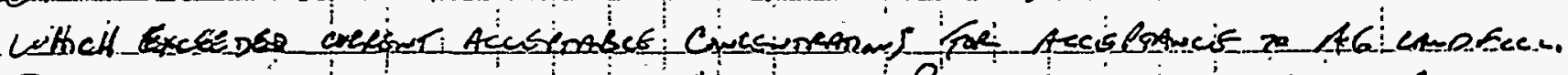

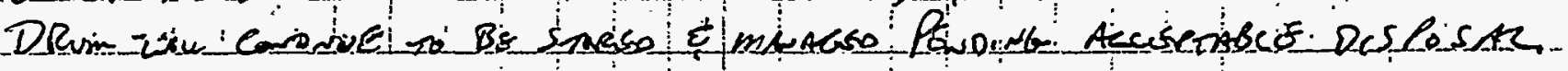

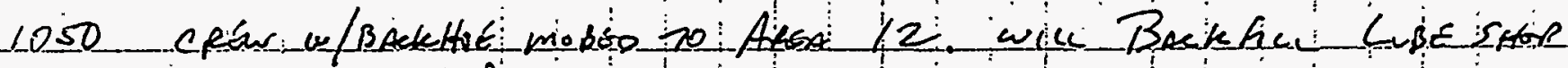

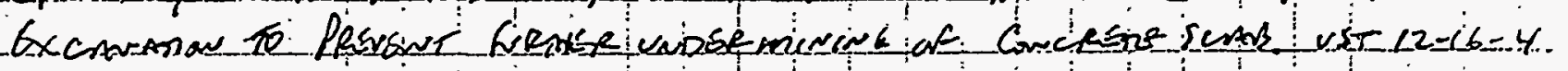

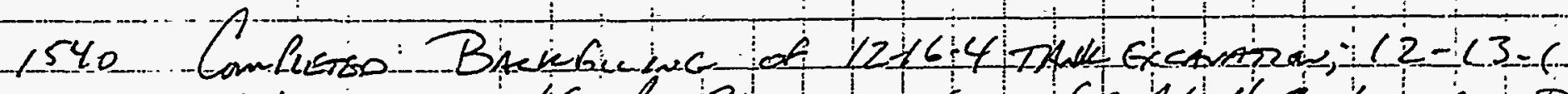

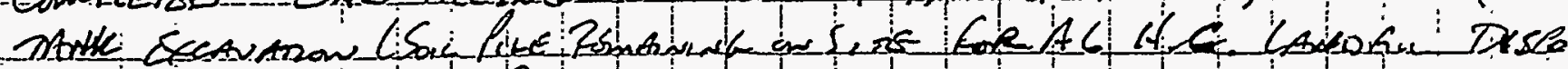

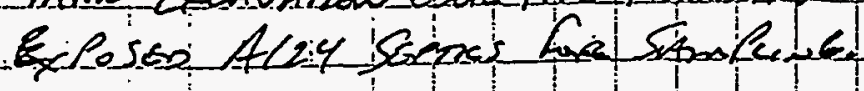

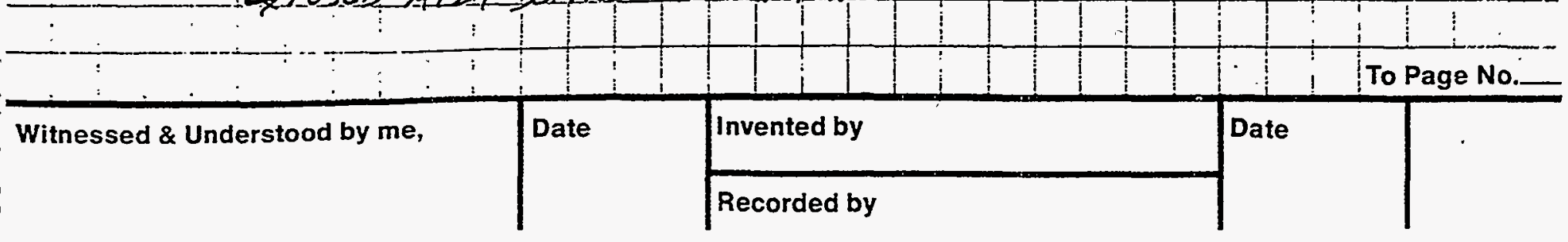


50 The

PROJECT NO.

Work continued from Page -

BOOK NO.

Work Order $(1090-143)$

Tuesday, 5-9-95

0260 Heading to Area 12 . Will remove soil piles from the Gas Station, tank 12-26-1,2,3 \& from Pow House, tank 12-13-1. Both piles will be transported to the Area 6 Hydrocarbon Landfill. 0815 Arrive at Gas Station willis: -Mike Powers $0830-2$ Teamsters arrive - Gene Noyes + Bud Sunnier (laborers) arrive. Equipment operator is over at Batch Plant and will be over as soon as possible. (John Dudley)

There are 3 piles located east of the gas station.

The northern most pie is to be removed today.

The others can $g$ back into the excavation.

0910 Begin Load \#1 - Brady Label \#592-212 (Note: this \# applies to all 3 piles.)

$15 \quad$ load $\$ 2$ filled

023s Waiting for Rad Sate so they can issue green tags.

Hath operator begin backfilling excavation with pea grave f

Sharon w rom Rad Safe arrives. Negative results. Issues

20 green tags

Load \#1 Tag A1970 Brady 592-212

Load $\#_{2}$ Tag A $A 1971$

1000 Loads leave. Want over to the Power House with Sharon to scan that pile. Issue tags.

${ }^{25}$.110 Trucks return. Begin loading

$\begin{array}{ccc}\text { Load } \# 3 & \text { Tag \#A1972 } & \text { Brady } 592-212 \\ \text { Load } \# 4 & \operatorname{Tag}^{*} A 1973 & \end{array}$

1130 Trucks leave. Resume backfilling

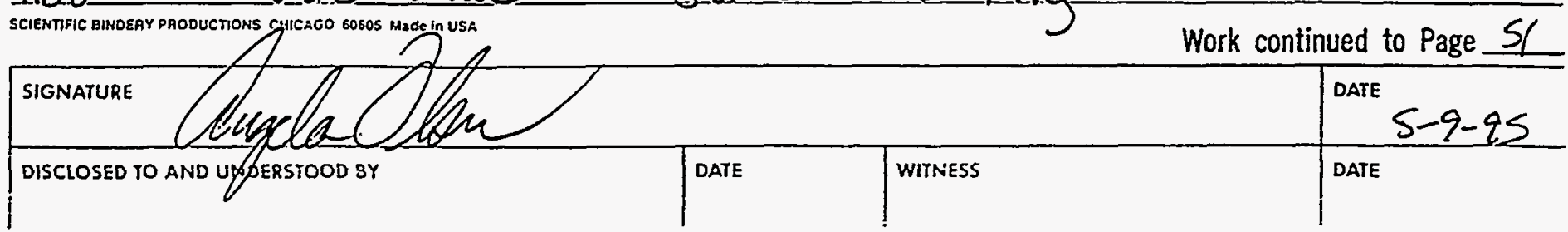


PROJECT NO.

51

continued from Page 50

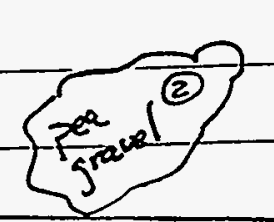

BOOK NO.

Reaver Mesa Rd

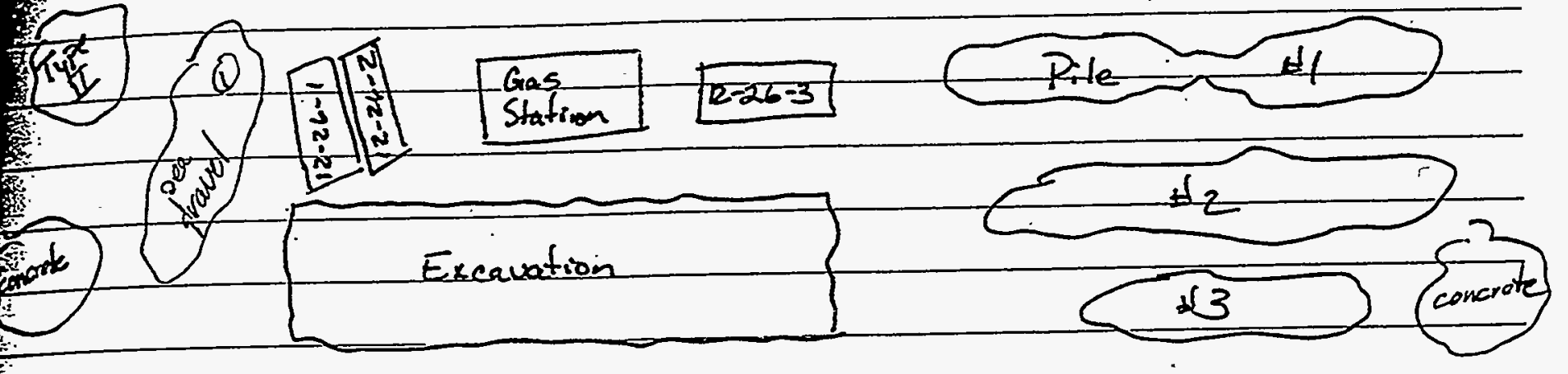

Pile $\# 1 \Rightarrow$ Area 6 Hydrocarbon Landfill

1150 Lunch

1220 Resume backfilling

5. $1310^{\circ}$ Trucks arrive

Load \$5 Green Tag \#A1974 Brady \#592-212

Lad ${ }^{*} 6$ Green Tag \#A1969. Brady $\frac{\$ 592-212}{592-360}$

Each truck has capacity of 24 cubic yards.

Total from pile \#1 i 130 af $(592-212)$

Total from Power House $\sim 5$ dy $(592-360)$

1430 Complete loading of hydrocarbon impacted soils

John has completed the removal of the 2 pea gravel piles. It filled the excavation to

5. approximately 4 feet from the top. Continued backfilling with pile $\# 3$

1440 Spoke with Pave Lory $(\times 3761)$ to schedule 1 truck to fill remove soils that wont fit in excavation

Work continued to Page 52 
52 TITLE

PROJECT NO.

Work continued from Page 5

BOOK NO.

o a scrap truck to haul off concrete. Need to call

him in the Am. to verify

1530 Mike returns. Fill one load from pile th to go to 10 c landfill

John completed removal of pile \#3+part of pile 2 and placed in excavation. Compacted as he went. Bud + Gene collected plastic from the piles that had blown off - very broken up + disintegrated. The drums were also scattered around. Collected $t$

10 placed on pallets.

1600 Head back to Mercury. Met Willie, the other driver, on the way out. He was bringing out a load of Type II soil for the top one foot of fill.

15

Wednesday 5-10-95

ono Called Dave Lory. the's got a scrap dump headed out to Area 12 Gas Station to pick up the concrete. Try shave a dump for soil before noon.

${ }^{20} 0230$ Head to Area 12

0820 Met with Bud Gene. John Dudley will load concrete. 0900 Load of concrete to to c landfill. Continue back filling
excavation from the remainder of pile th

${ }^{25} 1000$ Truck back-load more concrete

1020 Concrete to $10 \mathrm{i}$. Continue backfilling. Cancelled. other truck. Will be able to put rest of pile in excavation.

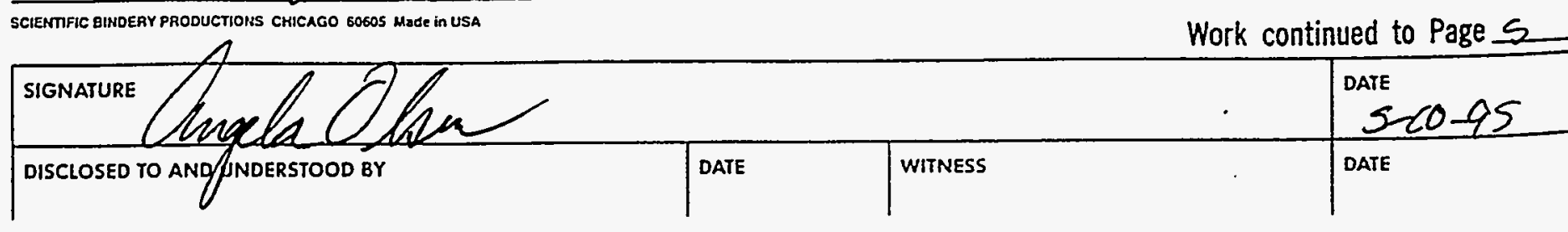


DRUM/BUCKET/PILE HISTORY REPORT

DRUM - INFORMATION AS OF:

DRUM NUMBER: $592-360$ SIZE IN GALLONS:

Drum_cotor:

Contents:

Label Type:
Diesel impletion. Sol Gran Rsinsoir

2

$5+1$

Excavation of $12-13-1$ U St

LOCATED AT: $\mathbb{A} / 2$

SAMPLE INFORMATION

REQUEST DATE:

DATE SAMPLED:

SAMPLE NUMBER:

SAMPLE TAKEN BY:

RESULTS RECEIVED:

SAMPLE RESULTS:

)

SIGNED BY:

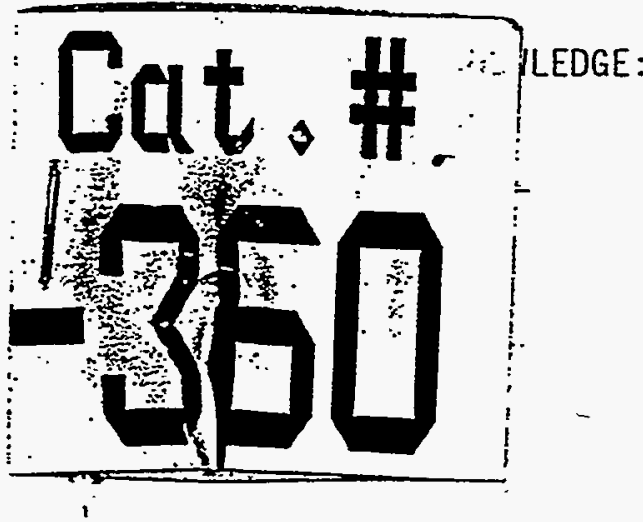

(List all information known, including origin).

DISPOSITION INFORMATION-

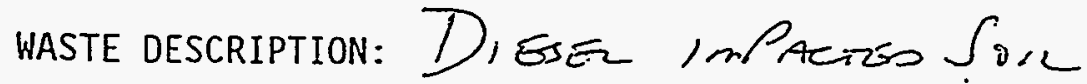

WOO CALLED:

Who_called: Rob Bostzecteo

Picked_up: $5 / 9 / 95$

SIGNED

HYDRocarbon Lanofic

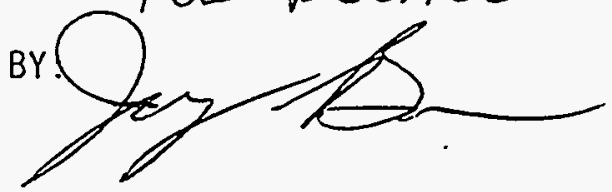




\title{
APPENDIX E
}

\section{SPILL NOTIFICATION}

\author{
REPORT
}





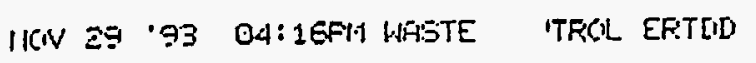

\section{DOE Novzda Fiold OHlos

- section A, L, and $M$ aro to do compleled by DOENV.

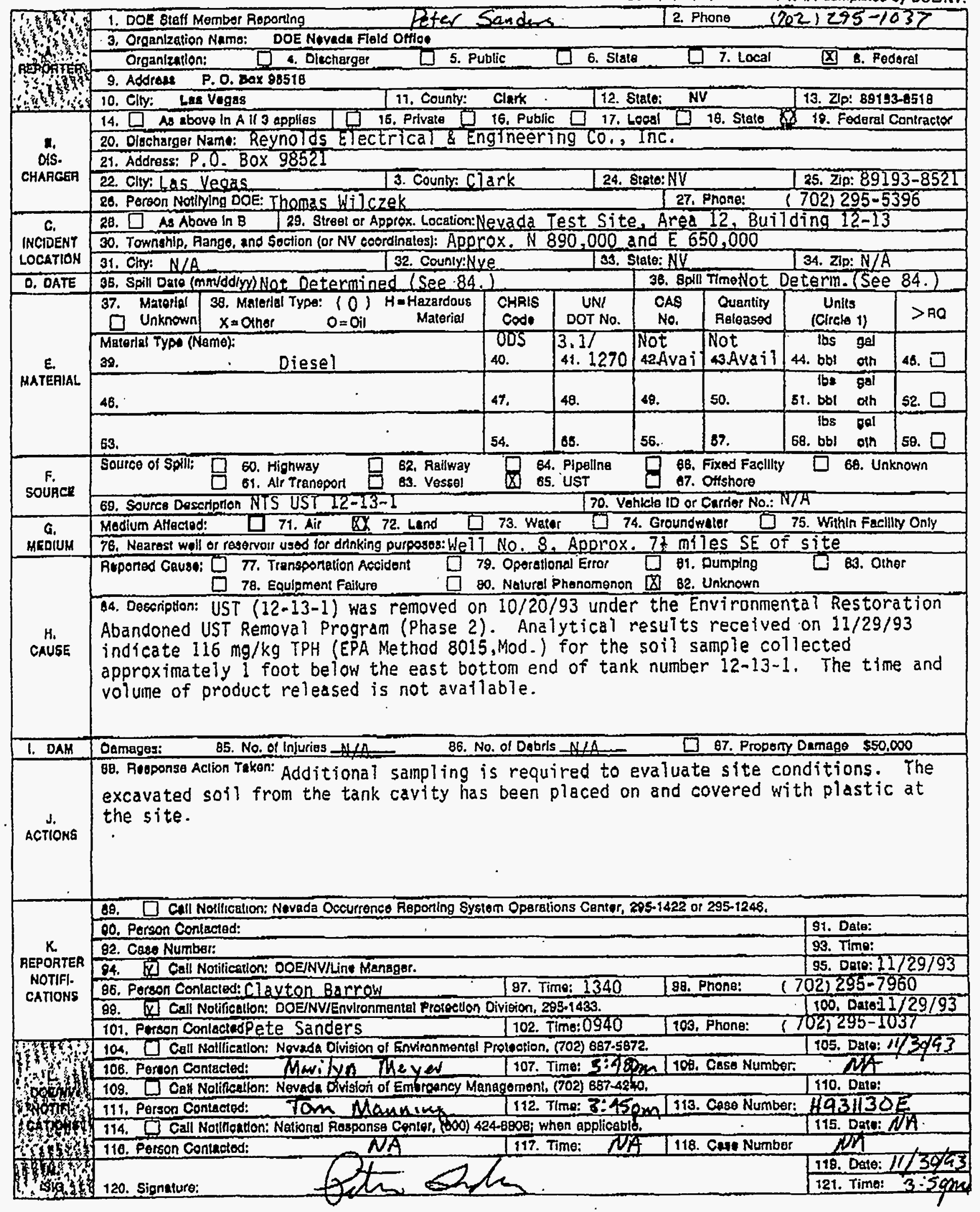





\section{APPENDIX F}

\section{LANDFILL DISPOSAL DOCUMENTATION}




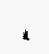



This vis is an acknowledgment that a Bill of Lading has been issued and is not the Original
Bill of Lading, nor a copy or duplicate, covering the property named herein, and is

Shipper No. $539-41 ?-2$

Carrier No.

Page 1 of 1

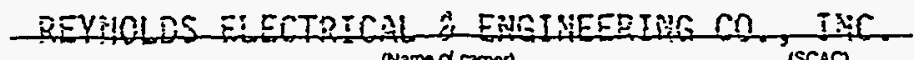

Date $01-27-96$

TO: LASTE OPERATIOUIS, ÄTT: K. A. HGGAR

\section{Consignee}

On Collect on Delivery shipments, the letters "COO" must appear belore consignee's name

or as otherwise provided in liem 430 . Sec. 1 .

Streot

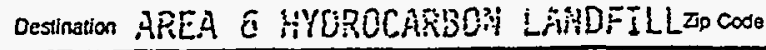
(Name o carron)

$(\mathrm{SCAC})$

\author{
FROM: R. J. HILLER \\ Shipper FOO - HTS COASTRUCTION
}

Street

Orgin AEEA 12 UST UPCRADE BIDGS. 12-3 \& 12-13

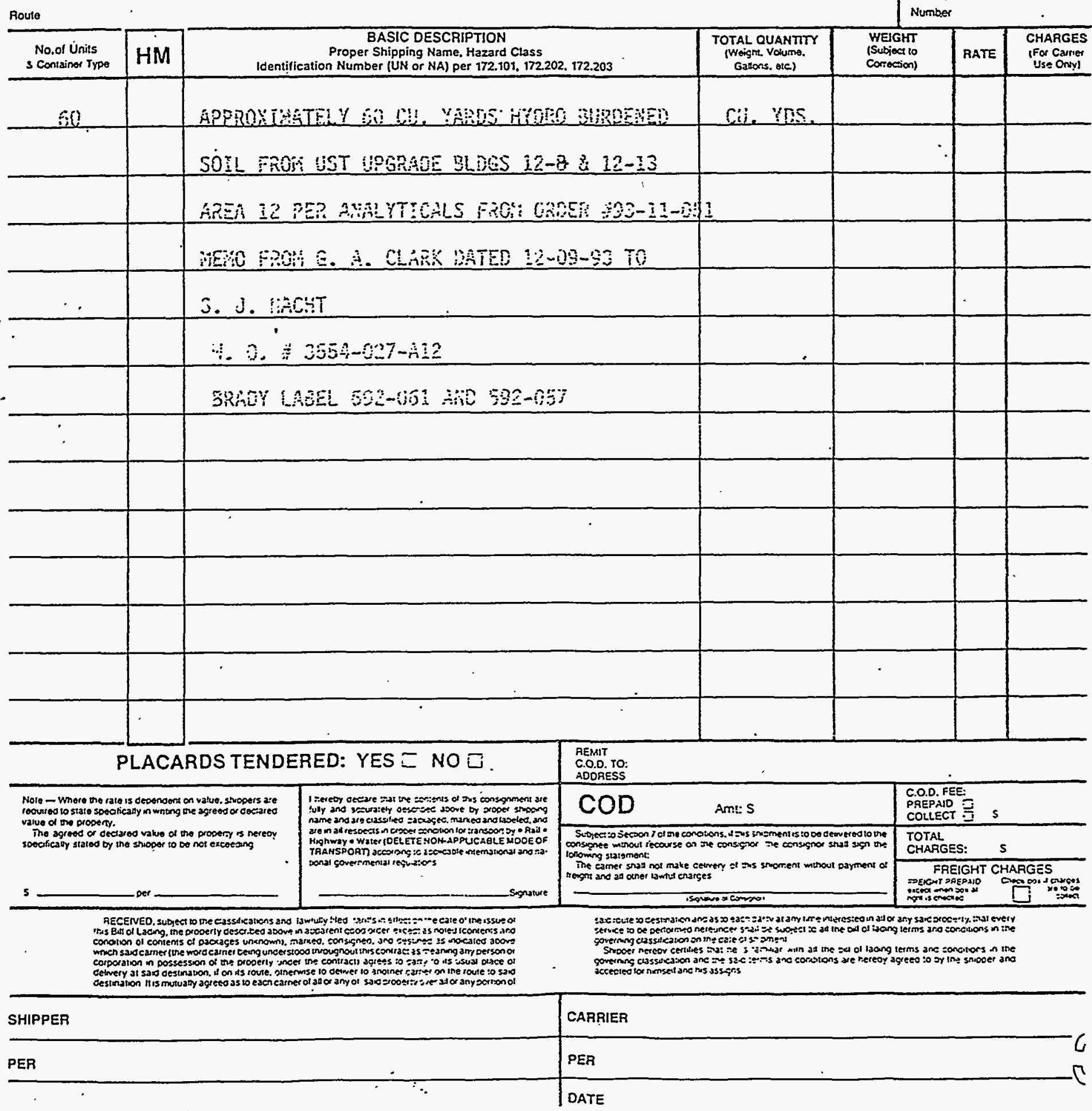




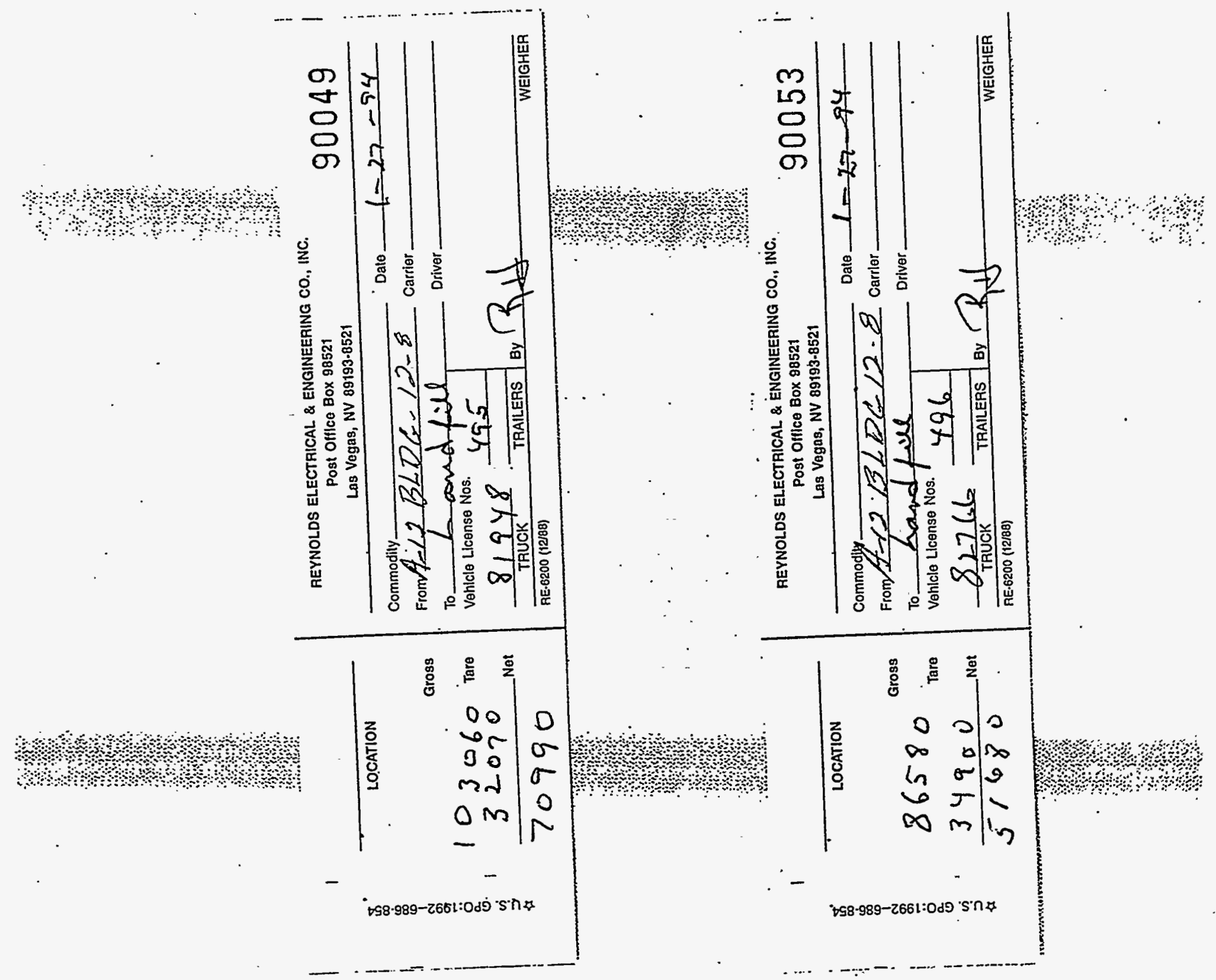



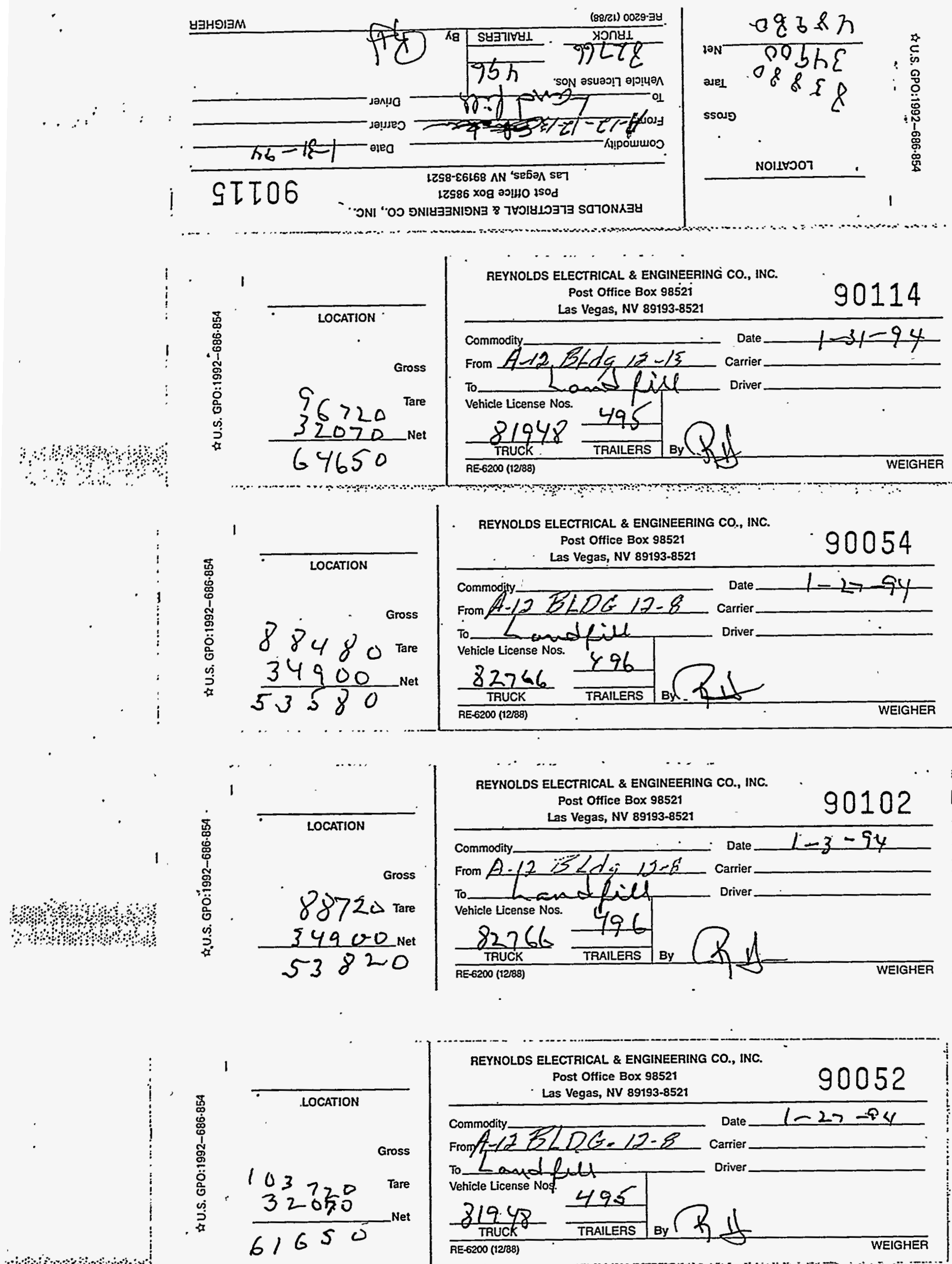

REYNOLDS ELECTRICAL \& ENGINEERING CO., INC. - Las Vegas, NV 89193-8521

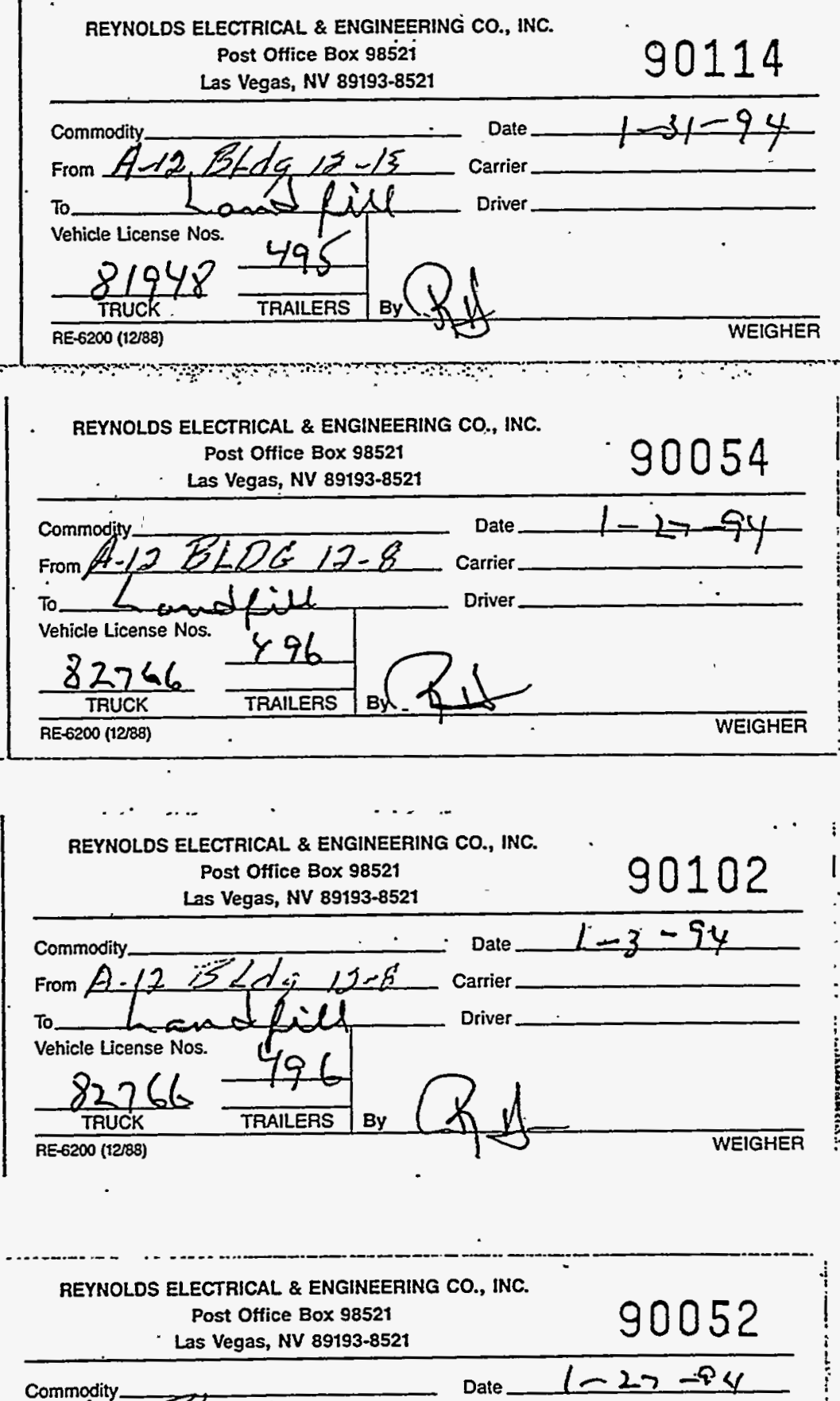




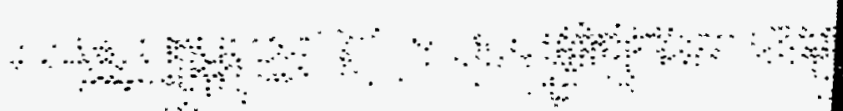

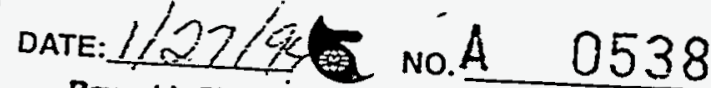

Reynolds Electrical \& Engineering Co., Inc.

Radiological Control Department-Ramatrol

Material Clearance

MONITOR: isirié

ORGANIZATION: $A \Rightarrow y^{2}$ Dococe.

SURVEY LOCATION:

TO

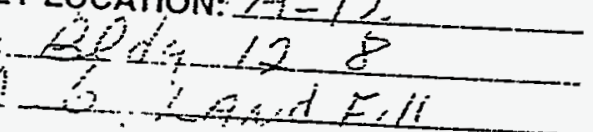

PUNRESTRICFED

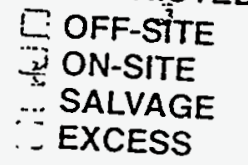

THIS MATERLAL HAS BEEN SURVEYED FÖR: INITIAL

RADIOACTIVTY ANO MEETS DOE

STANDARDS FOR RELEASE TO PUBLC USE

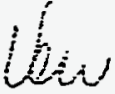

CONTROLLED

DESCRIPTION:

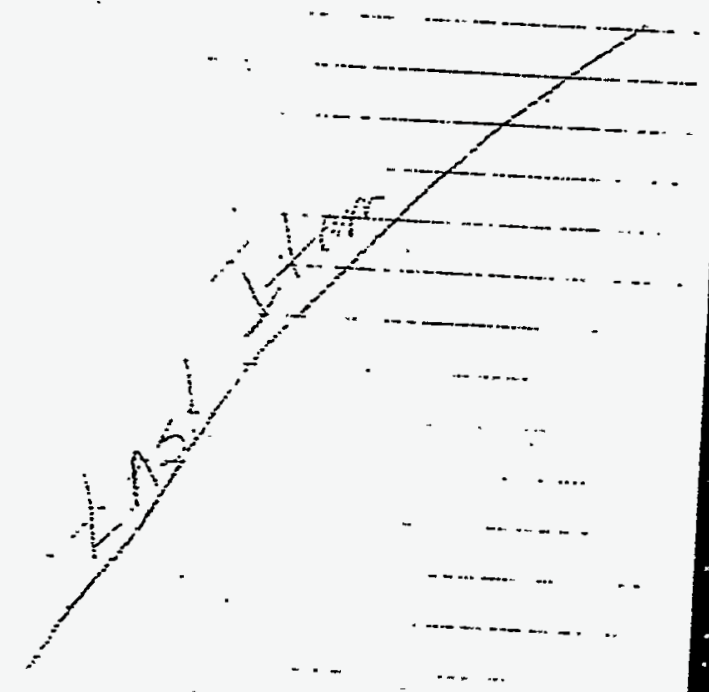

REMARKS:

$=$

$7 \frac{1}{9}=\frac{1}{4}$

RE-158) (06:93) 


\section{SUNRESTRLCTED \\ $\square$ OFF-SॉTE \\ 因 ON-SITE \\ SALVAGE \\ $\square$ EXCESS}

THIS MATERIAL HAS BEEN SURVEYED FOR RADIOACTIVTY AND MEETS DOE STANDARDS FOR RELEASE TO PUBLC USE

\section{$\square$ CONTROLLED}

DESCRIPTION: Lind o Dipt

low
INITIAL
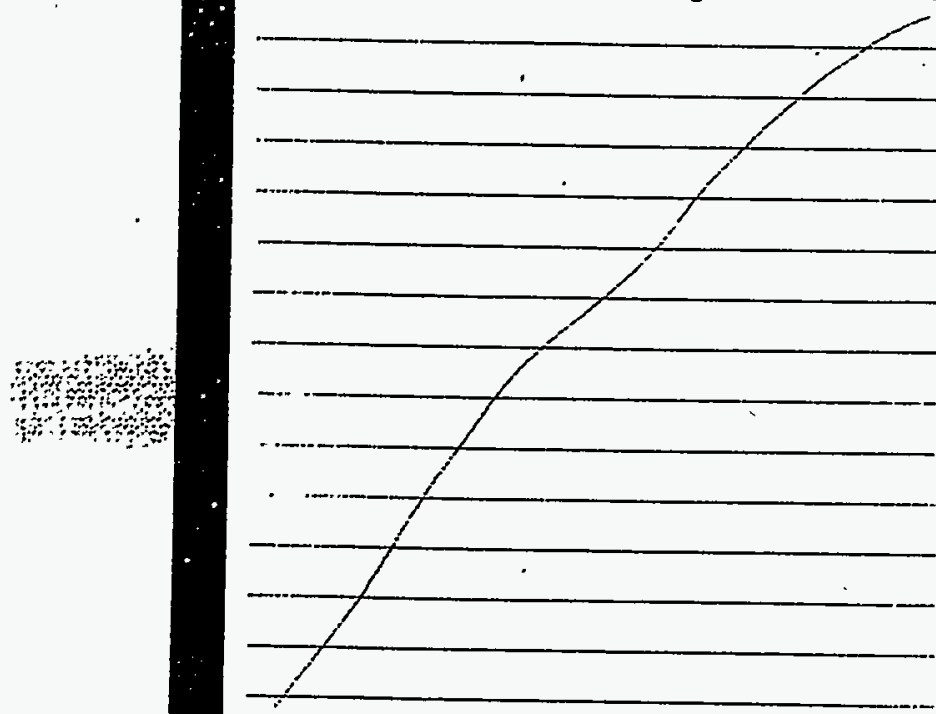

REMARKS:

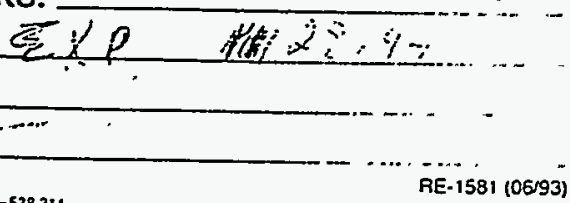

DATE:L/27/24 No.A 0536

Reynolds Electrical \& Engineering Co., Inc. Radiological Control Department-Ramatrol

\section{Material Clearance} MONITOR: WARRd organization: Dee = SURVEY LOCATION: $A-12$ FROM: $00 d a<2-13$ TO:

\section{UNRESTRICTED \\ $\square$ OFF-SITE \\ 18 ON-SITE \\ $\square$ SALVAGE \\ $\square$ EXCESS}

\section{THIS MATERLAL HAS BEEN SURVEYED FOR RADIOACTIVITY AND MEETS DOE STANDARDS FOR RELEASE TO PUBLLC USE

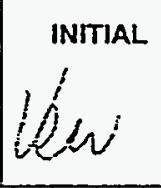

\section{ב CONTROLLED}

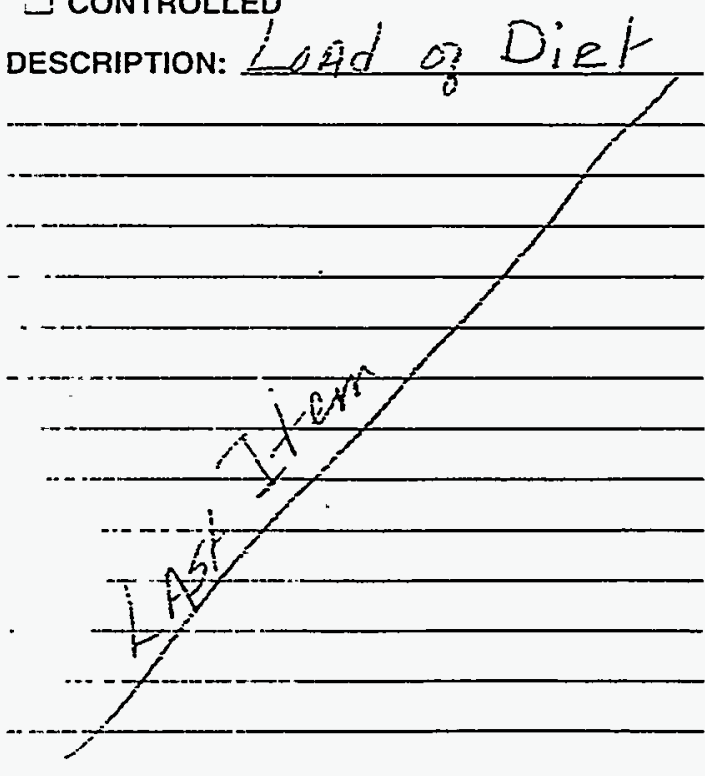

REMARKS:

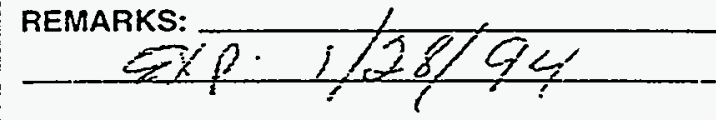


DATE: $/ 22744$ No.A 0537

Reynolds Electrical \& Engineering $\mathrm{Co}$. Inc. Radiological Control Department-Ramatrol

\section{Material Clearance}

MONITOR: LtiAf d

ORgaNIZATION: Boe-cw

SURVEY LOCATION: $A-12$

FROM: BSia 12 i

To: $1-6$ ind $f i 1$

LOUNRESTRLCTED
$\square$ OFF-SITE
D ON-SITE
$\square$ SALVAGE
$\square$ EXCESS

THIS MATERLAL HAS BEEN SURVEYED FOA RADHOACTIVTY AND MEETS DOE STANDARDS FOR RELEASE TO PUBLIC USE

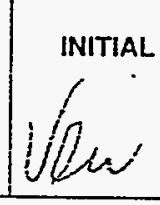

$\square$ CONTROLLED

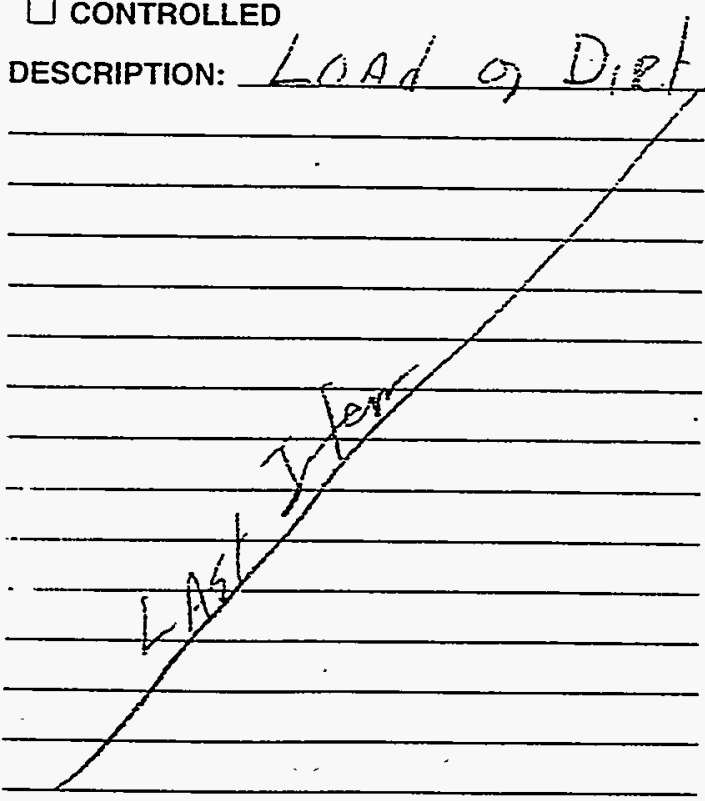

REMARKS:

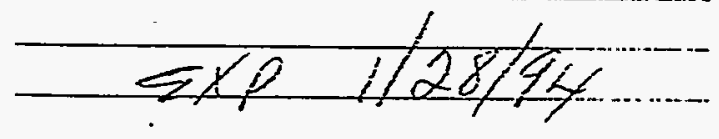

(3)

DATE: $/ / 27 / 94=$ No.A 0535

Reynolds Electrical \& Engineering $C_{0 .}$ inc. Radiological Control Department-Ramatrol

\section{Material Clearance} MONITOR: CᄉAREd

ORGANIZATION:

SURVEY LOCATION: $\theta-12$

FROM: $20 ; ; 2-13$

TO:

Andill

\section{ZIUNRESTRICTED}
$\square$ OFF-SITE
Q ON-SITE
$\square$ SALVAGE
DEXCESS

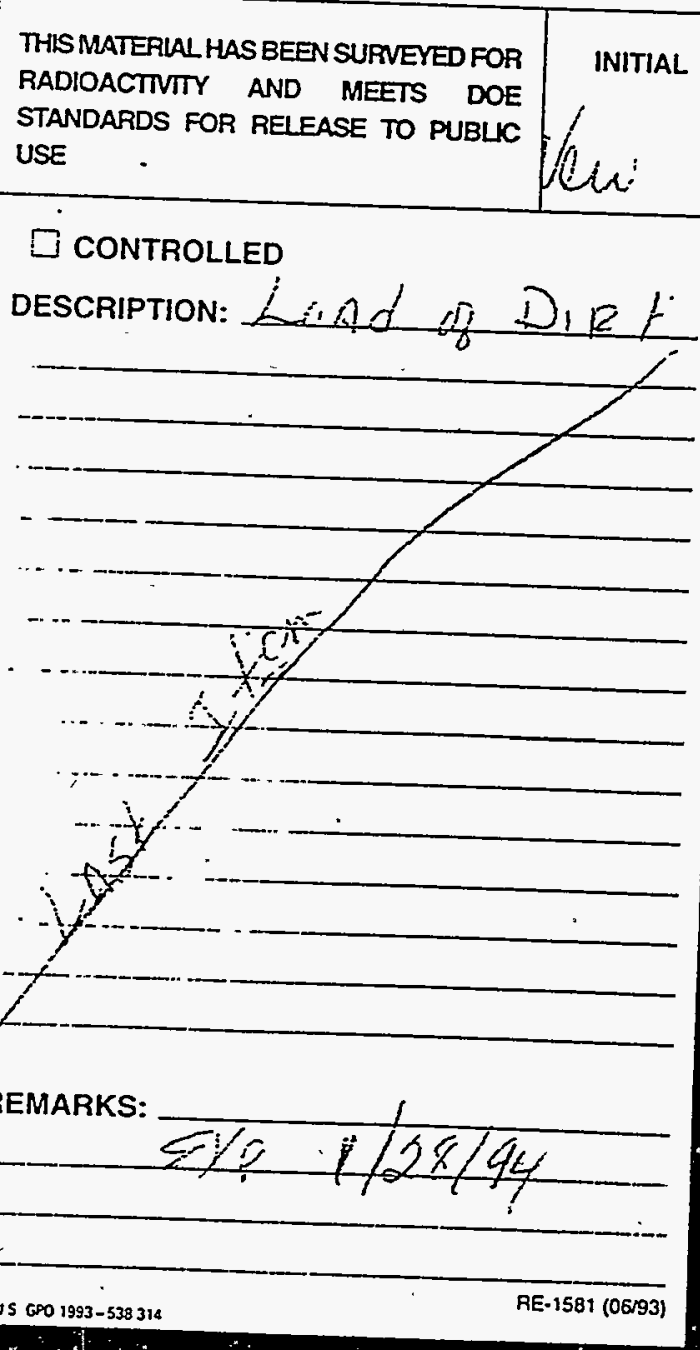

THIS MATERIAL HAS BEEN SURVEYED FOR RADIOACTIVTY AND MEETS DOE STANDARDS FOR RELEASE TO PUBUIC USE

\section{$\square$ CONTROLLED}

DESCRIPTION: LAGd of DIRf 
DATE: $/ / 27 / 44$ No.A 0539 Radiological Control Department-Ramatrol

\section{Material Clearance}

MONITOR: LA fed

ORganization: Ter.ce

SURVEY LOCATION: $A-12$ FROM: $60 d \quad 12<8$ TO:

\section{DUNRESTRICTED}
$\square$ OFF-SITE
COON-SITE
$\square$ SALVAGE
$\square$ EXCESS

THIS MATERLALHAS BEEN SURVEYED FOR RADIOACTIVITY AND MEETS DOE STANDARDS FOR RELEASE TO PUBLC USE
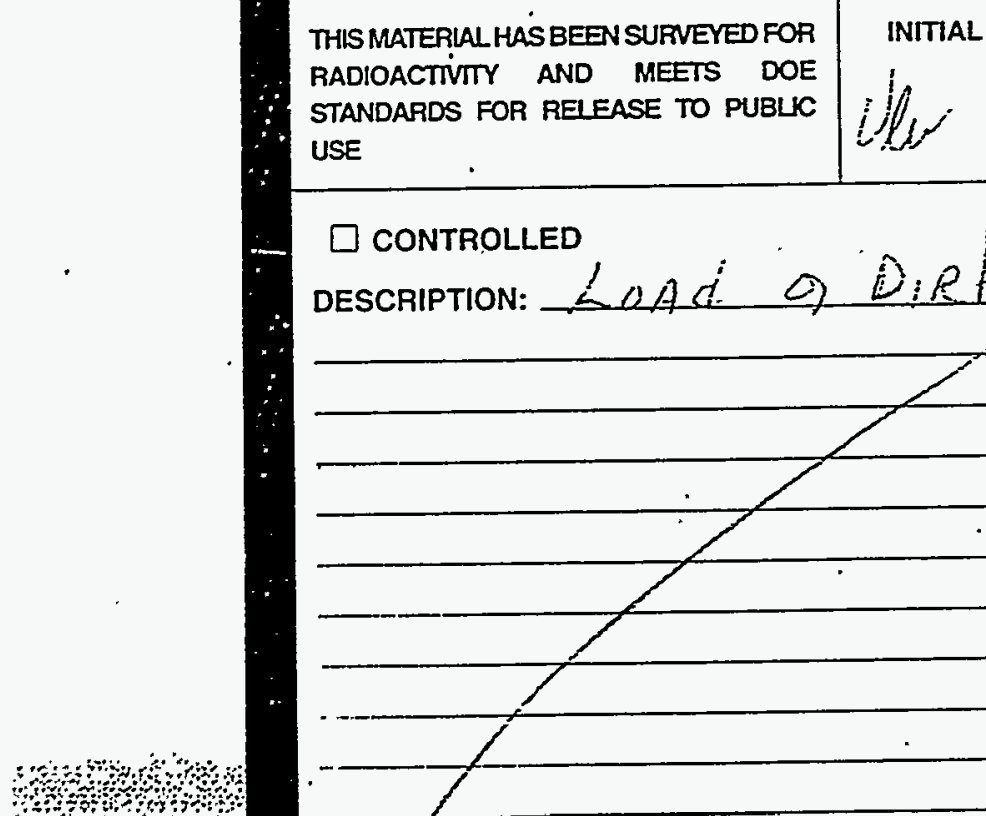

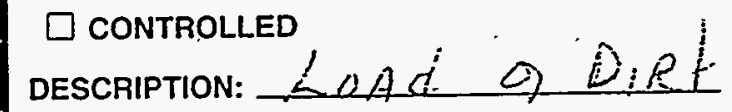

REMARKS:

$$
\text { Exp 1/29/94 }
$$

RE-1581 (06193)

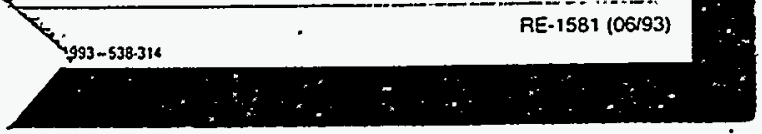

DATE: 1/27/94 No.A 0501

Reynolds Electrical \& Engineering Co., Inc. Radiological Control Department-Ramatrol

\section{Material Clearance} MONITOR: WAR C ORGANIZATION: Lieno SURVEY LOCATION: $\frac{1}{12-12}$ FROM: $3 i d i \frac{1}{12-8}$ TO

\section{母 UNRESTRICTED}
$\square$ OFF-SITE
DON-SITE
SALVAGE
EXCESS

THIS MATERLAL HAS BEEN SURVEYED FOR RADIOACTIVTY AND MEETS DOE STANDARDS FOR RELEASE TO PUBLLC USE INITIAL itis

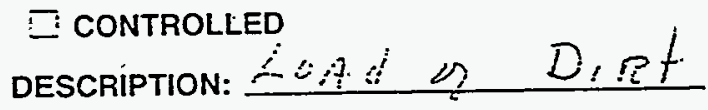

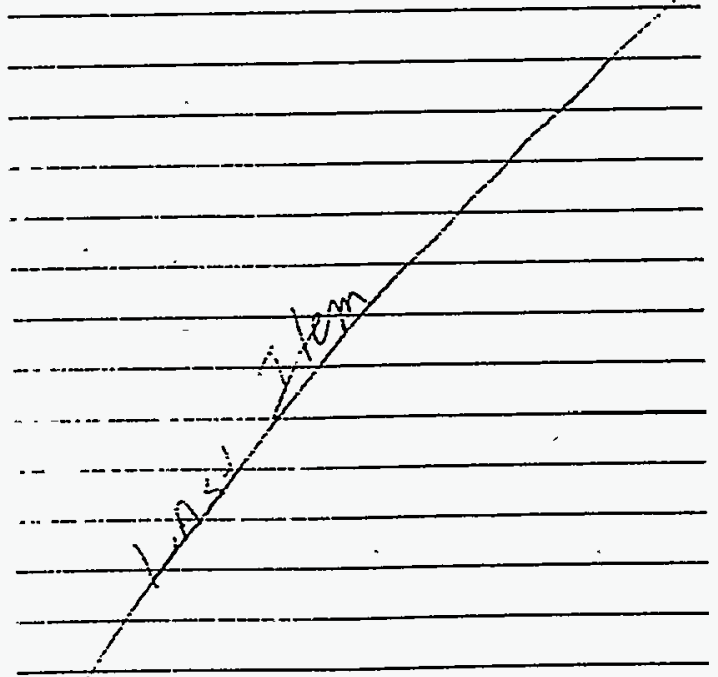

REMARKS: 


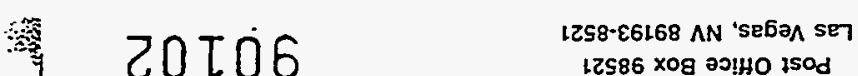

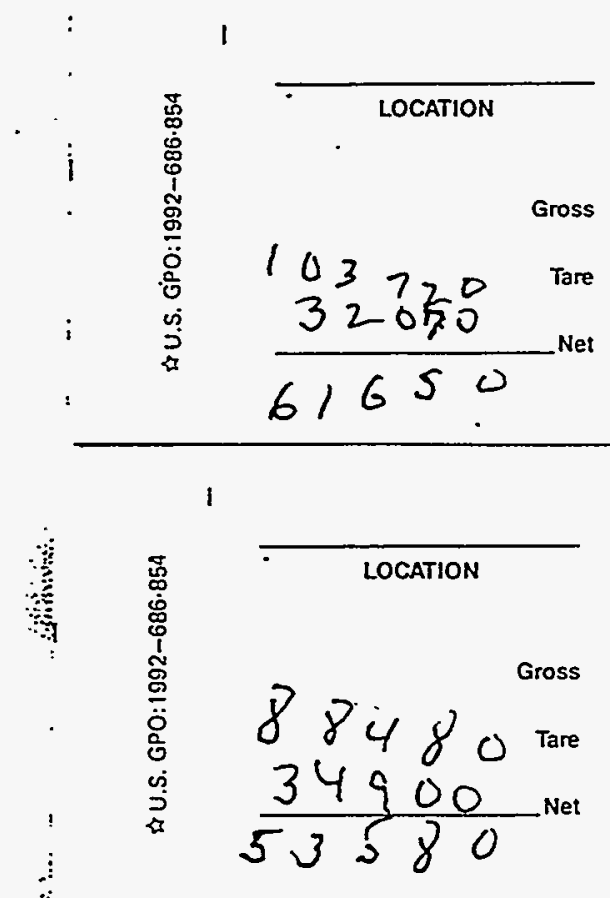

REYNOLOS ELECTRICAL \& ENGINEERING CO., INC.

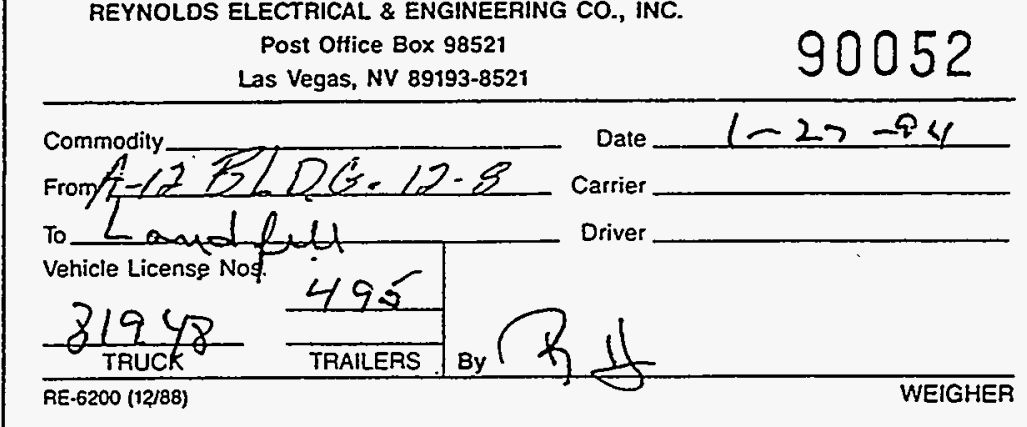

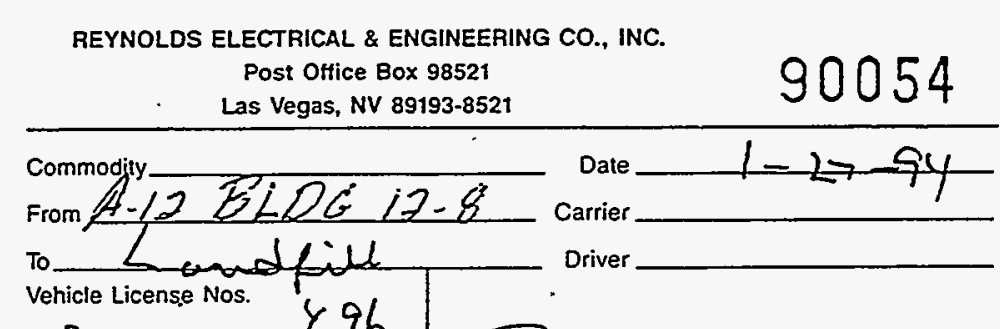

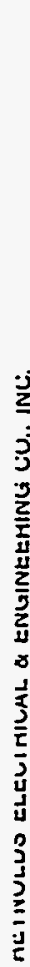
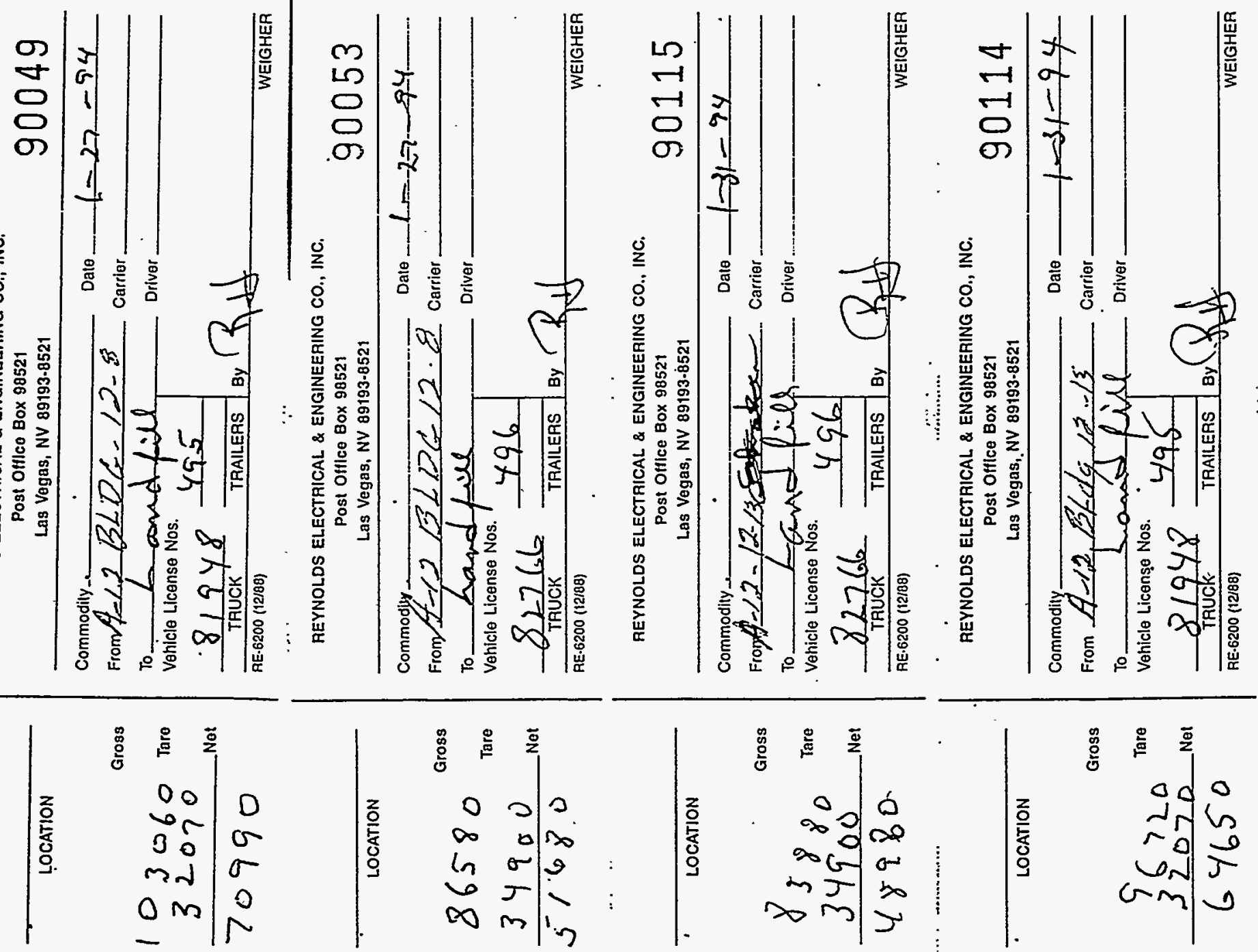
1. TPackage will include the following items:

\begin{tabular}{|c|c|c|}
\hline & \multicolumn{2}{|c|}{ UST 12-13-1 REM } \\
\hline & YES & NO \\
\hline b. Characteristation letter & $x$ & \\
\hline c. Analytical Results & $x$ & \\
\hline d. Process Knowledge & & $x$ \\
\hline e. Is the package complete? & $\bar{x}^{2}$ & \\
\hline f. Basic Description & $\approx 7$ & \\
\hline
\end{tabular}

\begin{tabular}{|c|c|}
\hline $\begin{array}{c}\text { Brady \# } \\
\text { YES }\end{array}$ & NO \\
\hline & \\
\hline & \\
\hline & \\
\hline & \\
\hline
\end{tabular}

\begin{tabular}{|c|c|}
\hline $\begin{array}{c}\text { Brady \# } \\
\text { YES }\end{array}$ \\
\hline & NO \\
\hline & \\
\hline & \\
\hline & \\
\hline
\end{tabular}

\begin{tabular}{|c|c|}
\hline \begin{tabular}{|l|}
\hline \multicolumn{2}{|c|}{$\begin{array}{l}\text { Nrady \# } \\
\text { YES }\end{array}$} \\
\hline
\end{tabular} \\
\hline & \\
\hline & \\
\hline & \\
\hline & \\
\hline
\end{tabular}

2. SCHEDULE A DATE AND TIME FOR DISPOSAL
a. Date Scheduled:
MAY 9, 1995
C. Load Number
b. Brady Numbers:

\begin{tabular}{l}
$592 \cdot 360$ \\
Start Date: NAMI 9, 1995 \\
\hline
\end{tabular}
End Date: NAY 9,1995

3. AT THE LANDFILL, THE GENERATOR MUST HAVE:

\begin{tabular}{|l|l|l|}
\cline { 2 - 3 } \multicolumn{1}{c|}{} & YES & NO \\
\hline a. Bill of landing \# $\&$ & & \\
\hline b. Rad Sticker & & \\
\hline c. Weight ticket & & \\
\hline d. Work Order Number & & \\
\hline
\end{tabular}

WEIGHT TOTAL
WORK ORDER \#
$3754-0.38$

\begin{tabular}{l}
\hline $43,020165$. \\
SUB/ACCT. FREQ. \\
\hline
\end{tabular}

\section{COMPLETE PACKAGE}

\begin{tabular}{|l|c|c|}
\cline { 2 - 3 } \multicolumn{1}{c|}{} & YES & NO \\
\hline a. Weight "entered into database & & \\
\hline b. Enter weight for monthly ATV & & \\
\hline c. Filed in sequence & & \\
\hline d. File in Storage Cabinet & & \\
\hline
\end{tabular}

Signature/D ate:

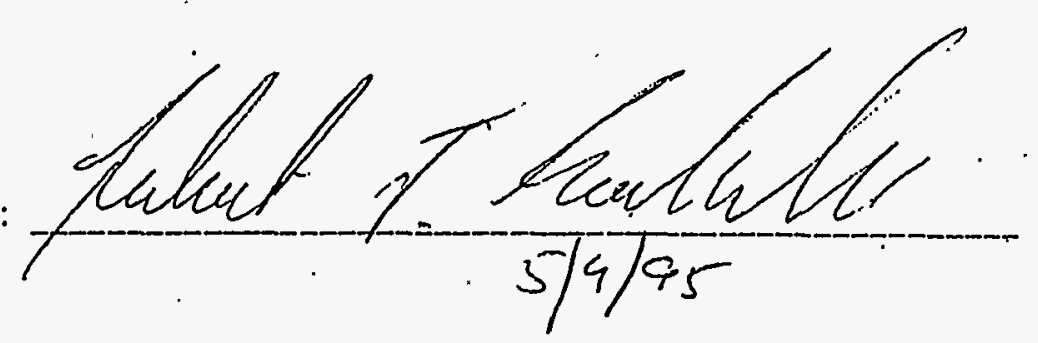


TPackage will include the following items:

\begin{tabular}{|l|c|c|}
\cline { 2 - 3 } \multicolumn{1}{c|}{} & \multicolumn{3}{c|}{$\begin{array}{c}\text { Brady } \# 592-212 \\
\text { YES }\end{array}$} \\
\hline b. Characteristation letter & $X$ & NO \\
\hline c. Analytical Results & $\not$ & \\
\hline d. Process Knowledge & & $X$ \\
\hline e. Is the package complete? & \multicolumn{1}{|c|}{. } & \\
\hline f. Basic Description & 245 C.Y. \\
\hline
\end{tabular}

\begin{tabular}{|c|c|}
\hline $\begin{array}{c}\text { Brady \# } \\
\text { YES }\end{array}$ & NO \\
\hline & \\
\hline & \\
\hline & \\
\hline & \\
\hline
\end{tabular}

\begin{tabular}{|c|c|}
\hline $\begin{array}{c}\text { Brady \# } \\
\text { YES }\end{array}$ & NO \\
\hline & \\
\hline & \\
\hline & \\
\hline & \\
\hline
\end{tabular}

\begin{tabular}{|c|c|}
\hline $\begin{array}{c}\text { Brady \# } \\
\text { YES }\end{array}$ & No \\
\hline & \\
\hline & \\
\hline & \\
\hline & \\
\hline
\end{tabular}

SCHEDULE A DATE AND TIME FOR DISPOSAL
a. Date Scheduled:
MAN 9, 1992
C. Load Number
b. Brady Numbers:
$592-212$
Start Date:
End Date:

AT THE LANDFILL, THE GENERATOR MUST HAVE:

\begin{tabular}{|c|c|c|c|c|c|}
\hline & YES & NO & & & \\
\hline a. Bill of landing $\Psi 1-5$ & 7 & & WEIGHT TOTAL & 240 & $740 \mathrm{lbs}$ \\
\hline b. Rad Sticker & 7 & & \multirow{3}{*}{$\begin{array}{l}\text { WORK ORDER \# } \\
1090-143\end{array}$} & \multirow{3}{*}{ SUB/ACCT. } & \\
\hline c. Weight ticket & 7 & & & & FREQ. \\
\hline d. Work Order Number & 7 & & & & \\
\hline
\end{tabular}

COMPLETE PACKAGE

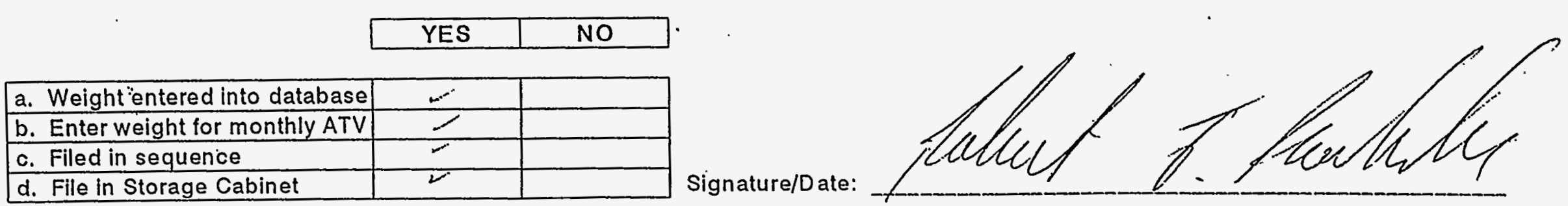




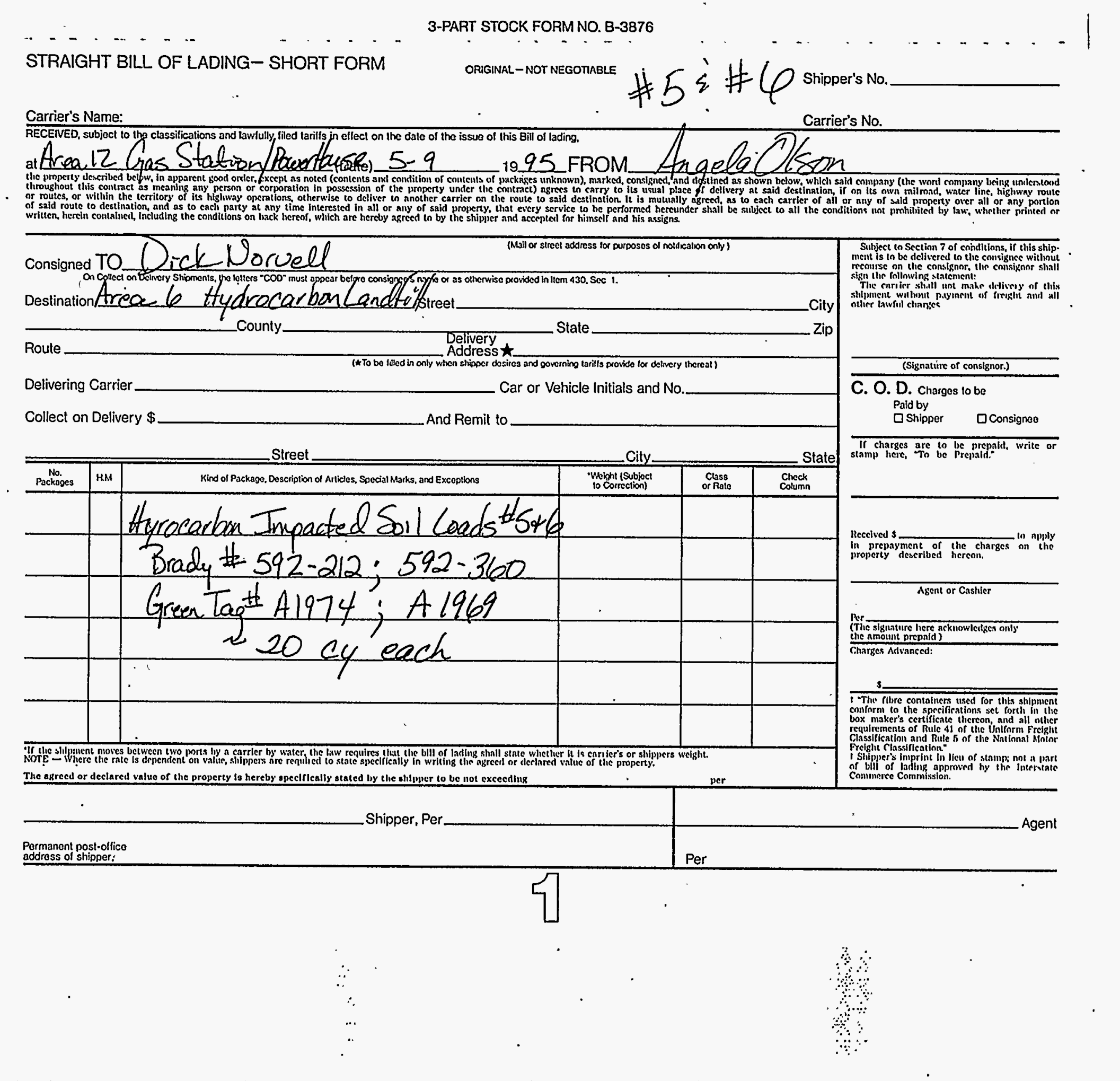




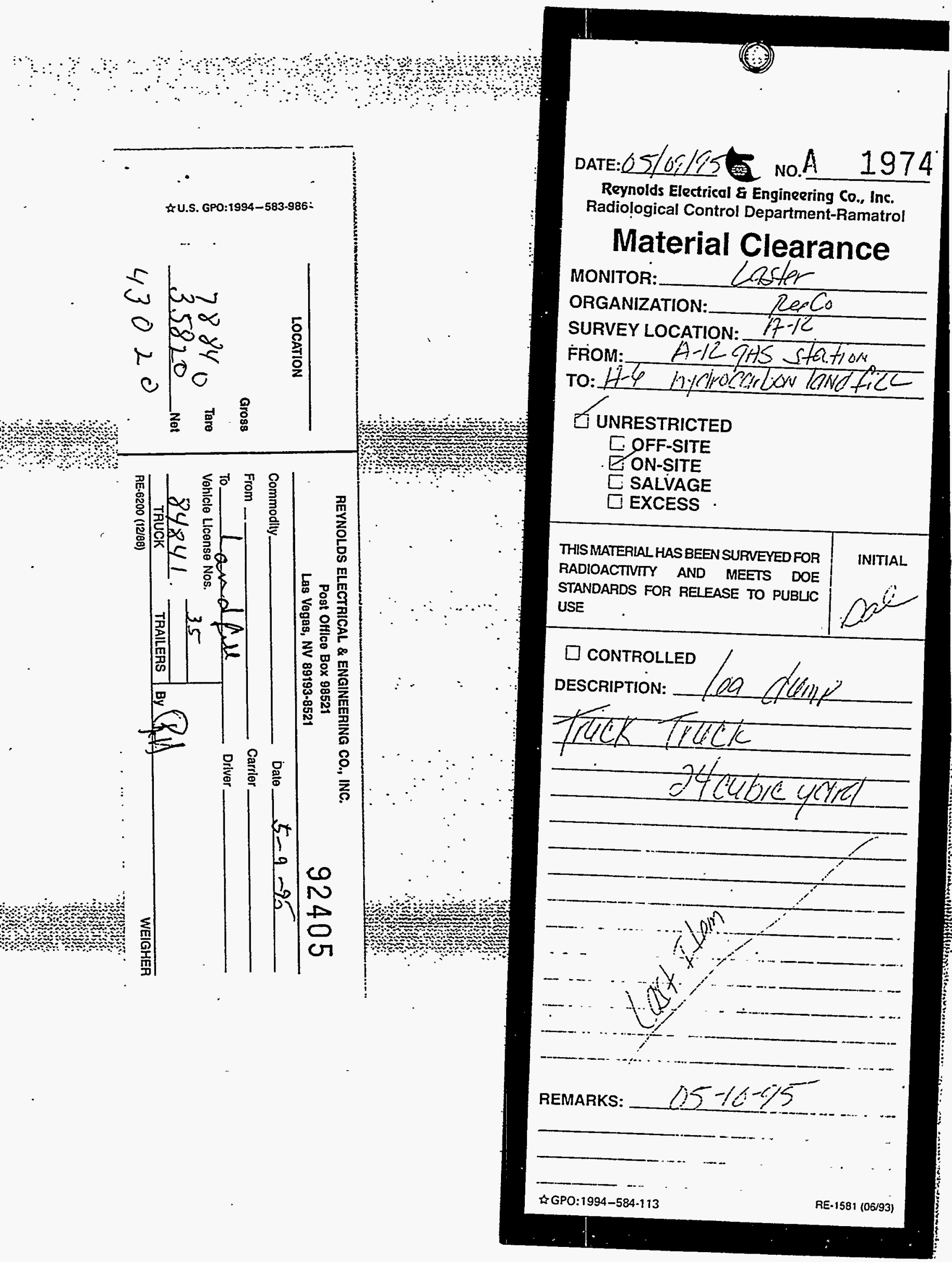




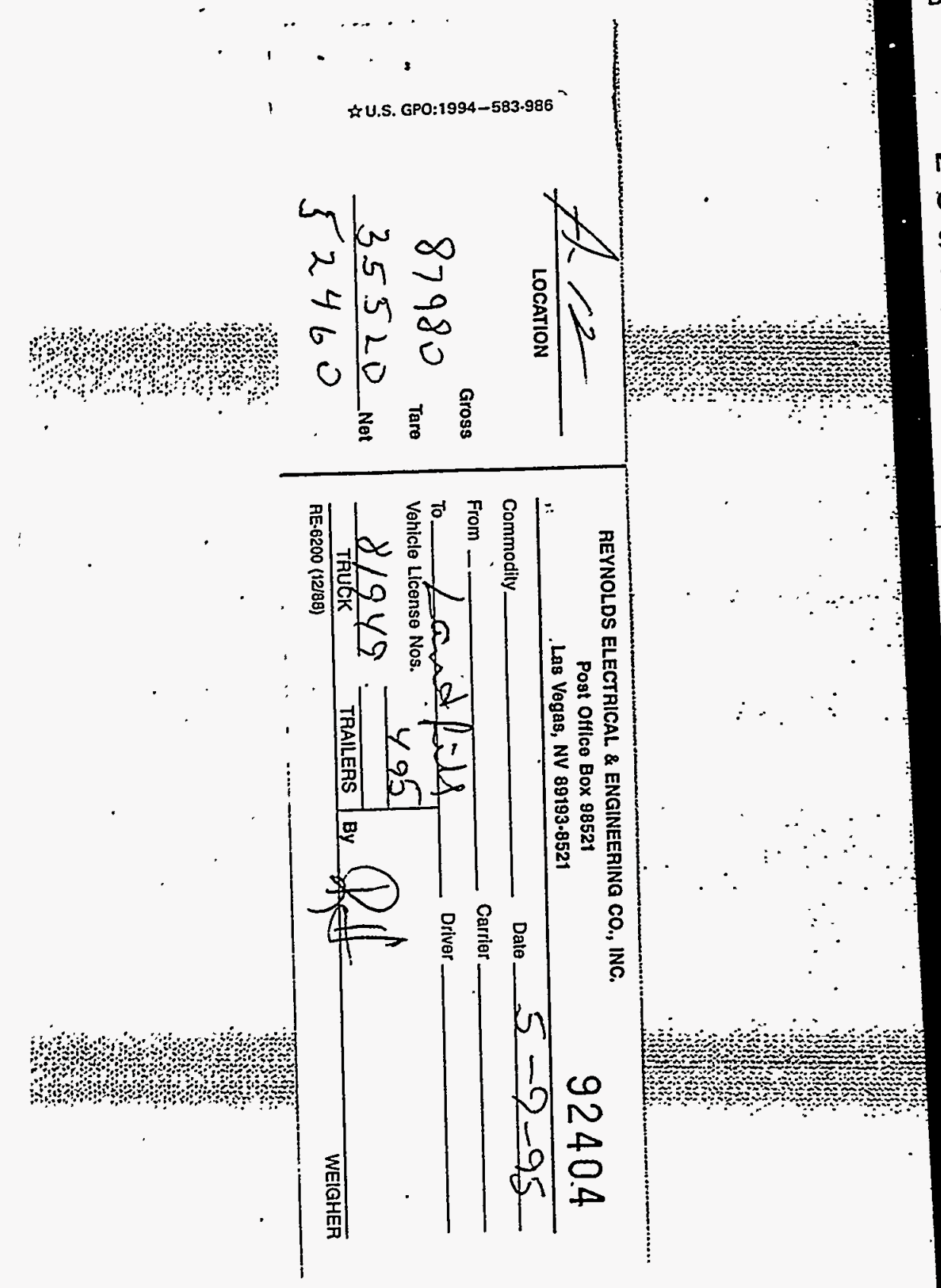

$\because$
DATE:
Reynolds Electrical a Engineering Co.. Inc. Rediological Control Department-Ramatrol

Material Clearance MONITOR:

ORGANIZATION:

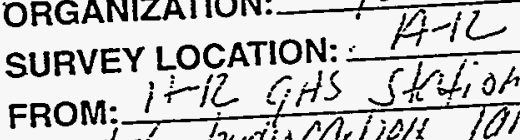

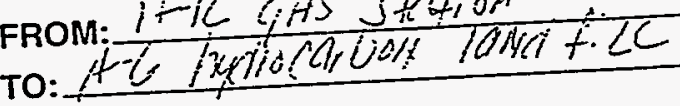

\section{UNRESTRICTED \\ $\square$ OFF-SITE \\ ON-SITE \\ $\square$ SALVAGE \\ $\square$ EXCESS}

THIS MATERLAL HAS BEEN SURVEYED FOR RADIOACTIVITY AND MEETS DOE STANDARDS FOR RELEASE TO PUBLIC USE

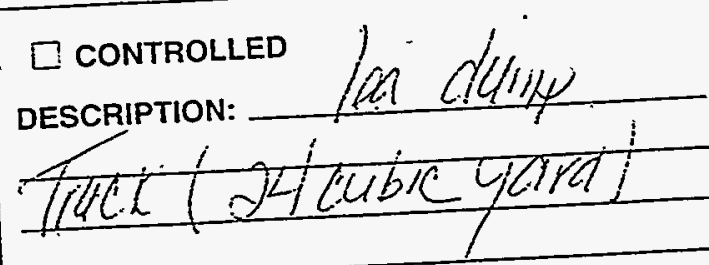

REMARKS:
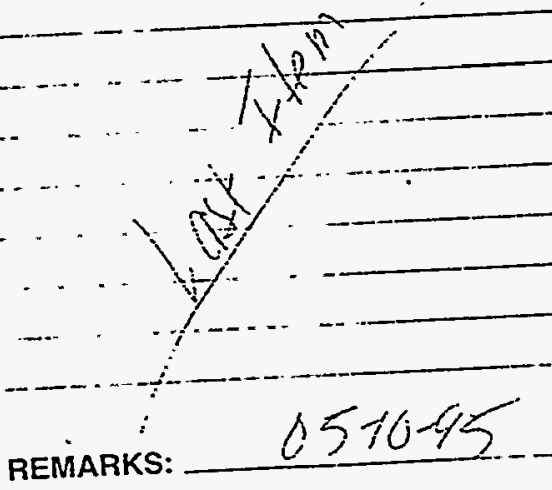
ORIGINAL-NOT NEGOTABLE

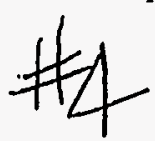

Carrier's Name: AECEIVED, subfect to the classificalions and lawlully filed larills in effect on tho dato of the issue of this Bill of lading at Area 12 Gas Station (Date) $5-9$ 19. 25 FROM

Shipper's No.

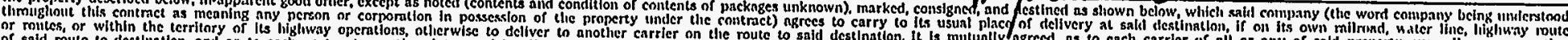

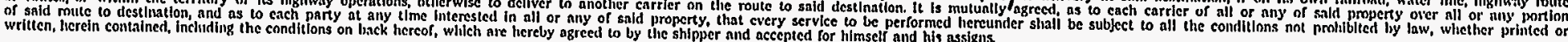

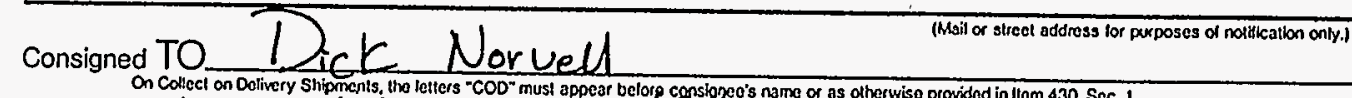
Destination Axeaufe. Hydrocarbon Land fill street.

\section{Route} County

Delivering Carrier Delivery

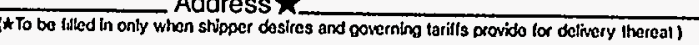
Car or Vehicle Initials and No.

Collect on Delivery $\$$ And Remit to

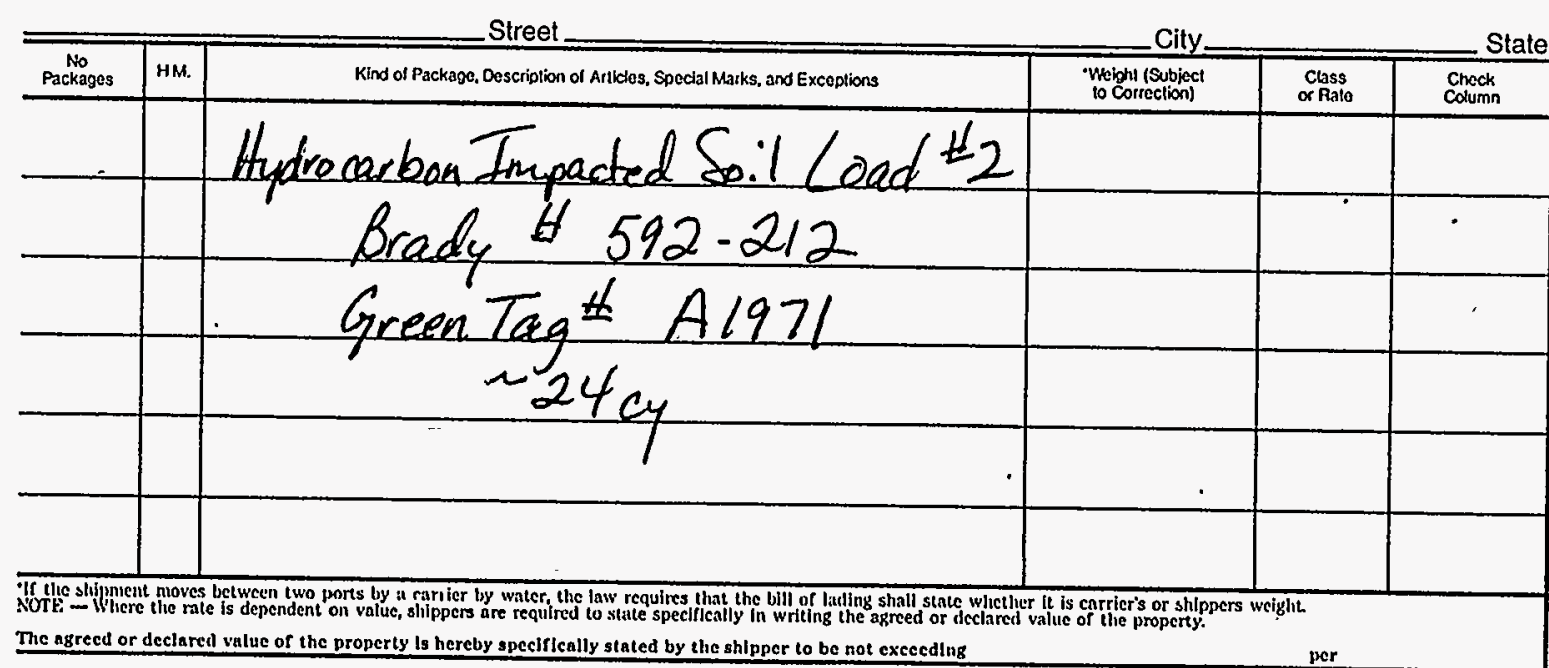
The agrecd or declared value of the property is herelby specifleally stated by the shlpper to be not exceeding and $\frac{\square \text { Shipper } \quad \square \text { Consignee }}{\text { If clarges are to pee preppitil, write or }}$

mats

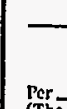
Per
(The slgiature hiere acknowledges only
the amount prepand.)
Cliarges Aelvanced:

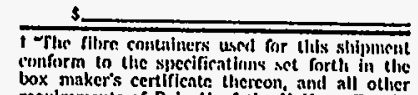

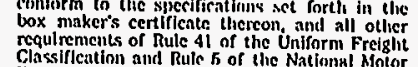

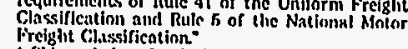

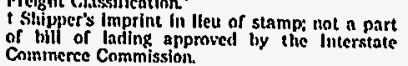
Shipper, Per

Permanent post-office
address of shipper Ther

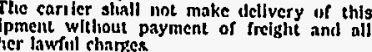
(Signature of consignur.)

D. Changes to be

Paid by (a)

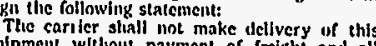
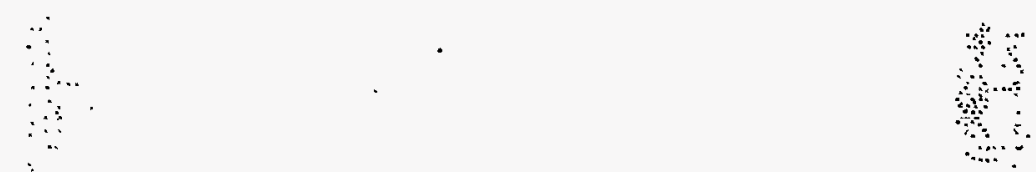


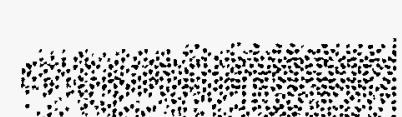

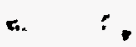

น U.S. GPO:1994-583-986
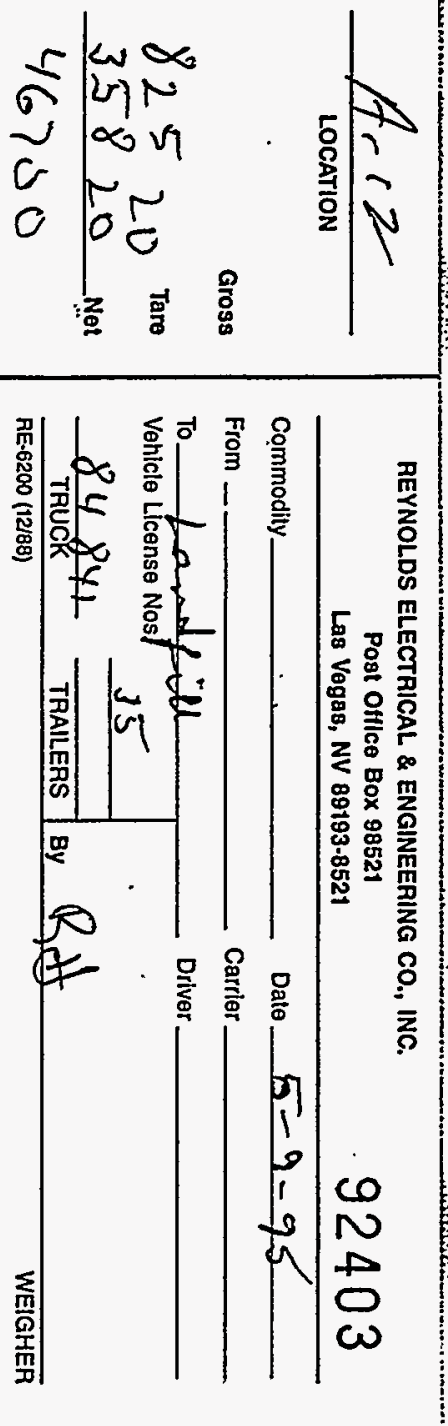

DATE: 65 lo9/95 No.A 1972

Reynolds Electrical \& Engineering Co., Inc.

- Radiological Control Department-Ramatrol

Material Clearance MONITOR:

ORGANIZATION: RleCO

SURVEY LOCATION: $12-12$

FROM: A $A-i 2$ OHE Station

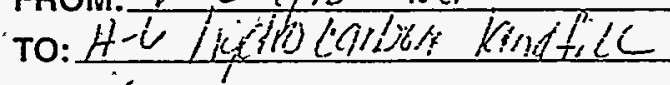

CUNRESTRICTED

$$
\begin{aligned}
& \text { OFF-SITE } \\
& \text { ON-SITE } \\
& \text { SALVAGE } \\
& \text { EXCESS }
\end{aligned}
$$

THIS MATERLAL HAS BEEN SURVEYED FOR RADIOACTIVTY AND MEETS DOE STANDARDS FOR RELEASE TO PUBLIC

USE

INITIAL

E CONTROLLED DESCRIPTION:

\section{Ion dunip Truck}
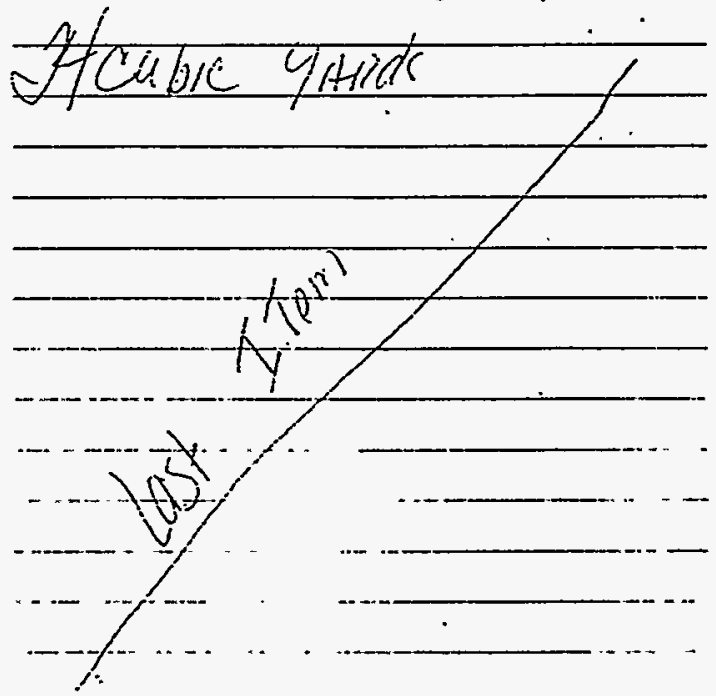

REMARKS:

$0,5-18-45$ 
Carrier's Name: Carrier's No.

RECEIVED. subjoct to the classillcations and lawfully filed tarills in elfoct on the date of the Issue of this Bill of lading. at Area 12 cas Staftion (Date) 5-9

25 FROM

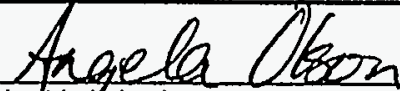

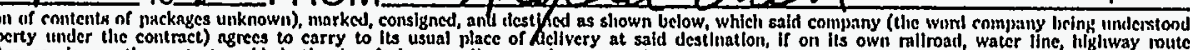

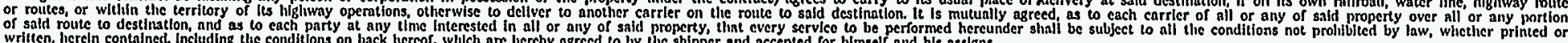

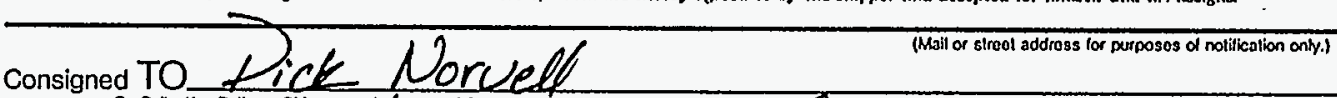

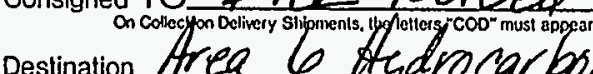

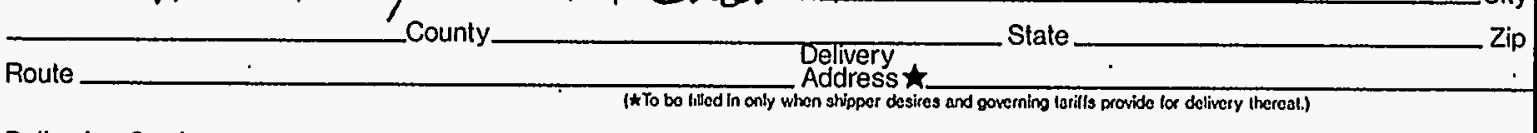

Delivering Carrier Car or Vehicle Initials and No.

Collect on Delivery $\$$ And Remit to

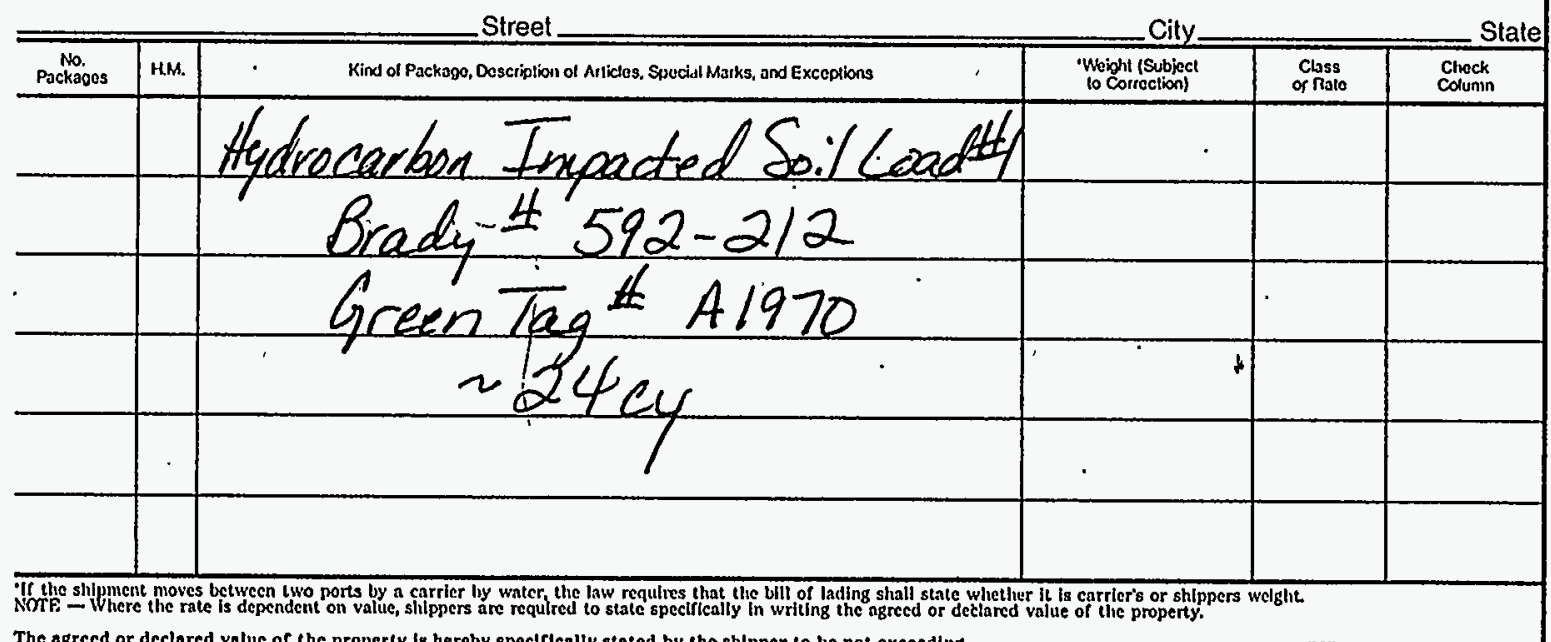

Subiject 10 Section 7 of conditions, If this ship the conslgnor stial

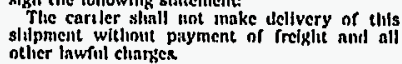

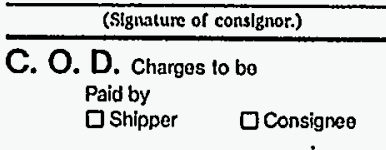
If charges are to be prepard, white or
stamp liere, To be Prepald,:

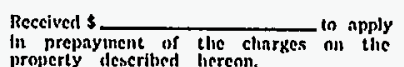
Agent or Cashilier Per
The signature hore acknowledges onls
the amount prepald.). Clarges Alvanced:

The fitre contalners used for thils slipment
conform to thic specifications sel fortli iti the box maker's certlficate clicreon, and all other

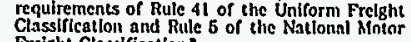

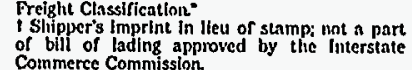
The agreed or declared value of the property is hereby specifically stated by the shlpper to be not exceeding

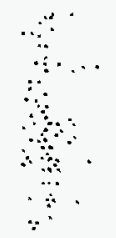




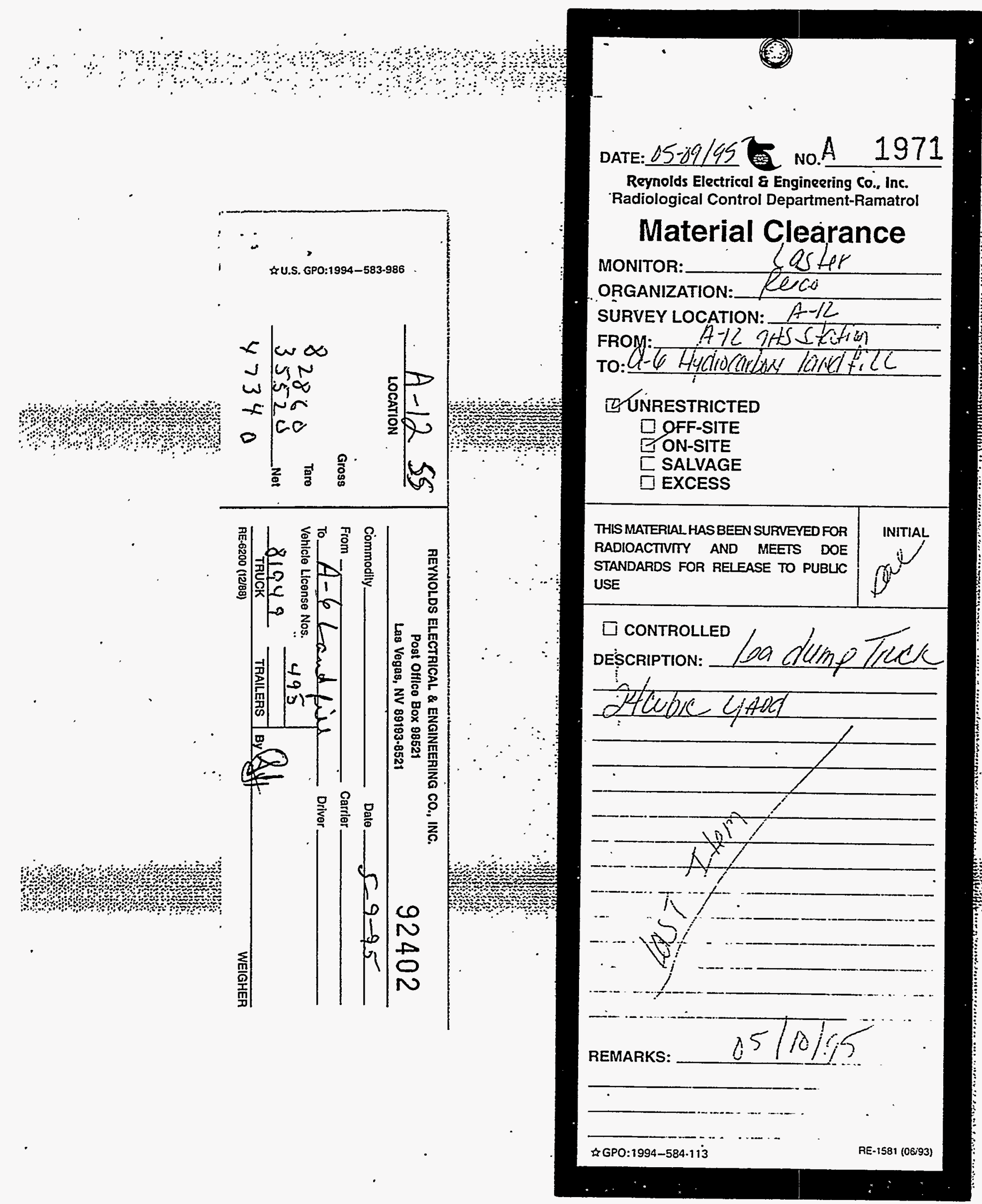


1... _. . . . . . . . . . .

STRAIGHT BILL OF LADING-SHORT FORM

Carrier's Name:

RECEIVED, subjecl to the classificalions and lawlully filed tarills in eflect on the date of the issue of this Bill of lading.

at Area 12 Gas Station

(Date) $5-9$

19.95 FROM

Carrier's No.

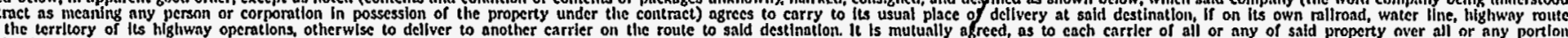

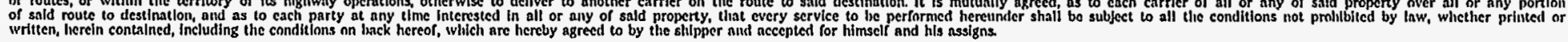

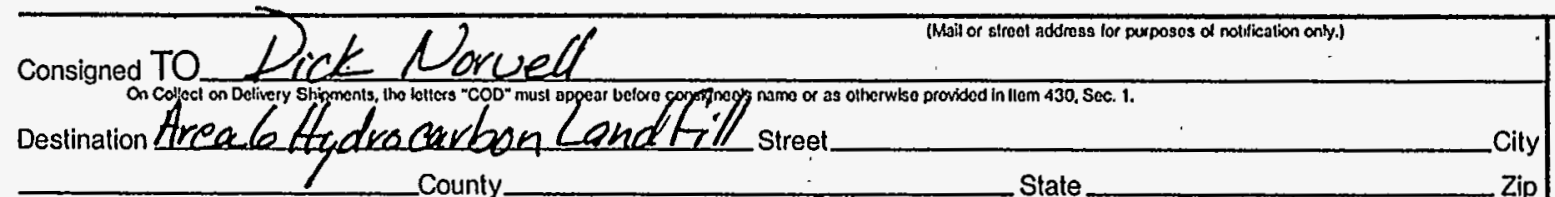

Route_C Delivery

Delivering Carrier

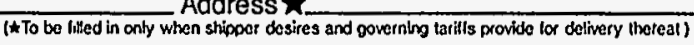
Car or Vehicle Initials and No.

Collect on Delivery $\$$ And Remit to

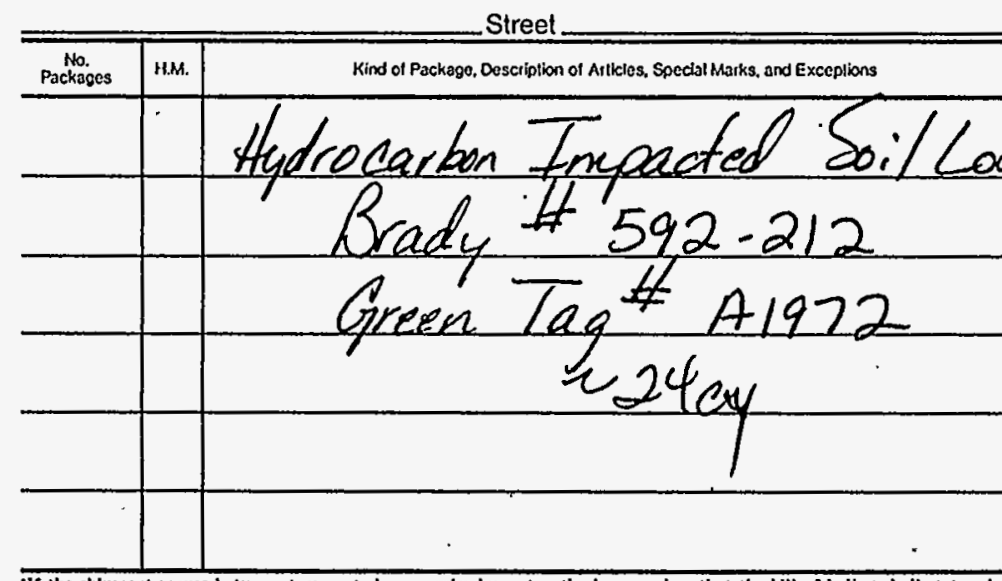

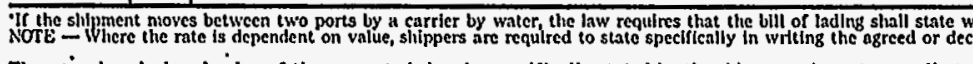

The agreed or declared value of the property is hereby speciffleally stated by the shipper to be not exceedlag

Shipper, Per

Permanent post-olfice
address ol shipper,

\begin{tabular}{|c|c|c|}
\hline $\begin{array}{l}\text { Whibht (Subioct } \\
\text { to Corroction) }\end{array}$ & $\begin{array}{l}\text { Class } \\
\text { of Rale }\end{array}$ & $\begin{array}{l}\text { Check } \\
\text { Column }\end{array}$ \\
\hline & & \\
\hline
\end{tabular}

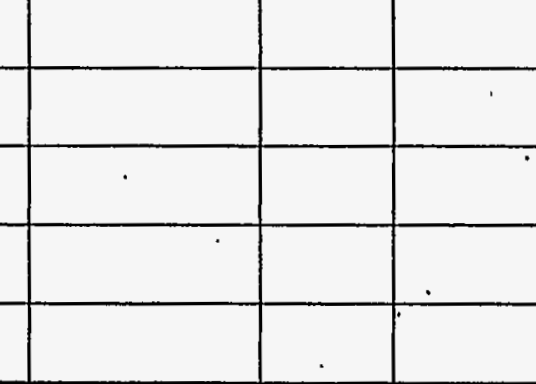

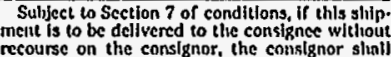

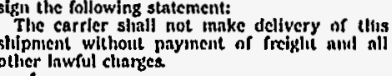

$\frac{\text { (Signature nf consignor.) }}{\text { C. } O . \text { D. Charges to be }}$

Paid by

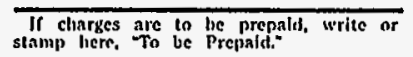

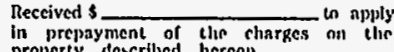

Agent m Cishiter

Per
(The signature here acknowledges only
the amoun Ctanges Aelvaniced:

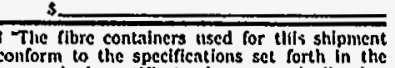

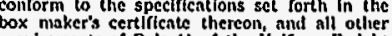

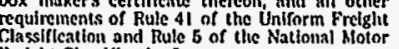
recight Classificallon" -

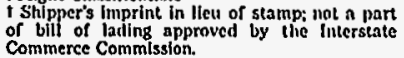




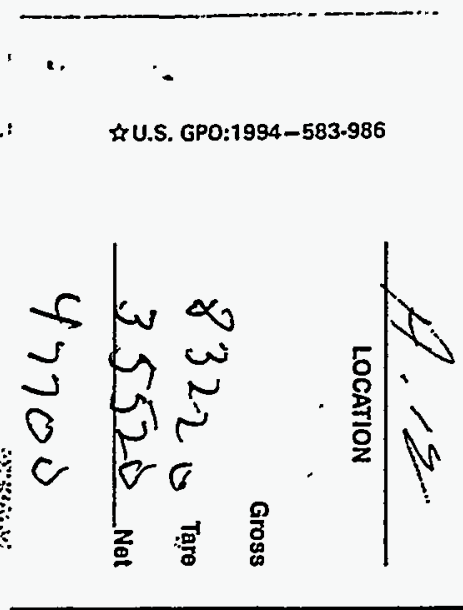

DATE:05/OI/45 No.A 197.0

Reynolds Electrical \& Engineering Co., Inc. Radiological Control Department-Ramatrol

Material Clearance MONITOR:

ORGANIZATION:

aliv

SURVEY LOCATION: $A-12$

FROM: 12 aths statiol

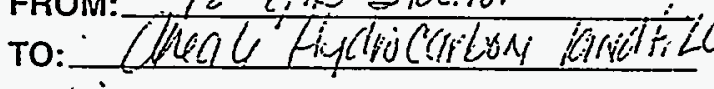

QÚNRESTRICTED
$\square$ GFF-SITE
QON-SITE

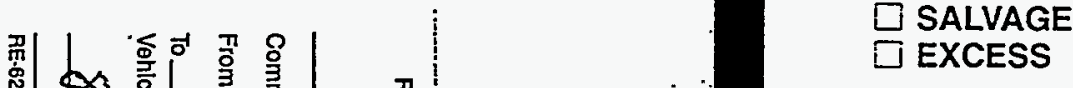

THIS MATERLAL HASBEENSURVEYED FOR RADIOACTIVTYY AND MEETS DOE STANDARDS FOR RELEASE TO PUBLIC USE

INITIAL

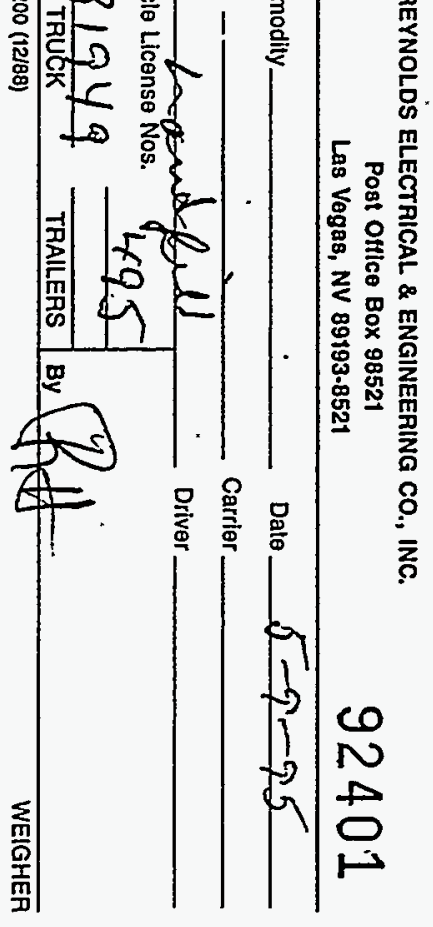

Ð CONTROLLED

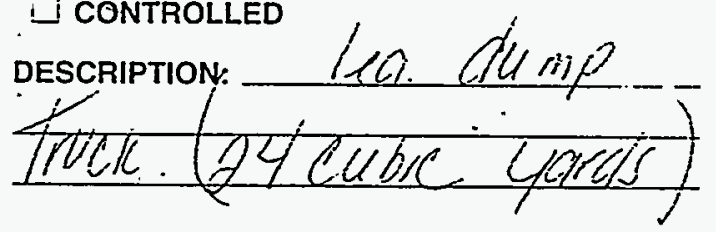

REMARKS:

$05-10 / 95$ 
Carrier's Name:

ORIGINAL-NOT NEGOTIABIE

Shipper's No.

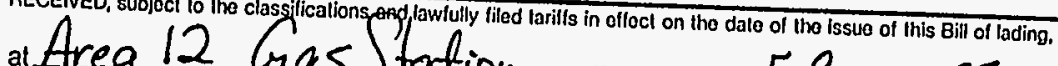

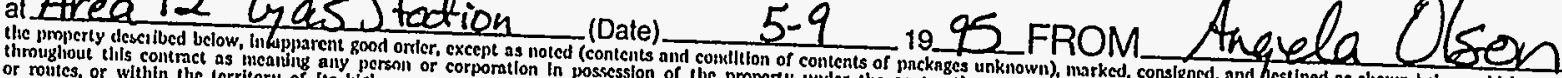

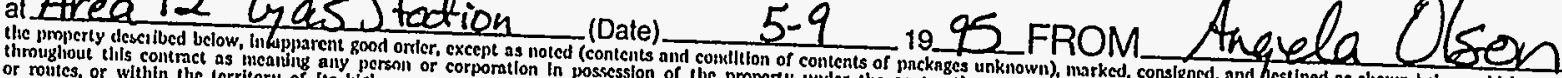
at Area 12 Gas tation

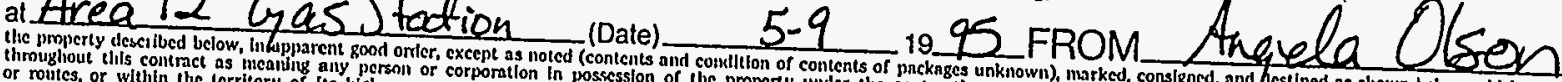
Carrier's No

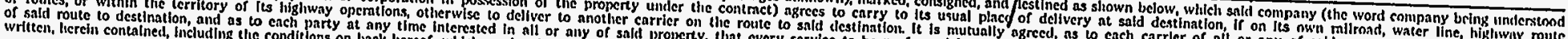

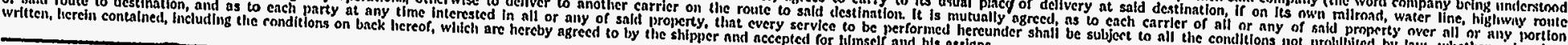
Consigned TO lick Nor ve il

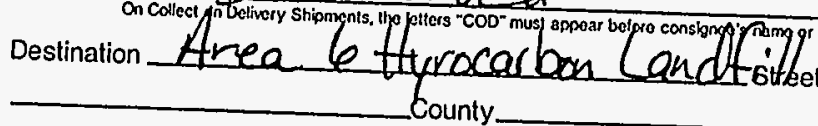
(Mail or stroel address lor purposes of nollication onty.)

Route

Delivering Carrier ATo bo lithed in orly Address

$$
\text { Delivery }
$$
dosires and governing willts provide for delivery therceat) Car or Vehicle Initials and No. And Remit to
Collect on Delivery $\$$
City_________ate

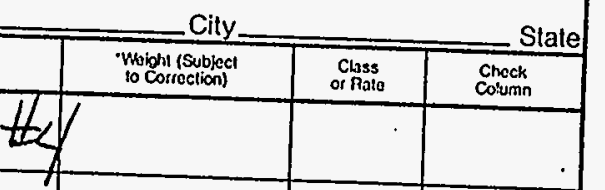

\section{- Received s} in prepayment of the charges to apply
property described thereon.

\begin{tabular}{|c|}
\hline Agent or Cashier \\
\hline 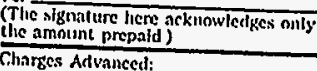 \\
\hline
\end{tabular}

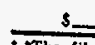

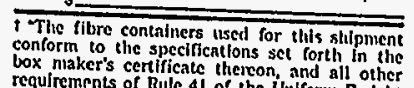

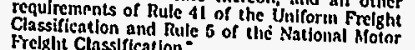

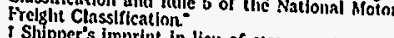

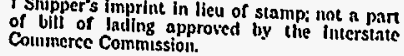

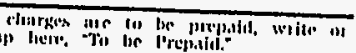

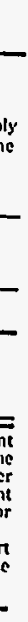

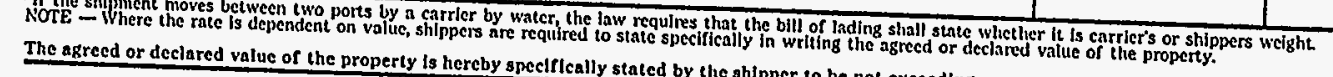

D. Charges to be PShipper aconsignee Per 


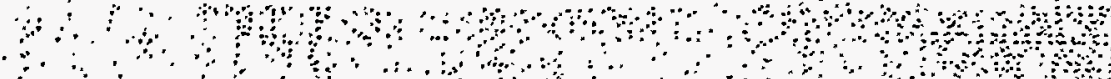

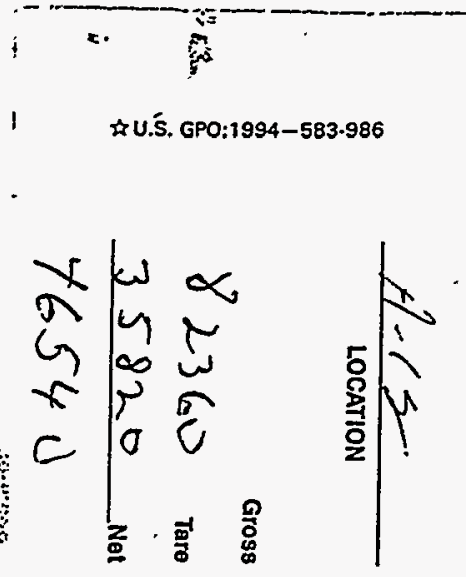

DATE: $85 / 69 / 95$ No.A 1969

Reynolds Electrical \& Engineering Co., Inc. Radiological Control Department-Ramatrol

Material Clearance MONITOR:_LQLLE!

ORGANIZATION: ReECO

SURVEY LOCATION: $\quad 17-12$ FROM: GAS sfation/power lizks

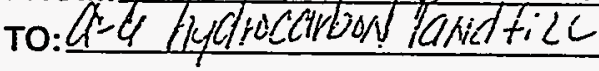

\section{GUNRESTRICTED

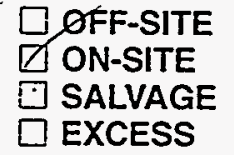

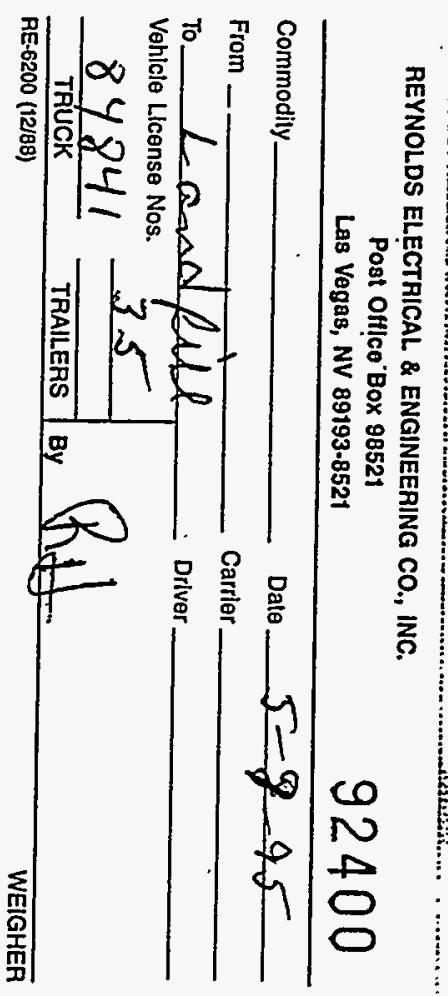

THIS MATERLAL HAS BEEN SURVEYED FOR

RADIOACTIVITY AND MEETS DOE STANDARDS FOR RELEASE TO PUBLIC USE

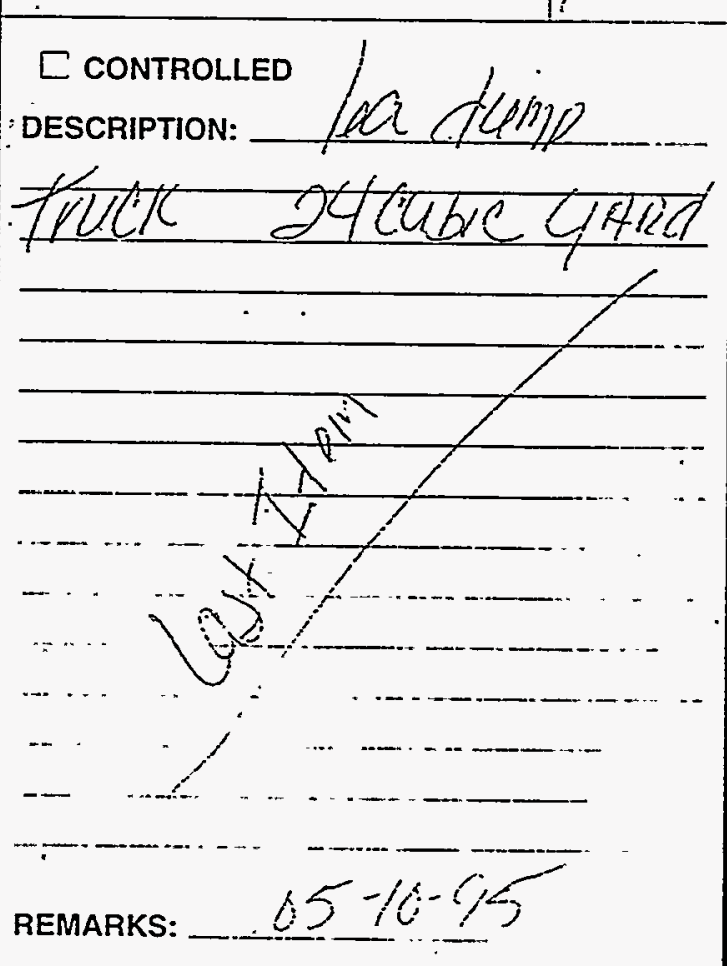




\section{Copies}

U. S. Department of Energy, Nevada Operations Office

P. O. Box 98518

Las Vegas, NV 89193-8518

J. Appenzeller-Wing

S. T. Curtis

Public Reading Room

Technical Information Resource Center

U.S. Department of Energy

Office of Scientific and Technical Information

175 Oak Ridge Turnpike

P.O. Box 62

Oak Ridge, Tennessee 37831

Bechtel Nevada

P. O. Box 98521

Las Vegas, NV 89193-8521

J. F. Bonn

J. R. Kannard

D. D. Madsen

S. J. Nacht

M. D. Shotton

S. R. Weil 